\title{
Evaluating medication utilization patterns and healthcare outcomes in patients receiving antipsychotics
}

\author{
Mariam K. Hassan \\ West Virginia University
}

Follow this and additional works at: https://researchrepository.wvu.edu/etd

\section{Recommended Citation}

Hassan, Mariam K., "Evaluating medication utilization patterns and healthcare outcomes in patients receiving antipsychotics" (2005). Graduate Theses, Dissertations, and Problem Reports. 2283.

https://researchrepository.wvu.edu/etd/2283

This Dissertation is protected by copyright and/or related rights. It has been brought to you by the The Research Repository @ WVU with permission from the rights-holder(s). You are free to use this Dissertation in any way that is permitted by the copyright and related rights legislation that applies to your use. For other uses you must obtain permission from the rights-holder(s) directly, unless additional rights are indicated by a Creative Commons license in the record and/ or on the work itself. This Dissertation has been accepted for inclusion in WVU Graduate Theses, Dissertations, and Problem Reports collection by an authorized administrator of The Research Repository @ WVU.

For more information, please contact researchrepository@mail.wvu.edu. 


\title{
Evaluating Medication Utilization Patterns and Healthcare Outcomes in Patients receiving Antipsychotics
}

\author{
Mariam K. Hassan \\ Dissertation submitted to the \\ School of Pharmacy \\ at West Virginia University \\ in partial fulfillment of the requirements \\ for the degree of \\ Doctor of Philosophy \\ in \\ Pharmaceutical Systems and Policy \\ S. Suresh Madhavan, M.B.A., Ph.D., Chair \\ Syed Islam, Ph.D. \\ Iftekhar Kalsekar, Ph.D. \\ Jan Kavookjian, M.B.A., Ph.D. \\ Eugene Makela, Pharm.D. \\ Lesley-Ann Miller, Ph.D.
}

Department of Pharmaceutical Systems and Policy

\section{Morgantown, West Virginia \\ 2005}

Keywords: Antipsychotic, Schizophrenia, Bipolar Disorder, Medicaid, Adherence, Medication Utilization Pattern, Healthcare Utilization, Costs

Copyright 2005 Mariam Hassan 


\title{
ABSTRACT \\ Evaluating Medication Utilization Patterns and Healthcare Outcomes in Patients receiving Antipsychotics
}

\author{
Mariam K. Hassan
}

Several atypical antipsychotics have entered the market since the last decade. Evaluating the utilization patterns and overall cost savings generated by these expensive agents is becoming more important as their use continues to expand. Phase 1 of this study describes the patterns of antipsychotic utilization and its impact on total and mental health-related costs for schizophrenia and bipolar disorder patients in a state Medicaid program. Phase 2 of this study compares typical antipsychotics, risperidone, olanzapine, and quetiapine in terms of direct costs and utilization of healthcare services such as hospitalizations, emergency room visits, outpatient visits, psychiatric prescription use and antipsychotic therapy modifications among schizophrenia and bipolar disorder patients. A retrospective, longitudinal study design was employed and a state Medicaid claims data from January 1, 1998 to December 31, 2002 was used. Multivariate analysis was used to statistically control for various confounding factors including patient demographics, prescribing physician type, mental health diagnosis, other comorbidities, pre-index alcohol and substance abuse, pre-index concomitant medication use, and preindex healthcare utilization. Phase 1 results revealed that a large proportion of schizophrenia and bipolar disorder patients are non-adherent to antipsychotic therapy. Schizophrenia and bipolar disorder patients who are non-adherent to antipsychotic therapy or receive antipsychotic polytherapy incurred significantly higher total and mental healthcare costs. Phase 2 results revealed that there were no significant differences in total and mental healthcare costs among schizophrenia patients initiated on any of the study antipsychotics. Bipolar disorder patients initiated on typical antipsychotics incurred higher total and mental healthcare costs as compared to patients initiated on atypicals. There were no significant differences in total and mental healthcare costs among patients initiated on any of the study atypical antipsychotics. Patients initiated on olanzapine incurred highest pharmacy costs whereas patients initiated on typical antipsychotics incurred lowest pharmacy costs. Schizophrenia and bipolar disorder patients initiated on typical antipsychotics showed significantly lower adherence and higher likelihood of modifying antipsychotic therapy as compared to atypicals. 


\section{ACKNOWLEDGEMENTS}

First and foremost, I would like to thank Dr. Suresh Madhavan for bringing me into the research program at WVU and giving me the opportunity to learn and develop my skills in the warm, friendly and stimulating environment of our PSP department. As my dissertation chairperson, his advice, assistance and encouragement were crucial in the development and completion of this dissertation project.

I would like to thank Dr. Syed Islam for the guidance, support and invaluable learning experience I received while working with him. His insight and suggestions were very helpful throughout the course of this study. I sincerely appreciate the time and help of my committee members - Dr. Iftekhar Kalsekar, Dr. Jan Kavookjian, Dr. Eugene Makela and Dr. Lesley-Ann Miller. Their expertise and advice were essential in improving this project. I would like to thank Dr. Kitty Rajagopalan and AstraZeneca for giving the opportunity and funding support to carry out this project. I would also like to acknowledge Ms. Peggy King, Pharmacy Program Coordinator, West Virginia Bureau of Medical Services, for her support and approval to conduct this study. I want to thank Dr. Mike Smith for his informative inputs about the database use and Dr. Virginia Scott for her advice and support all through my graduate school years. I thank Kelly White and Mickey Howell for being very cooperative and helpful at all times. I also appreciate Rahul's assistance with the tables for this study.

I will always cherish the wonderful friends I made during my school years. They have filled the long years of my doctoral studies with fun, laughter and memorable moments. I would specially like to mention Vivek, Murali and Anna from the department for always being there to help, support or just hang out. 
The greatest credit for this work goes to my parents for all for the attention and care they have put into my education from early on. The love, patience and encouragement from all my family members- Abba, Mummy, Nazia, Aamer and Akash has given me the strength to get to this point. The lengthy and trying phases of this work would have been difficult to overcome without the reassurance and motivation from Akash. I will always be indebted to my loving family for everything I achieve in life. 


\section{TABLE OF CONTENTS}

ABSTRACT

ACKNOWLEDGEMENTS .....................................................................................ii

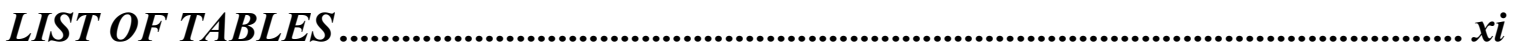

LIST OF FIGURES.........................................................................................

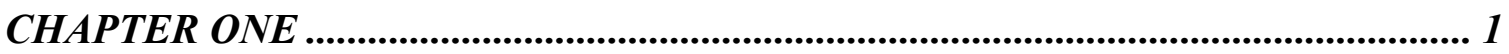

INTRODUCTION.............................................................................................................. 1

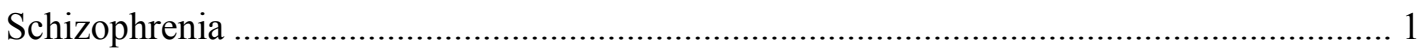

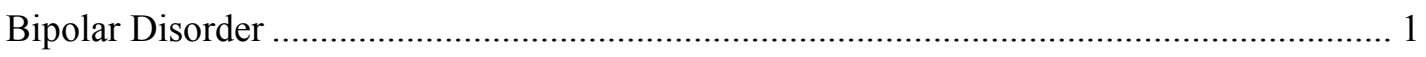

Antipsychotic Treatment Patterns ................................................................................ 2

Antipsychotic Therapy and Economic Outcomes............................................................ 5

STATEMENT OF PROBLEM.................................................................................... 7

CONCEPTUAL FRAMEWORK.............................................................................. 9

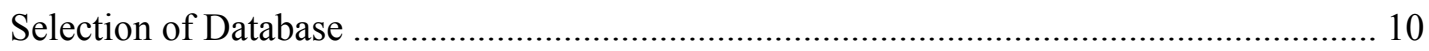

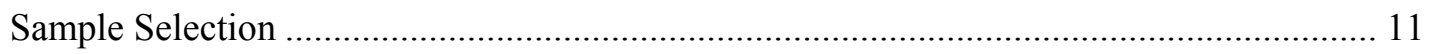

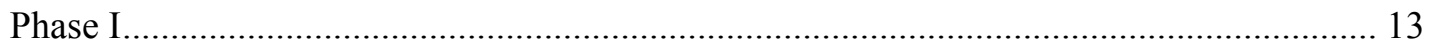

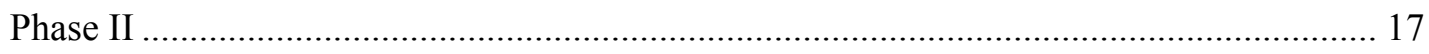

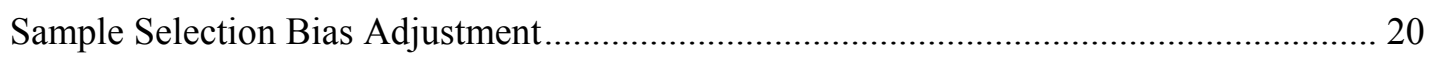

STUDY OBJECTIVES.............................................................................................. 22

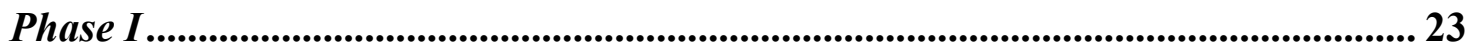

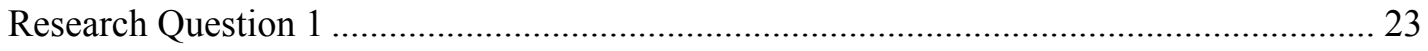

Research Question 2 .......................................................................................... 23

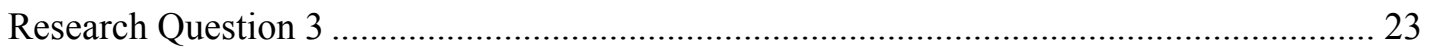

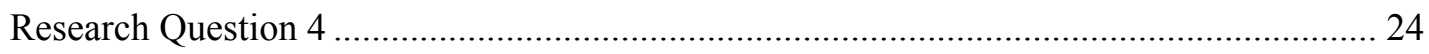




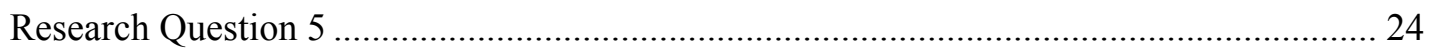

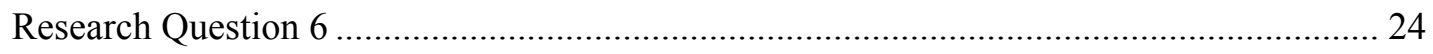

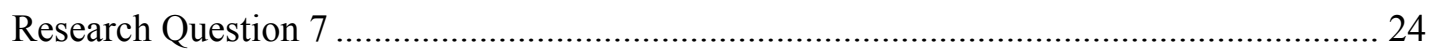

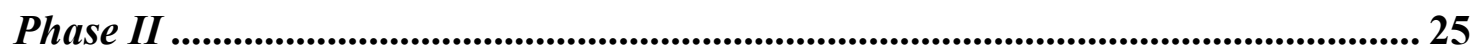

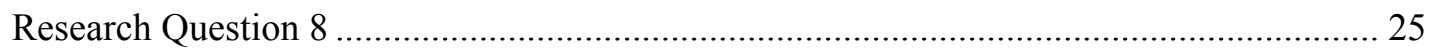

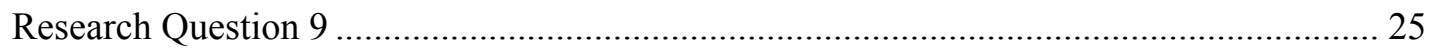

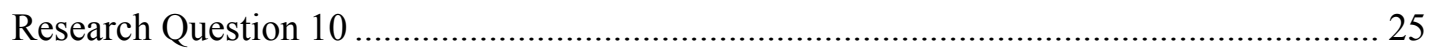

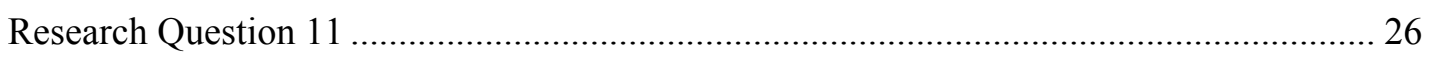

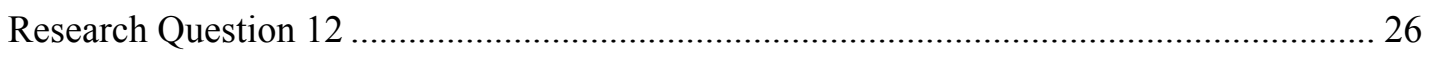

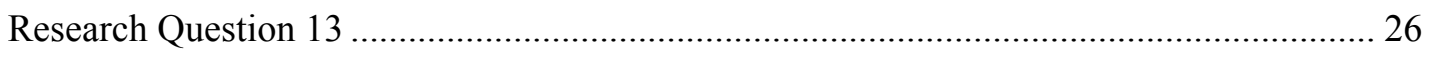

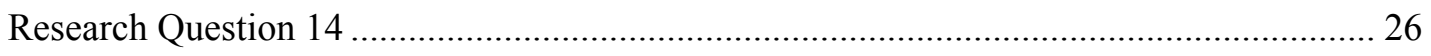

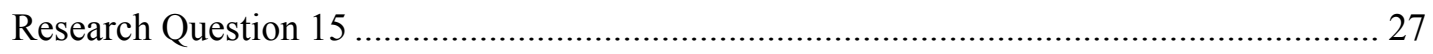

STUDY SIGNIFICANCE _........................................................................................... 27

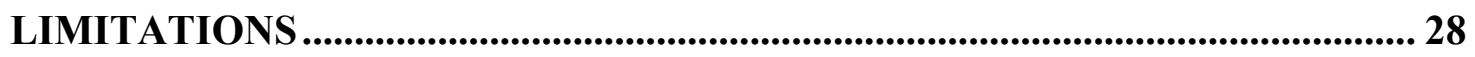

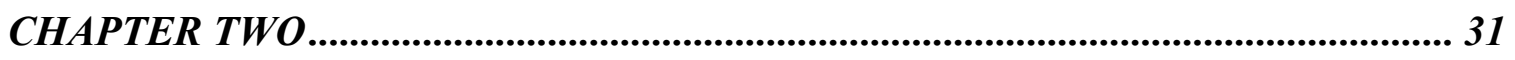

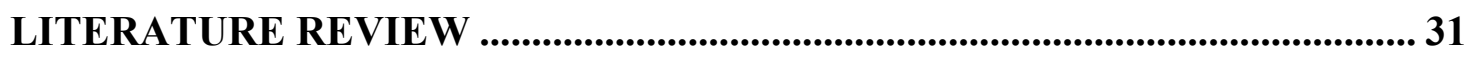

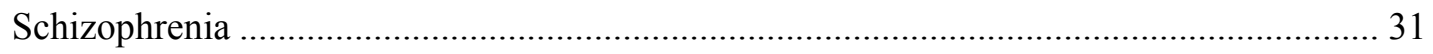

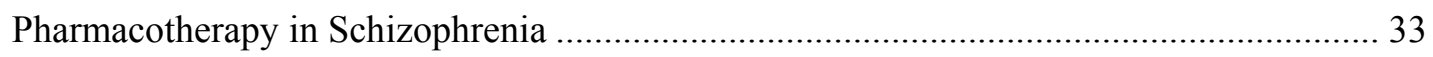

Antipsychotic Treatment Guidelines for Schizophrenia .................................................. 36

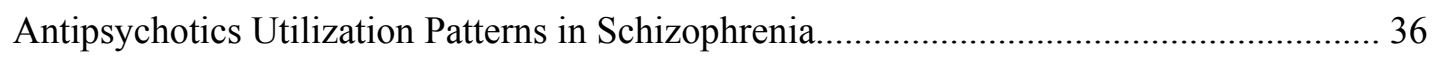

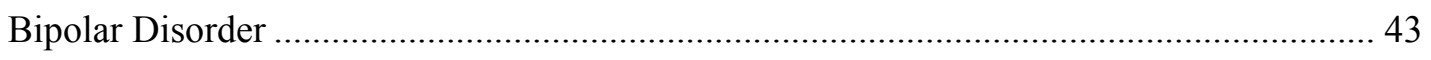

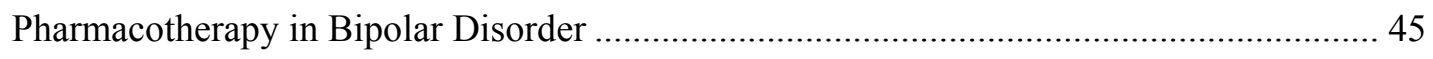

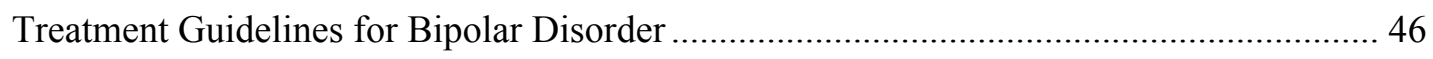

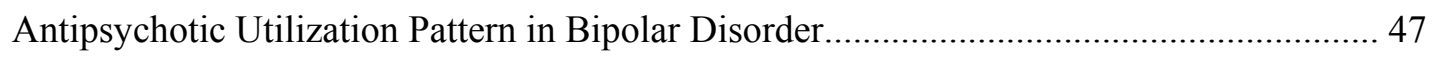

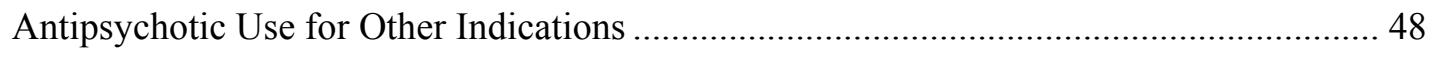

Antipsychotic Therapy and Economic Outcomes in Schizophrenia.................................. 49 
Antipsychotic Therapy and Economic Outcomes in Bipolar Disorder.

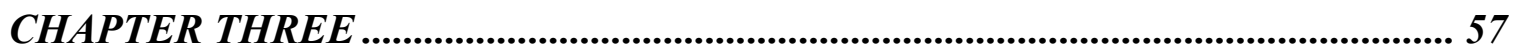

METHODOLOGY .............................................................................................. 57

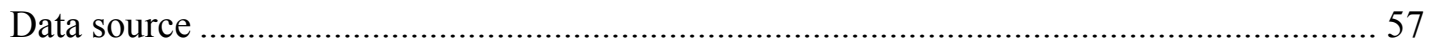

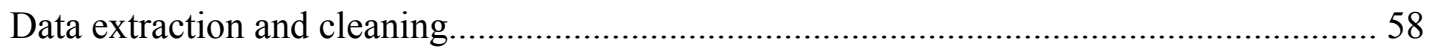

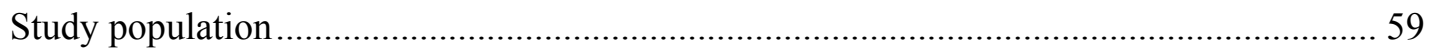

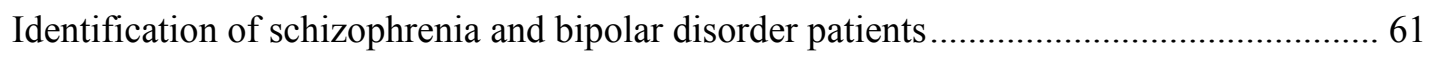

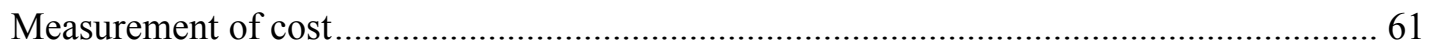

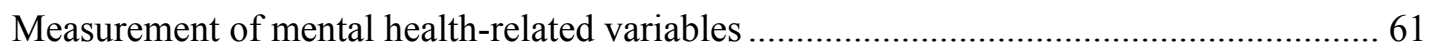

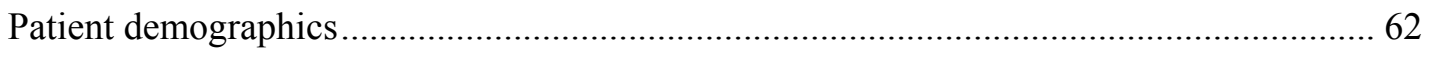

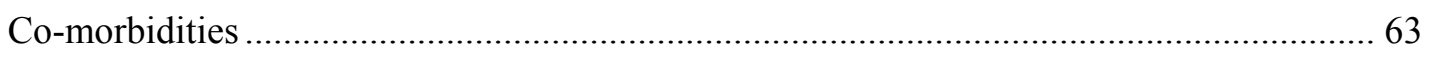

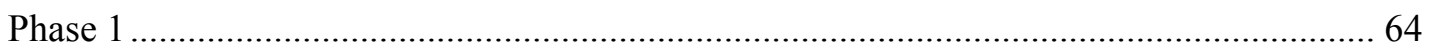

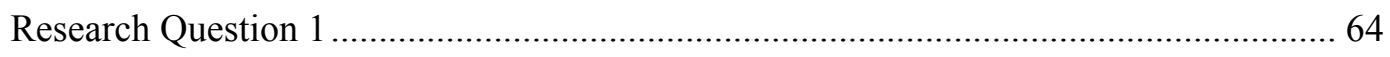

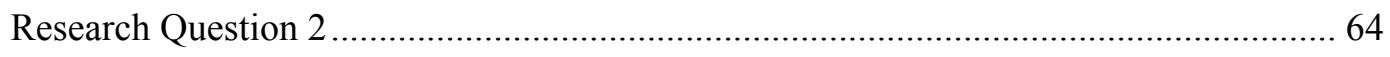

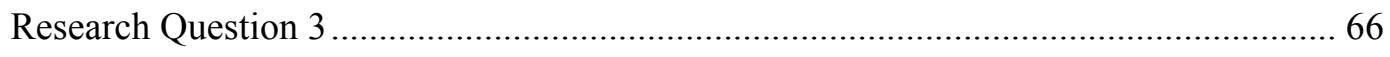

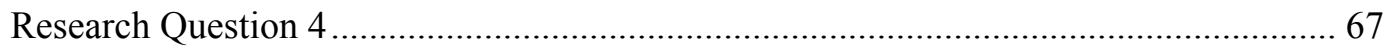

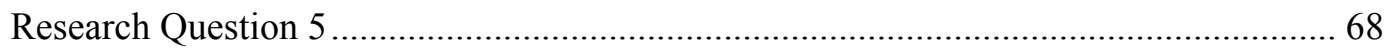

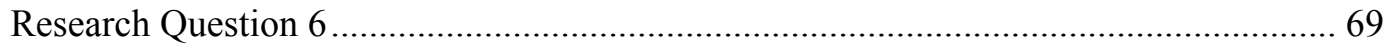

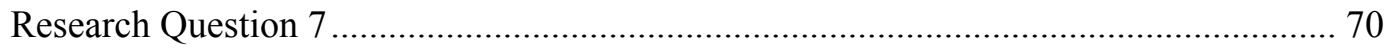

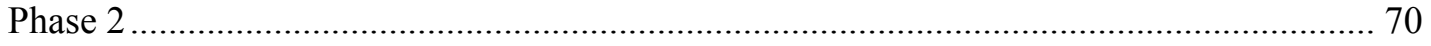

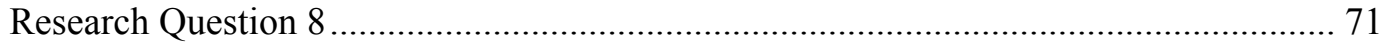

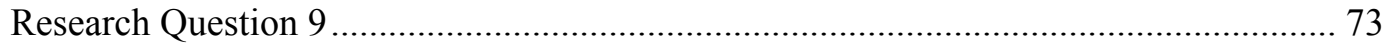

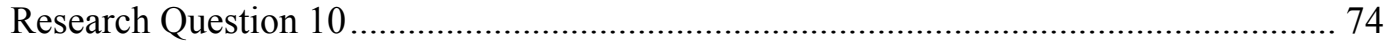

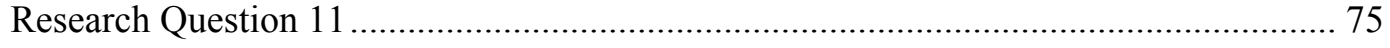

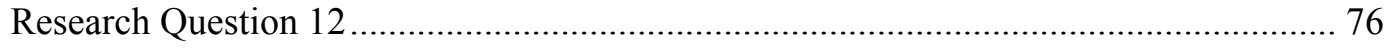

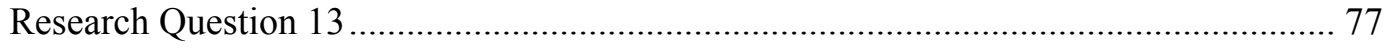




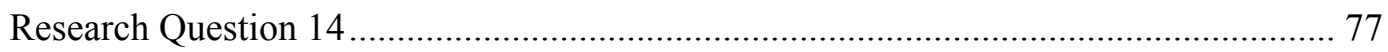

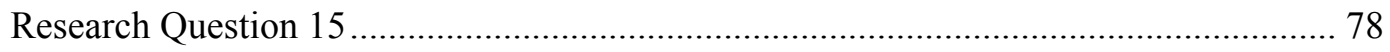

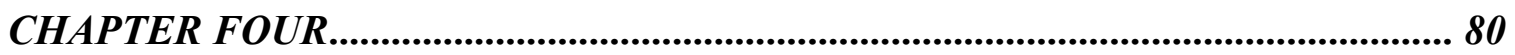

RESULTS AND DISCUSSION ......................................................................................... 80

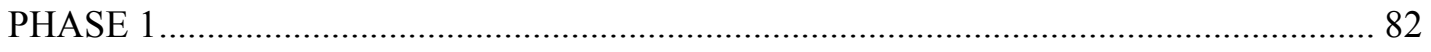

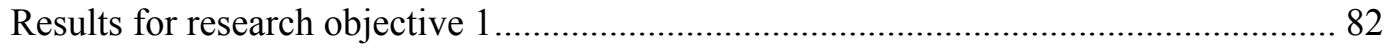

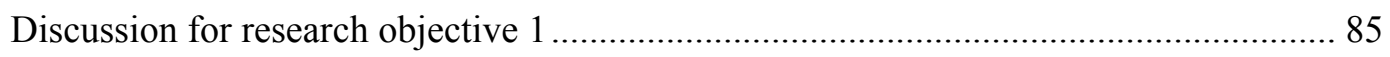

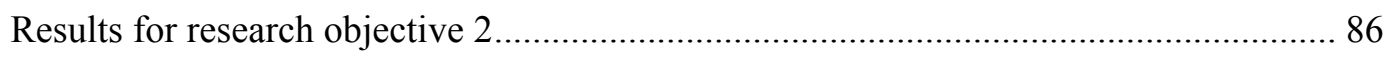

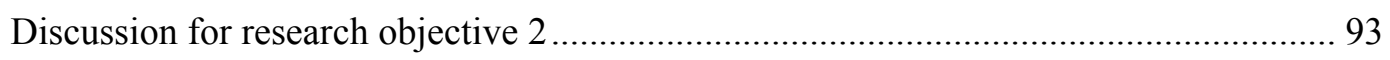

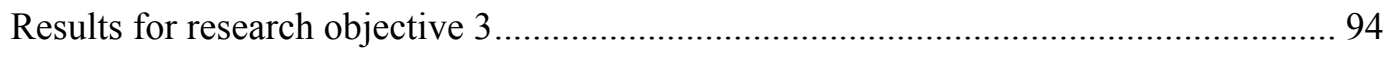

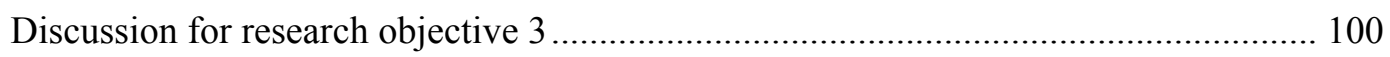

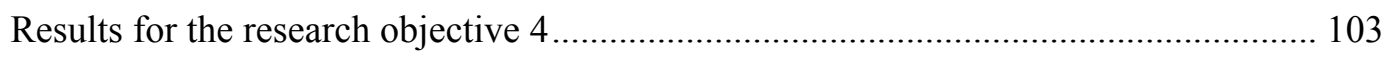

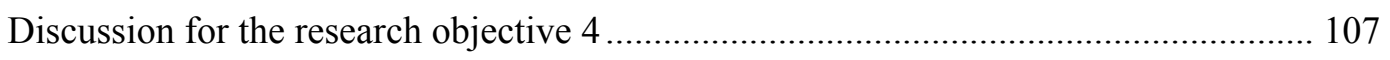

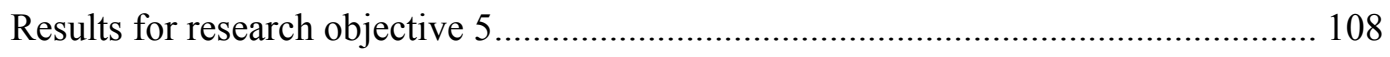

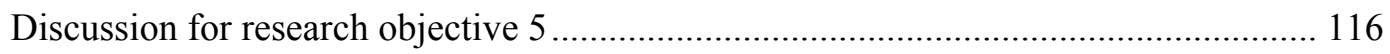

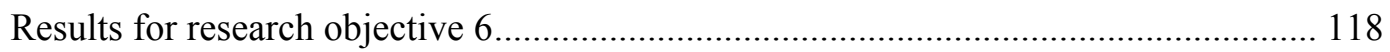

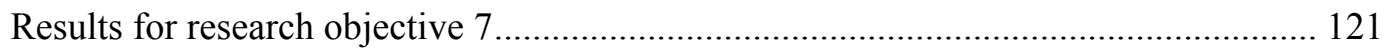

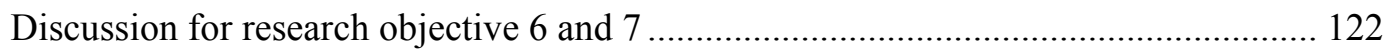

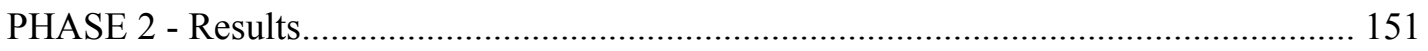

Demographic characteristics of patients initiated on antipsychotics........................... 151

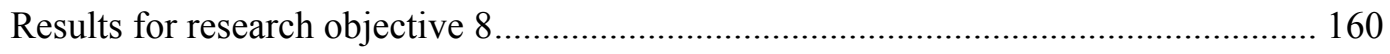

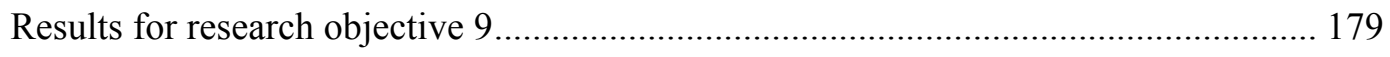

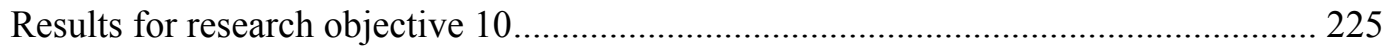

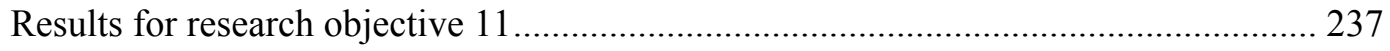

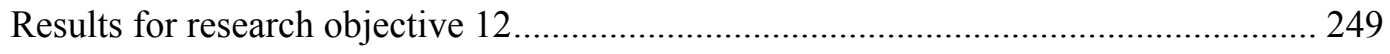

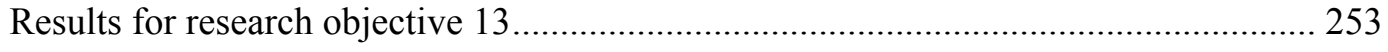




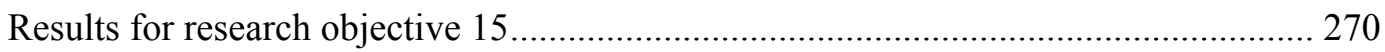

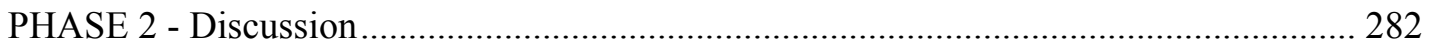

Total and mental healthcare costs for schizophrenia and bipolar disorder patients ....... 282

Mental health-related inpatient utilization and cost ................................................ 285

Mental health-related ER and outpatient utilization and cost..................................... 286

Mental health-related pharmacy utilization and cost.............................................. 287

Index antipsychotic adherence and therapy modification ......................................... 291

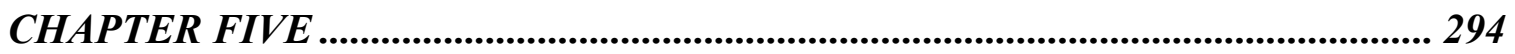

CONCLUSIONS ….................................................................................................................. 294

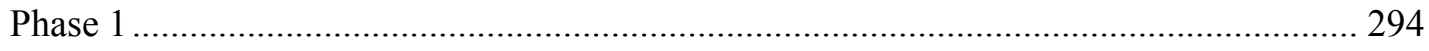

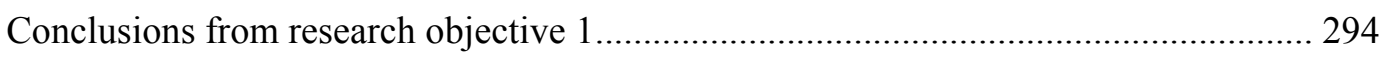

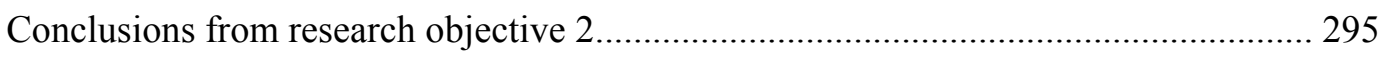

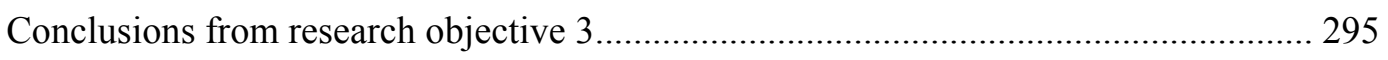

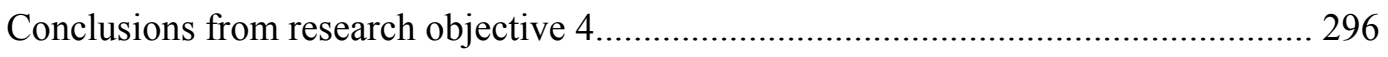

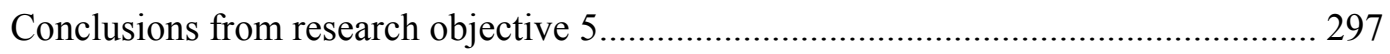

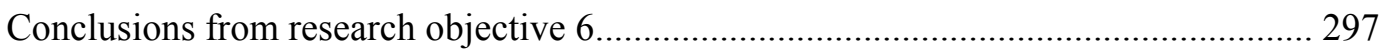

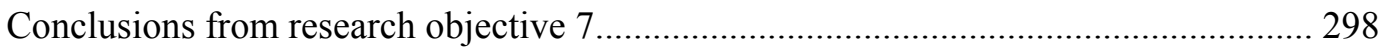

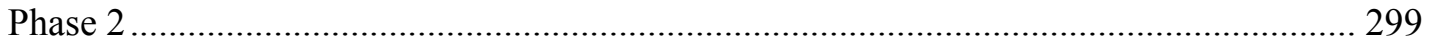

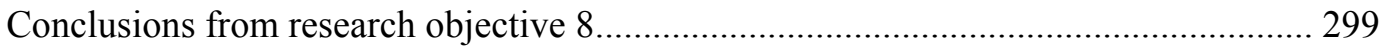

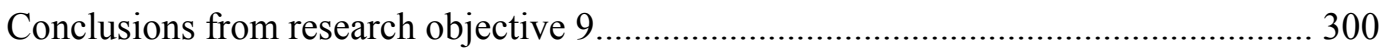

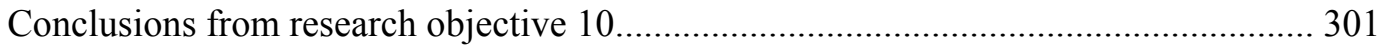

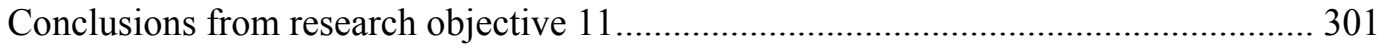

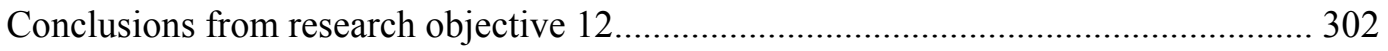

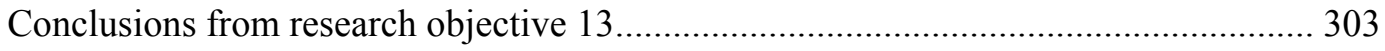

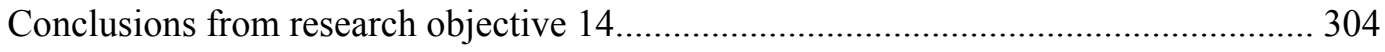

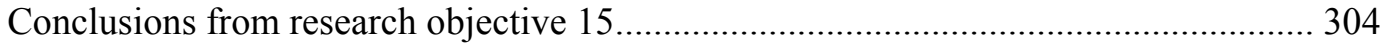




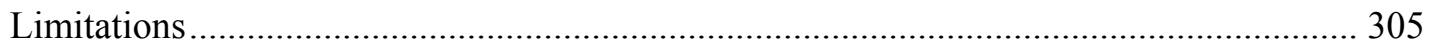

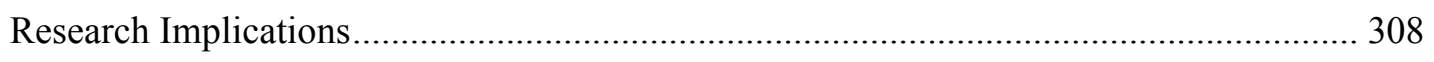

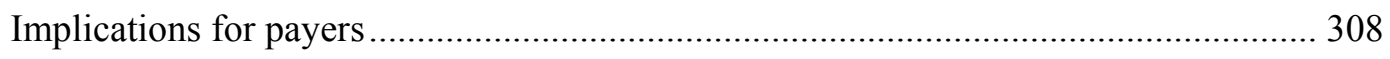

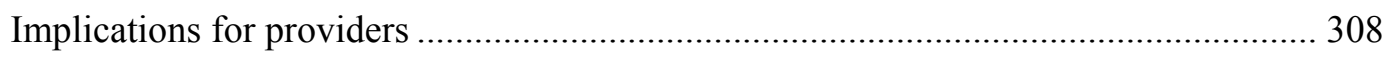

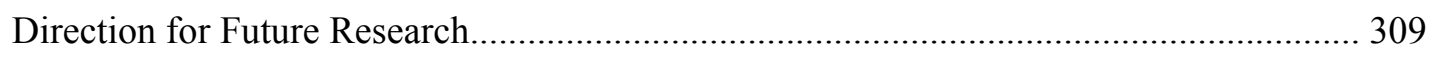

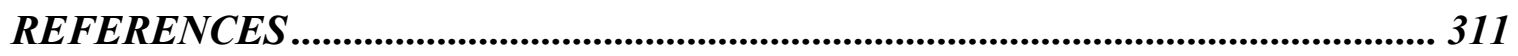

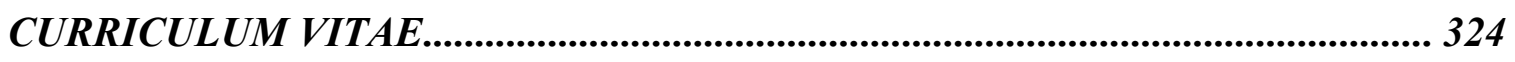




\section{LIST OF TABLES}

Table 1: Application of the selection criteria and the resulting sample size

Table 2a: Annual prevalence rate for schizophrenia in the WV Medicaid (1998-2002)

Table 2b: Annual prevalence rate for bipolar disorder in the WV Medicaid (1998-2002).....

Table 3a: Exploratory analysis of mental health conditions among patients who were initiated on antipsychotics in the West Virginia Medicaid population during the study period.

Table 3b: Exploratory analysis of mental health conditions among patients who were initiated on antipsychotics in the West Virginia Medicaid population during the study period.

Table 3c: Exploratory analysis of mental health conditions among patients who were initiated on antipsychotics in the West Virginia Medicaid population during the study period......

Table 4a: Pattern of antipsychotic use in the 12-month follow-up period among Schizophrenia

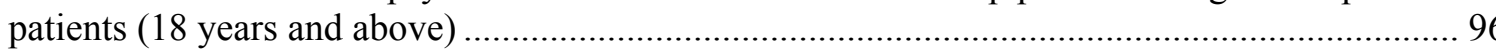

Table 4b: Duration of index antipsychotic prescription use among schizophrenia patients

Table 5: Pattern of antipsychotic use in the 12-month follow-up period among bipolar disorder patients.

Table 6: Study of antipsychotic treatment gaps among schizophrenia patients (18 years and above)

Table 7: Study of antipsychotic treatment gaps among bipolar disorder patients

Table 8: Multinomial logistic regression model determining predictors of pattern of antipsychotic use among schizophrenia patients (first-stage sample selection model)

Table 9: Multinomial logistic regression model determining predictors of pattern of antipsychotic use among bipolar disorder patients (first-stage sample selection model)....

Table 10: Total and mental healthcare costs associated with the different antipsychotic utilization patterns among schizophrenia patients.

Table 11a: Ordinary Least Squares (OLS) regression model for the impact pattern of antipsychotic use on total healthcare cost among schizophrenia patients (second stage sample selection models): Interrupted vs. Adherent therapy.....

Table 11b: Ordinary Least Squares (OLS) regression model for the impact pattern of antipsychotic use on total healthcare cost among schizophrenia patients (second stage sample selection models): Switching vs. adherent therapy

Table 11c: Ordinary Least Squares (OLS) regression model for the impact pattern of antipsychotic use on total healthcare cost among schizophrenia patients (second stage sample selection models): Polytherapy vs. adherent therapy 
Table 12a: Ordinary Least Squares (OLS) regression model for the impact pattern of antipsychotic use on mental healthcare cost among schizophrenia patients (second stage sample selection models): Interrupted vs. adherent therapy.

Table 12b: Ordinary Least Squares (OLS) regression model for the impact pattern of antipsychotic use on mental healthcare cost among schizophrenia patients (second stage sample selection models): Switch vs. adherent therapy.....

Table 12c: Ordinary Least Squares (OLS) regression model for the impact pattern of antipsychotic use on mental healthcare cost among schizophrenia patients (second stage sample selection models): Polytherapy vs. adherent therapy .....

Table 13: Total and mental healthcare costs associated with the different antipsychotic utilization patterns among bipolar disorder patients.

Table 14a: Ordinary Least Squares (OLS) regression model for the impact pattern of antipsychotic use on total healthcare cost among bipolar disorder patients (second stage sample selection models): Interrupted vs. adherent therapy.

Table 14b: Ordinary Least Squares (OLS) regression model for the impact pattern of antipsychotic use on total healthcare cost among bipolar disorder patients (second stage sample selection models): Switching vs. adherent therapy ....

Table 14c: Ordinary Least Squares (OLS) regression model for the impact pattern of antipsychotic use on total healthcare cost among bipolar disorder patients (second stage sample selection models): Polytherapy vs. adherent therapy

Table 15a: Ordinary Least Squares (OLS) regression model for the impact pattern of antipsychotic use on mental healthcare cost among bipolar disorder patients (second stage sample selection models): Interrupted vs. adherent therapy.

Table 15b: Ordinary Least Squares (OLS) regression model for the impact pattern of antipsychotic use on mental healthcare cost among bipolar disorder patients (second stage sample selection models): Switch vs. adherent therapy.....

Table 15c: Ordinary Least Squares (OLS) regression model for the impact pattern of antipsychotic use on mental healthcare cost among bipolar disorder patients (second stage sample selection models): Polytherapy vs. adherent therapy

Table 16: Demographic characteristics of schizophrenia patients (18 years and older) who were initiated on study antipsychotics between January 1, 1999 and December 31, $2001(\mathrm{~N}=999) .154$

Table 17: Demographic characteristics of bipolar disorder patients who were initiated on study antipsychotics between January 1, 1999 and December 31, $2001(\mathrm{~N}=825)$.....

Table 18: Healthcare costs comparison among schizophrenia patients: (Quetiapine versus other antipsychotics)...

Table 19: Multinomial logistic regression model determining predictors of index antipsychotic use among schizophrenia patients (first-stage sample selection model) 
Table 20a: Ordinary Least Squares (OLS) regression model for the impact of index antipsychotic on total healthcare cost among schizophrenia patients (second stage sample selection models):

Olanzapine vs. quetiapine. 166

Table 20b: Ordinary Least Squares (OLS) regression model for the impact of index antipsychotic on total healthcare cost among schizophrenia patients (second stage sample selection models):

Risperidone vs. quetiapine. 168

Table 20c: Ordinary Least Squares (OLS) regression model for the impact of index antipsychotic on total healthcare cost among schizophrenia patients (second stage sample selection models):

Typicals vs. quetiapine.

Table 21a: Ordinary Least Squares (OLS) regression model for the impact of index antipsychotic on mental healthcare cost among schizophrenia patients (second stage sample selection models):

Olanzapine vs. quetiapine.

Table 21b: Ordinary Least Squares (OLS) regression model for the impact of index antipsychotic on mental healthcare cost among schizophrenia patients (second stage sample selection models):

Risperidone vs. quetiapine.

Table 21c: Ordinary Least Squares (OLS) regression model for the impact of index antipsychotic on mental healthcare cost among schizophrenia patients (second stage sample selection models):

Typicals vs. quetiapine

Table 22: Healthcare costs comparison among bipolar disorder patients: (Quetiapine versus other antipsychotics)

Table 23: Multinomial logistic regression model determining predictors of index antipsychotic use among bipolar disorder patients (first-stage sample selection model).

Table 24a: Ordinary Least Squares (OLS) regression model for the impact of index antipsychotic on total healthcare cost among bipolar disorder patients (second stage sample selection models):

Olanzapine vs. quetiapine.

Table 24b: Ordinary Least Squares (OLS) regression model for the impact of index antipsychotic on total healthcare cost among bipolar disorder patients (second stage sample selection models):

Risperidone vs. quetiapine.

Table 24c: Ordinary Least Squares (OLS) regression model for the impact of index antipsychotic on total healthcare cost among bipolar disorder patients (second stage sample selection models):

Typicals vs. quetiapine

Table 25a: Ordinary Least Squares (OLS) regression model for the impact of index antipsychotic on mental healthcare cost among bipolar disorder patients (second stage sample selection models): Olanzapine vs. quetiapine.

Table 25b: Ordinary Least Squares (OLS) regression model for the impact of index antipsychotic on mental healthcare cost among bipolar disorder patients (second stage sample selection models): Risperidone vs. quetiapine. 
Table 25c: Ordinary Least Squares (OLS) regression model for the impact of index antipsychotic on mental healthcare cost among bipolar disorder patients (second stage sample selection models): Typicals vs. quetiapine.

Table 26a: Logistic regression model for the impact of index antipsychotic on having a mental health-related hospitalization episode among schizophrenia patients

Table 26b: Ordinary Least Squares (OLS) regression model for the impact of index antipsychotic on mental healthcare-related hospitalization cost among schizophrenia patients

Table 27a: Logistic regression model for the impact of index antipsychotic on having a mental health-related ER episode among schizophrenia patients

Table 27b: Ordinary Least Squares (OLS) regression model for the impact of index antipsychotic on mental healthcare-related ER cost among schizophrenia patients. 206

Table 28: Ordinary Least Squares (OLS) regression model for the impact of index antipsychotic on mental healthcare-related pharmacy cost among schizophrenia patients 208

Table 29: Ordinary Least Squares (OLS) regression model for the impact of index antipsychotic on mental healthcare-related outpatient cost among schizophrenia patients.....

Table 30a: Logistic regression model for the impact of index antipsychotic on having a mental health-related hospitalization episode

Table 30b: Ordinary Least Squares (OLS) regression model for the impact of index antipsychotic on mental healthcare-related hospitalization cost among bipolar disorder patients ..... 214

Table 31a: Logistic regression model for the impact of index antipsychotic on having a mental health-related ER episode among bipolar disorder patients 216

Table 31b: Ordinary Least Squares (OLS) regression model for the impact of index antipsychotic on mental healthcare-related ER cost among bipolar disorder patients 219

Table 32: Ordinary Least Squares (OLS) regression model for the impact of index antipsychotic on mental healthcare-related pharmacy cost among bipolar disorder patients

Table 33: Ordinary Least Squares (OLS) regression model for the impact of index antipsychotic on mental healthcare-related outpatient cost

Table 34: Mental health-related hospitalization comparison (Quetiapine versus other antipsychotics) among schizophrenia patients

Table 35: Negative binomial model for the impact of index antipsychotic therapy of number of mental health-related hospitalization among schizophrenia patients 228

Table 36: Cox Proportional hazard model for the impact of index antipsychotic therapy on time to the first mental health-related hospitalization among schizophrenia patients.... 231

Table 37: Mental health-related hospitalization comparison (Quetiapine versus other antipsychotics) among bipolar disorder patients 
Table 38: Negative binomial model for the impact of index antipsychotic therapy of number of mental health-related hospitalization among bipolar disorder patients

Table 39: Cox Proportional hazard model for the impact of index antipsychotic therapy on time to the first mental health-related hospitalization among bipolar disorder patients.

Table 40: Mental health-related ER visits comparison (Quetiapine versus other antipsychotics) among schizophrenia patients.

Table 41: Zero-inflated poisson regression model for the impact of index antipsychotic therapy of number of mental health-related ER visit among schizophrenia patients

Table 42: Mental health-related ER visits comparison (Quetiapine versus other antipsychotics) among bipolar disorder patients

Table 43: Zero-inflated poisson regression model for the impact of index antipsychotic therapy of number of mental health-related ER visit among bipolar disorder patients

Table 44: Physician office-visits, psychotherapy, and medication management psychotherapy sessions comparison (Quetiapine versus other antipsychotics) among schizophrenia patients... 250

Table 45: Negative binomial model for the impact of index antipsychotic therapy of number of mental health-related physician visit among schizophrenia patients. 251

Table 46: Physician office-visits, psychotherapy, and medication management psychotherapy sessions comparison (Quetiapine versus other antipsychotics) among bipolar disorder patients 254

Table 47: Negative binomial model for the impact of index antipsychotic therapy of number of mental health-related physician visit among bipolar disorder patients 255

Table 48: Psychiatric medication use comparison (Quetiapine versus other antipsychotics) among schizophrenia patients 257

Table 49: Psychiatric medication use comparison (Quetiapine versus other antipsychotics) among bipolar disorder patients 261

Table 50: Univariate comparison of medication possession ratio (mpr) among schizophrenia patients.

Table 51: Ordinary Least Squares (OLS) regression model for adherence to the index antipsychotic therapy among schizophrenia patients

Table 52: Univariate comparison of medication possession ratio (mpr) among bipolar disorder patients.

Table 53: Ordinary Least Squares (OLS) regression model for adherence to the index antipsychotic therapy among bipolar disorder patients .... 268

Table 54: Therapy modification among schizophrenia patients 272

Table 55: Cox Proportional hazard model for the impact of index antipsychotic therapy on time to discontinuation/switch or polytherapy among schizophrenia patients 274 
Table 56: Therapy modification among bipolar disorder patients...

Table 57: Extended Cox Proportional hazard model for the impact of index antipsychotic therapy on time to discontinuation/ switch or polytherapy among bipolar disorder patients.................. 279 


\section{LIST OF FIGURES}

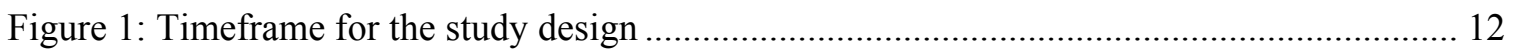

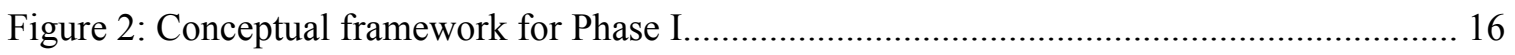

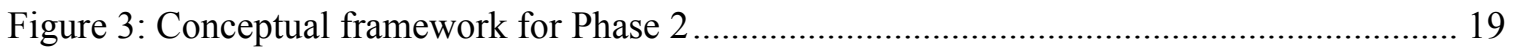

Figure 4: Kaplan Meier Survival Curves for time to first mental health-related hospitalization

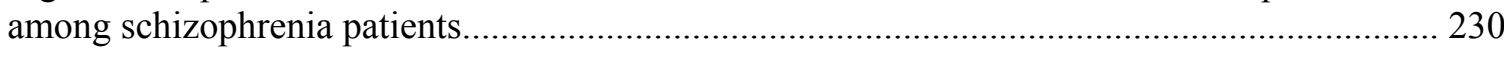

Figure 5: Kaplan Meier Survival Curves for time to first mental health-related hospitalization among bipolar disorder patients

Figure 6: Kaplan-Meier Survival Curves for the time to modification of index antipsychotic prescription in schizophrenia patients

Figure 7: Kaplan-Meier Survival Curves for time to modification of index antipsychotic prescription in bipolar disorder patients 


\section{CHAPTER ONE}

\section{INTRODUCTION}

\section{Schizophrenia}

Schizophrenia is a complex psychiatric disorder affecting about $1.1 \%$ of the population. Despite the low prevalence of the disease, schizophrenia is the most expensive psychiatry disorder to treat (Knapp, 1997). Schizophrenia costs constitute $2.5 \%$ of total healthcare expenditures. In 1991, direct and indirect costs associated with schizophrenia were estimated to be $\$ 18.6$ billion and $\$ 46.5$ billion, respectively. Hospitalizations are responsible for almost $70 \%$ of costs, drug costs for almost $10 \%$, and outpatient costs usually make up the rest of the $20 \%$ (Revicki, 1997). Annual total healthcare costs per schizophrenia patient were estimated to range between $\$ 16,000$ and \$57,000 in 1999 (Mauskopf, David, Grainger, \& Gibson, 1999).

\section{Bipolar Disorder}

The lifetime prevalence of bipolar disorder in the US population is about $1.2 \%$ to $1.6 \%$ (Kessler et al., 1994). The estimated economic burden of the disorder is about $\$ 45$ billion. The direct costs are estimated at $\$ 7.6$ billion and indirect costs at $\$ 38$ billion. About $60-70 \%$ of direct costs were attributable to inpatient costs (Wyatt \& Henter, 1995). Medication costs constitute about $2 \%$ of direct costs. The annual medication cost per treated patient was about $\$ 548$ (Wyatt et al., 1995). About $75 \%$ of bipolar patients undergo at least one hospitalization during the course of their illness (Lish, DimeMeenan, Whybrow, Price, \& Hirschfeld, 1994). About 10\% of inpatient healthcare in US is utilized by bipolar patients (Narrow, Regier, Rae, Manderscheid, \& Locke, 1993). 
Costs for patients with bipolar disorder is higher than costs for patients treated for depression or diabetes (Simon \& Unutzer, 1999).

\section{Antipsychotic Treatment Patterns}

Pharmacotherapy of schizophrenia involves use of conventional antipsychotics and relatively newer atypical antipsychotics. With the advent of newer atypical antipsychotics over the last decade, the treatment pattern of antipsychotic therapy is changing. Various studies conducted in different healthcare settings and time periods

have reported this trend. A study by Williams et al using the Regenstrief Medical Record System reported only $12 \%$ atypical antipsychotic use among schizophrenia patients in 1995. About $25 \%$ of patients switched medications and $90 \%$ augmented medications during a 12-month period (Williams, Johnstone, Kesterson, Javor, \& Schmetzer, 1999). A chart review of 83 outpatients showed that patients who switched antipsychotics made greater use of psychiatric services (Tempier \& Pawliuk, 2003). McCombs et al used the claims data from the California Medicaid program (Medi-Cal) from 1987 to 1996 to study antipsychotics utilization patterns in terms of no drug therapy for over 1 year; delayed onset of antipsychotic drug therapy; and, switches in antipsychotic drugs within 1 year. During the time period of the study, they found that about $98 \%$ of patients were on conventional antipyschotics. About $47 \%$ of patients switched or augmented their therapy within one year. Only $11.6 \%$ of patients received continuous antipsychotic therapy for one year (McCombs, Nichol, Stimmel, Shi, \& Smith, 1999b). These studies used data earlier than 1998 during which atypical antipsychotics were not widely used. Drug use pattern of antipsychotics has probably changed since this study as newer atypical antipsychotics have entered market and are being more widely used. 
Until recently, atypical antipsychotics were not approved for use for bipolar patients though their use was prevalent in clinical practice (Licht et al., 1994; Shattell \& Keltner, 2004). Pattern of antipsychotic use among bipolar patients has been less studied. A meta-analysis by Tohen et al revealed that about $84.7 \%$ of bipolar patients received typical antipsychotics. About $53.8 \%$ of typical antipsychotic use was as monotherapy. About $47.4 \%$ of typical antipsychotic use was as an adjunct with a mood stabilizer (Tohen et al., 2001). A study by Russo et al reported that typical antipsychotics were used by $16.4 \%$ of patients and atypical antipsychotics by $12.4 \%$ of bipolar patients between 1994 and 1998. The study also found that patients initiated on antipsychotics continued on the therapy for more than 12 months, while patients initiated on anticonvulsants or antidepressants were more likely to discontinue or switch to another medication class (Russo, Smith, Dirani, Namjoshi, \& Tohen, 2002).

\section{Antipsychotic polypharmacy}

Literature reveals widespread practice of antipsychotic polypharmacy. Various studies have reported prevalence of antipsychotic polypharmacy in clinical practice despite the lack of clinical evidence supporting such practice. Analyzing prescription records of patients hospitalized between 1995 and 2000, McCue et al reported about 16\% of schizophrenia patients on multiple antipsychotics. However, the study claims that polypharmacy was linked with shorter length of hospitalization and fewer adverse effects (McCue, Waheed, \& Urcuyo, 2003). Procyshyn et al found that 27.5\% of schizophrenic patients discharged from a tertiary psychiatric clinic were prescribed multiple antipsychotics. As compared to monotherapy, antipsychotic polytherapy was linked with greater use of anticholinergics (Procyshyn, Kennedy, Tse, \& Thompson, 2001). Clark et 
al reported that antipsychotic polytherapy has increased from $5.7 \%$ in 1995 to $35.6 \%$ in 1999 among schizophrenia patients (Clark, Bartels, Mellman, \& Peacock, 2002). A retrospective case-control study of multiple versus single antipsychotic treatment in psychiatric inpatients revealed that multiple antipsychotic therapy is associated with more adverse effects and hospitalizations but no clinical benefit (Centorrino et al., 2004). A retrospective survey of discharge medications at a tertiary care psychiatric hospital found that antipsychotic polytherapy was very common in schizoaffective disorder (49.3\%), followed by schizophrenia (44.7\%), bipolar disorder (29.9\%), and psychosis not otherwise specified (22.5\%) (Procyshyn \& Thompson, 2004). More data on polytherapy practices in bipolar disorder is lacking. More studies are needed to develop guidelines to aid clinicians and health policy decision makers regarding antipsychotic polytherapy.

\section{Antipsychotic Use for Other Indications}

Though atypicals were introduced for the management of schizophrenia, they are increasingly being used for other indications. A cross-sectional analysis of nursing home residents from Jan. 1, 1999, to Jan. 31, 2000 revealed that atypical antipsychotic were generally used in Parkinson's disease, depression, Alzheimer's disease, and nonAlzheimer dementia (Liperoti et al., 2003). Antipsychotic use has also substantially increased for attention-deficit/hyperactivity disorder, conduct disorder, and affective disorders among patients aged 2 to 18 years (Cooper, Hickson, Fuchs, Arbogast, \& Ray, 2004). Though already prevalent to some extent in clinical practice, use of certain atypical antipsychotics for bipolar disorder was recently approved by FDA. However, antipyschotics use for conditions other than schizophrenia and bipolar disorders is not clearly supported by clinical studies and are not approved by FDA. 


\section{Antipsychotic Therapy and Economic Outcomes}

As schizophrenia and bipolar disorders are associated with the extensive use of inpatient services, treatments that can reduce inpatient utilization may decrease the overall costs and improve the quality of life for patients, their families, and society. Though more costly than typical antipsychotics, atypical antipsychotics offer better outcomes and may be cost-effective in the long run. Despite the increase in pharmacy costs, atypicals have been shown to offset inpatient and outpatient costs as compared to typical antipsychotics (Csernansky \& Schuchart, 2002; Gianfrancesco, Durkin, Mahmoud, \& Wang, 2002; Gibson, Damler, Jackson, Wilder, \& Ramsey, 2004; Nightengale, Garrett, Waugh, Lawrence, \& Andrus, 1998).

Each atypical antipsychotic has its unique pharmacologic profile and is priced differently. However, there are very few pharmacoeconomic comparisons among the atypical antipsychotics. To date, most of the published studies have compared only risperidone and olanzapine. Relative advantage offered by one drug as compared to the other has differed depending upon study population, outcome measures, research and statistical design. A prospective observational study comparing olanzapine and risperidone showed that olanzapine had a lower risk of psychiatric hospitalization than risperidone-treated schizophrenia patients within the first year (Ascher-Svanum, Zhu,

Faries, \& Ernst, 2004). A retrospective analysis comparing total health care costs for patients initiated on risperidone or olanzapine between March 1997 and March 1999 reported decline in costs for risperidone-treated patients and increase in costs for olanzapine-treated patients (Fuller, Shermock, Secic, Laich, \& Durkin, 2002). An analysis of Michigan Medicaid claims from January 1995 through September 1998 
revealed that total costs were not significantly different between olanzapine, risperidone and haloperidol treated schizophrenia patients. However, olanzapine was associated with lower inpatient costs and risperidone was associated with greater persistence with therapy (Gibson et al., 2004). The results from the health economic data from the Risperidone Olanzapine Drug Outcomes studies in Schizophrenia (RODOS) program show that olanzapine was associated with higher total costs as compared to risperidone without any clinical benefit (Kasper, Jones, \& Duchesne, 2001). A study examining inpatient data of 789 patients with schizophrenia or schizoaffective disorder initiated on risperidone or olanzapine reported higher discharge rate and lower drug costs among risperidone treated patients (Kelly, Nelson, Love, Yu, \& Conley, 2001). Another study showed that patients initiated on olanzapine were prescribed anti-parkinson drugs less frequently (Zhao, 2002). Costs and utilization among patients initiated on olanzapine or risperidone between January 1, 1997 and August 31, 1998 were compared using Texas Medicaid data. Results showed no significant differences among schizophrenia-related costs but the total medical costs were lower among patients initiated in olanzapine (Rascati, Johnsrud, Crismon, Lage, \& Barber, 2003).

There is only one published economic analysis that compares risperidone, olanzapine and quetiapine in real world settings. This study evaluated only the pharmacy costs in an acute care inpatient setting. The study reports lower pharmacy cost for risperidone and quetiapine as compared to olanzapine (Mladsi et al., 2004). There are no other published studies comparing quetiapine, a relatively newer atypical antipsychotic, with other atypical antipsychotics in real world settings. A clinical decision modeling study comparing atypical antipsychotics in terms of non-compliance, relapse and cost 
among schizophrenia patients showed higher non- compliance, relapses, and incremental cost among risperidone and olanzapine treated patients as compared to quetiapine (Mortimer, Williams, \& Meddis, 2003). The Partial Responders International schizophrenia Evaluation (PRIZE) clinical trial comparing quetiapine and haloperidol using a Markov model showed that though quetiapine has the higher acquisition cost, it can lower costs for other medications, hospitalization, and other medical services (Tilden, Aristides, Meddis, \& Burns, 2002).

Literature on health care cost and utilization outcomes of antipsychotic therapy in bipolar disorder is lacking. Economic outcomes from a randomized clinical trial of olanzapine treatment in patients diagnosed with bipolar I disorder with mania showed cost saving of almost $\$ 900$ per month during the 49 weeks of olanzapine therapy. These cost savings were mainly attributed to reductions in in-patient costs. Thus, it is possible that olanzapine and other atypicals may prove to be cost-effective in bipolar disorders if similar results can be shown in non-clinical trial populations (Namjoshi et al., 2002). There is only one published study comparing economic outcomes of different antipsychotics in bipolar disorder. This study reports that mental-health related costs were higher for olanzapine as compared to risperidone and quetiapine. There was no significant difference in costs between risperidone and quetiapine (Gianfrancesco, Pesa, \& Wang, 2005).

\section{STATEMENT OF PROBLEM}

Several atypical antipsychotics have entered the market since the last decade. Evaluating utilization pattern and the value added to the therapy by these expensive 
agents is becoming more important as their use continues to expand. There is lack of data on the population demographics, treatment patterns, utilization and costs of these newer drugs in general population. There is a need to document that antipsychotic use is not restricted to schizophrenia in clinical practice. The purpose of the present study is to link the antipsychotic therapy patterns with healthcare utilization and costs in the West Virginia Medicaid population. As previous studies have used data until 1998, there is a need for observational studies evaluating changes in utilization patterns with introduction of newer drugs.

Antipsychotic polypharmacy is prevalent in clinical practice despite the lack of scientific evidence. The practice probably results due to past experience or intuitive judgment of practicing physicians. Studying the healthcare outcomes and economic consequences of this practice can be helpful in developing guidelines for antipsychotic polypharmacy. They can also support the need for clinical trials evaluating the decision to use antipsychotic polypharmacy in some patients.

State Medicaid is the single largest payer for mental health care. In 1996, Medicaid spent about $\$ 12.6$ billion on mental health care (Hogan, 1999). The burden of mental disorders such as schizophrenia and bipolar disorder is very high due to the chronic nature, hospitalizations, comorbidities and disabilities associated with these diseases. Due to very high unemployment among patients with such mental disorders, the state Medicaid bears the greatest impact of this disease (Hogan, 1998; Hogan, 1999). Atypical antipsychotics are among the ten most expensive medications for Medicaid (Clark et al., 2002). If the pharmacotherapy is effective in controlling the disease symptoms and severity, it can reduce the need for medical services and offset costs. 
Though newer antipsychotics have high acquisition costs, they offer advantages in treatment such as better adherence and reduced hospitalizations. From a payer's perspective, it is important to determine which atypical antipsychotic would be most effective in reducing utilization and generating savings in total healthcare expenditures in their patient population.

Though the total medical cost is the most relevant economic outcome for formulary decision-making purposes, the differences in total cost depend upon differences in the use of inpatient services, physician services, concomitant medications, changes in treatment or treatment discontinuation rates, and relapse rates. These components of healthcare costs need to be studied to gain better understanding of costs associated with antipsychotic use. Very few studies in the literature have looked at the use of antipsychotics in bipolar disorder. This study aims to provide more information in terms of describing utilization pattern and economic comparisons of antipsychotics in bipolar disorder.

Randomized clinical trials have strict inclusion and exclusion criteria for patient enrollment. Their findings cannot be generalized to the real world settings where the patient population and treatment pattern is more diverse and complex. Use of WV Medicaid data will allow us to study our objectives in a naturalistic setting and with larger sample sizes.

\section{CONCEPTUAL FRAMEWORK}

In phase I, the study will describe different patterns of antipsychotic use in schizophrenia and bipolar disorder patients. Predictors of different pattern of use will be 
determined. The effect of different patterns of use on utilization of healthcare services and costs will be evaluated. The goal of phase II is to compare risperidone, olanzapine, quetiapine and typical antipsychotics on multiple healthcare outcomes such as healthcare costs, hospitalizations, emergency room visits, outpatient visits, concomitant drug use, and adherence and persistence with the index drug,

The rationale for the use of Medicaid data, sample selection criteria, identification of study cohorts, outcomes evaluation and sample selection bias adjustment is provided below.

\section{Selection of Database}

Administrative claims database are usually large longitudinal databases developed for billing and payment purposes by the health plans. They contain medical and pharmacy claims records of the health plan subscribers that can be used for research purposes. Studies using retrospective databases use more relaxed exclusion criteria as compared to randomized clinical trials and have greater generalizability. Claims database studies are a good alternative if the relevant clinical trial has not been conducted or cannot be conducted for ethical reasons. Claims database studies are less costly and more reflective of real world setting. These databases offer large population size and can provide sufficient sample size to give adequate power to the study. They are less susceptible to Hawthorne effect (improvement of performance under observation) as the providers and patients are not contacted (Motheral \& Fairman, 1997; Strom, 2005).

Medicaid offers comprehensive prescription and health benefits and likelihood of out-of system healthcare use among Medicaid population is rare. Due to very high unemployment rate among schizophrenia and bipolar patients, prevalence and burden of 
these diseases in Medicaid is high (Hogan, 1998; Hogan, 1999). In addition, as the actual amount paid for each claim is present in the Medicaid data, it is a valuable resource to study direct costs and utilization of various healthcare components such as hospitalizations, emergency room visits, physician visits and prescriptions among schizophrenia and bipolar patients.

\section{Sample Selection}

West Virginia Medicaid maintains data on patient-level demographic information, medical and prescription drug claims for its recipients. West Virginia Medicaid data for the period January 1, 1998 to December 31, 2002 will be used for the purpose of the study. Schizophrenia and bipolar disorder patients can be identified from this data on basis of ICD-9-CM codes. All paid claims information on patients receiving at least two prescriptions of antipsychotics from January 1, 1999 to December 31, 2001 will be extracted. This period will be defined as the index period. Index date will be the first date of paid claim during the index period for the antipsychotic. Patients should have at least 12 months of continuous enrollment with the West Virginia Medicaid before and after the index date. The patients should not have any claims of an antipsychotic medication during a 90 days period before the index date to allow for a 'wash-out' period before considering the index prescription as initiation of antipsychotic therapy. Patients who were 65 years or older will be excluded as they have Medicare as their primary payer for health services. Managed care recipients will be excluded as they do not have all their utilization information in the Medicaid claims. As clozapine is indicated for treatment refractory schizophrenia, patients initiated on clozapine will be excluded. Figure 1 provides the timeframe and study design. 
Figure 1: Timeframe for the study design

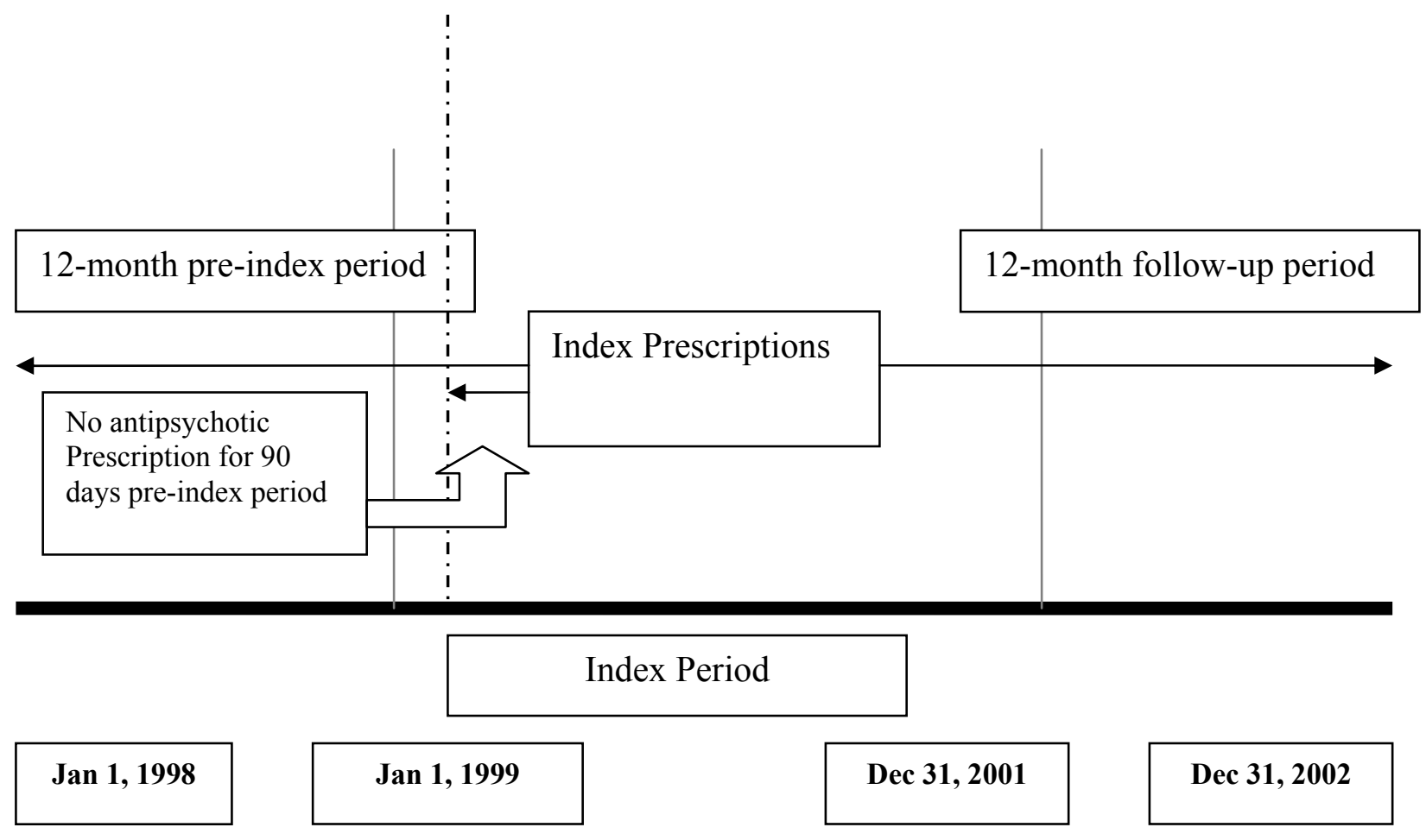




\section{Phase I}

\section{Patterns of antipsychotic Use}

The phase I of the study aims to evaluate the pattern of use of antipsychotic in schizophrenia and bipolar disorder patients. All prescription claims after initiation of antipsychotic will be reviewed to determine the pattern of use of antipsychotic.

The pattern of antipsychotic use in schizophrenia will be categorized into following mutually exclusive categories:

1) Antipsychotic Polytherapy (patients who are receiving two or more antipsychotics)

2) Antipsychotic Monotherapy

a) Switching (patients who change the index antipsychotic to another antipsychotic)

b) Interrupted Therapy (patients receiving less than $80 \%$ days supply of index antipsychotic in the post-index period)

c) Continuous Therapy (patients receiving at least $80 \%$ days supply of index antipsychotic in the post index period)

In addition, the pattern of antipsychotic use will also be studied in terms of:

1) Stabilization on initiating antipsychotic therapy

2) Gaps in antipsychotic therapies that indicate the missing days of antipsychotic therapy in the post-index period.

1) Stabilization on initiating antipsychotic therapy

Proportion of patients who continued on index antipsychotic beyond the trial duration (based on recommendations by the Texas Medication Algorithm Project (TMAP) guidelines) will be determined (Miller et al., 1999). This indicates that the 
patients responded to the index antipsychotic therapy during the trial duration and were continued on the index antipsychotic.

TMAP guidelines recommend trial duration of $3-10$ weeks (including a week of titration to therapeutic dose) after initiation of an antipsychotic before switching antipsychotics. In this study, trial duration of 90 days after initiation of antipsychotic was selected as cut-off to classify the patients who continued on index antipsychotics for 3 months or more and the patients who changed or discontinued the index antipsychotic within 3 months. The 70 days trial duration recommended by TMAP was extended to 90 days in our study based on expert opinion estimating usual physician practices and to allow for some gaps in refills by the patient.

In addition, the pattern of antipsychotic use in bipolar disorder will be categorized into following mutually exclusive categories:

1) Antipsychotic Polytherapy (patients who are receiving two or more antipsychotics)

2) Antipsychotic Monotherapy

a) Switching (patients who change the index antipsychotic to another antipsychotic)

b) Interrupted Therapy (patients receiving less than $80 \%$ days supply of index antipsychotic in the post-index period)

c) Continuous Therapy (patients receiving at least $80 \%$ days supply of index antipsychotic in the post index period)

Pattern of antipsychotic use will also be studied in terms of gaps in antipsychotic therapy. 


\section{Evaluating effect of patterns of use on healthcare cost and utilization}

Treatment pattern of antipsychotic therapy is changing with the introduction of newer antipsychotics. Switching between different antipsychotics is common (McCombs et al., 1999b; Tempier et al., 2003; Williams et al., 1999). Literature also reveals that there is considerable prevalence of antipsychotic polypharmacy in clinical practice settings (Clark et al., 2002; McCue et al., 2003). Various factors such as disease severity, multiple comorbidities, antipsychotic type and patient characteristics can be responsible for existence of different pattern of use for antipsychotics. Also, pattern of antipsychotic use can affect healthcare utilization and costs. It has been shown in some studies that antipsychotic polypharmacy or switching is associated with greater adverse affects, hospitalizations and psychiatric service utilization (Centorrino et al., 2004; Clark et al., 2002; McCue et al., 2003; Tempier et al., 2003). It is likely that patients with polytherapy are more severe than patients on monotherapy. Our approach is to adjust for the sample selection bias that may exist between the different patterns of use groups prior to determining the relationship between patterns of use and healthcare outcomes. Figure 2 presents the conceptual framework regarding relationship between various factors that can influence pattern of antipsychotic therapy in the post-index period. It also gives relationship between patterns of antipsychotic use, costs, and utilization. 
Figure 2: Conceptual framework for Phase I

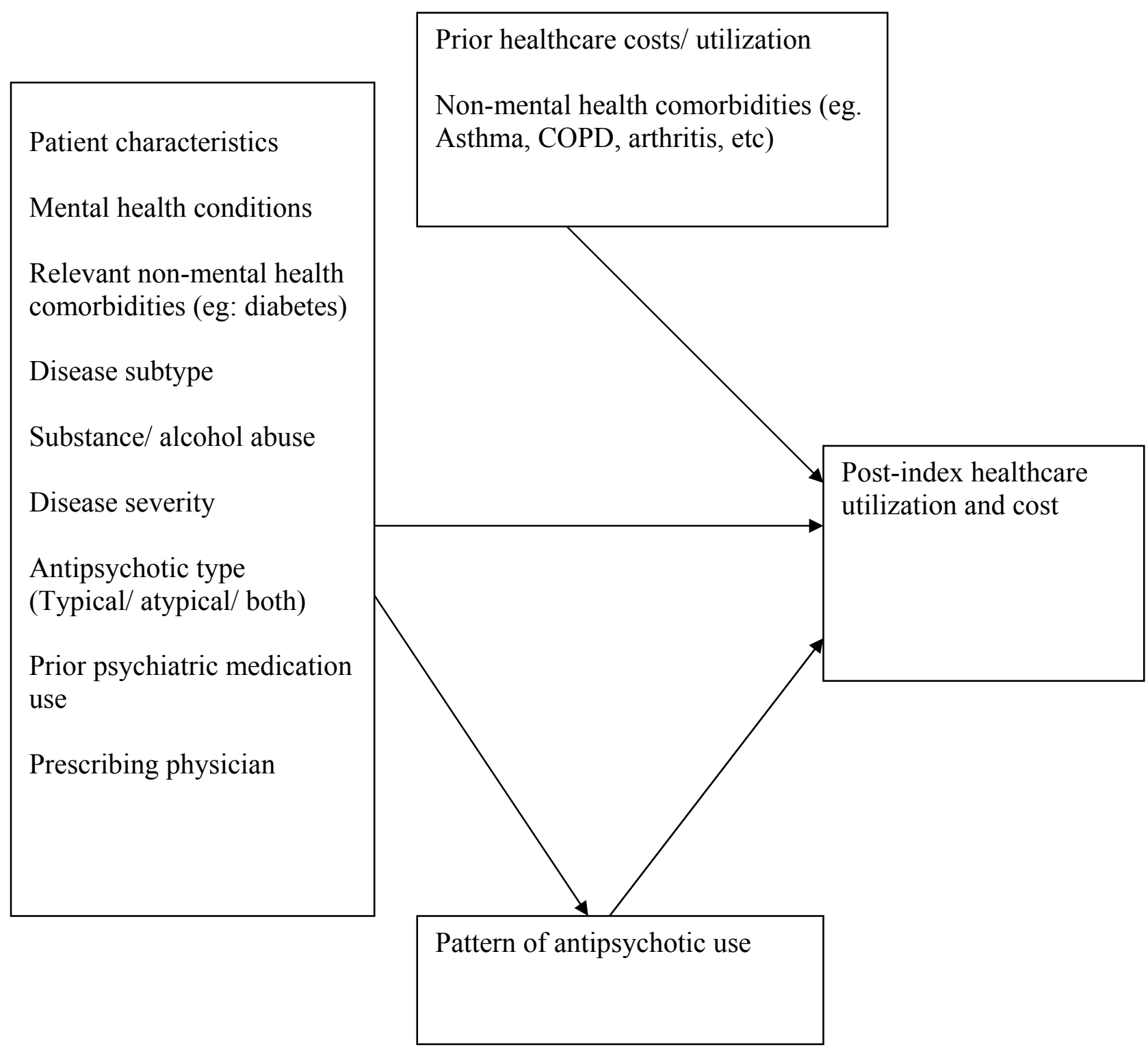




\section{Phase II}

\section{Antipsychotic therapy and healthcare utilization and outcomes}

Patients will be classified into the study cohorts based on the index prescription during the index period (January 1, 1999 - December 31, 2001). The first prescription of a study medication (Risperidone, Olanzapine, Quetiapine or typical antipsychotics) during the index period will be defined as the index prescription. Intent to treat approach will be employed wherein the outcomes will be attributed to the index prescription irrespective of the medication pattern subsequent to index prescription. The affect of index antipsychotic on healthcare costs and various components of healthcare utilization such as physician visits, hospitalizations and length of stay, emergency room visits, and concomitant psychiatric prescription use will be evaluated. In addition, persistence with the index prescription and adherence to the index prescription will also be compared across the cohorts. Adherence will be measured in terms of medication possession ratio (MPR).

Multivariate regression analysis will be conducted with each of the outcome variable as dependent variable and antipsychotic type as independent variable. Various other factors that affect the outcome variables such as age, gender, prescribing physician specialty, schizophrenia/bipolar subtype, disease severity, schizophrenia/bipolar subtype, other mental illnesses, substance abuse, alcohol abuse, pre-index typical antipsychotic and other psychiatric medication use, pre-index health care costs and utilization will be controlled for in the multivariate analysis. As the assignment of patients into the index cohorts is not randomized, sample selection techniques will be 
used to control for sample selection bias. Figure 3 presents the conceptual framework describing the relationship between the index antipsychotic therapy and healthcare utilization and outcomes. 
Figure 3: Conceptual framework for Phase 2

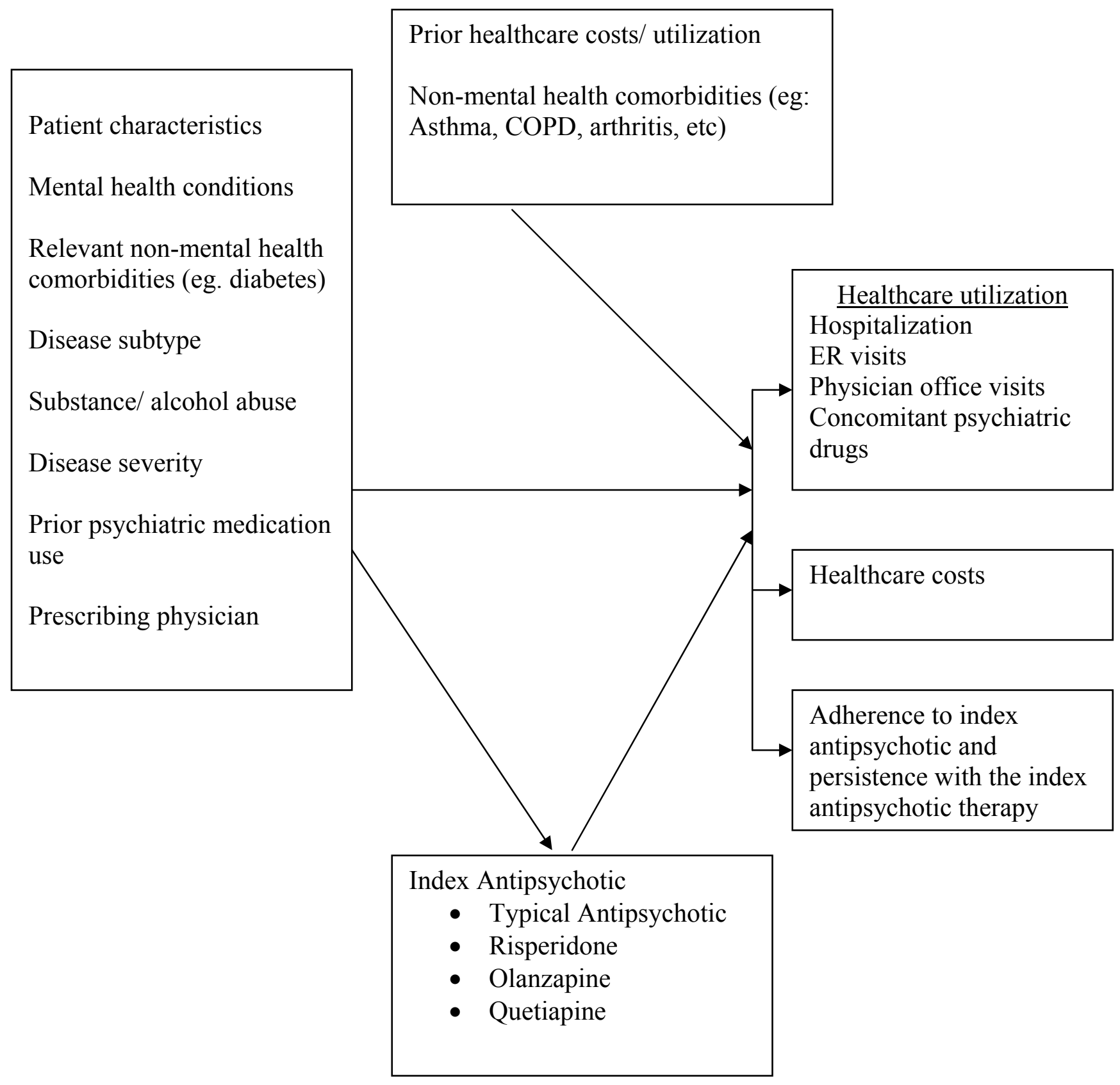




\section{Sample Selection Bias Adjustment}

As there is no randomization in studies based on claims database, they are prone to selection bias. Selection bias is a systematic error associated with how the study sample is derived from study population and/or how study subjects are assigned to treatment group (Strom, 2005). If we had all variables that are associated with the likelihood of receiving certain antipsychotic, we can control for them in a multivariate regression. However, information on many relevant variables usually is not available in retrospective data. This can bias the regression coefficients in a multivariate regression model estimating affect of treatment on costs and utilization. Unobserved variables could be clinical variables needed to determine patient's severity, prescribing physician's preferences and prior experience, patient preference, and market structure forces

Heckman two-stage technique is a specialized econometric technique to control for the sample selection bias (Greene, 2000; Heckman, 1979). The main concept behind the technique is that though we cannot correct the sample, we can correct the estimation. It tries to adjust the final estimation from the regression analysis for the unobserved or omitted variables.

Heckman two-stage technique involves the following two steps:

\section{Step 1:}

The first step of the Heckman two-stage technique involves developing a selection model. The selection bias is present due to differences in certain characteristics between the study cohorts that are related to the outcome variables (cost and utilization). The selection model compares the study cohorts to determine these differences. Heckman first-stage sample selection model uses a probit model as the selection model 
when there are two choices. Lee's modification of Heckman sample selection model involves use of a multinomial logit (MNL) model as the selection model when there are more than two choices (Greene, 2000).

In the selection model, the dependent variable is the choice of drug therapy (or any treatment group). The independent variables are relevant observed variables such as age, gender, race, comorbidities, physician specialty and prior medication history that can affect the choice of drug therapy. The affect of these observed variables on the choice of drug therapy is estimated using the selection model. When the affect of these observed variables on the dependent variable is removed, the remaining variation in the dependent variable is due to the unobserved variables. Therefore, the residuals of the selection model provide information on the unobserved variables and are used to calculate a covariate or a control factor that can correct for selection bias in the second-stage model. Step 2:

The second-stage model is a multivariate regression model where the dependent variable is the study outcome such as cost and utilization. The independent variables are relevant variables that can influence cost and utilization such as patient demographics, comorbidities, prior healthcare utilization, and choice of drug therapy. The independent variables in the second-stage regression equation may overlap with those in the first-stage regression equation. In addition, the control factor calculated in the first-stage selection model to control for unobserved variables is also included as an independent variable in the second-stage regression model.

If the coefficient of the control factor in the second-stage regression model is significant, it indicates that selection bias was present and some variation in the 
dependent variable is due to unobserved variables. However, the coefficient of the control factor captures the effects of unobserved variables on the outcome variables in the second-stage regression model. This results in unbiased coefficients for other independent variables in the model.

Heckman's two-stage model has been used successfully in many outcomes research studies (Crown et al., 1998; Crown, Treglia, Meneades, \& White, 2001; McCollam, Lage, \& Bala, 2001). Other methods such as propensity scores technique and instrumental variable can also be used to control for selection bias. However, propensity scores technique is more laborious and can reduce the sample size to matched observations. Also, it controls for only observed variables whereas Heckman two-stage technique controls for both observed and unobserved variables. The hindrance in using instrumental variable technique is to find an instrumental variable in claims database that is related to treatment choice but not to treatment outcomes (Crown et al., 1998)

\section{STUDY OBJECTIVES}

The overall goal of phase I is to study the pattern of antipsychotics use and its effects on utilization of healthcare services and expenditures. The goal of phase II is to compare risperidone, olanzapine, quetiapine and typical antipsychotics on multiple healthcare outcomes. Specific research questions necessary to achieve these goals are as follows: 


\section{Phase I}

\section{Research Question 1}

To determine the annual prevalence rate of schizophrenia and bipolar disorder in West Virginia Medicaid from 1998 to 2002

Rationale: This is an exploratory question to assess prevalence rate of schizophrenia and bipolar disorder in West Virginia Medicaid from 1998 to 2002

\section{Research Question 2}

To determine the medical conditions for which antipsychotics are being prescribed in the West Virginia Medicaid population as well as describe patterns of distribution of certain demographic factors such as age, gender, and ethnicity in patients using antipsychotics. Rationale: This is an exploratory question to assess prevalence of antipsychotic use for different medical conditions and differences in antipsychotic utilization by age, gender and ethnicity.

\section{Research Question 3}

For schizophrenia and bipolar disorder patients, determine different types of utilization pattern of antipsychotics.

Rationale: For schizophrenia and bipolar disorder patients, there is no difference in the patterns of antipsychotic utilization in terms of:

1) Polytherapy

2) Monotherapy
a) switching
b) interrupted therapy
c) continuous therapy 
3) Stabilization of index antipsychotic (for schizophrenia patients)

\section{Research Question 4}

For schizophrenia and bipolar disorder patients, determine the gaps between the refills of antipyschotics.

Rationale: There are no gaps in the antipsychotic therapy.

\section{Research Question 5}

For schizophrenia and bipolar disorder patients, determine predictors of different utilization patterns of antipsychotics

Null Hypothesis: There is no association between utilization patterns of antipsychotics and patient demographics, prescribing physician type, mental health diagnosis, other medical diagnosis, type of antipsychotic, year of index antipsychotic, pre-index concomitant medication use, pre-index alcohol and substance abuse and pre-index healthcare utilization.

\section{Research Question 6}

For schizophrenia and bipolar disorder patients, determine the relationship between utilization pattern of antipsychotics and total health-related healthcare costs. Null Hypothesis: There is no association between utilization patterns of antipsychotics and total health-related healthcare utilization and costs.

\section{Research Question 7}

For schizophrenia and bipolar disorder patients, determine the relationship between utilization pattern of antipsychotics and mental health care utilization and costs. Null Hypothesis: There is no association between utilization patterns of antipsychotics and mental health healthcare utilization and costs. 


\section{Phase II}

\section{Research Question 8}

For schizophrenia and bipolar disorder patients, to compare the impact of quetiapine with risperidone, olanzapine, and typical antipsychotics on total and mental healthcare costs Null Hypothesis: There is no association between the choice of index antipsychotic drug and total and mental healthcare costs.

\section{Research Question 9}

For schizophrenia and bipolar disorder patients, to compare the impact of quetiapine with risperidone, olanzapine, and typical antipsychotics on components of mental healthcare costs (costs associated with mental health-related inpatient, emergency room, outpatient and pharmacy services)

Null Hypothesis: There is no association between the choice of index antipsychotic drug and costs associated with mental health-related inpatient, emergency room, outpatient and pharmacy services

\section{Research Question 10}

For schizophrenia and bipolar patients, to compare the impact of quetiapine with risperidone, olanzapine, and typical antipsychotics on mental health-related healthcare hospitalizations

Null Hypothesis: There is no association between the choice of index antipsychotic drug and costs associated with mental health-related healthcare hospitalizations. 


\section{Research Question 11}

For schizophrenia patients and bipolar disorder patients, to compare the impact of quetiapine with risperidone, olanzapine, and typical antipsychotics on mental healthcarerelated emergency room visits

Null Hypothesis: There is no association between the choice of index antipsychotic drug and costs associated with mental healthcare-related emergency room visits.

\section{Research Question 12}

For schizophrenia and bipolar disorder patients, to compare the impact of quetiapine with risperidone, olanzapine, and typical antipsychotics on mental healthcare-related outpatient visits

Null Hypothesis: There is no association between the choice of index antipsychotic drug and costs associated with mental healthcare-related physician visits.

\section{Research Question 13}

For schizophrenia and bipolar disorder patients, to compare the impact of quetiapine with risperidone, olanzapine, and typical antipsychotics on psychiatric medication utilization Null Hypothesis: There is no association between the choice of index antipsychotic drug and costs associated with psychiatric medication utilization.

\section{Research Question 14}

For schizophrenia and bipolar disorder patients, to compare the impact of quetiapine with risperidone, olanzapine, and typical antipsychotics on patient's adherence to the index medications in the post-index period Null Hypothesis: There is no association between the choice of index antipsychotic drug and patient's adherence to the index medications in the post-index period. 


\section{Research Question 15}

For schizophrenia and bipolar disorder patients, to compare the impact of quetiapine with risperidone, olanzapine, and typical antipsychotics on subsequent index antipsychotic therapy modification

Null Hypothesis: There is no association between the choice of index antipsychotic drug and subsequent antipsychotic switching or discontinuation.

\section{STUDY SIGNIFICANCE}

The results of this study will describe the prevalent pattern of antipsychotic drug use in the WV Medicaid and report the costs and health services utilization associated with these patterns. This can give us a picture of complex pharmacotherapy regimens that are used to manage patients in clinical practice. It will help us understand both patient behavior such as medication adherence as well as prescriber behavior in the West Virginia Medicaid program. Economic endpoints will also be relevant from a formulary decision making perspective. It can identify the consequences of inappropriate prescribing such as polytherapy that can add to the already strained Medicaid budget covering expensive antipsychotics. This study can provide the basis for need for interventions such as academic detailing and other educational programs to improve prescribing patterns.

As newer atypicals are similar in efficacy, costs associated with their use can play an important role in treatment decisions. Considering the high acquisition costs of atypical antipsychotics, it important to monitor their utilization and costs within the 
system. In the proposed study, comparisons of antipsychotics will be differentiated in terms of multiple endpoints such as hospitalizations, emergency room visits, concomitant medication use, adherence and treatment discontinuation rates to give a better understanding of their impact on costs. The study will also control for selection bias to provide a better estimation of affect of choice of antipsychotics on costs and utilization. The results of this study will also be useful in understanding relatively less studied economics of antipsychotic use in bipolar disorder. These results can be useful to WV Medicaid to develop policies to provide better medication management for schizophrenia and bipolar patients.

\section{LIMITATIONS}

Use of administrative data can have certain disadvantages (Motheral et al., 1997). Inaccurate identification of diseases can occur due to errors in coding. Identification of cases in a research study solely on the basis of an outpatient visit can seriously affect the validity of study. However, hospital-based diagnoses have been shown to be very accurate (Bright, Avorn, \& Everitt, 1989). A study by Schwartz et al reported that about $90-94 \%$ of cases with psychiatric diagnoses in Medicaid matched with the diagnoses in the chart demonstrating a very high reliability (Schwartz, Perlman, Paris, Schmidt, \& Thornton, 1980). A study by Lurie et al validated using an inpatient claim or two outpatient claims with ICD codes for schizophrenia within past 2 years as inclusion criteria to accurately identify schizophrenics in Medicaid. They validated the diagnosis with the help of psychiatrics using DSM-III-R criteria for schizophrenia. Therefore, the inclusion criteria of an inpatient claim or two outpatient claims within past 2 years could 
be used with considerable confidence in our study to identify cases of schizophrenia in the WV Medicaid population (Lurie P, Popkin M, Dysken M, \& Finch M., 1992).

A claim for a prescription in the database indicates that the claim has been paid to the provider and not that medication has been taken correctly by the patient. Claims for a drug do not necessarily indicate adherence with the therapy. However, databases contain information such as date of service, amount dispensed and days of supply that can be used to calculate proxy measures for compliance. Several studies have found significant correlations between adherence measured from refill information and other methods such as self-report, pill count, and medication diary (Choo et al., 1999).

Threats to external validity are relatively lower in database studies as compared to randomized clinical trial. However, one should be careful in claiming generalizability as characteristic of study population in different databases may differ according to age, sex, race, socioeconomic status, geography, etc. Also, various factors such as co-pays, formularies, and provider access may be unique to West Virginia Medicaid. Practice patterns and costs also vary across time and geographically. Thus, findings from a study population of a certain plan may not apply to populations from other plans. Descriptive information about our study population characteristics will be given so that the readers can weigh the similarities and differences between the study population and the population in question.

Due to lack of random assignment, there may be differences between patients initiated on different therapies. Therefore, adjustments for sample selection bias are necessary. Risk adjustments are also important to control for differences between the 
groups. A study by Sernyak et al validated use of sociodemographic variables, prior utilization and cost variables obtained from administrative data as risk adjusters to predict future utilization and costs. The study compared models that used administrative data alone with models that used administrative data as well as clinical data from a prospective, double-blind clinical trial. The models using combination data yielded an adjusted r-square of 0.31-0.27 that was not significantly different from adjusted r-square of $0.24-0.28$ that was obtained from models that used only administrative data (Sernyak \& Rosenheck, 2003). Our study will control for confounding using sociodemographics, pre-index utilization and costs as well as other variables as covariates. 


\section{CHAPTER TWO}

\section{LITERATURE REVIEW}

\section{Schizophrenia}

\section{Epidemiology and Cost}

Schizophrenia is a complex psychiatric disorder affecting about $0.2 \%$ to $2 \%$ of the population. Despite the low prevalence of the disease, schizophrenia is the most expensive psychiatry disorder to treat (Knapp, 1997). The burden of disease is high due to early onset, chronic nature, hospitalizations, comorbidities and disabilities. Schizophrenia costs constitute $2.5 \%$ of total healthcare expenditures. Schizophrenic patients consume about $10 \%$ of social security benefits. About $70-80 \%$ schizophrenic patients are unemployed (Foster \& Goa, 1998). In 1991, direct and indirect costs associated with schizophrenia were estimated to be $\$ 18.6$ billion and $\$ 46.5$ billion, respectively. Hospitalizations are responsible for almost $70 \%$ of costs, drug costs for almost $10 \%$, and outpatient costs usually make up the rest of the $20 \%$ (Revicki, 1997). Prolonged / frequent episodes, long term hospitalization, and care in intensive community programs incur most of the direct costs. Cost of social welfare, criminal justice services and family productivity are included in the indirect costs (Revicki, 1997). Annual total healthcare costs per schizophrenia patient was estimated to range between $\$ 16,000$ and \$57,000 in 1999 (Mauskopf et al., 1999).

Schizophrenia symptoms usually appear in the late teens or early twenties in men and in the twenties to early thirties in women (APA, 1997; Crismon ML \& Dorson PG., 1997). Prevalence is similar between the genders (APA, 1997; Crismon ML et al., 1997). The disease is chronic in nature and relapses requiring hospitalization could be frequent. 
Most patients are readmitted within 3 to 5 years (APA, 1997). Risk of other medical illnesses and mortality from suicide is also high among patients with Schizophrenia (Carpenter, Jr. \& Buchanan, 1994). Schizophrenic patients are at risk for substance abuse, suicide, and disability (APA, 1997). Almost 25-50\% attempt suicides and almost $10 \%$ commit suicide. About $10 \%$ of disabled US population is schizophrenic (Foster et al., 1998).

\section{Disease Description}

Symptoms of schizophrenia include thought disorders, emotional changes, delusion and perception disorders (hallucinations, sensory effects), aggressive behavior, impaired judgment, ideas of reference, withdrawal from external reality, eccentric or ritualistic behavior (Crismon ML et al., 1997). A schizophrenic patient may not be able to function in society and take care of himself (Crismon ML et al., 1997). According to the American Psychiatry Association (APA) guidelines, (APA, 1997) phases of schizophrenia can be classified into: 1) Acute phase: The patient exhibits some of the most severe symptoms in this phase. The patient may experience hallucinations, delusions, and severely disorganized behavior and/or thinking. Prior to this phase, the individual may become more withdrawn or behave in an unusual manner. Symptoms may gradually become more severe and lead to an acute episode. An acute episode usually requires hospitalization. The duration of this phase depends on several factors including patient characteristics and environment. 2) Stabilization phase: This phase begins as the psychotic symptoms decrease in severity and may last for 6 months or more. 3) Stable phase: Most of the psychotic symptoms become stable in this phase. 
Some idiosyncratic behaviors and nonpsychotic symptoms, such as depression, anxiety, tension, or insomnia, may still persist.

\section{Pharmacotherapy in Schizophrenia}

Schizophrenia treatment comprises of pharmacotherapy and psychosocial treatment or rehabilitation (Lehman et al., 2004b). Pharmacotherapy of schizophrenia involves use of conventional antipsychotics and relatively newer atypical antipsychotics. The typical or conventional antipsychotics include the phenothiazines (chlorpromazine, fluphenazine, mesoridazine, perphenazine, prochlorperazine, trifluoperazine, and thioridazine), butyrophenones (haloperidol), thioxanthenes (thiothixene), and dihydrolones (molindone and loxapine). Efficacy of the typical agents is due to blockade of the dopamine $\mathrm{D}_{2}$ receptors. The blockade also is responsible for their side-effect profiles. Most of the conventional antipsychotics are comparable in efficacy. Typically, these agents decrease only the positive symptoms of schizophrenia. Depending on the extent of blockade, these agents may actually worsen negative symptoms. The common side-affects of typical antipsychotics (Crismon ML et al., 1997) are:

a. Acute extrapyramidal effects: These can lead to acute dystonia (spasm of face, neck, back muscles), Akathesia, Parkinsonism (bradykinesia, rigidity, tremor, mask face). b. Neuroleptic malignant syndrome: These are rare but can be fatal. These are characterized by course tremor, catatonia, fluctuating vital signs, metabolic abnormalities.

c. Chronic extrapyramidal effect or tardive dyskinesia can develop during long-term treatment. 
d. Autonomic effects such as antimuscarinic effects (dry mouth, constipation, difficulty urinating) or effects due to blockade of $\alpha 1$ receptors (orthostatic hypotension, sexual dysfunction).

e. Endocrine effects are responsible for hyperprolactinemia (due to dopamine receptor blockade) that can lead to galactorrhoea, amenorrhoea and gynaecomastia. Weight gain is possible due to hyperprolactinemia and 5HT blockade.

f. Psychological effects such as sedation and cognitive impairment can occur depending upon the drug.

\section{Atypical Antipsychotics}

Several atypical antipsychotics, especially effective in addressing both the negative symptoms and positive symptoms of schizophrenia, have entered the market in the last decade. For example, Clozapine (Novartis 1990), Risperidone (Janssen 1993), Olanzapine (EliLilly 1996), Quetiapine (Zeneca 1997), Ziprasidone (Pfizer 2001) and Aripiprazole (Bristol Myers Squibb 2002). These agents antagonize serotonin $5 \mathrm{HT}_{2 \mathrm{a}}$ and dopamine $\mathrm{D}_{2}$ receptors. Therefore, there is a lower incidence of extrapyramidal side effects (Carpenter, Jr. et al., 1994). These atypical antipsychotics have high acquisition costs but are more affective in controlling positive symptoms and have more tolerable extra pyramidal symptoms profile.

Efficacy of atypical antipsychotics is comparable to typical antipsychotics for most aspects of schizophrenia (Carpenter, Jr. et al., 1994; Carpenter, Jr. et al., 1994). Atypicals have similar or slightly better global clinical efficacy for positive symptoms as compared to typical antipsychotics. They have shown better relapse prevention than typical antipsychotics in controlled trials. Clozapine, risperidone, olanzapine and 
ziprasidone have been shown to be much better efficacy for negative symptoms as compared to typical antipsychotics. Quetiapine is similar to typical antipsychotic for negative symptoms. Atypical antisychotics show better efficacy than typical antipsychotics in controlling affective symptoms, depression and suicidality. Clozapine is much superior to typical antipsychotics and other atypical antipsychotics for treatment refractory patients (Carpenter, Jr. et al., 1994).

Side effect profile of atypical antipsychotics is much better than typical antipsychotics (APA, 1997; Carpenter, Jr. et al., 1994). Acute extrapyramidal effects $(E P S)$ are less likely with atypical antipsychotics. Neuroleptic malignant syndrome is rare but can occur. Chronic extrapyramidal effect / tardive dyskinesia is much less likely with atypical antipsychotics. Autonomic effects depend upon the drug but are similar to typical antipsychotics. Endocrine effects such as hyperprolactinemia are less likely with typical antipsychotics. However, recent studies have shown that risk of hyperglycemia, diabetes, and hyperlipedemia may be higher in patients using antipsychotics (Koro et al., 2002; Lindenmayer et al., 2003). These effects may vary from drug to drug (Koro et al., 2002; Lindenmayer et al., 2003). Also, weight gain can be greater due to 5HT and histamine receptor blockade. Psychological effects such as sedation depend on drug but are similar to typicals. Impaired cognition may be less than with typical antipsychotics. Clozapine can cause blood dyscrasias (agranulocytosis) and requires white count check frequently (Amadio, Cross, \& Amadio, Jr., 1997). 


\section{Antipsychotic Treatment Guidelines for Schizophrenia}

The Texas Medication Algorithm Project (TMAP) has developed stepwise algorithms for schizophrenia pharmacotherapy. First published in 1999, they were updated in 2003 to include recommendations for ziprasidone and ariprazole (Miller et al., 1999; Miller, 2004). TMAP guidelines recommend using atypical antipsychotics (olanzapine/ quetiapine/ risperidone) as first-line of treatment (Miller et al., 1999). In 2003, ziprasidone and ariprazole were also included among the list of atypical antipsychotic to be used as first-line therapy (Miller, 2004). According to TMAP guidelines, duration of trial with the antipsychotic should be at least 3 weeks and at the most 10 weeks. The guidelines suggest trying the 3 atypicals in sequential order and then a typical antipsychotic before using clozapine. Monotherapy is recommended until clozapine fails. Weekly (or at least every two weeks) visits until the patient is stable is advised for outpatients who are switching antipsychotic medications. Other guidelines such as American Psychiatric Association (APA) guidelines suggest typical or atypical antipsychotics as first-line treatment whereas TMAP guidelines emphasize use of atypicals (American Psychiatric Association, 1997). However, APA guidelines do not recommend any form of polytherapy. TMAP guideline recommends polytherapy but only after a trial with clozapine fails.

\section{Antipsychotics Utilization Patterns in Schizophrenia}

Antipsychotic utilization patterns among schizophrenia patients in clinical practice can be classified into predominant categories such as polypharmacy, switching, and discontinuation. 


\section{Antipsychotic Polypharmacy}

Despite the lack of controlled clinical trials evaluating efficacy of antipsychotic polypharmacy, it has considerable prevalence in clinical practice. Prevalence of antipsychotic polypharmacy has been reported to range from $6.8 \%$ to $41 \%$ in various populations. An analysis of outpatient prescriptions in Veteran Administration (VA) schizophrenia population revealed only $6.8 \%$ received polytherapy (Leslie \& Rosenheck, 2001). Studies evaluating antipsychotic use among hospitalized patients have found higher rates $(16 \%$ - 45\%) of polytherapy (Schumacher, Makela, \& Griffin, 2003; Procyshyn et al., 2001; Procyshyn et al., 2004; McCue et al., 2003). Antipsychotic polypharmacy increased from $5.7 \%$ in 1995 to $24.5 \%$ in 1999 among the New Hampshire Medicaid beneficiaries with schizophrenia (Clark et al., 2002). Prevalence of antipsychotic polypharmacy between 1998 and 2000 was about $40 \%$ in a sample of Georgia Medicaid and California Medicaid Schizophrenia patients (Ganguly, Kotzan, Miller, Kennedy, \& Martin, 2004). Prevalence of antipsychotic polytherapy among Rhode Island Medicaid's elderly and disabled population was about 10\% in 2003 (Kogut, Yam, \& Dufresne, 2005). A retrospective review of medication information for all patients discharged from a tertiary care psychiatric facility between January 1, 2000 and December 31, 2000 revealed highest rate of antipsychotic polypharmacy among patients with schizoaffective disorder (49.3\%), followed by schizophrenia (44.7\%) (Procyshyn et al., 2004)

As each atypical antipsychotic has a unique receptor profile, there may be theoretical rationale in combining these medications to manage symptoms for some patients. Physician surveys have revealed that the most common reason cited for 
prescribing polytherapy was to reduce the positive symptoms. In many cases, physicians have stated that addition of another antipsychotic was intended to be transitional but continued because of patient's preference or improvement in symptoms (Sernyak \& Rosenheck, 2004; Tapp et al., 2003). A physician survey by Schumacher and colleagues reported therapy augmentation as the most common rationale for polytherapy. However, majority of the patients treated by these physicians had not received a trial of atypical or typical monotherapy or clozapine before receiving polytherapy (Schumacher et al., 2003). Case reports and retrospective charts reviews evaluating antipsychotic polypharmacy do not provide any conclusive information regarding efficacy and adverse effects of this practice (Freudenreich \& Goff, 2002). One open prospective trial reported increased risk of mortality during 10-year follow-up period among schizophrenia patients on antipsychotic polytherapy (Waddington, Youssef, \& Kinsella, 1998). The difference in mortality rates may be due to selective prescription of antipsychotic polypharmacy to more severe patients.

There are very few studies evaluating economic consequences of antipsychotic polytherapy. Majority of these studies are restricted to inpatient settings. McCue and colleagues used hospital records of an inpatient psychiatry facility to compare schizophrenia patients who were discharged in the year 1995 to schizophrenia patients who were discharged in 2000. They reported that no patients discharged in 1995 received polytherapy whereas $15.9 \%$ of patients discharged in 2000 received polytherapy. They also report lesser adverse drug reactions and shorter length of hospital stay among patients discharged in 2000. As they examined cross-sectional data in 1995 and 2000, we cannot make inference regarding association of polypharmacy with decreased adverse 
drug reactions and length of stay. Prescribing practices for schizophrenia has changed considerably between 1995 and 2000 due to greater use of atypical antipsychotics. Fewer adverse effects may be attributed to fewer EPS side-effects with the atypicals. A retrospective review of medications prescribed during discharge from a tertiary psychiatry facility to non-elderly patients between November 1, 1996 and October 31, 1998 revealed that $27.5 \%$ were discharged on antipsychotic polypharmacy. Despite a small sample size of the study $(\mathrm{N}=229)$, antipsychotic polytherapy was significantly associated with greater use of anticholinergics compared to monotherapy (Procyshyn et al., 2001). Centorrino and colleagues used a case-control study design to compare polypharmacy with monotherapy using inpatient records of psychiatric patients. Seventy patients receiving 2 or more antipsychotics for at least 3 consecutive days were identified as cases and matched with 70 patients who received only one antipsychotic. Cases and controls were matched on gender, age, psychiatric disorder (schizophrenia/ bipolar disorder/ or other disorders) and illness severity. The analysis revealed that although there were similar clinical improvements in both groups, patients on polytherapy had $55 \%$ longer length of stay and $56 \%$ higher risk of adverse effects than patients on monotherapy (Centorrino et al., 2004).

\section{Antipsychotic Switching and Discontinuation}

Pharmacotherapy of schizophrenia often requires switching between the medications (Bogan, Shellhorn, Brown, McDanald, \& Suppes, 2000; Burns, Chabannes, \& Demyttenaere, 2002; Menzin, Boulanger, Friedman, Mackell, \& Lloyd, 2003). Switching can occur if there is inadequate control of positive symptoms, residual negative symptoms such as apathy and mood symptoms. Atypical antipychotics are 
better at controlling mood symptoms like depression. Atypicals also have fewer pyramidal side-effects compared to conventional antipsychotics. Therefore, many patients can be switched to atypicals. Introduction of new atypical drugs in market can lead to switches from previously used drugs. Prescriber may switch drug if the patient relapses despite compliance. However, weight gain and high cost of atypical drugs can also lead to non-compliance(1999).

Non-compliance is a critical problem with antipsychotic therapy. Noncompliance is mainly a result of disabling adverse effects such as extrapyramidal symptoms (EPS) that can offset the benefits of the therapy (Van Putten, 1974). EPS symptoms include muscular rigidity and cramping, tremors, gait disturbances, drooling, agitation, and involuntary abnormal movements of the lips, tongue, and facial muscles. Other adverse effects such as gynecomastia, galactorrhea, amenorrhea and impotence that are related to elevated prolactin concentrations also reduce compliance (Hamner, Arvanitis, Miller, Link, \& Hong, 1996). About 50\% of schizophrenic patients who discontinue their antipsychotic therapy relapse within a year (Pigott et al., 2003). Noncompliance is also responsible for repeated hospitalizations also known as "revolving door syndrome" among schizophrenics (Haywood et al., 1995). About 37\% of direct costs within 2 years of discharge after first hospitalization results due to non-compliance (Weiden \& Olfson, 1995). Because of better side-effect profile, atypical antipyschotics have shown better compliance and are recommended as the first-line treatment for schizophrenia. About $30 \%$ of patients relapse with typical antipychotics whereas about $20 \%$ of patients relapse with atypical antipsychotics (Weiden, Aquila, \& Standard, 1996). 
Patient non-compliance due to disease nature and side-effects of medications can result in high discontinuation rates. Increasing patient persistence with the given antipsychotic therapy is a challenge for health care providers. In addition, about 5\%-25\% of schizophrenia patients are treatment refractory (Brenner et al., 1990). The physician's attempts to control symptoms in these patients can lead to varying patterns of antipsychotic use.

In 1995, Williams and colleagues studied use of antipsychotic in an outpatient patient population with schizophrenia using Regenstrief Medical Record System. Out of 316 study sample, about $88 \%$ received typical and $12 \%$ received atypical antipsychotic. About $71.5 \%$ of patients received only antipsychotic whereas about $25 \%$ of patients switched to another antipsychotic during the 1-year follow-up period. About $30 \%$ of patients received continuous antipsychotic treatment for one year. About $67.1 \%$ of patients had a gap of 1-11 months in their antipsychotic therapy. Factors associated with continuous use of antipsychotics in this patient population were not investigated (Williams et al., 1999). A study by Al-Zakwani and colleagues followed 469 managed care plan enrollees who were initiated on antipsychotics irrespective of mental health diagnosis for one year. Patients were initiated on antipsychotic between 1999 and 2000. About $81.8 \%$ received atypical and $18.2 \%$ received typical antipsychotic. The proportion of patients who switched from atypical antipsychotics (18.2\%) was not significantly different from proportion of patients who switched from typical antipsychotics (12.9\%) (Al Zakwani et al., 2003).

There are two studies investigating various types of antipsychotic utilization patterns in schizophrenia patient population predominantly on typical antipsychotics. 
McCombs and colleagues used administrative claims data from the California Medicaid program (Medi-Cal) to study utilization pattern of antipsychotics from 1987 to 1996. A total of 2,655 schizophrenia patients were identified between 1987 and 1996. During the time period of the study, they found that about $98 \%$ of patients were on conventional antipyschotics. About $47 \%$ of patients switched or augmented their therapy within one year. Only $11.6 \%$ of patients received continuous antipsychotic therapy for one year. The average duration of antipsychotic therapy was 142 days. They also reported that factors such as increasing age, manic disorder, major depression, bipolar disorder and nonorganic psychosis are associated with switching (McCombs et al., 1999b). A cost analysis of association between these patterns of use and healthcare costs revealed that antipsychotic switching can increase the annual total cost by $\$ 9,719$ (McCombs et al., 2000). However, as $98 \%$ of their patient population was on typical antipsychotics, these factors do not provide information on switching from atypical therapy.

Loosbrook and colleagues used an employer claims database to study utilization pattern of antipsychotics and associated costs with utilization patterns during the year 1997 (Loosbrock, Zhao, Johnstone, \& Morris, 2003). The study reports about 26\% of schizophrenia patients received no antipsychotic therapy, $52 \%$ received only one antipsychotic, $13 \%$ switched, $7 \%$ augmented with additional antipsychotic, and $2 \%$ received more than one antipsychotics at the beginning of the year. Regression analysis, adjusting for various covariates such as age, gender, schizophrenia subtype, comorbidities, and prior costs, revealed that antipsychotic switching and augmentation can increase the total cost by $\$ 4,706$ and $\$ 4,244$, respectively. 
None of the above studies adjusted for selection bias that may exist among patients who follow different patterns of utilization. Majority of antipsychotic use was of typicals as the most recent data used was upto 1997.

\section{Bipolar Disorder}

Epidemiology and Cost

Bipolar disorder, also referred to as manic depressive illness, is characterized by periodic episodes ranging from mania (e.g. euphoria, grandiosity, impulsivity, excessive libido) to the depression (e.g. depression, anxiety, violence, suicidal thoughts and activity). Psychotic symptoms such as delusions and hallucinations are also common in case of acute mania (Perry, Tarrier, Morriss, McCarthy, \& Limb, 1999; APA, 1994). The lifetime prevalence of bipolar disorder in the US population is about $1.2 \%$ to $1.6 \%$ (Kessler et al., 1994). The estimated economic burden of the disorder is about $\$ 45$ billion. The direct costs are estimated at $\$ 7.6$ billion and indirect costs at $\$ 38$ billion. About $60-70 \%$ of direct costs were attributable to inpatient costs (Wyatt et al., 1995). Medication costs constitute about $2 \%$ of direct costs. The annual medication cost per treated patient was about $\$ 548$ (Wyatt et al., 1995). About 75\% of bipolar patients undergo at least one hospitalization during the course of their illness (Lish et al., 1994). About $10 \%$ of inpatient healthcare in US is utilized by bipolar patients (Narrow et al., 1993). Costs for patients with bipolar disorder is higher than costs for patients treated for depression or diabetes (Simon et al., 1999). Also, the disorder is the sixth leading cause of disability in the world (Jenkins, 1997). The chronic relapsing nature of the bipolar disorder impairs psychosocial functioning and ability to work in patients (Goldberg, Harrow, \& Grossman, 1995). About $50 \%$ of patients are unable to live independently or 
retain employment (Levine, Chengappa, Brar, Gershon, \& Kupfer, 2001). Among employed bipolar patients, the employment does not match the educational level attained by these patients before the onset of the disease (Tse \& Walsh, 2001).

About $20 \%$ of severely mentally ill patients have diagnosis of bipolar (Clark et al., 1998). The prevalence of bipolar disorder is similar among adolescents and adults and between genders (Lewinsohn, Klein, \& Seeley, 1995; Weissman et al., 1996). Mean age of onset is 18 years for bipolar I and 22 years for bipolar II (Weissman et al., 1988). The rate of mortality among untreated bipolar disorder patients is about $19 \%$ and is comparable to heart disease and cancer patients (Hopkins \& Gelenberg, 1994). Suicide rates are about 11-15\% (Hilty, Brady, \& Hales, 1999; Shepherd \& Hill, 1996). Commonly prevalent comorbidities among bipolar patients are alcohol and drug abuse, anxiety and personality disorders (Shepherd et al., 1996; Strakowski et al., 1998; Strakowski \& DelBello, 2000).

\section{Disease Description}

The Diagnostic and Statistical Manual of Mental Disorders, Fourth Edition (DSMIV) classifies bipolar disorder into: bipolar I, bipolar II and cyclothymia (APA, 1994). Bipolar I disorder is characterized by manic-depressive episodes of duration greater than 1 week. Manic episode is characterized by feelings of self-importance, elation, talkativeness, increased sociability, irritability, impatience, hyperactivity, and a decreased need for sleep. Mixed states consisting of manic or hypomanic (milder form of mania) episodes and depressive episodes can also occur in bipolar I patients. Bipolar II disorder is characterized by major depressive episodes along with episodes of hypomania. Patients with bipolar depression show retarded mental and physical processes, extreme 
fatigue, and hypersomnia. Bipolar depression may be difficult to distinguish from a unipolar major depressive episode. Cyclothymia refers to less severe but multiple episodes of hypomania and depression. DSM also defines a phenomenon of rapid cycling in bipolar episodes where manic and depressive episodes alternate at least 4 times in 12 months. Studies have reported about $34-61 \%$ relapse rates among patients receiving treatment (Bowden, 2000; Harrow, Goldberg, Grossman, \& Meltzer, 1990; O'Connell, Mayo, Flatow, Cuthbertson, \& O'Brien, 1991).

\section{Pharmacotherapy in Bipolar Disorder}

Treatment for bipolar disorder aims to stabilize mood and alleviate mania or depression. Although mood stabilizer such as lithium has been recommended as the firstline treatment for bipolar disorder, about $42-62 \%$ of patients are resistant to lithium (Solomon, Keitner, Miller, Shea, \& Keller, 1995). About $80 \%$ of patients with mixed and rapid cycling bipolar disorder are resistant to lithium (Tohen \& Zarate, Jr., 1998). There are problems with lithium treatment like its toxic doses are close to its therapeutic range. Common side-effects of lithium are tremor, gastrointestinal irritation, cognitive dulling, and renal, thyroid and cardiovascular side-effects on prolonged use. Early discontinuation of lithium therapy is common (Moller \& Nasrallah, 2003). Other medications recommended for bipolar treatment include anticonvulsants, antipsychotics, and antidepressants. Anticonvulsants such as divalproex and carbamazepine are used for acute treatment and long term maintenance therapy (Moller et al., 2003).

Antidepressants are recommended as an adjunct with mood stabilizer in severely depressed and suicidal patients (Bottlender, Rudolf, Strauss, \& Moller, 2001). Use of antidepressants is risky as they may induce mania or rapid cycling (Moller et al., 2003). 
Antipsychotics have been recommended as an adjunct to a mood stabilizer in US for bipolar disorder with resistant mania, psychoses, agitation and insomnia (Sachs, Printz, Kahn, Carpenter, \& Docherty, 2000). However, monotherapy with antipsychotics has been used as first-line treatment for severe and hospitalized bipolar patients in Europe (Moller et al., 2003). Typical antipsychotics show similar efficacy to lithium for treating mania but may fail to prevent or may induce depression (McElroy, Keck, Jr., \& Strakowski, 1996). Also, the risk of tardive dyskinesia with typical antipsychotics can lead to non-compliance and relapse (Moller et al., 2003). Recent studies have shown efficacy of atypical antipsychotics in treating both manic and depressive symptoms in bipolar patients with and without psychoses (Frye et al., 1998; Sanger et al., 2003;

Shattell et al., 2004; Strakowski, Del Bello, Adler, \& Keck, Jr., 2003). Use of olanzapine was recommended as an alternative to lithium or divalproex for acute mania by Texas Medication Algorithm (Suppes et al., 2003). Though Olanzapine, quetiapine, risperidone, ziprasidone and ariprazole were recently approved by FDA for use in bipolar disorder (Shattell et al., 2004; Perlis, 2005), the use of antipsychotics to treat mania has been widely prevalent in clinical practice (Licht et al., 1994).

\section{Treatment Guidelines for Bipolar Disorder}

Treatment guidelines for bipolar disorder differ between different committees such as TMAP, APA or expert consensus guidelines and according to bipolar subtype. In general, monotherapy with lithium, divalproex sodium or atypical antipsychotic is recommended as first-line treatment for mania or hypomania. In case of partial response or non-response, combination of mood stabilizer or anticonvulsant or atypical antipsychotic is usually advised (Fountoulakis et al., 2005). TMAP guidelines 
recommend monotherapy with lithium or divalproex or atypical antipsychotic as first-line treatment. APA guidelines recommend use of lithium or a combination of mood stabilizer with an atypical antipsychotic as the first-line therapy. For bipolar depression with psychosis, atypical antipsychotic is required as an alternative to electroconvulsive therapy (ECT) (Perlis, 2005).

\section{Antipsychotic Utilization Pattern in Bipolar Disorder}

There are very few studies investigating pattern of use of antipsychotics among bipolar patients. However, majority of studies looking at pharmacotherapy patterns in bipolar disorder have reported considerable prevalence of antipsychotic use as monotherapy and in combination with mood stabilizers among bipolar patients.

A meta-analysis of 16 studies published between 1980 and 1999 showed that use of typical antipsychotic was common in bipolar patients. About $84.7 \%$ of bipolar patients received typical antipsychotics. About $53.8 \%$ of typical antipsychotic use was as monotherapy whereas about $47.4 \%$ of typical antipsychotic use was in combination with a mood stabilizer (Tohen et al., 2001). A study by Russo and colleagues studied pattern of pharmacotherapy in bipolar disorder between 1994 and 1998 using a privately insured managed care patients. The study reports that out of 3,057 bipolar patients, $78 \%$ were using lithium or valproate, $16.4 \%$ were using typical antipsychotics, $12.4 \%$ were using atypical antipsychotics and 74\% were using antidepressants. As the drug categories were not mutually exclusive, patients could be on all or any of these medications. Patients who initiated on lithium or valproate, antipsychotics, antidepressants or anticonvulsants were followed for one year to study patterns of continuous or discontinuous use, and switch. However, results of logistic regression analysis showed that patients initiated on 
antipsychotics were more likely to use them for 12 months or more compared to patients initiated on lithium/valproate. As compared to lithium/valproate, patients initiated on antidepressants were more likely to switch and patients initiated on anticonvulsants were more likely to discontinue medications. Proportion of patients initiated on each drug category was not reported (Russo et al., 2002).

Craig and colleagues examined patterns of medication use among first-admission bipolar disorder patients between 1989 and 1995. A sample of 155 patients was followed for 24 months. Medication use and clinical assessments were observed at baseline, 6month and 24-month time period. Early and regular use of lithium/valproate or carbamazepine was associated with better Global Assessment of Functioning (GAF) scores and lesser time in remission. The study reported that antipsychotic use was associated with poor clinical outcomes in these patients (Craig et al., 2004). It is possible that more severe bipolar patients may be receiving antipsychotics. Also, most patients in the study received typical antipsychotics

Procyshyn and colleagues conducted a retrospective review of medication information for all patients discharged from a tertiary care psychiatric facility between January 1, 2000 and December 31, 2000. They found a 29.9\% prevalence of antipsychotic polypharmacy among bipolar disorder (Procyshyn et al., 2004).

\section{Antipsychotic Use for Other Indications}

Few studies have reported us of antipsychotics for other indications. Liperotti and colleagues used a cross-sectional data between January 1, 1999 and January 31, 2000 from 1,732 nursing homes from 5 US states. They reported that atypical antipsychotic use was associated with Parkinson's disease, depression, Alzheimer's disease, and non- 
Alzheimer dementia (Liperoti et al., 2003). Cooper and colleagues used Tennessee's Medicaid managed care data to study the use of antipsychotics among patients aged 2 to 18 years (Cooper et al., 2004). They report that antipsychotic use among children has increased almost 2-5 folds in attention-deficit/hyperactivity disorder, and affective disorders between 1996 and 2001. Other than schizophrenia, antipsychotics were also used in acute psychotic reaction and autism. A retrospective chart review of 2000 charts to determine atypical antipsychotic use as augmentation agent to antidepressants in treatment-resistant major depression patients captured 49 cases (Barbee, Conrad, \& Jamhour, 2004).

Antipsychotics use for conditions other than schizophrenia and bipolar disorders is still under investigation in clinical trials and is not approved by FDA (Shelton, 2003; Ostroff \& Nelson, 1999; Kaplan, 2000). However, prevalence of antipsychotic use in other conditions is present in clinical practice and needs to be documented.

\section{Antipsychotic Therapy and Economic Outcomes in Schizophrenia}

Pharmacoeconomic studies in schizophrenia focus on testing the hypothesis that atypical antipsychotics will decrease utilization of healthcare services leading to reduction in overall treatment costs and improve patient outcomes. Most pharmacoeconomic evaluations focus on comparing total healthcare costs, which are definitely an important endpoint from formulary decision-making perspective.

Atypical antipsychotics have been shown to offset inpatient and outpatient costs as compared to typical antipsychotics. However, they are also responsible for considerable proportion of pharmacy or formulary costs (Csernansky et al., 2002; 
Gianfrancesco et al., 2002; Gibson et al., 2004; Nightengale et al., 1998). The challenge for healthcare policymakers is to determine the degree to which benefits of antipsychotic overweigh their high acquisition costs. Pharmacoeconomic comparisons between atypical antipsychotics have reported varying results depending upon study population, outcome measures, research and statistical design. Most of the comparisons are between risperidone and olanzapine and have total treatment costs or inpatient drug costs as outcome measure.

A retrospective database study using an employer claims database from 1994 to 1999 compared one-year treatment outcomes between olanzapine and risperidone (Zhao, 2004). The outcomes measures were duration of therapy and direct healthcare costs. Results of multiple regression analysis show that the patients initiated on olanzapine ( $\mathrm{n}=$ 142) had significantly lower costs compared to patients initiated on risperidone $(\mathrm{n}=431)$. The average duration of therapy was also longer with olanzapine compared to risperidone. Another study by Rascati and colleagues also reported that average duration of therapy was longer with olanzapine compared to risperidone based on analysis of Texas Medicaid data from 1996 to 1999. However, risperidone has lower total medical costs compared to olanzapine (Rascati et al., 2003). Better medication adherence for olanzapine was also reported by Gibson and colleagues in their analysis of Michigan Medicaid data from 1995 to 1998 . Olanzapine cohort had about $60 \%$ adherence compared to risperidone cohort with $54 \%$ adherence. Though there were no significant differences in total healthcare costs across the cohorts, exclusion of index medication costs resulted in significantly lower total costs for olanzapine cohort compared to risperidone (Gibson et al., 2004). The differences in pharmacy costs may be attributed to 
higher acquisition costs of olanzapine (Procyshyn \& Zerjav, 1998; Byerly et al., 2003; Voris \& Glazer, 1999) or better adherence resulting greater utilization of index drug. A prospective observational study comparing olanzapine and risperidone showed that olanzapine had lesser psychiatric hospitalization than risperidone-treated schizophrenia patients within one year period (Ascher-Svanum et al., 2004).

There are other studies that report cost savings with risperidone, especially for inpatient drug costs. The Risperidone Olanzapine Drug Outcomes studies in Schizophrenia (RODOS) used retrospective chart reviews of hospitalized patients $(\mathrm{N}=$ 1,901) from nine countries. The outcomes measures were utilization and costs of inpatient drugs among patients using olanzapine $(n=977)$ or risperidone $(n=924)$. The average total inpatient drug costs were greater for olanzapine compared to risperidone (\$297 vs. \$159). This difference was attributed to longer duration of use for olanzapine compared to risperidone. However, after adjusting for baseline difference and comparing the costs on daily basis, olanzapine group still showed significantly higher inpatient costs compared to risperidone (Kasper et al., 2001). Similar results regarding inpatient drug costs were also reported by Kelly and colleagues in their study examining inpatient data of 789 patients with schizophrenia or schizoaffective disorder initiated on risperidone or olanzapine between July 1997 and June 1998. Daily drug costs was lower among risperidone treated patients (\$6.42) compared to olanzapine patients $(\$ 12.29)$. In addition, they reported higher discharge rate after 30 days of treatment among risperidone patients (45\%) compared to olanzapine patients (32\%) (Kelly et al., 2001).

Another study evaluating antipsychotic costs, psychiatric inpatient and outpatient costs among schizophrenia patients initiated on risperidone and olanzapine concluded 
that though there are no significant differences in inpatient and outpatient costs among patients initiated on risperidone or olanzapine, it is more economic to prescribe risperidone due to its lower drug acquisition costs (Byerly et al., 2003). The study was conducted using VA data among hospitalized patients who were initiated on risperidone $(n=23)$ or olanzapine $(n=47)$ after November 4, 1996 and had continuous therapy with the index drug for 9 months after initiation. The cost of index antipsychotic, psychiatric hospitalizations and outpatient care after 9 months of initiation were the outcome measures. Major limitation of this study is its limited sample size and lack of adjustment in cost comparisons for patient demographics or disease severity.

There is only one published study that includes quetiapine among head-to-head comparisons of atypical antipsychotics. This study has examined total pharmacy costs in acute care inpatient medical facilities using retrospective chart reviews. The study sample consisted of patients hospitalized between May 1, 1998 and June 30, 2000 and discharged within 30 days after admission $(\mathrm{N}=327)$. Patients initiated on risperidone ( $\mathrm{n}$ $=120)$, olanzapine $(\mathrm{n}=153)$ and quetiapine $(\mathrm{n}=54)$ within 7 days of hospital admissions were categorized in study drug groups. After adjusting for selection bias using propensity scoring methodology, the study reported that average daily pharmacy cost during the inpatient stay was $\$ 4.35$ less for risperidone and $\$ 1.41$ less for quetiapine compared to olanzapine (Mladsi et al., 2004). As the study includes patients who were discharged within 30 days of hospitalizations, the results apply only to those patients who respond to the treatment. Due to this inclusion criteria, discharge rate and length of hospital stay cannot be compared. Other outcome measures such as total inpatient costs 
were not reported. Also, there was no information or control for extent of use of other concomitant medications.

Most studies in literature have reported total costs or inpatient drug costs as outcome measures. Except for the study by Mladsi and colleagues, none of the studies have controlled for selection bias or included quetiapine in their cost comparisons. Though Mladsi and colleagues included quetiapine in their analysis, their evaluation was restricted to only pharmacy costs in an inpatient setting (Mladsi et al., 2004).

Other than curtailing costs, effectiveness of antipsychotic in improving adherence and persistence with therapy, preventing hospitalization and emergency room visits and decreasing concomitant usage of other medications is also important. Non-adherence to therapy has been shown to be major predictor of hospitalizations. Patients who are partially compliant or interrupt their antipsychotic therapy have 2-4 times greater risk of hospitalization (Weiden et al., 1995; Eaddy, Grogg, \& Locklear, 2005). Hospitalization costs represent about one-third to two-third of total healthcare costs for schizophrenia patients. Persistence with initial therapy can also play an important role in cost savings. If patients are less likely to respond upon initiation on certain antipsychotic, it is possible there is greater likelihood of future hospitalizations and emergency care in these patients. Switching to other antipsychotics will be associated with additional expenses due to nonresponse to earlier treatment. Therefore, implications of antipsychotic treatment on utilization such as adherence, persistence, emergency room visits, hospitalizations, and outpatient visits also need to be considered while evaluating antipsychotic therapy. 


\section{Antipsychotic Therapy and Economic Outcomes in Bipolar Disorder}

Addition of atypicals antipsychotics is a significant advance in bipolar disorder pharmacotherapy. However, economic evaluation studies of antipsychotic use in bipolar disorder are limited. There were only two randomized controlled trials and one retrospective database study comparing economic outcomes of atypical antipsychotic therapy in bipolar disorder.

A randomized controlled trial conducted by Namjoshi and collegues (Namjoshi et al., 2002) evaluated the cost-effectiveness of olanzapine among patients diagnosed with bipolar I disorder exhibiting manic or mixed episodes. The duration of follow-up consisted of a 3-week acute phase followed by a 49-week open label extension. During the 49-week open label extension, inpatient costs reduced by $\$ 900$ per month among patients treated with olanzapine. Another randomized control trial by Revicki and colleagues (Revicki, Paramore, Sommerville, Swann, \& Zajecka, 2003) compared divalproex and olanzapine among 120 patients with bipolar disorder Type I who were hospitalized for an acute manic episode. Patients were recruited from 21 U.S. clinical centers and randomly assigned to the divalproex or olanzapine group $(\mathrm{N}=63$ divalproex, $\mathrm{N}=57$ olanzapine). These patients were then followed in hospitals for 21 days. Patients showing clinical improvement after 21 days of therapy were further followed for 12 weeks. The study only compared 12-week outpatient cost for these patients. The divalproex group showed significantly lower outpatient costs (\$541) as compared to olanzapine patients $(\$ 1080)$.

Namjoshi and colleagues (Namjoshi et al., 2002) reported savings due to reduction in hospitalization whereas Revicki and colleagues (Revicki et al., 2003) 
reported savings in outpatient medical costs. Also, it should be noted that in the study by Revicki and colleagues, outpatient costs were compared for divalproex and olanzapine for hospitalized bipolar patients who had shown clinical improvement on their respective therapy after 21 days. Thus, it is possible that the impact of increased cost and utilization by patients not showing clinical improvement or showing deterioration due to pharmacotherapy has not been captured in this evaluation.

Gianfrancesco and colleagues used a commercial health plan's administrative data to compare mental health costs associated with risperidone, olanzapine and quetiapine treatment in bipolar disorder patients (Gianfrancesco et al., 2005). They identified treatment episodes with any of the study antipsychotics during a time period of January 1999 and April 2002. A total of 7,518 treatment episodes (29\% associated with risperidone, $30.3 \%$ associated with olanzapine and $25.7 \%$ associated with quetiapine) were identified. The unit of analysis was an antipsychotic treatment episode. Total mental healthcare costs for each treatment episode were the billed charges for mental healthcare services incurred during the treatment episode. Controlling for factors such as patient demographics, bipolar subtype, prior healthcare charges, treatment length and substance abuse, the study reports that risperidone and quetiapine incurred about $\$ 84$ and $\$ 76$ lesser mental-health related charges per patient per month compared to olanzapine. There was no significant difference in costs between risperidone and quetiapine (Gianfrancesco et al., 2005).

This study has certain limitations. The study has compared only mental-health costs incurred between the initiation of therapy and discontinuation with the therapy. Patients may discontinue treatment due to treatment failure or adverse affects of the 
therapy. Costs associated with this treatment failure would be incurred in the postdiscontinuation phase. In addition, it is not possible to identify discontinuation of a treatment if it occurs during the hospitalization. Therefore, costs associated with this hospitalization may not be included in the total costs associated with the antipsychotic used in the episode. Also, if the same patient has multiple episodes, it is possible that this patient is more severe or treatment refractory. As this person has not stabilized on earlier antipsychotic therapy, he or she may incur higher costs when initiated on another antipsychotic in the next episode. It is also likely that such patients would be initiated on newer drugs in later episodes. Therefore, treatment episodes with newer may have higher costs due to inclusion of more severe patients. The study does not control for selection bias or for the use of mood stabilizers along with the antipsychotic therapy. 


\section{CHAPTER THREE}

\section{METHODOLOGY}

An overview of antipsychotic therapy for schizophrenia and bipolar population has been provided in the previous chapters. Rationale for using Medicaid data, sample selection criteria, identification of cohorts, outcomes evaluation and sample selection bias adjustment has been explained under the conceptual framework section in chapter one. This chapter focuses on describing the data source, selection criteria, variable definitions, study design and statistical methodology.

\section{Data source}

\section{West Virginia Medicaid Claims Data}

The West Virginia Medicaid Program (WVMP) is managed by the Bureau for Medical Services of the West Virginia Department of Health and Human Resources. It is responsible for providing health care coverage to poor and disabled individuals. The West Virginia Medicaid Bureau currently contracts with ACS, Inc (based in Atlanta, GA) to serve as its claims processor. ACS maintains and operates the Medicaid Management Information Systems (MMIS), which processes provider claims and payments. MMIS data comprises of 3 files - provider files, recipient files, and claims (medical and pharmacy) files. The following is a description of these files and the specific fields (variables) contained in each file.

The provider file contains specific information regarding all health care providers who are eligible to deliver services to Medicaid recipients. This information includes a provider number, specialty and Medicaid eligibility related information. The recipient file includes information such as Medicaid number, eligibility begin date, eligibility end 
date, gender, and race. The claims file stores detailed information specific to processed claims. The medical claim file contains information, such as invoice type, provider number, recipient number, International Classification of Disease $9^{\text {th }}$ edition (ICD-9) code of diagnosis for which the service was provided, Common Procedural Terminology (CPT) code for procedures and services provided, Diagnostic Related Group (DRG) codes, date claim was submitted, date of adjudication, through-date of service, coordination of benefit code, primary carrier code, and total amount paid. The pharmacy claim file contains information such as number of days supply, metric quantity, National Drug Classification (NDC) code, generic code, therapeutic class code, refill number, provider ID number, and amount paid.

Permission to use the WV Medicaid data was obtained from the West Virginia Medicaid Bureau. The approval to conduct the study was obtained from the Institutional Review Board (IRB). The data was stored and analyzed according to the Health Insurance Portability and Accountability Act (HIPAA) standards.

\section{Data extraction and cleaning}

The West Virginia University's Rational Drug Therapy Program (RDTP) stores the West Virginia Medicaid data obtained from ACS, Inc on its server. The data needed for this study was downloaded from the server using Access and converted to SAS datasets. The extracted data was then put through cleaning and classification processes to obtain the final dataset.

The WV Medicaid organizes all its medical claims into two separate files: the outpatient claims file and the ER/ hospitalization claims file. The ER/ hospitalization claims file contains ER and hospitalization claims as well as some outpatient claims. All 
claims from the ER/ hospitalization file having DRG greater than 0 were classified as hospitalization claims. Revenue codes and hospitalization extract indicator variable indicating ER visit were used to identify ER visit. All claims occurring on the same day as ER visit were classified into ER file. ER visits leading to hospitalization were considered as hospitalization events. Therefore, ER claims that had same date as hospitalization claims were transferred to hospitalization file. Remaining claims in the $\mathrm{ER} /$ hospitalization were identified as outpatient claims and transferred to the outpatient file. The WV Medicaid's outpatient file also contained few ER claims. These claims were identified on the basis on CPT codes (99281-99285) and transferred to the ER file. In addition, duplicate claims in the medical and pharmacy files (identified by presence of subsequent negative claims) were deleted.

\section{Study population}

West Virginia Medicaid claims data for the period January $1^{\text {st }} 1998$ to December $31^{\text {st }} 2002$ was extracted for the purpose of the study. All patient-level demographic data as well as paid claims information on type of service, date of service, amount billed, length of service, and prescription drug claims were reviewed. Patients who were 65 years or older were excluded as they have Medicare as their primary payer for health services. Only patients less than or of 64 years of age are included to avoid including those patients who would become eligible for Medicare during the period of follow-up after being initially present. Managed care recipients also do not have all their utilization information in the Medicaid claims and were excluded.

The index period for the study ranged from January $1^{\text {st }} 1999-$ December $31^{\text {st }}$ 2001, to allow for 12 pre-index and 12 post-index months. From the prescription claims 
data, all paid claims information on patients receiving at least two prescriptions of antipsychotics between January $1^{\text {st }}, 1999$ and December $31^{\text {st }} 2001$ was extracted. The first date of paid claim for an antipsychotic during the index period was considered as the index prescription. The selection criteria used to derive the final study sample for the study are listed below:

\section{Selection Criteria}

- At least two prescriptions of antipsychotics between January $1^{\text {st }}, 1999$ and December $31^{\text {st }}, 2001$

- Patients who were 65 years or older were excluded as they have Medicare as their primary payer for health services

- At least 12 months of continuous enrollment with the West Virginia Medicaid before and after the index date

- Managed care recipients were excluded as they do not have all their utilization information in the Medicaid claims.

- No claims of an antipsychotic medication during a 90 days period before the index date to indicate the index prescription is an initiation of antipsychotic therapy.

- Patients initiated on clozapine were excluded. As clozapine is indicated for treatment refractory schizophrenia, patients initiated on clozapine are more likely to be more severe or inappropriately initiated on clozapine. In addition, patients initiated on ziprasidone will excluded as it was introduced towards the end of our index period in 2001 and will probably have very small sample size. 


\section{Identification of schizophrenia and bipolar disorder patients}

Schizophrenia patients will be identified by at least 1 inpatient or 2-outpatient diagnosis of schizophrenia. Schizophrenia or Schizoaffective disorder diagnosis will be defined on the basis of International Classification of Diseases $\left(9^{\text {th }}\right.$ Edition $)($ ICD-9-CM) code of 295.00 - 295.99. This algorithm to identify schizophrenia patients in Medicaid has been validated in a study by Lurie and colleagues (Lurie P et al., 1992). Based on a study by Unutzer and colleagues validating identification of bipolar disorder patients in a large HMO population, bipolar disorder patients will be identified by at least 2 or more paid claims with ICD-9-CM diagnosis for 296.00-296.19, 296.40-296.89 (Simon et al., 1999).

\section{Measurement of cost}

Total health care costs will include costs associated with both medical and mental conditions. Mental health-related costs will be identified by claims associated with diagnosis of a mental health disorder (ICD-9-CM codes 290.xx - 316.xx) or CPT codes for psychiatric services (90801-90899) and psychiatric medications. Health care costs will be categorized into inpatient, ER, outpatient, and pharmacy cost. As one-year preindex and post-index costs will be compared for patients initiated on antipsychotics anytime during the index period, all costs will be adjusted to 2002 US dollars using the medical care and prescription price index.

\section{Measurement of mental health-related variables}

Psychotherapy utilization will be identified using CPT codes (90804-90829) or CPT codes (90846-90853). Many psychiatric patients also receive medication management (identified by CPT code 90862) that includes prescribing, recommendations 
for use, and review of medication with no more than minimal medical psychotherapy. Electroconvulsive therapy will be identified using CPT codes (90870-90871). All hospitalization, ER visits, and physician office visits associated with a diagnosis of a mental health disorder (ICD-9-CM codes 290.xx - 316.xx) will be categorized as mentalhealth-related utilization. Psychiatric medications utilization will be categorized into different classes such as mood stabilizers, antidepressants, antipsychotics, anxiolytics/hypnotics/sedatives, antiparkinsons, anticholinergics, benzodiazepines and other psychiatric drugs. Drugs from each of the above class will be identified from the pharmacy claims data using the NDC codes. The provider identifier associated with the index prescription claim will be used to determine if the prescribing physician was a psychiatrist or a non-psychiatrist. Various pre-index utilization variables such as preindex ER visits, pre-index hospitalizations, pre-index physician visits, number of psychotherapy visits, number of medication management visits, and pre-index period psychiatric medication use of mood stabilizers, antipsychotics, antidepressants and benzodiazepines will be used to control for confoundings in the multivariate analysis.

\section{Patient demographics}

The Medicaid recipient data file contained information on the patient's date of birth, gender, race and county of residence. The patient's age was obtained by calculating the difference between the recipient's date of birth and the date of index prescription. As the proportion of non-Caucasian population in WV is very less, the patients were classified as Caucasians and non-Caucasians. Patient's county of residence was classified as metropolitan and non-metropolitan by using the Rural-urban Continuum Codes. The Rural-urban Continuum Codes were developed by the standard Office of 
Management and Budget (OMB) to differentiate metropolitan and non-metropolitan counties based on size, degree or urbanization and proximity to metro areas (Rural Urban Continuum codes, 2003).

\section{Co-morbidities}

It is important to control for baseline co morbid conditions that may be present among patients initiated on antipsychotics. Presence of specific mental health conditions such as major depression, mild to moderate depression, attention deficit disorder, nonschizophrenia psychosis, other affective disorders, anxiety, personality disorder, dementia, and autism in the pre-index period will be determined on the basis of ICD-9CM codes. Conditions such as arrhythmia, diabetes, hypertension, and arrhythmia that have been associated with the use of antipsychotic will also be determined from the preindex claims. Alcohol and substance abuse is also a common co-morbid condition among schizophrenia patients and can be identified on basis of ICD-9-codes (291, 292, $303,304,305.0-305.03,305.2-305.9)$ from claims data. Suicide attempts recorded in the claims data in form of ICD-9-CM code (E950-E959) will also be identified. In addition, Charlson co-morbidity index will also be used to measure co morbidity. Charlson comorbidity index is sum of the severity weights ranging from 1 to 6 that were calculated on the basis of relative risks of mortality and assigned to 19 co-morbid conditions. This index was adapted by Deyo and colleagues for use with ICD-9-CM codes from administrative claims data to predict resource utilization (Deyo, Cherkin, \& Ciol, 1992). Deyo's adaptation of Charlson co-morbidity index will be used to control for severity of other medical conditions while predicting cost and utilization. 


\section{Phase 1}

\section{Research Question 1}

To determine the annual prevalence rate of schizophrenia and bipolar disorder in West Virginia Medicaid from 1998 to 2002

The number of patients identified with the diagnosis of schizophrenia or bipolar disorder based on ICD-9 claims from the Medical claims file will be used as the numerator in the prevalence rate calculations. The denominator will be the number of people eligible under the WV Medicaid for that year. The number of people eligible under the WV Medicaid was derived from the Health Care Financing Administration (HCFA) 2082 Reports. The eligible population consists of people who have paid claims

in the Medicaid claims data as well as people who have never used/claimed any Medicaid services but were eligible for them.

\section{Research Question 2}

To determine the medical conditions among the patients initiated on antipsychotics during the study period in the West Virginia Medicaid population as well as describe patterns of distribution of certain demographic factors such as age, gender, and ethnicity in these patients

\section{Identifying Mental Health Comorbidities}

A patient will coded to be under mental health conditions listed below on the basis of ICD-9-CM diagnosis for that condition in the medical claims data during the preindex period or the index date.

- Major Depression: At least 2 or more paid claims with ICD-9-CM diagnosis for 296.20-296.39. 
- Mild to Moderate Depression: At least 2 or more paid claims with ICD-9-CM diagnosis for 300.40-300.49, 311, 309.0 and 309.1.

- Attention-Deficit Disorder: At least 2 or more paid claims with ICD-9-CM diagnosis for $314.00,314.01$ and 314.0

- Non-schizophrenia Psychosis: At least 2 or more paid claims with ICD-9-CM diagnosis for 291.00-294.99

- Other Affective Disorders: At least 2 or more paid claims with ICD-9-CM diagnosis for 296, 296.0, 296.9, 296.90 and 296.99

- Anxiety: At least 2 or more paid claims with ICD-9-CM diagnosis for 297.00297.00-297.99, 300.00-300.39, 300.5-300.99

- Personality Disorder: At least 2 or more paid claims with ICD-9-CM diagnosis for 301.00-301.99

- Dementia: At least 2 or more paid claims with ICD-9-CM diagnosis for 290.00290.99

- Autism: At least 2 or more paid claims with ICD-9-CM diagnosis for 299.0, 299.00 and 299.01

- Other Mental Health diagnosis: At least 2 or more paid claims with ICD-9-CM diagnosis for 302.00-302.99, 306.00-308.99, 309.2-310.99, 311.1-313.99, $314.2-314.99,316.00-316.99$

- Multiple Categories: 2 or more of above categories Frequencies will be calculated to estimate proportion of patients initiated on antipsychotics under each mental health diagnostic category. Patient population will be categorized on basis of age into two groups: less than 18 years and 18 years. 
Demographic characteristics of patients under each diagnostic category will be presented in terms of age (mean, median), gender (frequency $\mathrm{N}, \%$ ), and race (frequency $\mathrm{N}, \%$ ). In this exploratory analysis, the patients may suffer from multiple mental health conditions. Therefore, initiation of antipsychotic cannot be assumed to be indicated for the given mental health condition. However, proportion of patients within each mental health condition who did not have any other psychiatric co-morbidity will be reported.

\section{Research Question 3}

For schizophrenia and bipolar disorder patients, determine different types of utilization pattern of antipsychotics.

Patients would be classified into schizophrenia and bipolar disorder disease category based on ICD-9-CM diagnosis code. For schizophrenia, only patients 18 years or above will be used. For bipolar disorder, patient population will be categorized on basis of age into two groups: less than 18 years and 18 years or above. All prescription claims after initiation of antipsychotic will be reviewed to determine the pattern of use of antipsychotic.

A) Pattern of antipsychotic use among schizophrenia and bipolar patients will be described in terms of:

1) Antipsychotic Polytherapy: Polytherapy refers to a situation when a second antipsychotic is started within 15 days of the index antipsychotics and both medications were refilled at least once. Patients can be initiated on polytherapy or can switch to polytherapy in course of treatment. 
2) Antipsychotic Monotherapy: Use of only one antipsychotic at a time in the course of treatment is defined as antipsychotic monotherapy. Antipsychotic monotherapy will be classified into the following types:

a) Switching: Switching refers to a situation when the index antipsychotic is changed to another antipsychotic. Antipsychotic switching will be defined as starting a different antipsychotic after the date of the index fill while not obtaining a refill for the index antipsychotic.

Patient using the same index antipsychotic throughout the 12-month post-index period will be classified as:

b) Interrupted Therapy: Patients receiving less than $80 \%$ of total days of supply of medications during the 12-month post-index period.

c) Continuous Therapy: Patients receiving greater or equal to $80 \%$ of the total days of supply of medications during the 12-month post-index period B) Schizophrenia patients initiated on index antipsychotic therapy will be categorized into:

1. Patients who continued on index antipsychotic prescription for 3 or more months

2. Patients who changed or discontinued index antipsychotic prescription within 3 months

\section{Research Question 4}

For schizophrenia and bipolar disorder patients, determine the gaps between the refills of antipsychotics.

Gaps between the refills will be defined as number of days between the depletion date of one prescription and fill date of the subsequent prescription of the antipsychotic. 
The depletion date of a prescription will be calculated as claims fill date plus days of supply. The longest continuous gap between refills for each patient will be reported in terms of mean $( \pm \mathrm{SD})$ and median gap. Also, treatment gap categories will be created depending upon the length of the gaps. Percentage distribution of patients across the treatment gap categories will be reported.

\section{Research Question 5}

For schizophrenia and bipolar disorder patients, determine predictors of patterns of use.

Multinomial logistic regressions will be used to determine predictors of pattern of antipsychotic use among schizophrenia and bipolar patients. The dependent variable will have four mutually exclusive categories: polytherapy, switching, interrupted therapy and continuous therapy. Various predictors considered in the model would be: patient demographics (age, gender, and race), prescribing physician type, schizophrenia or bipolar subtype, other medical/mental diagnosis during the pre-index period, type of index antipsychotic, year of index antipsychotic, pre-index alcohol and substance abuse and pre-index healthcare utilization. Separate analysis will be conducted for schizophrenia and bipolar patients. Disease severity will be controlled using pre-index concomitant psychiatric medication use, mental-health related ER or physician visits, and schizophrenia or bipolar disorder-related hospitalization as covariates in regression models.

Pattern of antipsychotic use (polytherapy, switching, interrupted therapy, continuous therapy $)=$ constant $+\beta 1$ demographics $+\beta 2$ physician type $+\beta 3$ co morbidities $+\beta 4$ disease subtype $+\beta 5$ pre-index utilization $+\beta 6$ index antipsychotic type + error term 


\section{Research Question 6}

For schizophrenia and bipolar disorder patients, determine the relationship between utilization pattern of antipsychotics and total health care costs.

Heckman-2 stage technique will be used to control for observed and unobserved differences between patients having different pattern of use. The first-stage selection model will be similar to multinomial regression model in research question 5 .

First-stage selection model:

Pattern of antipsychotic use (polytherapy, switching, interrupted therapy, continuous therapy $)=$ constant $+\beta 1$ demographics $+\beta 2$ physician type $+\beta 3$ co morbidities associated with treatment $+\beta 4$ disease subtype $+\beta 5$ pre-index utilization $+\beta 6$ index antipsychotic type + error term Continuous therapy will be used as reference category for all comparisons in the multinomial logistic models. The selection factor obtained from these models will be used as a covariate in the second stage regression models.

The dependent variable in the second-stage regression will be cost. As cost is usually skewed, it will be log transformed. Control variables in the second stage regression include: age, gender, Charlson Comorbidity Index, schizophrenia/ bipolar subtype, alcohol and substance abuse, psychiatrist prescriber, pre-index mental-health care cost, type of index prescription, use of concomitant psychiatric medications, year of index prescription, pattern of antipsychotic use and selection factor.

$\underline{\text { Second-stage selection model: }}$ 
$\log ($ Cost $)=$ constant $+\beta 1$ demographics $+\beta 2$ physician type $+\beta 3$ Charlson comorbidity index $+\beta 4$ disease subtype $+\beta 5$ pre-index utilization $+\beta 6$ index antipsychotic type $+\beta 7$ pattern of antipsychotic use $+\beta 8$ selection factor + error term

Separate models will be created for schizophrenia and bipolar disorder patients.

\section{Research Question 7}

For schizophrenia and bipolar disorder patients, determine the relationship between utilization pattern of antipsychotics and mental health care utilization and costs.

Mental health-related costs will be identified by claims associated with diagnosis of a mental health disorder (ICD-9-CM codes 290.xx - 316.xx) or CPT codes for psychiatric services (90801-90899) and psychiatric medications. The first stage multinomial logistic regression model will be same as that used in research question 6 . The second stage OLS regression models will have log transformed mental health-related costs as dependent variable. Separate models will be created for schizophrenia and bipolar disorder patients.

\section{Phase 2}

\section{Cohort Selection}

Patients were classified into the study cohorts based on the index prescription during the index period (January $1^{\text {st }} 1999$-December $31^{\text {st }} 2001$ ). The first prescription of a study medication (Risperidone, Olanzapine, Quetiapine, or typical antipsychotics) during the index period was defined as the index prescription. Patients initiated on any other antipsychotics or multiple antipsychotics will be excluded from the analysis. Study Cohorts 
The study participants were divided into following 4 study cohorts based on atypical antipsychotic therapy:

- Cohort 1. Patients initiated on Risperidone

- Cohort 2. Patients initiated on Olanzapine

- Cohort 3. Patients initiated on Quetiapine

- Cohort 4. Patients initiated any typical antipsychotic (chlorpromazine, haloperidol, thioridazine, fluphenazine and others).

Intent to treat approach will be employed wherein the outcomes will be attributed to the index prescription irrespective of the medication pattern subsequent to index prescription.

\section{Reference Cohort}

Quetiapine was selected as the reference antipsychotic cohort for statistical analysis for following reasons:

- Quetiapine cohort was the most recent atypical antipsychotic cohort in our study with adequate sample size. As the index period for this study was between January $1^{\text {st }} 1999$ and December $31^{\text {st }} 2001$, there were not sufficient numbers of patients initiated on ziprasidone during this period to provide adequate sample size for statistical analyis.

- Economic evaluations comparing quetiapine to other antipsychotics were lacking in the published literature.

\section{Research Question 8}

For schizophrenia and bipolar disorder patients, to compare the impact of quetiapine with risperidone, olanzapine, and typical antipsychotics on total and mental healthcare costs 
Cost data will be collected for each of the study participants in the pre- and post index phase. Mental health-related utilization will be identified by claims associated with diagnosis of a mental health disorder (ICD-9-CM codes 290.xx - 316.xx) or CPT codes for psychiatric services (90801-90899) and psychiatric medications. Total health care costs will include all costs for medical and mental conditions.

Univariate analysis will describe resource costs of care: 1) in the pre-index period, and 2) in the post-index period. The Wilcoxon rank sum tests (equivalent to MannWhitney U test) were used to measure between-cohort differences. The Wilcoxon rank sum test is a non parametric alternative to two sample $t$ test that can be used to compare differences between cohorts for non-normal data.

Next, multivariate regression techniques, controlling for specific covariates, will be used to evaluate the association between type of atypical antipsychotic therapy used and post-index cost. Age, gender, prescribing physician specialty, index year, schizophrenia/bipolar subtype, substance and alcohol abuse, Charlson Comorbidity Score, pre-index cost and utilization, and concomitant medication use would be considered as covariates. Sample selection models will be used to adjust for sample selection bias due to treatment selection. The first stage sample selection model will be a multinomial logistic regression model predicting choice of index antipsychotic therapy.

Index antipsychotic selection (quetiapine, risperidone, olanzapine, typical antipsychotic). $=$ constant $+\beta 1$ demographics $+\beta 2$ physician type $+\beta 3$ co-morbidities $+\beta 4$ disease subtype $+\beta 5$ pre-index utilization + error term

The selection factor obtained from these models will be used as a covariate in the second stage regression models. 
$\log (\mathbf{C o s t})=$ constant $+\beta 1$ demographics $+\beta 2$ physician type $+\beta 3$ co-morbidities $/$ Charlson comorbidity index $+\beta 4$ disease subtype $+\beta 5$ pre-index utilization $+\beta 6$ index antipsychotic type $+\beta 8$ selection factor + error term

Quetiapine will be used as reference category for all comparisons in the multinomial logistic models and regression models. Separate analysis will be conducted for schizophrenia and bipolar disorder patients. For each disease group, separate models will developed for mental health-related and total healthcare cost.

\section{Research Question 9}

For schizophrenia and bipolar disorder patients, to compare the impact of quetiapine with risperidone, olanzapine, and typical antipsychotics on components of mental healthcare costs (costs associated with mental health-related inpatient, emergency room, outpatient and pharmacy services)

Univariate analysis will describe resource costs of care: 1) in the pre-index period, and 2) in the post-index period. Univariate comparisons for the differences in mental health-related inpatient, emergency room, outpatient and pharmacy costs between the index drug cohorts will be conducted using the Wilcoxon rank sum tests.

A 2-part model will be used to establish relationship between drug therapy patterns and mental health related cost categories like ED and inpatient that are likely to have zero values for some patients. The first part will consist of a logistic regression for each dependent variable (ED and inpatient) to distinguish between patients having no cost and any cost. The second part will be a multiple regression analysis conducted among the patients who have incurred greater than zero cost during the follow-up period. 


\section{Part 1:}

Any Cost $(0 /$ greater than 0$)=$ constant $+\beta 1$ demographics $+\beta 2$ physician type $+\beta 3$

Charlson comorbidity index $+\beta 4$ disease subtype $+\beta 5$ pre-index utilization $+\beta 6$ index antipsychotic type $+\beta 7$ pattern of antipsychotic use $+\beta 8$ selection factor + error term

\section{Part 2:}

$\log ($ Cost $)=$ constant $+\beta 1$ demographics $+\beta 2$ physician type $+\beta 3$ Charlson comorbidity index $+\beta 4$ disease subtype $+\beta 5$ pre-index utilization $+\beta 6$ index antipsychotic type $+\beta 7$ pattern of antipsychotic use $+\beta 8$ selection factor + error term

For dependent variables like total mental health-related costs, outpatient and pharmacy cost category that are not likely to have zero costs, multiple regression analysis will be used.

Treatment severity will be controlled using pre-index concomitant psychiatric medication use and other mental-health related healthcare utilization as covariates in regression models. Control variables in the study will include: age, gender, Charlson Comorbidity Index, schizophrenia/ bipolar subtype, alcohol and substance abuse, psychiatrist prescriber, pre-index mental- health care cost and utilization, use of concomitant psychiatric medications, and year of index prescription. As the cost data is skewed, log transformations will be used to normalize the data.

\section{Research Question 10}

For schizophrenia and bipolar patients, to compare the impact of quetiapine with risperidone, olanzapine, and typical antipsychotics on mental health-related healthcare hospitalizations 
Univariate analysis will describe number of inpatient visits and length of inpatient visits: 1) in the pre-index period, and 2) in the post-index period. The Wilcoxon rank sum tests were used to measure between-cohort differences.

Hospitalization rates calculated as the percent of patients who were hospitalized with a psychiatric diagnosis during the pre and post index period will be analyzed using Mantel-Haenszel test. Mantel-Haenszel test is used to compare proportion of people between two groups.

Time to hospitalization will be defined as the number of days from the index date to the first hospitalization. As this is time-to-event data, survival analysis procedures will be used for analysis. Kaplan Meier survival curves will be used for univariate analysis. The relative hazard of hospitalization over a follow-up period will be analyzed using Cox proportional model that will control for confounding variables.

Time to first hospitalization $=$ constant $+\beta 1$ demographics $+\beta 2$ physician type $+\beta 3$ Charlson comorbidity index $+\beta 4$ disease subtype $+\beta 5$ pre-index utilization $+\beta 6$ index antipsychotic type $+\beta 7$ pattern of antipsychotic use $+\beta 8$ selection factor + error term

Poisson regression analysis will be used for multivariate comparison of the number of inpatient visits among different cohorts.

Separate analysis will be conducted for schizophrenia and bipolar disorder patients.

\section{Research Question 11}

For schizophrenia patients and bipolar disorder patients, to compare the impact of quetiapine with risperidone, olanzapine, and typical antipsychotics on mental healthcarerelated emergency room visits 
Univariate analysis will describe ER visits: 1) in the pre-index period, and 2) in the postindex period. The Wilcoxon tests will be used to measure between-cohort differences.

ER visit rate is calculated as the percent of patients who were admitted with a psychiatric diagnosis during the pre and post index period and will be analyzed using Mantel-Haenszel test.

Time to ER visit will be defined as the number of days from the index date to the first ER visit. The relative hazard (or incidence rate) of ER visit over a follow-up period will be analyzed using Cox proportional model.

Poisson regression analysis will be conducted to compare the number of ER visits among different cohorts.

Control variables in the study will include: age, gender, prescribing physician specialty, index year, adherence to index medication, schizophrenia/bipolar subtype, Charlson Comorbidity Score, pre-index psychiatric ER visits, substance abuse, alcohol abuse, and concomitant medication use.

Separate analysis will be conducted for schizophrenia and bipolar disorder patients.

\section{Research Question 12}

For schizophrenia and bipolar disorder patients, to compare the impact of quetiapine with risperidone, olanzapine, and typical antipsychotics on mental healthcare-related outpatient visits

Univariate analysis will describe total and mental health-related physician visits, psychotherapy sessions and specific medication management psychotherapy in terms of: 
1) in the pre-index period, and 2) in the post-index period. The Wilcoxon rank sum tests will be used to measure between-cohort differences.

Poisson regression analysis will be conducted to compare the number of mental health-related physician visits among different cohorts.

Control variables in the study will include: age, gender, prescribing physician specialty, index year, schizophrenia/bipolar subtype, Charlson Comorbidity Score, preindex physician visits, substance abuse, alcohol abuse, and concomitant medication use.

Separate analysis will be conducted for schizophrenia and bipolar disorder patients.

\section{Research Question 13}

For schizophrenia and bipolar disorder patients, to compare the impact of quetiapine with risperidone, olanzapine, and typical antipsychotics on psychiatric medication utilization

Use of concomitant medications will be categorized into following classes:

Mood stabilizers, antidepressants, Anxiolytics/Hypnotics/Sedatives, antiparkinsons, anticholinergics, antipsychotics (pre-index use), clozapine and benzodiazepine.

Proportions of patients from each cohort using concomitant medications will be compared using Mantel-Haenszel test.

Separate analysis will be conducted for schizophrenia and bipolar disorder patients.

\section{Research Question 14}

For schizophrenia and bipolar disorder patients, to compare the impact of quetiapine with risperidone, olanzapine, and typical antipsychotics on patient's adherence to the index medications in the post-index period 
Adherence with the index therapy will be compared in terms of Medication possession ratio (MPR)

$\mathrm{MPR}=\underline{\text { the total days supplied for the index drug }}$

total days from index date to the date of last prescription

of index drug + days supplied on last claim

Medicaid prescription claims data does not record claims of medications

administered to patients in an inpatient setting. To adjust for this absence of record while calculating days of supply of index antipsychotic, the number of days of hospitalizations was added to the days supply of drugs for the index antipsychotic if the admit and discharge dates of the hospitalization occurred between the first fill date and end of follow up period for that index antipsychotic.

Ordinary Least Squares (OLS) regression models will be used determine the impact of choice of index antipsychotic drug on MPR. Age, gender, prescribing physician specialty, index year, schizophrenia/bipolar subtype, Charlson Comorbidity Score, schizophrenia/bipolar subtype, other mental illnesses, psychiatric hospitalization, psychiatric ER visit, substance abuse, alcohol abuse, and concomitant medication use would be considered as covariates. Separate analysis will be conducted for schizophrenia and bipolar disorder patients.

\section{Research Question 15}

For schizophrenia and bipolar disorder patients, to compare the impact of quetiapine with risperidone, olanzapine, and typical antipsychotics on subsequent index antipsychotic therapy modification 


\section{Persistence or time until treatment change}

Persistence or time until treatment change will be defined as the total days from the index prescription fill date until the occurrence of a filled prescription for any other index or a non-index antipsychotic or until discontinuation of therapy with the index drug.

\section{The relative hazard of discontinuation or switch}

The relative hazard of discontinuation or switch over a follow-up period will be analyzed using Cox proportional hazard model. Age, gender, prescribing physician specialty, index year, adherence to index medication, schizophrenia/bipolar subtype, Charleston Comorbidity Score, schizophrenia/bipolar subtype, other mental illnesses, psychiatric hospitalization, psychiatric ER visit, substance abuse, alcohol abuse, concomitant medication use, and time from date of market availability to index date would be considered as covariates. Separate analysis will be conducted for schizophrenia and bipolar disorder patients. 


\section{CHAPTER FOUR \\ RESULTS AND DISCUSSION}

The previous chapter described study objectives and methodology. This chapter presents results and discussion for each of the study objectives. The results and discussion for schizophrenia and bipolar disorder are presented together under each research question. The results are classified into phase 1 and phase 2 .

Table 1 presents the number of eligible study participants after applying each inclusion and exclusion criterion. A total of 5,297 patients were initiated on antipsychotic medications between the index period January 1st, 1999 and December $31^{\text {st }}, 2001$ in the WV Medicaid. Patients with diagnosis of schizophrenia and bipolar disorder were identified using the specified criteria. A total of 1,036 patients with schizophrenia and 832 patients with bipolar disorder were initiated on antipsychotics during the study index period. Power analysis carried out for the resulting sample size revealed greater than $80 \%$ power for the univaraite and multivariate analysis proposed in this study. 
Table 1: Application of the selection criteria and the resulting sample size

\begin{tabular}{|c|c|}
\hline Selection Criteria & $\begin{array}{c}\text { Sample Size } \\
N\end{array}$ \\
\hline $\begin{array}{l}\text { 1. At least } 2 \text { antipsychotic Rx during the index period } \\
\text { (January } 1^{\text {st }} 1999 \text {-December } 31^{\text {st }} 2001 \text { ) }\end{array}$ & 22,577 \\
\hline 2. Less than or equal to 64 years of age & 16,698 \\
\hline $\begin{array}{l}\text { 3. At least } 12 \text { months of continuous pre and post } \\
\text { enrollment }\end{array}$ & 11,021 \\
\hline 4. Excluding managed care recipients & 10,193 \\
\hline $\begin{array}{l}\text { 5. No claims of an antipsychotic during a } 90 \text { days period } \\
\text { before the index date }\end{array}$ & 5,384 \\
\hline 6. Excluding patients initiated on clozapine & 5,366 \\
\hline 7. Excluding patients initiated on ziprasidone & 5,297 \\
\hline Final Sample & 5,297 \\
\hline $\begin{array}{l}\text { 8. Identification of schizophrenia patients ( } 18 \text { years and } \\
\text { older) }\end{array}$ & 1,036 \\
\hline 9. Identification of bipolar disorder patients & 832 \\
\hline
\end{tabular}




\section{PHASE 1}

\section{Results for research objective 1}

To determine the annual prevalence rate of schizophrenia and bipolar disorder in West Virginia Medicaid from 1998 to 2002

To calculate the annual prevalence rate of schizophrenia and bipolar disorder patients, all patients with diagnosis of schizophrenia or bipolar disorder during the year (not just patients initiated on antipsychotics) were identified as numerator. The denominator was the number of people eligible under the WV Medicaid for that year. Tables $2 \mathrm{a}$ and $2 \mathrm{~b}$ present annual prevalence rates for schizophrenia and bipolar disorder in the WV Medicaid population for the years 1998-2002. Total annual prevalence of schizophrenia ranged from $0.9 \%$ to $1.5 \%$ between the year 1998 and 2002 among WV Medicaid eligible population. Total annual prevalence of bipolar disorder ranged from $0.6 \%$ to $1.7 \%$ between the year 1998 and 2002 among WV Medicaid eligible population. Annual prevalence of bipolar disorder among patients between 20 and 64 years of age increased from $1.3 \%$ in 1998 to $3.2 \%$ in 2002 . Annual prevalence of bipolar disorder among patients less than 14 years of age was $0.02 \%-0.03 \%$ and patients between 14 and 20 years of age was $0.08 \%-1.7 \%$. 
Table 2a: Annual prevalence rate for schizophrenia in the WV Medicaid (1998-2002)

\begin{tabular}{|c|c|c|c|c|c|c|c|c|c|c|}
\hline & \multicolumn{2}{|r|}{2002} & \multicolumn{2}{|r|}{2001} & \multicolumn{2}{|r|}{2000} & \multicolumn{2}{|r|}{$1999^{*}$} & \multicolumn{2}{|r|}{1998} \\
\hline & $N^{b}$ & $\begin{array}{c}P R \\
{[95 \% C I]}\end{array}$ & $N^{b}$ & $\begin{array}{c}P R \\
{[95 \% C I]}\end{array}$ & $N^{b}$ & $\begin{array}{c}P R \\
{[95 \% C I]}\end{array}$ & $N^{b}$ & $\begin{array}{c}P R \\
{[95 \% C I]}\end{array}$ & $N^{b}$ & $\begin{array}{c}P R \\
{[95 \% C I]}\end{array}$ \\
\hline \multicolumn{11}{|l|}{ Age Category } \\
\hline $\begin{array}{l}\text { Up to or equal } \\
\text { to } 14 \text { years }\end{array}$ & 27 & $\begin{array}{c}0.17 \\
{[0.10,0.23]}\end{array}$ & 23 & $\begin{array}{c}0.24 \\
{[0.14,0.34]}\end{array}$ & 34 & $\begin{array}{c}0.23 \\
{[0.15,0.31]}\end{array}$ & 77 & - & 99 & $\begin{array}{c}0.59 \\
{[0.48,0.71]}\end{array}$ \\
\hline $\begin{array}{l}\text { Greater than } 14 \\
\text { to } 20 \text { years }\end{array}$ & 110 & $\begin{array}{c}2.74 \\
{[2.23,3.25]}\end{array}$ & 112 & $\begin{array}{c}2.91 \\
{[2.37,3.45]}\end{array}$ & 142 & $\begin{array}{c}3.68 \\
{[3.07,4.28]}\end{array}$ & 114 & - & 124 & $\begin{array}{c}2.89 \\
{[2.38,3.40]}\end{array}$ \\
\hline $\begin{array}{l}\text { Greater than } 20 \\
\text { to } 64 \text { years }\end{array}$ & 4,110 & $\begin{array}{c}32.47 \\
{[31.47,33.46]}\end{array}$ & 3,995 & $\begin{array}{c}32.03 \\
{[31.03,33.02]}\end{array}$ & 4,001 & $\begin{array}{c}32.12 \\
{[31.12,33.11]}\end{array}$ & 3,154 & - & 2,854 & $\begin{array}{c}21.98 \\
{[21.18,22.79]}\end{array}$ \\
\hline
\end{tabular}


Table 2b: Annual prevalence rate for bipolar disorder in the WV Medicaid (1998-2002)

\begin{tabular}{|c|c|c|c|c|c|c|c|c|c|c|}
\hline & \multicolumn{2}{|r|}{2002} & \multicolumn{2}{|r|}{2001} & \multicolumn{2}{|r|}{2000} & \multicolumn{2}{|r|}{$1999^{*}$} & \multicolumn{2}{|r|}{1998} \\
\hline & $N b$ & $\begin{array}{c}P R \\
{[95 \% C I]}\end{array}$ & $N^{b}$ & $\begin{array}{c}P R \\
{[95 \% C I]}\end{array}$ & $N^{b}$ & $\begin{array}{c}P R \\
{[95 \% C I]}\end{array}$ & $N^{b}$ & $\begin{array}{c}P R \\
{[95 \% C I]}\end{array}$ & $N^{b}$ & $\begin{array}{c}P R \\
{[95 \% C I]}\end{array}$ \\
\hline \multicolumn{11}{|l|}{ Age Category } \\
\hline $\begin{array}{l}\text { Up to or equal } \\
\text { to } 14 \text { years }\end{array}$ & 571 & $\begin{array}{c}3.53 \\
{[3.24,3.82]}\end{array}$ & 367 & $\begin{array}{c}3.81 \\
{[3.42,4.19]}\end{array}$ & 479 & $\begin{array}{c}3.24 \\
{[2.95,3.53]}\end{array}$ & 339 & - & 260 & $\begin{array}{c}1.56 \\
{[1.37,1.75]}\end{array}$ \\
\hline $\begin{array}{l}\text { Greater than } 14 \\
\text { to } 20 \text { years }\end{array}$ & 689 & $\begin{array}{c}17.15 \\
{[15.87,18.43]}\end{array}$ & 630 & $\begin{array}{c}16.38 \\
{[15.10,17.66]}\end{array}$ & 593 & $\begin{array}{c}1,5.35 \\
{[14.11,16.58]}\end{array}$ & 451 & - & 347 & $\begin{array}{c}8.08 \\
{[7.23,8.93]}\end{array}$ \\
\hline $\begin{array}{l}\text { Greater than } 20 \\
\text { to } 64 \text { years }\end{array}$ & 4,125 & $\begin{array}{c}32.59 \\
{[31.59,33.58]}\end{array}$ & 3,525 & $\begin{array}{c}28.26 \\
{[27.33,29.19]}\end{array}$ & 3,473 & $\begin{array}{c}27.88 \\
{[26.95,28.81]}\end{array}$ & 2,243 & - & 1,723 & $\begin{array}{c}13.27 \\
{[12.64,13.90]}\end{array}$ \\
\hline $\begin{array}{l}\text { Total } \\
\text { (less than or } \\
\text { equal to } 64 \text { years } \\
\text { population) }\end{array}$ & 5,385 & $\begin{array}{c}16.39 \\
{[15.96,16.83]}\end{array}$ & 4,522 & $\begin{array}{c}17.42 \\
{[16.91,17.92]}\end{array}$ & 4,545 & $\begin{array}{c}14.61 \\
{[14.19,15.03]}\end{array}$ & 3,033 & $\begin{array}{c}8.80 \\
{[8.49,9.11]}\end{array}$ & 2,330 & $\begin{array}{c}6.87 \\
{[6.59,7.15]}\end{array}$ \\
\hline
\end{tabular}




\section{Discussion for research objective 1}

Information regarding prevalence of schizophrenia and bipolar disorder in WV Medicaid is important from a health services planning perspective. Although it has been suggested that the prevalence of these mental health disorders may be higher in low income populations, information regarding prevalence of schizophrenia and bipolar disorder in Medicaid systems is lacking in literature(Scott, 1993; Goldner, Jones, \& Waraich, 2003).

Based on a survey of 20,291 adults by National Institute of Mental Health Epidemiologic Catchment Area Program, one-year prevalence rate of schizophrenia in 1993 was reported to be $1.1 \%$ (Regier et al., 1993). Our study reports similar prevalence rates of schizophrenia $(0.9 \%-1.5 \%)$ from 1998 to 2002 in the WV Medicaid population. The rates of prevalence of both schizophrenia and bipolar disorder show steady increase from 1998 to 2002 in WV Medicaid, except for the year 2001. The higher prevalence rates in the year 2001 compared to 2002 was due to decrease in the denominator i.e. the number of people eligible under the WV Medicaid during the year 2001.

Prevalence of bipolar disorder has been estimated to range between $1 \%$ and $2 \%$ in different studies. The Epidemiologic Catchment Area (ECA) survey reported lifetime prevalence of $1.3 \%$ whereas the National Comorbidity survey has reported lifetime prevalence of 1.6\% (Bebbington \& Ramana, 1995; Kessler et al., 1994). A survey of representative sample of adult US population in 2000 revealed a very high prevalence rate of 3.7\% for bipolar disorder (Hirschfeld RMA, Calabrese JR, \& Weissman M, 2005). Our study also found that the prevalence rate of bipolar disorder had increased to $3.2 \%$ in 2002 among adults. The prevalence rates in our study may differ from other studies as it 
was calculated based on the presence of disease specific ICD-9-CM codes in the administrative data whereas other studies have reported the prevalence rates based on community surveys. The WV Medicaid patient population differs from the patient population used in other studies due to over representation of low-income and women population.

\section{Results for research objective 2}

To determine the medical conditions for which antipsychotics are being prescribed in the West Virginia Medicaid population as well as describe patterns of distribution of certain demographic factors such as age, gender, and ethnicity in patients using antipsychotics.

Tables 3a, 3b and 3c present results of an analysis to determine the mental health conditions as well as describe patterns of distribution of certain demographic factors such as age, gender, and ethnicity in patients who were initiated on antipsychotics in the West Virginia Medicaid population during the study period.

In this analysis, the patients may suffer from multiple mental health conditions. Therefore, initiation of an antipsychotic medication cannot be assumed to be indicated for the given mental health condition. However, the tables also provide information on proportion of patients within each mental health condition who did not have any other psychiatric co-morbidity. Disease conditions such as autism, non-schizophrenia psychosis, major depression, and attention-deficit disorders are of particular interest as considerable proportion of patients initiated on antipsychotics during the study period in these disease conditions do not have any other mental health co-morbidities. 
Table 3a: Exploratory analysis of mental health conditions among patients who were initiated on antipsychotics in the West Virginia Medicaid population during the study period

\begin{tabular}{|c|c|c|c|c|c|c|}
\hline & \multicolumn{2}{|c|}{$\begin{array}{c}\text { Attention-Deficut } \\
\text { Disorder } \\
N=472\end{array}$} & \multicolumn{2}{|c|}{$\begin{array}{c}\text { Major Depression } \\
\quad N=1,112\end{array}$} & \multicolumn{2}{|c|}{$\begin{array}{c}\text { Mild to Moderate } \\
\text { Depression } \\
N=665\end{array}$} \\
\hline & $\begin{array}{c}\text { Less } \\
\text { than } 18 \\
\text { years } \\
n=104\end{array}$ & $\begin{array}{c}18 \text { years } \\
\text { and } \\
\text { above } \\
n=368\end{array}$ & $\begin{array}{c}\text { Less } \\
\text { than } \\
18 \text { years } \\
n=212 \\
\end{array}$ & $\begin{array}{l}18 \text { years } \\
\text { and } \\
\text { above } \\
n=900\end{array}$ & $\begin{array}{c}\text { Less } \\
\text { than } 18 \\
\text { years } \\
n=145\end{array}$ & $\begin{array}{l}18 \text { years } \\
\text { and } \\
\text { above } \\
n=520\end{array}$ \\
\hline $\begin{array}{l}\text { No other } \\
\text { p sychiatric co- } \\
\text { morbidities }\end{array}$ & $\begin{array}{c}25 \\
(22.0 \%)\end{array}$ & $\begin{array}{c}95 \\
(25.8 \%)\end{array}$ & $\begin{array}{c}56 \\
(26.4 \%)\end{array}$ & $\begin{array}{c}242 \\
(26.9 \%)\end{array}$ & $\begin{array}{c}21 \\
(14.5 \%)\end{array}$ & $\begin{array}{c}59 \\
(11.3 \%)\end{array}$ \\
\hline $\begin{array}{l}\text { Age in years } \\
\text { (mean/std, median) }\end{array}$ & $\begin{array}{c}14.1 / 2.7 \\
15.0\end{array}$ & $\begin{array}{l}33.5 / 10 \\
4,32.5\end{array}$ & $\begin{array}{c}11.6 \\
3.4,12.0\end{array}$ & $\begin{array}{c}42.2 / 11 \\
6,42.0\end{array}$ & $\begin{array}{l}12.1 / 3.4, \\
13.0\end{array}$ & $\begin{array}{l}41.2 / 11.1, \\
41.0\end{array}$ \\
\hline \multicolumn{7}{|l|}{ Gender } \\
\hline Males & $\begin{array}{c}65 \\
(62.5 \%)\end{array}$ & $\begin{array}{c}159 \\
(43.2 \%)\end{array}$ & $\begin{array}{c}141 \\
(66.5 \%)\end{array}$ & $\begin{array}{c}361 \\
(40.1 \%)\end{array}$ & $\begin{array}{c}87 \\
(60.0 \%)\end{array}$ & $\begin{array}{c}208 \\
(40.0 \%)\end{array}$ \\
\hline Females & $\begin{array}{c}37 \\
(35.6 \%)\end{array}$ & $\begin{array}{c}207 \\
(56.3 \%)\end{array}$ & $\begin{array}{c}69 \\
(32.6 \%)\end{array}$ & $\begin{array}{c}532 \\
(59.1 \%)\end{array}$ & $\begin{array}{c}53 \\
(36.5 \%)\end{array}$ & $\begin{array}{c}309 \\
(59.4 \%)\end{array}$ \\
\hline Missing & $\begin{array}{c}2 \\
(1.9 \%)\end{array}$ & $\begin{array}{c}2 \\
(0.5 \%)\end{array}$ & $\begin{array}{c}2 \\
(0.9 \%)\end{array}$ & $\begin{array}{c}7 \\
(0.8 \%)\end{array}$ & $\begin{array}{c}5 \\
(3.5 \%)\end{array}$ & $\begin{array}{c}3 \\
(0.6 \%)\end{array}$ \\
\hline \multicolumn{7}{|l|}{ Race } \\
\hline Caucasians & $\begin{array}{c}94 \\
(90.5 \%)\end{array}$ & $\begin{array}{c}340 \\
(92.4 \%)\end{array}$ & $\begin{array}{c}196 \\
(92.5 \%)\end{array}$ & $\begin{array}{c}842 \\
(93.6 \%)\end{array}$ & $\begin{array}{c}133 \\
(91.7 \%)\end{array}$ & $\begin{array}{c}480 \\
(7.7 \%)\end{array}$ \\
\hline Others & $\begin{array}{c}10 \\
(9.5 \%)\end{array}$ & $\begin{array}{c}28 \\
(7.6 \%)\end{array}$ & $\begin{array}{c}16 \\
(7.5 \%)\end{array}$ & $\begin{array}{c}58 \\
(6.4 \%)\end{array}$ & $\begin{array}{c}12 \\
(8.3 \%)\end{array}$ & $\begin{array}{c}40 \\
(92.3 \%)\end{array}$ \\
\hline
\end{tabular}

Index Drug Category

Typical

Antipsychotics

$\begin{array}{cccccc}11^{*} & 95^{*} & 18^{*} & 278^{*} & 13^{*} & 158^{*} \\ (10.6 \%) & (25.8 \%) & (8.5 \%) & (30.9 \%) & (9.0 \%) & (30.4 \%)\end{array}$


Table 3a: Exploratory analysis of mental health conditions among patients who were initiated on antipsychotics in the West Virginia Medicaid population during the study period (contd.)

\begin{tabular}{|c|c|c|c|c|c|c|}
\hline & \multicolumn{2}{|c|}{$\begin{array}{c}\text { Attention-Deficit } \\
\text { Disorder } \\
N=472\end{array}$} & \multicolumn{2}{|c|}{$\begin{array}{c}\text { Major Depression } \\
\quad N=1,112\end{array}$} & \multicolumn{2}{|c|}{$\begin{array}{c}\text { Mild to Moderate } \\
\text { Depression } \\
N=665\end{array}$} \\
\hline & $\begin{array}{c}\text { Less } \\
\text { than } 18 \\
\text { years } \\
n=104 \\
\end{array}$ & $\begin{array}{c}18 \text { years } \\
\text { and } \\
\text { above } \\
n=368\end{array}$ & $\begin{array}{c}\text { Less } \\
\text { than } \\
18 \text { years } \\
n=212 \\
\end{array}$ & $\begin{array}{c}18 \text { years } \\
\text { and } \\
\text { above } \\
n=900\end{array}$ & $\begin{array}{c}\text { Less } \\
\text { than } 18 \\
\text { years } \\
n=145 \\
\end{array}$ & $\begin{array}{c}18 \text { years } \\
\text { and } \\
\text { above } \\
n=520\end{array}$ \\
\hline Risperidone & $\begin{array}{c}53^{*} \\
(51.0 \%)\end{array}$ & $\begin{array}{c}82^{*} \\
(22.3 \%)\end{array}$ & $\begin{array}{l}139^{*} \\
(65.6 \%)\end{array}$ & $\begin{array}{l}180^{*} \\
(20.0 \%)\end{array}$ & $\begin{array}{c}77^{*} \\
(53.1 \%)\end{array}$ & $\begin{array}{l}112^{*} \\
(21.5 \%)\end{array}$ \\
\hline Olanzapine & $\begin{array}{c}30^{*} \\
(28.9 \%)\end{array}$ & $\begin{array}{l}132^{*} \\
(35.9 \%)\end{array}$ & $\begin{array}{c}44^{*} \\
(20.8 \%)\end{array}$ & $\begin{array}{l}300 \% \\
(33.3 \%)\end{array}$ & $\begin{array}{c}41^{*} \\
(28.3 \%)\end{array}$ & $\begin{array}{l}177^{*} \\
(34.0 \%)\end{array}$ \\
\hline Quetiapine & $\begin{array}{c}9 * \\
(8.7 \%)\end{array}$ & $\begin{array}{c}54^{*} \\
(14.7 \%)\end{array}$ & $\begin{array}{c}9 \% \\
(4.3 \%)\end{array}$ & $\begin{array}{l}132^{*} \\
(14.7 \%)\end{array}$ & $\begin{array}{l}13^{*} \\
(9.0 \%)\end{array}$ & $\begin{array}{c}68 \% \\
(13.1 \%)\end{array}$ \\
\hline Combination & $\begin{array}{c}1 \\
(1.0 \%)\end{array}$ & $\begin{array}{c}5 \\
(1.4 \%)\end{array}$ & $\begin{array}{c}2 \\
(0.9 \%)\end{array}$ & $\begin{array}{c}10 \\
(1.1 \%)\end{array}$ & $\begin{array}{c}1 \\
(.7 \%)\end{array}$ & $\begin{array}{c}5 \\
(1.0 \%)\end{array}$ \\
\hline
\end{tabular}

${ }^{*}$ Results of chi-square analysis indicate significant differences between the index drug categ ory within the respective age groups at $p<0.05$ 
Table 3b: Exploratory analysis of mental health conditions among patients who were initiated on antipsychotics in the West Virginia Medicaid population during the study period

\begin{tabular}{|c|c|c|c|c|c|c|}
\hline & \multicolumn{2}{|c|}{$\begin{array}{l}\text { Anxiety } \\
N=837\end{array}$} & \multicolumn{2}{|c|}{$\begin{array}{c}\text { Personality Disorder } \\
N=284\end{array}$} & \multicolumn{2}{|c|}{$\begin{array}{l}\text { Autism } \\
N=51\end{array}$} \\
\hline & $\begin{array}{c}\text { Less than } \\
1 S_{\text {years }} \\
n=170\end{array}$ & $\begin{array}{c}18 \text { years } \\
\text { and above } \\
n=667\end{array}$ & $\begin{array}{c}\text { Less than } \\
1 S_{\text {years }} \\
n=55\end{array}$ & $\begin{array}{c}18 \text { years } \\
\text { and above } \\
n=299\end{array}$ & $\begin{array}{c}\text { Less than } \\
1 s_{\text {years }} \\
n=7\end{array}$ & $\begin{array}{c}18 \text { years } \\
\text { and a above } \\
n=44\end{array}$ \\
\hline $\begin{array}{l}\text { No other } \\
\text { psychiatric } \\
\text { co- } \\
\text { morbidities }\end{array}$ & $\begin{array}{c}33 \\
(19.4 \%)\end{array}$ & $\begin{array}{c}115 \\
(17.2 \%)\end{array}$ & $\begin{array}{c}1 \\
(1.8 \%)\end{array}$ & $\begin{array}{c}10 \\
(3.3 \%)\end{array}$ & $\begin{array}{c}5 \\
(71.4 \%)\end{array}$ & $\begin{array}{c}26 \\
(59.1 \%)\end{array}$ \\
\hline $\begin{array}{l}\text { Age in years } \\
\text { (mean/std, } \\
\text { median) }\end{array}$ & $\begin{array}{c}11.4 / 3.4= \\
11.0\end{array}$ & $\begin{array}{c}42.3 / 10.9 \\
42.0\end{array}$ & $\begin{array}{c}11.9 / 3.4, \\
13.0\end{array}$ & $\begin{array}{c}41.5 / 11.8 \\
41.0\end{array}$ & $\begin{array}{c}13.4 / 1.7 \\
13.0\end{array}$ & $\begin{array}{c}37.4 / 11.4, \\
36.0\end{array}$ \\
\hline \multicolumn{7}{|l|}{ Gender } \\
\hline Males & $\begin{array}{c}119 \\
(70.0 \%)\end{array}$ & $\begin{array}{c}259 \\
(38.8 \%)\end{array}$ & $\begin{array}{c}31 \\
(56.3 \%)\end{array}$ & $\begin{array}{c}110 \\
(36.7 \%)\end{array}$ & $\begin{array}{c}6 \\
(85.7 \%)\end{array}$ & $\begin{array}{c}17 \\
(38.6 \%)\end{array}$ \\
\hline Females & $\begin{array}{c}49 \\
(28.8 \%)\end{array}$ & $\begin{array}{c}400 \\
(59.9 \%)\end{array}$ & $\begin{array}{c}22 \\
(40.0 \%)\end{array}$ & $\begin{array}{c}188 \\
(62.8 \%)\end{array}$ & $\begin{array}{c}1 \\
(14.3 \%)\end{array}$ & $\begin{array}{c}27 \\
(61.4 \%)\end{array}$ \\
\hline Missing & $\begin{array}{c}2 \\
(1.1 \%)\end{array}$ & $\begin{array}{c}8 \\
(1.2 \%)\end{array}$ & $\begin{array}{c}2 \\
(3.6)\end{array}$ & $\begin{array}{c}1 \\
(0.3 \%)\end{array}$ & - & - \\
\hline \multicolumn{7}{|l|}{ Race } \\
\hline Caucasians & $\begin{array}{c}155 \\
(91.2 \%)\end{array}$ & $\begin{array}{c}618 \\
(92.6 \%)\end{array}$ & $\begin{array}{c}53 \\
(96.4 \%)\end{array}$ & $\begin{array}{c}281 \\
(93.9 \%)\end{array}$ & $\begin{array}{c}6 \\
(85.7 \%)\end{array}$ & $\begin{array}{c}40 \\
(90.9 \%)\end{array}$ \\
\hline Others & $\begin{array}{c}15 \\
(8.8 \%)\end{array}$ & $\begin{array}{c}49 \\
(7.4 \%)\end{array}$ & $\begin{array}{c}2 \\
(3.6 \%)\end{array}$ & $\begin{array}{c}18 \\
(6.1 \%)\end{array}$ & $\begin{array}{c}1 \\
(14.3 \%)\end{array}$ & $\begin{array}{c}4 \\
(9.1 \%)\end{array}$ \\
\hline \multicolumn{7}{|c|}{ Index drug Category } \\
\hline $\begin{array}{l}\text { Typical } \\
\text { Antipsychotic }\end{array}$ & $\begin{array}{c}12 \\
(7.0 \%)\end{array}$ & $\begin{array}{c}225^{*} \\
(33.7 \%)\end{array}$ & $\begin{array}{c}2 \\
(3.6 \%)\end{array}$ & $\begin{array}{c}88^{*} \\
(29.4 \%)\end{array}$ & $\begin{array}{c}1 \\
(14.2 \%)\end{array}$ & $\begin{array}{c}13 \\
(29.5 \%)\end{array}$ \\
\hline
\end{tabular}


Table 3b: Exploratory analysis of mental health conditions among patients who were initiated on antipsychotics in the West Virginia Medicaid population during the study period (contd.)

\begin{tabular}{|c|c|c|c|c|c|c|}
\hline & \multicolumn{2}{|c|}{$\begin{array}{l}\text { A nuiety } \\
N=837\end{array}$} & \multicolumn{2}{|c|}{$\begin{array}{c}\text { Personality Disar der } \\
N=284\end{array}$} & \multicolumn{2}{|c|}{$\begin{array}{l}\text { Autism } \\
N=51\end{array}$} \\
\hline & $\begin{array}{c}\text { Less than } \\
1 S_{\text {years }} \\
n=170\end{array}$ & $\begin{array}{c}18 \text { years } \\
\text { and above } \\
n=667\end{array}$ & $\begin{array}{c}\text { Less than } \\
18 \text { years } \\
n=55\end{array}$ & $\begin{array}{c}18 \text { years } \\
\text { and above } \\
n=299\end{array}$ & $\begin{array}{c}\text { Less than } \\
1 S_{\text {years }} \\
n=7\end{array}$ & $\begin{array}{c}18 \text { years } \\
\text { and above } \\
n=44\end{array}$ \\
\hline Risperidone & $\begin{array}{c}107^{*} \\
(62.9 \%)\end{array}$ & $\begin{array}{c}121^{*} \\
(18.3 \%)\end{array}$ & $\begin{array}{c}32 \\
(58.1 \%)\end{array}$ & $\begin{array}{c}62^{*} \\
(20.7 \%)\end{array}$ & $\begin{array}{c}3 \\
(42.8 \%)\end{array}$ & $\begin{array}{c}8 \\
(18.1 \%)\end{array}$ \\
\hline Olanzapine & $\begin{array}{c}38^{*} \\
(22.3 \%)\end{array}$ & $\begin{array}{c}225^{*} \\
(33.7 \%)\end{array}$ & $\begin{array}{c}13 \\
(23.6 \%)\end{array}$ & $\begin{array}{c}109^{*} \\
(36.4 \%)\end{array}$ & $\begin{array}{c}2 \\
(28.5 \%)\end{array}$ & $\begin{array}{c}16 \\
(36.3 \%)\end{array}$ \\
\hline Quetiapine & $\begin{array}{c}12 \\
(7.0 \%)\end{array}$ & $\begin{array}{c}89^{*} \\
(13.3 \%)\end{array}$ & $\begin{array}{c}5 \\
(9.0 \%)\end{array}$ & $\begin{array}{c}37^{*} \\
(12.3 \%)\end{array}$ & $\begin{array}{c}1 \\
(14.2 \%)\end{array}$ & $\begin{array}{c}7 \\
(15.9 \%)\end{array}$ \\
\hline Combination & $\begin{array}{c}1 \\
(0.8 \%)\end{array}$ & $\begin{array}{c}7 \\
(1.0 \%)\end{array}$ & $\begin{array}{c}3 \\
(5.4 \%)\end{array}$ & $\begin{array}{c}3 \\
(1.0 \%)\end{array}$ & - & - \\
\hline
\end{tabular}

${ }^{*}$ Results of chi-square analysis indica te significant differences between the index drug category within the respective age groups at $\mathrm{p}<0.05$ 
Table 3c: Exploratory analysis of mental health conditions among patients who were initiated on antipsychotics in the West Virginia Medicaid population during the study period

\begin{tabular}{|c|c|c|c|c|}
\hline & \multicolumn{2}{|c|}{$\begin{array}{l}\text { Dementia } \\
\quad N=27\end{array}$} & \multicolumn{2}{|c|}{$\begin{array}{c}\text { Non-schizophrenia } \\
\text { Pychosis } \\
N=220 \\
\end{array}$} \\
\hline & $\begin{array}{c}\text { Less than } \\
18 \text { years } \\
n=10\end{array}$ & $\begin{array}{c}\text { Less } \\
\text { than } 18 \\
\text { years } \\
n=56\end{array}$ & $\begin{array}{c}\text { Less than } \\
18 \text { years } \\
n=56\end{array}$ & $\begin{array}{c}18 \text { years } \\
\text { and above } \\
n=692\end{array}$ \\
\hline $\begin{array}{l}\text { No other psychiatric co- } \\
\text { morbidities }\end{array}$ & $\begin{array}{c}1 \\
(10.0 \%)\end{array}$ & $\begin{array}{c}18 \\
(32.1 \%)\end{array}$ & $\begin{array}{c}18 \\
(32.1 \%)\end{array}$ & - \\
\hline $\begin{array}{l}\text { Age in years (mean/std, } \\
\text { median) }\end{array}$ & $\begin{array}{c}13.5 / 3.5 \\
14.5\end{array}$ & $\begin{array}{c}12.2 / 3.1 \\
12.0\end{array}$ & $\begin{array}{c}12.2 / 3.1 \\
12.0\end{array}$ & $\begin{array}{c}37.3 / 11.2 \\
37.0\end{array}$ \\
\hline \multicolumn{5}{|l|}{ Gender } \\
\hline Males & $\begin{array}{c}7 \\
(70.0 \%)\end{array}$ & $\begin{array}{c}34 \\
(60.7 \%)\end{array}$ & $\begin{array}{c}34 \\
(60.7 \%)\end{array}$ & $\begin{array}{c}280 \\
(40.5 \%)\end{array}$ \\
\hline Females & $(30.0 \%)$ & $\begin{array}{c}21 \\
(37.5 \%)\end{array}$ & $\begin{array}{c}21 \\
(37.5 \%)\end{array}$ & $\begin{array}{c}405 \\
(58.5 \%)\end{array}$ \\
\hline Missing & - & $\begin{array}{c}1 \\
(1.8 \%)\end{array}$ & $\begin{array}{c}1 \\
(1.8 \%)\end{array}$ & $\begin{array}{c}7 \\
(1.0 \%)\end{array}$ \\
\hline \multicolumn{5}{|l|}{ Race } \\
\hline Caucasians & $\begin{array}{c}10 \\
(100.0 \%)\end{array}$ & $\begin{array}{c}50 \\
(89.2 \%)\end{array}$ & $\begin{array}{c}50 \\
(89.2 \%)\end{array}$ & $\begin{array}{c}635 \\
(91.8 \%)\end{array}$ \\
\hline Others & - & $\begin{array}{c}6 \\
(10.8 \%)\end{array}$ & $\begin{array}{c}6 \\
(10.8 \%)\end{array}$ & $\begin{array}{c}57 \\
(8.2 \%)\end{array}$ \\
\hline \multicolumn{5}{|l|}{ Index Drug Category } \\
\hline Typical Antipsychotics & - & $\begin{array}{c}4 \\
(7.2 \%)\end{array}$ & $\begin{array}{c}4 \\
(7.2 \%)\end{array}$ & $\begin{array}{l}182^{*} \\
(26.3 \%)\end{array}$ \\
\hline
\end{tabular}


Table 3c: Exploratory analysis of mental health conditions among patients who were initiated on antipsychotics in the West Virginia Medicaid population during the study period (contd.)

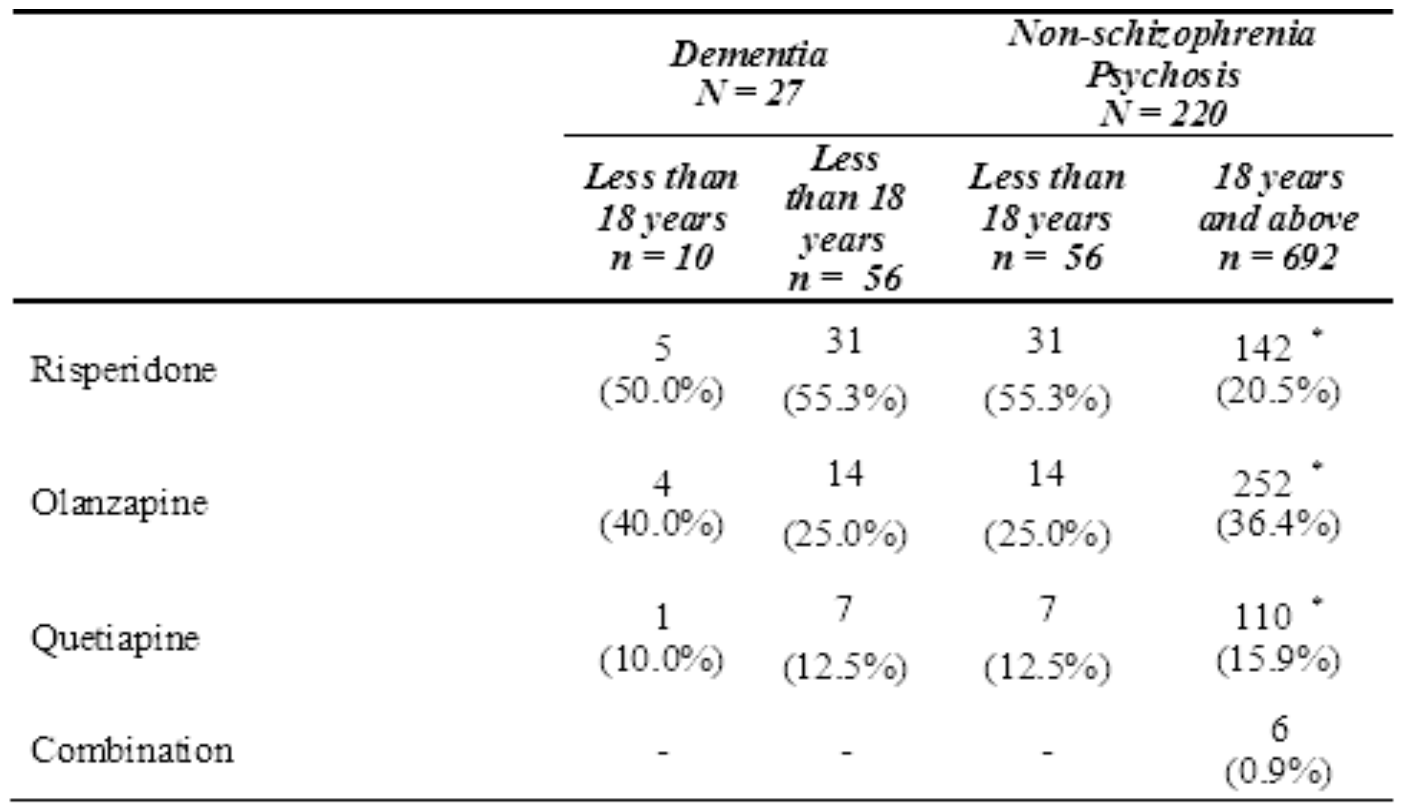

${ }^{*}$ Results of chi-square analysis indicate significant differences between the index dug category wi thin the respective age groups at $p<0.05$ 
For example, a total of 1,112 patients who were initiated on antipsychotics between 1999 and 2001 had major depression. Of these, about $26 \%$ did not have diagnosis of any other mental health disorder (except major depression) in the 12-month pre-index period suggesting that initiation of antipsychotic may have been for major depression. Similarly, more than $50 \%$ of patients with autism who were initiated on antipsychotics did not have any other co-morbidities.

For most mental health conditions (non-schizophrenic psychosis, attention deficit disorder, major depression, mild to moderate, depression, anxiety, other mental health disorders), higher proportion of patients who are less than 18 years of age were initiated on risperidone compared to any other antipsychotic.

For most mental health conditions (non-schizophrenic psychosis, attention deficit disorder, major depression, mild to moderate, depression, anxiety, personality disorder, other affective disorders, autism, other mental health disorders), higher proportion of patients who are 18 years or older were initiated on olanzapine compared to any other antipsychotic. Results of Chi-square analysis are not presented for cohorts with inadequate sample size.

\section{Discussion for research objective 2}

The results of our study indicate that a significant proportion of patients were probably initiated on antipsychotic therapy for off-label indications such as autism, nonschizophrenia psychosis, major depression and attention-deficit disorders. A study by Cooper and colleagues reported that greater numbers of adolescents are being initiated on atypical antipsychotics for conditions such as attention-deficit/hyperactivity disorder, conduct disorder, and affective disorders (Cooper et al., 2004). Other studies have also 
reported incidences of off-label antipsychotic use for depression, attentiondeficit/hyperactivity disorder, affective disorders, and autism (Barbee et al., 2004; Cooper et al., 2004; Liperoti et al., 2003). These results suggest that the prevalence of antipsychotic use in other conditions is present in clinical practice even though clinical evidence regarding risks and benefits of this practice is lacking. It is likely that antipsychotics are being used concomitantly along with other psychotropic medications indicated with these conditions.

It is important to study whether the use of antipsychotics adds any therapeutic benefit to the traditional treatment of conditions that are not yet indicated for antipsychotic use. Physicians involved in treating patients using antipsychotics can provide more information regarding outcomes of this practice. Pharmacists should be made aware of increasing use of antipsychotics for other indications and encouraged to monitor these patients regularly.

\section{Results for research objective 3}

For schizophrenia and bipolar disorder patients, determine different types of utilization pattern of antipsychotics.

$\underline{\text { Schizophrenia }}$

Table 4a describes the patterns of antipsychotic use in the 12-month follow-up period among schizophrenia patients who are greater than 18 years of age. The patterns of antipsychotic use were classified into mutually exclusive categories: 1) initial polytherapy, 2) later polytherapy, 3) switch, 4) interrupted, and 5) continuous therapy. Results of chi-square analysis revealed that there are significantly different patterns of antipsychotic use prevalent in the schizophrenia population. 
About $3.6 \%$ of patients were initiated on polytherapy (i.e received a combination of two or more antipsychotics). About $8.4 \%$ of patients switched to polytherapy during the course of their treatment. Therefore, our study population showed $12.0 \%$ of patients on polytherapy during the 12-month period after initiation of antipsychotic treatment. The remaining $88.0 \%$ of patients received antipsychotic monotherapy during the 12 month period after initiation of antipsychotic treatment.

About $12.7 \%$ of patients switched from the index antipsychotic to another antipsychotic. A large proportion of our study population (56.8\% ) received less than $80 \%$ days supply of index antipsychotic in the 12 -month post index period or interrupted therapy. About $18.5 \%$ of patients received continuous therapy, i.e. they received at least $80 \%$ days supply of index antipsychotic in the 12-month post index period.

Table $4 \mathrm{~b}$ provides the proportion of patients who continued on index antipsychotic of three or more months (trial duration based on recommendations by the Texas Medication Algorithm Project (TMAP) guidelines). About $77.3 \%$ of patients continued on index antipsychotic prescription for 3 or more months. About $22.7 \%$ of patients changed or discontinued index antipsychotic prescription within 3 months. The average duration of index antipsychotic use was $242.1( \pm 136.9)$ days. 
Table 4a: Pattern of antipsychotic use in the 12-month follow-up period among Schizophrenia patients (18 years and above)

\begin{tabular}{lc}
\multicolumn{1}{c}{ Pattern of antipsychotic use } & $\begin{array}{c}\text { Number of Patients (\%) } \\
\text { N=1,036 }\end{array}$ \\
\hline Antipsychotic Polytherapy & $37(3.6 \%)$ \\
Initial Polytherapy & $87(8.4 \%)$ \\
Later Polytherapy & \\
Antipsychotic Monotherapy & $132(12.7 \%)$ \\
Switch & $588(56.8 \%)$ \\
Interrupted & $192(18.5 \%)$ \\
Continuous & \\
\hline
\end{tabular}


Table 4b: Duration of index antipsychotic prescription use among schizophrenia patients

$$
N=999 *
$$

Duration of index antipsychotic use in days

Mean $( \pm$ std $)$

Median

$242.1( \pm 136.9)$

293.0

Patients who continued on index antipsychotic prescription for 3 or more months

n $(\%)$

$772(77.3 \%)$

Patients who changed or discontinued index antipsychotic prescription within 3 months n $(\%)$

* thirty seven patients initiated on polytherapy were deleted from this analysis. 


\section{Bipolar Disorder}

Table 5 describes the patterns of antipsychotic use in the 12-month follow-up period among bipolar patients. Results of chi-square analysis revealed that there are significantly different patterns of antipsychotic use prevalent among bipolar disorder patients who are18 years and older and less than 18 years. Initiation on antipsychotic polytherapy was seen in only $0.8 \%$ of bipolar patients. About $7.2 \%$ of 18 years and older patients and about $10.1 \%$ of less than 18 years old patients switched to polytherapy during course of their treatment.

About $13.3 \%$ of patients switched from index antipsychotic use to another antipsychotic. About $56.3 \%$ of 18 years and older patients and about $66.9 \%$ of less than 18 years old patients had received interrupted therapy. About $21.6 \%$ of 18 years and older patients and about $12.2 \%$ of less than 18 years old patients received antipsychotic adherent therapy during the post-index period. 
Table 5: Pattern of antipsychotic use in the 12-month follow-up period among bipolar disorder patients

$\begin{array}{ccc}18 \text { years and above } \\ n=684 & \text { Less than } 18 \text { years } & \text { Total } \\ n=148 & N=832\end{array}$

\begin{tabular}{lccc}
\hline \multicolumn{1}{l}{ Antipsychotic Polytherapy } & & & \\
Initial Polytherapy & $6(0.9 \%)$ & $1(0.7 \%)$ & $7(0.8 \%)$ \\
Later Polytherapy & $49(7.2 \%)$ & $15(10.1 \%)$ & $64(7.7 \%)$ \\
Antipsychotic Monotherapy & & \\
Switch & $96(14.0 \%)$ & $15(10.1 \%)$ & $111(13.3 \%)$ \\
Interrupted & $99(66.9 \%)$ & $484(58.2 \%)$ \\
Continuous & $385(56.3 \%)$ & $18(12.2 \%)$ & $166(20.0 \%)$ \\
\hline
\end{tabular}




\section{Discussion for research objective 3}

$\underline{\text { Schizophrenia }}$

Our study reports about $12.0 \%$ overall polytherapy among schizophrenia patients initiated on antipsychotics between 1999 and 2001 in the WV Medicaid. Varying rates of polytherapy have been found in published literature depending upon study population, data used, the year of study, and definitions of polytherapy. Most studies based on outpatient prescription use report lower rates of polytherapy compared to studies based on inpatient prescription use indicating that the practice of antipsychotic polytherapy is more prevalent during inpatient treatment. A study by Loosbrock and colleagues conducted using am employer claims data from 1997 reported similar polytherapy rates as our study (Loosbrock et al., 2003). They found that about $2 \%$ of patients had been initiated on polytherapy and 7\% patients had switched to polytherapy during one-year follow-up period. About $6.8 \%$ antipsychotic polytherapy was reported among schizophrenia patients from Veteran Administration in 1999(Leslie et al., 2001). Prevalence of antipsychotic polytherapy among Rhode Island Medicaid's elderly and disabled population was about 10\% in 2003 (Kogut et al., 2005). Clark and colleagues report increasing rates of polytherapy from $5.7 \%$ in 1995 to $24.5 \%$ in 1999 among schizophrenia patients in the New Hampshire Medicaid(Clark et al., 2002). Ganguly and colleagues have reported about 40\% antipsychotic polytherapy between 1998 and 2000 in the Georgia Medicaid and California Medicaid Schizophrenia patients (Ganguly et al., 2004). This is the highest prevalence of polytherapy reported based on an outpatient prescription record. It may be due to identification of polytherapy based on an episode of polytherapy anytime between 1998 and 2000. Studies evaluating antipsychotics using 
inpatient prescription records have found rates of polytherapy between $16 \%$ to $45 \%$ (Schumacher et al., 2003; Procyshyn et al., 2001; Procyshyn et al., 2004; McCue et al., 2003).

The rate of switching in the 12-month post-index period after initiation on antipsychotic was $12.7 \%$ in our study. Loosbrock and colleagues found about $13 \%$ rates of switching in 1997 among schizophrenia patients from an employer based claims data (Loosbrock et al., 2003). Williams and colleagues have reported a 25\% rate of switching observed in an indigent Indianapolis community patients who were initiated on antipsychotics in 1995 (Williams et al., 1999). As majority of their patient population $(88 \%)$ were on typical antipsychotics, it is possible that rates of switching increased as the use of atypical became more widespread. McComb and colleagues have reported a $47 \%$ rate of switching to another antipsychotic or augmenting with another antipsychotic in the California Medicaid population between 1987 and 1996 (McCombs et al., 1999b). The higher rate of switching in their study can be attributed to the episode of care methodology and possible differential rates of switching occurring between 1987 and 1996. In addition, about $98 \%$ of their study population was on typical antipsychotics. Only $18.5 \%$ of schizophrenia patients in our study received continuous index antipsychotic therapy. The rate of continuous therapy in our study is comparable to $11.6 \%$ of patients in the McComb study of California Medicaid patients (McCombs et al., 1999b). Medication adherence is usually low in the schizophrenia population due to reasons such as impaired awareness of disease, alcohol and substance abuse, and medication side-effects (Lacro, Dunn, Dolder, Leckband, \& Jeste, 2002; Hudson et al., 2004; Van Putten, 1974). The $71 \%$ monotherapy found in our study is similar to $71.5 \%$ 
reported by William and colleagues and higher than the 52\% reported by Loosbrock and colleagues (Williams et al., 1999; Loosbrock et al., 2003). Loosbrock and colleagues also reported in their study that $67.1 \%$ of patients received interrupted therapy with gaps of 1 to 11 months. Our study defines interrupted therapy in terms of patients who have received less than $80 \%$ days supply of index antipsychotic in the 12 -month post index period. This definition accounts for non-adherence to therapy due to large continuous gaps in the therapy or some intermittent missed days of therapy. We found $56.8 \%$ patients having interrupted therapy according to our definition. In general, the differences in the patterns of use across the study can be due to the differences in study methodology and definition of the pattern, year of study and proportion of patients on typical antipsychotics.

\section{$\underline{\text { Bipolar Disorder }}$}

Our study reports $7.7 \%$ prevalence of antipsychotic polypharmacy among patients initiated on antipsychotics during the index period 1999-2001. A retrospective review of psychiatric medications among patients discharged from a tertiary care psychiatric facility had revealed $29.9 \%$ rate of antipsychotic polytherapy among bipolar patients (Procyshyn et al., 2004). Compared to our study, antipsychotic polypharmacy rates may have been higher among patients discharged from hospital due to disease severity. Higher rates antipsychotic polypharmacy were also reported by studies based on inpatient prescription use among schizophrenia patients(Schumacher et al., 2003; Procyshyn et al., 2001; Procyshyn et al., 2004; McCue et al., 2003).

Rates of interrupted therapy were similar among schizophrenia $(56.8 \%)$ and adult bipolar disorder (56.3\%) patients. As in schizophrenia population, non-adherence is a 
major problem with bipolar disorder patients (Keck, Jr., McElroy, Strakowski, Bourne, \& West, 1997). Prevalence of substance abuse disorder and denial of disease condition have been cited as the reasons for non-adherence among bipolar disorder patients (Keck, Jr. et al., 1997; Lacro et al., 2002; Scott \& Pope, 2002). Though adherence to antipsychotics has not been studied in previous studies, the rate of partial or total nonadherence with mood stabilizers was reported to be about $51 \%$ in bipolar disorder population (Keck, Jr. et al., 1997). About one in three patients with bipolar disorder take less than 30 percent of their medication (Scott et al., 2002). However, the rate of interrupted therapy was much higher among bipolar disorder patients less than 18 years of age. Since antipsychotics were not approved by the FDA for bipolar disorder at the time of study, it is possible that physicians were wary of using antipsychotics for extended periods in children and were using them for short periods to control symptoms.

\section{Results for the research objective 4}

For schizophrenia and bipolar disorder patients, determine the gaps between the refills

of antipsychotics.

\section{$\underline{\text { Schizophrenia }}$}

Table 6 describes treatment gaps among schizophrenia patients in terms of the longest continuous gap between refills of antipsychotics for each patient. About 27.5\% of patients had no gaps greater than 15 days between refills of antipsychotic therapy. About $60.9 \%$ of patients had at least 1 gap of greater than 30 days. About $30.4 \%$ of patients had at least 1 gap of greater than 90 days between refills of antipsychotics. Average duration of the longest gap was $72.2( \pm 75.6)$ days. $\underline{\text { Bipolar Disorder }}$ 
Table 7 describes treatment gaps among schizophrenia patients in terms of the longest continuous gap between refills of antipsychotics for each patient. About 26.8\% of 18 years and older bipolar disorder patients and about $37.2 \%$ of less than 18 years old bipolar disorder patients had no gaps greater than 15 days between refills of antipsychotic therapy. About $23.8 \%$ of 18 years and older bipolar disorder patients and about $17.6 \%$ of less than 18 years old bipolar disorder patients had at least 1 gap of greater than 90 days between refills of antipsychotics. Average duration of the longest gap was $64.1( \pm 71.3)$ days for patients 18 years or older and $48( \pm 63.9)$ days for patients less than 18 years of age. 
Table 6: Study of antipsychotic treatment gaps among schizophrenia patients (18 years and above)

\begin{tabular}{ll}
\multicolumn{1}{c}{ Duration of gap * in days } & $\boldsymbol{N}=\mathbf{1 , 0 3 6}$ \\
\hline $\begin{array}{l}\text { Mean }( \pm \underline{\text { std }}) \\
\text { Median }\end{array}$ & $72.2( \pm 75.6)$ \\
Up to or equal to 15 days & $285(27.5 \%)$ \\
Greater than 15 days to 30 days & $121(11.7 \%)$ \\
Greater than 30 days to 60 days & $188(18.2 \%)$ \\
Greater than 60 days to 90 days & $127(12.3 \%)$ \\
Greater than 90 days & $315(30.4 \%)$ \\
\hline
\end{tabular}

* The longest continuous gap between refills for each patient 
Table 7: Study of antipsychotic treatment gaps among bipolar disorder patients

\begin{tabular}{lccc}
\hline $\begin{array}{c}\text { Duration of gap* in } \\
\text { days }\end{array}$ & $\begin{array}{c}\mathbf{1 8} \text { years and above } \\
\boldsymbol{n}=\mathbf{6 8 4}\end{array}$ & $\begin{array}{c}\text { Less than 18 years } \\
\boldsymbol{n}=\mathbf{1 4 8}\end{array}$ & $\begin{array}{c}\text { Total } \\
\mathbf{N}=\mathbf{8 3 2}\end{array}$ \\
\hline $\begin{array}{l}\text { Mean }( \pm \mathrm{std}) \\
\text { Median }\end{array}$ & $\begin{array}{c}64.1( \pm 71.3) \\
37.0\end{array}$ & $\begin{array}{c}48.0( \pm 63.9) \\
26.0\end{array}$ & $\begin{array}{c}61.2( \pm 70.3) \\
33.0\end{array}$ \\
$\begin{array}{l}\text { Up to or equal to } 15 \\
\text { days }\end{array}$ & $183(26.8 \%)$ & $55(37.2 \%)$ & $194(28.6 \%)$ \\
$\begin{array}{l}\text { Greater than } 15 \text { days to } \\
30 \text { days }\end{array}$ & $117(17.1 \%)$ & $31(20.9 \%)$ & $148(17.8 \%)$ \\
$\begin{array}{l}\text { Greater than } 30 \text { days to } \\
60 \text { days } \\
\text { Greater than } 60 \text { days to }\end{array}$ & $137(20.0 \%)$ & $27(18.2 \%)$ & $164(19.7 \%)$ \\
90 days & $84(12.2 \%)$ & $9(6.08 \%)$ & $93(11.2 \%)$ \\
Greater than 90 days & $163(23.8 \%)$ & $26(17.6 \%)$ & $189(22.7 \%)$ \\
\hline
\end{tabular}

* The longest continuous gap between refills for each patient 


\section{Discussion for the research objective 4}

$\underline{\text { Schizophrenia }}$

Gaps in the antipsychotic therapy are a measure of compliance or adherence to therapy. Poor adherence to antipsychotic therapy among schizophrenia patients can be attributed to the nature of disease as well as side-effects to the antipsychotic therapy. Large gaps in antipsychotic therapy as found in our study have also been observed in other published studies. McCombs and colleagues have noted "drug holidays" among schizophrenia patients with only $11.6 \%$ of patients received uninterrupted antipsychotic therapy. About $30.7 \%$ of a treated sample of 2,010 patients had gaps larger than 90 days

(McCombs, Nichol, Stimmel, Shi, \& Smith, 1999a). Mojtabai and colleagues report that about $51 \%$ of patients discharged from hospital had gaps of greater than 30 days in antipsychotic treatment within a year(Mojtabai et al., 2002). Weiden and colleagues found gaps of greater than 30 days among $25.97 \%$ of patients in California Medicaid (Weiden, Kozma, Grogg, \& Locklear, 2004).

$\underline{\text { Bipolar Disorder }}$

Our study found large gaps in antipsychotic therapy were present among bipolar disorder patients as well as schizophrenia patients. Though few studies have reported the problem of non-adherence to mood stabilizer therapy in bipolar disorder patients, gaps in the therapy or adherence to antipsychotic therapy has not been explored. Gaps in antipsychotic therapy among bipolar disorder patients may be due to drug side-effects, patient denial of illness and alcohol and substance abuse (Keck, Jr. et al., 1997; Lacro et al., 2002; Scott et al., 2002). 


\section{Results for research objective 5}

For schizophrenia and bipolar disorder patients, determine predictors of different utilization patterns of antipsychotics

$\underline{\text { Schizophrenia }}$

Table 8 presents the results of the multinomial logistic model predicting factors associated with patterns of antipsychotic use among schizophrenia patients. Alcohol and substance abuse, and use of typical antipsychotics as index prescription showed significant association with all patterns of antipsychotic use. In addition, Schizophrenia subtypes, diagnosis of major depression, prescription of mood stabilizers, antidepressants and antipsychotics in the pre-index period were also significantly associated with certain patterns of antipsychotic use.

Patients having diagnosis of alcohol and substance abuse were almost 2 times more likely to show either interrupted therapy, switching or polytherapy pattern than continuous therapy pattern. Patients showing mixed diagnosis of schizophrenia and schizoaffective disorders were almost 2 times more likely to show continuous therapy rather than switching or polytherapy. Patients having major depression were 2.3 times more likely to receive polytherapy rather than continuous monotherapy.

Compared to patients initiated on quetiapine, patients initiated on typical antipsychotic were about 5 times more likely to receive interrupted antipsychotic therapy, about 1.1 times more likely to switch, and about 5.6 times more likely to receive polytherapy. 
Table 8: Multinomial logistic regression model determining predictors of pattern of antipsychotic use among schizophrenia patients (first-stage sample selection model)

\begin{tabular}{ccc}
\hline $\begin{array}{c}\text { Interrupted } v s . \\
\text { Adherent }\end{array}$ & $\begin{array}{c}\text { Switching } v \text { s. } \\
\text { Adherent }\end{array}$ & $\begin{array}{c}\text { Polytherapy vs. } \\
\text { Adherent }\end{array}$ \\
\hline
\end{tabular}

Coefficient t-ratio Coefficient t-ratio Coefficient t-ratio

\section{Demographic characteristics}

\begin{tabular}{|c|c|c|c|c|c|c|}
\hline Age (in years) & -0.009 & -1.192 & -0.021 & 1.926 & 0.001 & 0.15 \\
\hline Males (ref: females) & 0.008 & 0.627 & 0.751 & 0.047 & 0.005 & 0.246 \\
\hline $\begin{array}{l}\text { Whites (ref: non- } \\
\text { whites) }\end{array}$ & 0.007 & 0.024 & 0.293 & 0.675 & -0.111 & -0.273 \\
\hline Metro (ref: non-metro) & 0.068 & 0.291 & -0.424 & 1.408 & -0.215 & -0.697 \\
\hline \multicolumn{7}{|c|}{ Prescribing physician type } \\
\hline Psychiatric prescriber & -0.001 & -1.871 & -0.008 & -1.647 & -0.005 & -1.501 \\
\hline \multicolumn{7}{|c|}{ Schizophrenia subtype (ref: only schizophrenia) } \\
\hline $\begin{array}{l}\text { Schizoaffective } \\
\text { disorder }\end{array}$ & 0.219 & 0.867 & 0.0844 & 0.268 & -0.384 & -1.127 \\
\hline $\begin{array}{l}\text { Both schizoaffective } \\
\text { disorder and } \\
\text { schizophrenia }\end{array}$ & 0.162 & 0.646 & -0.777 & $-2.309^{*}$ & -0.965 & $-2.647^{\star}$ \\
\hline \multicolumn{7}{|c|}{ Pre-index co-morbidities } \\
\hline Diabetes & 0.493 & 1.464 & 0.349 & 0.793 & -0.183 & -0.357 \\
\hline Hyperlipidemia & 0.021 & 0.057 & -0.349 & -0.65 & 0.099 & 0.162 \\
\hline Hypertension & 0.122 & 0.454 & 0.331 & 0.949 & -0.489 & -1.113 \\
\hline $\begin{array}{l}\text { Alcohol and substance } \\
\text { abuse }\end{array}$ & 0.686 & $2.676^{*}$ & 0.701 & $2.193^{*}$ & 0.873 & $2.614^{*}$ \\
\hline Bipolar disorder & 0.547 & 1.931 & 0.313 & 0.873 & 0.293 & 0.724 \\
\hline Major depression & 0.195 & 0.749 & 0.437 & 1.348 & 0.834 & $2.382^{*}$ \\
\hline Anxiety disorder & -0.164 & -0.639 & -0.178 & -0.532 & -0.305 & -0.802 \\
\hline Personality disorder & -0.160 & -0.532 & 0.086 & 0.232 & -0.120 & -0.279 \\
\hline $\begin{array}{l}\text { Non-specified } \\
\text { psychosis }\end{array}$ & -0.647 & -1.497 & -1.382 & -1.923 & -1.182 & -1.731 \\
\hline $\begin{array}{l}\text { Other affective } \\
\text { psychosis }\end{array}$ & -0.797 & -1.381 & -0.461 & -0.597 & -1.312 & -1.111 \\
\hline
\end{tabular}


Table 8: Multinomial logistic regression model determining predictors of pattern of antipsychotic use among schizophrenia patients (first-stage sample selection model) (contd.)

\begin{tabular}{|c|c|c|c|c|c|c|}
\hline & \multicolumn{2}{|c|}{$\begin{array}{c}\text { Interrupted vs. } \\
\text { Adherent }\end{array}$} & \multicolumn{2}{|c|}{$\begin{array}{l}\text { Switching vs. } \\
\text { Adherent }\end{array}$} & \multicolumn{2}{|c|}{$\begin{array}{c}\text { Polytherapy vs. } \\
\text { Adherent }\end{array}$} \\
\hline & Coefficient & t-ratio & Coefficient & t-ratio & Coefficient & t-ratio \\
\hline $\begin{array}{l}\text { Attention deficit } \\
\text { disorders }\end{array}$ & -0.669 & -1.238 & -0.217 & -0.322 & -0.056 & -0.072 \\
\hline $\begin{array}{l}\text { Mild to moderate } \\
\text { depression }\end{array}$ & 0.240 & 0.786 & 0.351 & 0.923 & 0.143 & 0.325 \\
\hline \multicolumn{7}{|c|}{ Pre-index healthcare utilization and cost } \\
\hline $\begin{array}{l}\text { Number of pre-index } \\
\text { mental-health related } \\
\text { ER visits/ } \\
\text { hospitalizations }\end{array}$ & 0.005 & 1.558 & -0.005 & -1.066 & -0.002 & -0.476 \\
\hline $\begin{array}{l}\text { Number of pre-index } \\
\text { mental-health related } \\
\text { physician visits }\end{array}$ & -0.129 & -1.345 & 0.084 & 0.856 & 0.062 & 0.59 \\
\hline $\begin{array}{l}\text { Number of } \\
\text { psychotherapy visits }\end{array}$ & 0.008 & 0.398 & -0.038 & -0.689 & 0.018 & 0.645 \\
\hline $\begin{array}{l}\text { Number of medicatior } \\
\text { management visits }\end{array}$ & 0.009 & 0.3 & -0.043 & -1.012 & -0.037 & -0.784 \\
\hline \multicolumn{7}{|c|}{ Year of index prescription (ref: 1999) } \\
\hline - 2000 & 0.209 & 0.868 & 0.217 & 0.675 & -0.251 & -0.684 \\
\hline - 2001 & 0.190 & 0.71 & 0.105 & 0.286 & -0.023 & -0.058 \\
\hline \multicolumn{7}{|c|}{ Pre-index period psychiatric medication use ( days of supply) } \\
\hline Mood stabilizers & -0.341 & $-2.542^{*}$ & -0.869 & -0.107 & -0.000 & -0.128 \\
\hline Anticholinergics & -0.017 & -1.386 & -0.003 & -0.584 & 0.002 & 0.303 \\
\hline $\begin{array}{l}\text { Anxiolytics/hypnotics } \\
\text { sedatives }\end{array}$ & 0.004 & 0.25 & 0.001 & 1.004 & 0.007 & 0.338 \\
\hline Antipsychotics & -0.125 & $-2.314^{*}$ & -0.003 & -1.007 & -0.006 & -0.227 \\
\hline Antidepressants & -0.003 & -0.489 & 0.322 & $2.656^{*}$ & -0.003 & -0.332 \\
\hline Benzodiazepines & -0.003 & -0.422 & -0.001 & -1.27 & 0.001 & 1.597 \\
\hline Antiparkinsons & 0.006 & 0.245 & 0.003 & 1.08 & 0.005 & 0.142 \\
\hline
\end{tabular}


Table 8: Multinomial logistic regression model determining predictors of pattern of antipsychotic use among schizophrenia patients (first-stage sample selection model) (contd.)

\begin{tabular}{lcccccc}
\hline & $\begin{array}{c}\text { Interrupted vs. } \\
\text { Adherent }\end{array}$ & $\begin{array}{c}\text { Switching vs. } \\
\text { Adherent }\end{array}$ & \multicolumn{2}{c}{$\begin{array}{c}\text { Polytherapy vs. } \\
\text { Adherent }\end{array}$} \\
& Coefficient & t-ratio & Coefficient & t-ratio & Coefficient & t-ratio \\
\hline $\begin{array}{l}\text { Index prescription (ref: quetiapine) } \\
\text { Risperidone }\end{array}$ & 0.309 & 1.09 & 0.144 & 0.364 & 0.819 & 1.580 \\
Olanzapine & 0.025 & 0.102 & 0.218 & 0.422 & 0.543 & 1.130 \\
Typicals & 1.628 & $5.112^{*}$ & 0.108 & $2.567^{*}$ & 1.730 & $3.289^{*}$ \\
\hline
\end{tabular}

*significant at $\mathrm{p}<0.05$

Model fit statistics:

Pseudo $R$-square $=13.1 \%,-2$ Log Likelihood $=1034.62 ; \chi^{2}=314.49 ; p=0.00$ 


\section{$\underline{\text { Bipolar Disorder }}$}

Table 9 presents the results of the multinomial logistic model predicting factors associated with patterns of antipsychotic use among bipolar disorder patients. Compared to bipolar disorder patients who were less than 18 years of age, bipolar disorder patients who were 18 years or older were almost 2 times less likely to have adherent index antipsychotic therapy. Patients having psychiatric prescriber for the index prescription were 1.22 times less likely to have interrupted therapy. Patients having diagnosis of alcohol and substance abuse were almost 2.3 times more likely to show interrupted therapy, 2.8 times more likely to switch, and 2.7 times more likely to have polytherapy pattern than continuous therapy pattern. Patients using mood stabilizers in the pre-index period were 1.3 times more likely to have adherent antipsychotic therapy. Patients initiated on typicals were 1.86 times more likely to have interrupted antipsychotic therapy.

Patients having mixed bipolar disorder patients were almost 2 times less likely to switch. Patients who have used antidepressants in the pre-index period were almost 1.2 times more likely to switch index antipsychotic therapy. Patients who have used typical antipsychotics were almost 1.13 times more likely to switch index antipsychotic therapy.

Patients who have major depression are 2.5 times more likely to receive polytherapy. Patients who have anxiety are 2.8 times less likely to receive polytherapy. Patients receiving typical antipsychotics were 3.3 times more likely to receive polytherapy. 
Table 9: Multinomial logistic regression model determining predictors of pattern of antipsychotic use among bipolar disorder patients (first-stage sample selection model)

\begin{tabular}{|c|c|c|c|c|c|c|}
\hline & \multicolumn{2}{|c|}{$\begin{array}{c}\text { Interrupted vs. } \\
\text { Adherent } \\
\end{array}$} & \multicolumn{2}{|c|}{$\begin{array}{c}\text { Switching vs. } \\
\text { Adherent }\end{array}$} & \multicolumn{2}{|c|}{$\begin{array}{c}\text { Polytherapy vs. } \\
\text { Adherent }\end{array}$} \\
\hline & Coefficient & t-ratio & Coefficient & t-ratio & Coefficient & t-ratio \\
\hline \multicolumn{7}{|c|}{ Demographic characteristics } \\
\hline Age (in years) & -0.007 & -0.730 & -0.019 & -1.53 & 0.014 & 0.956 \\
\hline $\begin{array}{l}18 \text { years or above (ref: } \\
\text { less than } 18 \text { years) }\end{array}$ & 0.719 & $2.371^{*}$ & -0.338 & -0.845 & -0.810 & -1.942 \\
\hline Males (ref: females) & 0.002 & 1.417 & 0.001 & 0.554 & 0.108 & 0.379 \\
\hline $\begin{array}{l}\text { Whites (ref: non- } \\
\text { whites) }\end{array}$ & -0.041 & -0.117 & 0.224 & 0.462 & 0.200 & 0.356 \\
\hline $\begin{array}{l}\text { Metro (ref: non- } \\
\text { metro) }\end{array}$ & 0.146 & 0.541 & -0.347 & -1.007 & -0.232 & -0.596 \\
\hline \multicolumn{7}{|c|}{ Prescribing physician type } \\
\hline Psychiatric prescriber & -0.200 & $-2.212^{*}$ & -0.001 & -1.036 & -0.001 & -1.563 \\
\hline \multicolumn{7}{|c|}{ Bipolar Subtype (ref: bipolar disorder I) } \\
\hline Bipolar disorder II & 0.318 & 1.095 & 0.181 & 0.509 & -0.144 & -0.346 \\
\hline Mixed & 0.260 & 0.923 & -0.742 & $-2.015^{*}$ & -0.769 & -1.824 \\
\hline \multicolumn{7}{|c|}{ Pre-index co-morbidities } \\
\hline Diabetes & 0.409 & 1.124 & 0.259 & 0.533 & -0.782 & -1.094 \\
\hline Hyperlipidemia & -0.002 & -0.005 & -0.616 & -1.005 & 0.153 & 0.199 \\
\hline Hypertension & 0.144 & 0.496 & 0.331 & 0.883 & -0.315 & -0.608 \\
\hline $\begin{array}{l}\text { Alcohol and substance } \\
\text { abuse }\end{array}$ & 0.877 & $2.916^{*}$ & 1.033 & $2.867^{*}$ & 1.010 & $2.380^{*}$ \\
\hline Bipolar disorder & 0.520 & 1.656 & 0.060 & 0.147 & 0.076 & 0.148 \\
\hline Major depression & 0.356 & 1.234 & 0.529 & 1.49 & 0.925 & $2.151^{*}$ \\
\hline Anxiety disorder & -0.366 & -1.273 & -0.190 & -0.517 & -1.058 & $-1.966^{*}$ \\
\hline Personality disorder & -0.091 & -0.273 & 0.066 & 0.16 & -0.227 & -0.416 \\
\hline $\begin{array}{l}\text { Non-specified } \\
\text { psychosis }\end{array}$ & -0.634 & -1.248 & -1.175 & -1.501 & -0.378 & -0.506 \\
\hline $\begin{array}{l}\text { Other affective } \\
\text { psychosis }\end{array}$ & -0.788 & -1.194 & 0.003 & 0.003 & -28.058 & 0.000 \\
\hline
\end{tabular}


Table 9: Multinomial logistic regression model determining predictors of pattern of antipsychotic use among bipolar disorder patients (first-stage sample selection model) (contd.)

\begin{tabular}{|c|c|c|c|c|c|c|}
\hline & \multicolumn{2}{|c|}{$\begin{array}{c}\text { Interrupted vs. } \\
\text { Adherent } \\
\end{array}$} & \multicolumn{2}{|c|}{$\begin{array}{c}\text { Switching vs. } \\
\text { Adherent }\end{array}$} & \multicolumn{2}{|c|}{$\begin{array}{c}\text { Polytherapy vs. } \\
\text { Adherent }\end{array}$} \\
\hline & Coefficient & t-ratio & Coefficient & t-ratio & Coefficient & t-ratio \\
\hline $\begin{array}{l}\text { Attention deficit } \\
\text { disorders }\end{array}$ & -0.682 & -1.237 & -0.285 & -0.415 & 0.133 & 0.162 \\
\hline $\begin{array}{l}\text { Mild to moderate } \\
\text { depression }\end{array}$ & 0.148 & 0.448 & 0.240 & 0.58 & -0.015 & -0.028 \\
\hline $\begin{array}{l}\text { Number of pre-index } \\
\text { mental-health related } \\
\text { ER visits/ } \\
\text { hospitalizations }\end{array}$ & 0.001 & 1.534 & -0.001 & -1.18 & 0.000 & -0.224 \\
\hline $\begin{array}{l}\text { Number of pre-index } \\
\text { mental-health related } \\
\text { physician visits }\end{array}$ & -0.095 & -0.886 & 0.105 & 0.958 & 0.124 & 1.040 \\
\hline $\begin{array}{l}\text { Number of } \\
\text { psychotherapy visits }\end{array}$ & -0.019 & -0.764 & -0.048 & -0.789 & -0.105 & -0.244 \\
\hline $\begin{array}{l}\text { Number of medication } \\
\text { management visits }\end{array}$ & 0.050 & 1.461 & -0.072 & -1.408 & -0.177 & -0.357 \\
\hline \multicolumn{7}{|c|}{ Year of index prescription (ref: 1999) } \\
\hline - 2000 & 0.056 & 0.213 & 0.123 & 0.354 & 0.000 & -0.414 \\
\hline - 2001 & 0.025 & 0.084 & -0.016 & -0.041 & 0.006 & 0.928 \\
\hline \multicolumn{7}{|c|}{ Pre-index period psychiatric medication use ( days of supply) } \\
\hline Mood stabilizers & -0.320 & $-3.041^{*}$ & 0.000 & 0.137 & 0.003 & 1.250 \\
\hline Anticholinergics & -0.007 & -1.298 & -0.005 & -0.708 & 0.001 & 0.248 \\
\hline $\begin{array}{l}\text { Anxiolytics/hypnotics/ } \\
\text { sedatives }\end{array}$ & 0.001 & 0.464 & 0.002 & 1.04 & -0.002 & -1.787 \\
\hline Antipsychotics & -0.106 & $-2.293^{*}$ & -0.005 & -1.327 & 0.001 & 1.053 \\
\hline Antidepressants & -0.001 & -1.219 & 0.202 & $2.442^{*}$ & 0.000 & -0.028 \\
\hline Benzodiazepines & -0.656 & -0.079 & -0.001 & -1.266 & 0.017 & 0.575 \\
\hline Antiparkinsons & -0.001 & -0.207 & -0.671 & -0.016 & -0.012 & -0.208 \\
\hline
\end{tabular}


Table 9: Multinomial logistic regression model determining predictors of pattern of antipsychotic use among bipolar disorder patients (first-stage sample selection model) (contd.)

\begin{tabular}{lcccccc}
\hline & $\begin{array}{c}\text { Interrupted vs. } \\
\text { Adherent } \\
\text { Coefficient }\end{array}$ & $\begin{array}{c}\text { t-ratio } \\
\text { Coefficient }\end{array}$ & $\begin{array}{c}\text { Adherent } \\
\text { Cofing vs. }\end{array}$ & $\begin{array}{c}\text { Polytherapy vs. } \\
\text { Adherent }\end{array}$ \\
\hline Index prescription (ref: quetiapine) & & & & & t-ratio \\
Risperidone & -0.001 & -1.589 & -0.001 & -1.751 & -0.002 & -1.237 \\
Olanzapine & -0.001 & -1.719 & 0.000 & -1.368 & -0.002 & -1.536 \\
Typicals & 0.620 & $3.132^{*}$ & 0.13 & $2.461^{*}$ & 1.210 & $2.075^{*}$ \\
\hline
\end{tabular}

* significant at $\mathrm{p}<0.05$

Model fit statistics:

Pseudo R-square $=13.67 \%$

-2 Log Likelihood $=801.14 ; \chi^{2=} 253.90 ; p=0.00$ 


\section{Discussion for research objective 5}

\section{$\underline{\text { Schizophrenia }}$}

Alcohol and substance abuse is a common co morbidity among schizophrenia patients (APA, 1997). Our study found that alcohol and substance abuse was a significant predictor of interrupted therapy, switching, and polytherapy. Other studies have also reported association of substance abuse with non-adherence with medications (Hudson et al., 2004; Hunt, Bergen, \& Bashir, 2002; Kashner et al., 1991; Kamali et al., 2001). A study of barriers to medication adherence among schizophrenia patients reported patients with substance and alcohol abuse were 3.24 times more likely to be non-compliant with the medications (Hudson et al., 2004). As management of disease condition may be difficult in these patients, physicians may resort to switching or polytherapy to treat patients. As use of alcohol and drugs are strictly contraindicated with use of psychotropic medications, the patients may choose to stop taking medications and take alcohol or drugs.

Patients with mixed diagnosis of both schizophrenia and schizoaffective disorder may be showing range of symptoms. Physicians may be attempting to control the symptoms by changing or augmenting antipsychotic therapy. According to our study results, patients with major depression were 2.3 times more likely to receive polytherapy. A study by McCombs and colleagues also reported about 1.9 times greater likelihood of switching or polytherapy among schizophrenia patients with diagnosis of major depression (McCombs et al., 1999a). Polytherapy may have been used in these patients 
to manage greater exacerbation of disease symptoms due to presence of an additional mental illness.

Compared to patients initiated on quetiapine, patients initiated on typical antipsychotic had a high likelihood of interrupted antipsychotic therapy, switching and polytherapy. Typical antipsychotics have been associated with poor adherence due to disabling adverse effects such as extrapyramidal symptoms (EPS) (Van Putten, 1974). Studies have reported poorer adherence rates with typical antipsychotics $(50.1 \%)$ compared to atypical antipsychotics (54.9\%) (Dolder, Lacro, Dunn, \& Jeste, 2002). Menzin and colleagues found that schizophrenia patients on atypical antipsychotics in the California Medicaid population were less likely to switch $(\mathrm{OR}=0.37)$ than typical antipsychotics in 1997(Menzin et al., 2003). Compared with quetiapine, olanzapine and risperidone did not show any significant difference in the likelihood of interrupted therapy, switching, and polytherapy. Though few studies have reported better persistence with index antipsychotic for olanzapine with respect to risperidone (Gibson et al., 2004), no studies have compared the typical and atypical antipsychotics in terms of continuous, interrupted, switching and polytherapy patterns.

\section{$\underline{\text { Bipolar Disorder }}$}

As among schizophrenia patients, we found alcohol and substance abuse was a significant predictor of interrupted therapy, switching or polytherapy among bipolar patients. Prevalence of alcohol and substance abuse is about $40 \%$ to $60 \%$ among bipolar disorder patients (Salloum \& Thase, 2000). Other studies have also reported association of alcohol and substance abuse with medication non-adherence, especially in schizophrenia population (Keck, Jr. et al., 1997). 
Patterns of antipsychotic therapy are relatively less studied among bipolar disorder patients. Common factors associated with non-adherence to mood-stabilizers medications among bipolar disorder are mania, personality disorders, alcohol and substance abuse, and denial of disorder or need for treatment (Keck, Jr. et al., 1997; Loosbrock et al., 2003; Maarbjerg, Aagaard, \& Vestergaard, 1988; Schumann, Lenz, Berghofer, \& Muller-Oerlinghausen, 1999). In our study, factors such as disease subtype, use of mood stabilizers, antidepressants, and typical antipsychotics, alcohol and substance abuse were common predictors of patterns of antipsychotic use among bipolar and schizophrenia patients.

The significant association of use of typical antipsychotics with interrupted therapy and switching may be due to side-effects. Use of mood-stabilizers was associated with decreased likelihood of receiving interrupted therapy. Presence of major depression was linked with greater likelihood of receiving polytherapy. Major depression was also associated with polytherapy among schizophrenia patients in our study as well as in the McCombs study (McCombs et al., 1999a).

\section{Results for research objective 6}

For schizophrenia and bipolar disorder patients, determine the relationship between utilization pattern of antipsychotics and total health-related healthcare costs.

The objective was to determine the effect of pattern of antipsychotic use on total healthcare costs among schizophrenia and bipolar patients. The results of univariate analysis show that the post-index total healthcare costs was significantly less for the continuous therapy group compared to the interrupted, switch and polytherapy group. Multivariate analysis was conducted to test this difference after controlling for patient 
demographics, disease severity, co morbidities and prior utilization of healthcare services. An important step of this analysis was to adjust for the sample selection bias between patients showing different patterns of antipsychotic utilization. In the first step of the analysis, a multinomial logistic regression analysis was carried out using various explanatory variables such as patient demographics, co morbidities and healthcare utilization variables. This model was same as the model provided in the table 8 for schizophrenia patients and table 9 for bipolar disorder patients. This model allowed for calculation of Inverse Mills Ratio (IMR) to adjust for sample selection bias in the second step regression models. Individual contrasts were carried out to obtain IMR for continuous vs. interrupted therapy, continuous vs. switching therapy, and continuous vs. polytherapy.

In the second step of the analysis, multivariate regression models were used to investigate association of different patterns of antipsychotic use with total healthcare costs. Three semi-log OLS regressions were developed to compare costs associated with interrupted therapy, switching and polytherapy with adherent therapy. IMRs calculated from the first stage regression models were added as a covariate in the second stage regression models to adjust and test for sample selection bias. The models were further tested for appropriate specifications. It is possible that multicollinarity resulting due to inclusion of IMR in the model may be inflating the standard errors. Therefore, a semi$\log$ OLS model was developed without including the IMR and the output was compared with the outputs of regression models that included IMR.

The univariate analysis for total and mental healthcare costs for schizophrenia patients are presented in table 10. The second stage regression models for total and 
mental healthcare costs for schizophrenia patients are presented in the tables 11 and 12, respectively.

The univariate analysis for total and mental healthcare costs for bipolar disorder patients are presented in table 13. The second stage regression models for total and mental healthcare costs for bipolar disorder patients are presented in the tables 14 and 15, respectively.

\section{$\underline{\text { Schizophrenia }}$}

The results of univariate analysis presented in table 10 show that the post-index total healthcare costs was significantly less for the continuous therapy group $(\$ 19,960 \pm$ $\$ 32,312)$ compared to the switching $(\$ 21,595 \pm \$ 24,595)$ and polytherapy $(\$ 27,741 \pm$ $35,334)$ group. The multivariate analyses of total costs are presented in tables 11a-11c. The results of multivariate analysis presented in table 11a show that patients receiving interrupted index antipsychotic therapy incur $7.89 \%$ higher total healthcare costs than patients receiving continuous antipsychotic therapy. After controlling for confounding variables, there were no significant differences among patients receiving continuous antipsychotic therapy and patients switching from index antipsychotic therapy (table 11b). Table $11 \mathrm{c}$ shows that patients receiving polytherapy incur $28.40 \%$ higher total costs than patients receiving continuous index antipsychotic therapy. Also, IMR was not significant in all the models indicating possible absence of selection bias between patients on different patterns of antipsychotic use cohorts.

$\underline{\text { Bipolar disorder }}$

The results of univariate analysis presented in table 13 show that the post-index total healthcare costs was significantly less for the continuous therapy group $(\$ 18,383 \pm$ 
$\$ 30,283)$ as compared to the interrupted $(\$ 20,486 \pm \$ 21,513)$, switching $(\$ 20,104 \pm$

$\$ 23,116)$ and polytherapy $(\$ 26,972 \pm 32,885)$ groups. The results of multivariate

analysis presented in table 14a show that patients receiving interrupted index

antipsychotic therapy incur $1.5 \%$ higher total healthcare costs than patients receiving continuous antipsychotic therapy. Table $14 \mathrm{~b}$ shows that patients switching from index antipsychotic to monotherapy with another antipsychotic incur $17.0 \%$ higher total costs than patients receiving continuous index antipsychotic therapy. Table $14 \mathrm{c}$ shows that patients receiving polytherapy incur $37.71 \%$ higher total costs than patients receiving continuous index antipsychotic therapy.

\section{Results for research objective 7}

For schizophrenia and bipolar disorder patients, determine the relationship between utilization pattern of antipsychotics and mental health care utilization and costs.

\section{$\underline{\text { Schizophrenia }}$}

The results of univariate analysis presented in table 10 show that the post-index mental healthcare costs was significantly less for the continuous therapy group $(\$ 18,383$ $\pm \$ 30,283)$ compared to the interrupted $(\$ 12,482 \pm \$ 24,195)$, switching $(\$ 17,890 \pm$ $\$ 18,330)$ and polytherapy $(\$ 21,006 \pm \$ 35,334)$ groups. The multivariate results in table 12a show that after controlling for confounding variables, patients receiving interrupted index antipsychotic therapy incur 3.44\% higher mental healthcare costs than patients receiving continuous antipsychotic therapy. After controlling for confounding variables, there were no significant differences among patients receiving continuous antipsychotic therapy and patients switching from index antipsychotic therapy (table 12b). Table 12c 
shows that patients receiving polytherapy incur $12.74 \%$ higher mental health care costs than patients receiving continuous index antipsychotic therapy.

\section{$\underline{\text { Bipolar }}$}

The results of univariate analysis presented in table 13 show that the post-index mental healthcare costs was significantly less for the continuous therapy group $(\$ 18,383$ $\pm \$ 30,283)$ as compared to the interrupted $(\$ 11,134 \pm \$ 21,901)$, switching $(\$ 17,890 \pm$ $\$ 18,330)$ and polytherapy $(\$ 16,470 \pm \$ 15,258)$ group. The multivariate results in table 15a show that after controlling for confounding variables, patients receiving interrupted index antipsychotic therapy incur $7.03 \%$ higher total healthcare costs than patients receiving continuous antipsychotic therapy. After controlling for confounding variables, there were no significant differences among patients receiving continuous antipsychotic therapy and patients switching from index antipsychotic therapy (table 15b). Table 15c shows that patients receiving polytherapy incur $16.18 \%$ higher total costs than patients receiving continuous index antipsychotic therapy.

\section{Discussion for research objective 6 and 7}

\section{$\underline{\text { Schizophrenia and bipolar disorder }}$}

Our results show that significant increase in total and mental costs is associated with different patterns of antipsychotic use. Other studies have also found that nonadherent patients are more costly than adherent patients. A study by Knapp and colleagues conducted among adults living in institutions in the UK reported that nonadherent patients incurred an additional $\$ 5000$ in total costs than adherent patients (Knapp, King, Pugner, \& Lapuerta, 2004). Eaddy and colleagues report 54.5\% increase in inpatient costs among partially adherent patients (Eaddy et al., 2005). Gilmer and 
colleagues found that non-adherent patients incur almost 3 times higher inpatient cost than adherent patients in a California Medicaid population. Risk of hospitalization has also been shown to increase with increasing gaps in therapy (Weiden et al., 2004; Gilmer et al., 2004). Literature on association of pattern of medication use with healthcare costs and utilization are relatively rare in bipolar disorder. Studies have shown that the risk of relapse resulting in hospitalizations increases after discontinuing lithium treatment in bipolar disorder patients (Schou, 1997; Suppes, Baldessarini, Faedda, \& Tohen, 1991). Our study demonstrates that patients with non-adherent or interrupted antipsychotic therapy are more likely to incur higher costs.

Our results show that switching from index antipsychotic to another antipsychotic was associated with significant increase in cost for bipolar disorder patients but not for schizophrenia patients. Other studies in literature have suggested that switching can increase costs as it usually occurs when a patient fails the previous treatment or experiences severe side-effects (McCombs et al., 2000; Loosbrock et al., 2003). McCombs and colleagues reported that antipsychotic switching can increase the annual total cost by $\$ 9,719$ (McCombs et al., 2000). However, they combined the cost of switching to antipsychotic monotherapy or polytherapy in their analysis which could have overestimated the increase in total costs. Loosberg and colleagues also found an increase of $\$ 4,706$ associated switching antipsychotics(Loosbrock et al., 2003). As the data used in above two studies is prior to 1997 and a large proportion of the study population was on typical antipsychotics, it is likely that increase in cost was mainly due to switching from typical to atypical antipsychotics, which are more costly. Switching antipsychotics is advocated by the treatment guidelines when patient fails to respond or 
stabilize on current medications and may lead to finding the appropriate medication regimen for the patients.

Significantly greater increase in total and mental costs was associated with polytherapy for schizophrenia and bipolar disorder patients. Results are similar to those observed in the Loosbrock study that evaluated total costs differences between schizophrenia patients on polytherapy and monotherapy. The study reported that schizophrenia patients on polytherapy incurred an additional $\$ 4,244$ in total costs as compared to schizophrenia patients on monotherapy. However, it should be noted that in our study comparisons are being made between polytherapy and adherent or continuous monotherapy.

Antipsychotic polytherapy has been associated with increased risk of adverse effects and hospitalization (Centorrino et al., 2004). Utilization of anticholinergics among patients on antipsychotics was significantly higher as compared to monotherapy (Procyshyn et al., 2001). The cost of adverse reactions as well as that of utilization of additional drug may be driving the cost of polytherapy higher. It is also likely that the patients on polytherapy are more severe or treatment refractory and are utilizing more healthcare services. However, results were significant after controlling for the severity and selection bias for these patients using pre-index comorbidities and utilization-related factors. 
Table 10: Total and mental healthcare costs associated with the different antipsychotic utilization patterns among schizophrenia patients

\begin{tabular}{|c|c|c|c|c|}
\hline & $\begin{array}{c}\text { Continuous } \\
\qquad=192\end{array}$ & $\begin{array}{c}\text { Interrupted } \\
N=588\end{array}$ & $\begin{array}{l}\text { Switch } \\
N=132\end{array}$ & $\begin{array}{c}\text { Polytherapy } \\
N=124\end{array}$ \\
\hline \multicolumn{5}{|c|}{ Total Healthcare Cost } \\
\hline \multicolumn{5}{|c|}{ Mean ( $( \pm s t d)$} \\
\hline Pre-index & $\begin{array}{c}\$ 13,157^{\mathrm{I}} \\
( \pm \$ 27,855)\end{array}$ & $\begin{array}{c}\$ 10,521^{\mathrm{C}} \\
( \pm \$ 16,613)\end{array}$ & $\begin{array}{c}\$ 13,336 \\
( \pm \$ 21,517)\end{array}$ & $\begin{array}{c}\$ 12,267 \\
(+\$ 13,244)\end{array}$ \\
\hline Post-index & $\begin{array}{l}\$ 19,960 \mathrm{SP} \\
( \pm \$ 32,312)\end{array}$ & $\begin{array}{c}\$ 20,486 \\
( \pm \$ 21,513)\end{array}$ & $\begin{array}{c}\$ 21,595^{\mathrm{C}} \\
( \pm \$ 24,226)\end{array}$ & $\begin{array}{c}\$ 27,741^{\mathrm{C}} \\
( \pm \$ 35,334)\end{array}$ \\
\hline \multicolumn{5}{|c|}{ Mental Healthcare Cost } \\
\hline \multicolumn{5}{|c|}{ Mean $(\underline{\text { std }})$} \\
\hline Pre-index & $\begin{array}{c}\$ 8,513^{\mathrm{I}} \\
( \pm \$ 24,121)\end{array}$ & $\begin{array}{c}\$ 6,168^{\mathrm{C}} \\
( \pm \$ 9,761)\end{array}$ & $\begin{array}{c}\$ 8,764 \\
( \pm \$ 11,778)\end{array}$ & $\begin{array}{c}\$ 8,641 \\
(+\$ 11,583)\end{array}$ \\
\hline Post-index & $\begin{array}{l}\$ 12,482^{\mathrm{ISP}} \\
( \pm \$ 24,195)\end{array}$ & $\begin{array}{c}\$ 17,890^{\mathrm{C}} \\
( \pm \$ 18,330)\end{array}$ & $\begin{array}{c}\$ 15,049^{C} \\
( \pm \$ 16,957)\end{array}$ & $\begin{array}{c}\$ 21,006^{\mathrm{C}} \\
( \pm \$ 35,334)\end{array}$ \\
\hline
\end{tabular}

\footnotetext{
${ }^{\text {I }}$ Significant difference between continuous (c) and interrupted cohorts at $\mathrm{p}<0.05$

${ }^{\mathrm{S}}$ Significant difference between continuous (c) and switch cohorts at $\mathrm{p}<0.05$

${ }^{\mathrm{P}}$ Significant difference between continuous (c) and polytherapy cohorts at $\mathrm{p}<0.05$
} 
Table 11a: Ordinary Least Squares (OLS) regression model for the impact pattern of antipsychotic use on total healthcare cost among schizophrenia patients (second stage sample selection models): Interrupted vs. Adherent therapy

\begin{tabular}{llll}
\hline Beta & S.E & t-statistic & Significance (p)
\end{tabular}

Demographic characteristics

Whites (ref: non-whites)

Metro (ref: non-metro)
Age (in years)

Males (ref: females)

0.005

0.571

0.120

$-0.152$

0.798

0.000

0.719

0.472

Schizophrenia subtype (ref: only schizophrenia)

Schizoaffective disorder

$$
0.069
$$

0.096

0.709

0.478

Both schizoaffective disorder and schizophrenia

$-0.124$

0.113

$-1.095$

0.274

Pre-index co-morbidities

Charlson Comorbidity Index

Alcohol and substance abuse

0.215

0.030

7.208

0.000

Bipolar disorder

$$
0.294
$$

0.107

0.103

0.128

2.731

0.006

Major depression

0.055

0.112

0.015

0.088

0.804

0.422

Other mental comorbidities

\section{Pre-index healthcare utilization and cost}

Pre-index total health related cost

$$
0.129
$$

0.023

5.683

0.000

Pre-index total health related

ER visits

0.081

0.092

0.880

0.379

Pre-index total health related hospitalizations
0.187

0.112

1.667

0.096

Pre-index total health related physician visits
0.005
1.006

0.315 
Table 11a: Ordinary Least Squares (OLS) regression model: Interrupted vs. Adherent therapy (contd.)

Beta $\quad S . E \quad t$-statistic $\quad$ Significance (p)

Year of index prescription (ref: 1999)
- 2000
$-0.010$
0.117
$-0.088$
0.930
- 2001
$-0.438$
0.120
$-3.656$
0.000

Pre-index period psychiatric medication use ( days of supply)

$\begin{array}{lcccc}\text { Mood stabilizers } & 0.021 & 0.010 & 1.966 & 0.049 \\ \text { Antipsychotics } & -0.002 & 0.001 & -1.710 & 0.087 \\ \text { Antidepressants } & 0.282 & 0.083 & 3.404 & 0.001 \\ \text { Benzodiazepines } & 0.082 & 0.082 & 1.002 & 0.317 \\ \text { Index prescription (ref: quetiapine) } & & & \\ \text { Risperidone } & -0.865 & 0.904 & -0.958 & 0.338 \\ \text { Olanzapine } & -0.666 & 0.000 & -0.606 & 0.544 \\ \text { Typicals } & 0.020 & 0.852 & -1.556 & 0.120 \\ \text { Interrupted (ref: Adherent) } & 0.078 & 0.466 & 2.314 & 0.021 \\ \text { Inverse Mills Ratio } & 0.403 & 0.280 & 1.441 & 0.150\end{array}$

\section{Model fit statistics:}

Adjusted R-square $=29.58 \%, \boldsymbol{F}=13.59, \boldsymbol{p}=0.00$ 
Table 11b: Ordinary Least Squares (OLS) regression model for the impact pattern of antipsychotic use on total healthcare cost among schizophrenia patients (second stage sample selection models): Switching vs. adherent therapy

\begin{tabular}{|c|c|c|c|c|}
\hline & Beta & S.E & t-statistic & Significance (p) \\
\hline \multicolumn{5}{|l|}{ Demographic characteristics } \\
\hline Age (in years) & 0.012 & 0.004 & 2.663 & 0.008 \\
\hline Males (ref: females) & -0.834 & 0.001 & -0.153 & 0.879 \\
\hline Whites (ref: non-whites) & 0.338 & 0.190 & 1.783 & 0.076 \\
\hline Metro (ref: non-metro) & -0.036 & 0.136 & -0.265 & 0.791 \\
\hline \multicolumn{5}{|l|}{ Prescribing physician type } \\
\hline Psychiatric prescriber & 0.277 & 0.000 & 0.202 & 0.840 \\
\hline \multicolumn{5}{|c|}{ Schizophrenia subtype (ref: only schizophrenia) } \\
\hline Schizoaffective disorder & -0.001 & 0.134 & -0.006 & 0.995 \\
\hline $\begin{array}{l}\text { Both schizoaffective disorder } \\
\text { and schizophrenia }\end{array}$ & -0.108 & 0.149 & -0.721 & 0.471 \\
\hline Charlson Comorbidity Index & 0.130 & 0.039 & 3.320 & 0.001 \\
\hline \multicolumn{5}{|l|}{ Pre-index co-morbidities } \\
\hline Alcohol and substance abuse & 0.254 & 0.137 & 1.855 & 0.065 \\
\hline Bipolar disorder & 0.088 & 0.166 & 0.530 & 0.597 \\
\hline Major depression & -0.169 & 0.136 & -1.238 & 0.217 \\
\hline Other mental comorbidities & 0.152 & 0.120 & 1.270 & 0.205 \\
\hline \multicolumn{5}{|c|}{ Pre-index healthcare utilization and cost } \\
\hline $\begin{array}{l}\text { Pre-index total health related } \\
\text { cost }\end{array}$ & 0.128 & 0.034 & 3.735 & 0.000 \\
\hline $\begin{array}{l}\text { Pre-index total health related } \\
\text { ER visits }\end{array}$ & -0.121 & 0.125 & -0.962 & 0.337 \\
\hline $\begin{array}{l}\text { Pre-index total health related } \\
\text { hospitalizations }\end{array}$ & -0.105 & 0.155 & -0.679 & 0.498 \\
\hline $\begin{array}{l}\text { Pre-index total health related } \\
\text { physician visits }\end{array}$ & 0.002 & 0.006 & 0.292 & 0.770 \\
\hline
\end{tabular}


Table 11b: Ordinary Least Squares (OLS) regression model: Switching vs. adherent therapy (contd.)

Beta $\quad$ S.E $\quad$ t-statistic $\quad$ Significance (p)

Year of index prescription (ref: 1999)
- 2000
0.209
0.149
1.399
0.163
- 2001
$-0.124$
0.142
$-0.874$
0.383

Pre-index period psychiatric medication use ( days of supply)

Mood stabilizers

0.021

0.000

2.971

0.003

Antipsychotics

$-0.001$

0.001

$-0.421$

0.674

Antidepressants

0.039

0.116

0.335

0.738

Benzodiazepines

0.110

0.108

1.012

0.312

Index prescription (ref: quetiapine)

\begin{tabular}{lllll} 
Risperidone & 0.001 & 0.000 & 1.149 & 0.252 \\
Olanzapine & 0.001 & 0.000 & 0.839 & 0.402 \\
Typicals & -0.020 & 0.000 & -2.429 & 0.016 \\
Switch (ref: Adherent) & 0.559 & 0.331 & 1.688 & 0.092 \\
Inverse Mills Ratio & -0.262 & 0.209 & -1.253 & 0.211 \\
\hline
\end{tabular}

\section{Model fit statistics:}

Adjusted $\boldsymbol{R}$-square $=23.4 \%, \boldsymbol{F}=4.84, \mathrm{p}=0.00$ 
Table 11c: Ordinary Least Squares (OLS) regression model for the impact pattern of antipsychotic use on total healthcare cost among schizophrenia patients (second stage sample selection models): Polytherapy vs. adherent therapy

\begin{tabular}{|c|c|c|c|c|}
\hline & Beta & S.E & t-statistic & Significance (p) \\
\hline \multicolumn{5}{|l|}{ Demographic characteristics } \\
\hline Age (in years) & 0.010 & 0.005 & 2.007 & 0.046 \\
\hline Males (ref: females) & -0.002 & 0.000 & -4.055 & 0.000 \\
\hline Whites (ref: non-whites) & 0.502 & 0.181 & 2.778 & 0.006 \\
\hline Metro (ref: non-metro) & 0.002 & 0.136 & 0.017 & 0.987 \\
\hline \multicolumn{5}{|l|}{ Prescribing physician type } \\
\hline Psychiatric prescriber & 0.000 & 0.000 & -1.406 & 0.161 \\
\hline \multicolumn{5}{|c|}{ Schizophrenia subtype (ref: only schizophrenia) } \\
\hline Schizoaffective disorder & 0.049 & 0.148 & 0.330 & 0.742 \\
\hline $\begin{array}{l}\text { Both schizoaffective disorder } \\
\text { and schizophrenia }\end{array}$ & -0.458 & 0.171 & -2.687 & 0.008 \\
\hline Charlson Comorbidity Index & 0.117 & 0.047 & 2.513 & 0.013 \\
\hline \multicolumn{5}{|l|}{ Pre-index co-morbidities } \\
\hline Alcohol and substance abuse & 0.440 & 0.155 & 2.842 & 0.005 \\
\hline Bipolar disorder & 0.272 & 0.195 & 1.396 & 0.164 \\
\hline Major depression & 0.133 & 0.159 & 0.838 & 0.403 \\
\hline Other mental comorbidities & 0.171 & 0.140 & 1.222 & 0.223 \\
\hline \multicolumn{5}{|c|}{ Pre-index healthcare utilization and cost } \\
\hline $\begin{array}{l}\text { Pre-index total health related } \\
\text { cost }\end{array}$ & 0.107 & 0.022 & 4.861 & 0.000 \\
\hline $\begin{array}{l}\text { Pre-index total health related } \\
\text { ER visits }\end{array}$ & -0.125 & 0.120 & -1.040 & 0.299 \\
\hline $\begin{array}{l}\text { Pre-index total health related } \\
\text { hospitalizations }\end{array}$ & -0.041 & 0.180 & -0.228 & 0.820 \\
\hline $\begin{array}{l}\text { Pre-index total health related } \\
\text { physician visits }\end{array}$ & 0.012 & 0.009 & 1.293 & 0.197 \\
\hline
\end{tabular}


Table 11c: Ordinary Least Squares (OLS) regression model: Polytherapy vs. adherent therapy (contd.)

\begin{tabular}{|c|c|c|}
\hline Beta & S.E & t-statistic \\
\hline
\end{tabular}

Year of index prescription (ref: 1999)
- 2000
0.000
0.157
0.001
0.999
- 2001
$-0.281$
0.162
$-1.734$
0.084

Pre-index period psychiatric medication use ( days of supply)

$\begin{array}{lcccc}\text { Mood stabilizers } & 0.211 & 0.000 & 3.487 & 0.001 \\ \text { Antipsychotics } & -0.001 & 0.001 & -1.457 & 0.146 \\ \text { Antidepressants } & 0.073 & 0.127 & 0.572 & 0.568 \\ \text { Benzodiazepines } & 0.075 & 0.120 & 0.627 & 0.531 \\ \text { Index prescription (ref: quetiapine) } & & & \\ \text { Risperidone } & 0.133 & 0.000 & 1.338 & 0.182 \\ \text { Olanzapine } & 0.078 & 0.000 & 0.989 & 0.324 \\ \text { Typicals } & 0.026 & 0.000 & 2.935 & 0.004 \\ \text { Polytherapy (ref: Adherent) } & 0.252 & 0.483 & 0.523 & 0.015 \\ \text { Inverse Mills Ratio } & 0.466 & 0.285 & 1.633 & 0.104\end{array}$

\section{Model fit statistics:}

Adjusted R-square $=28.6 \%, F=5.87, \boldsymbol{p}=0.00$ 
Table 12a: Ordinary Least Squares (OLS) regression model for the impact pattern of antipsychotic use on mental healthcare cost among schizophrenia patients (second stage sample selection models): Interrupted vs. adherent therapy

\begin{tabular}{|c|c|c|c|c|}
\hline & Beta & S.E & t-statistic & Significance (p) \\
\hline \multicolumn{5}{|l|}{ Demographic characteristics } \\
\hline Age (in years) & 0.002 & 0.004 & 0.498 & 0.619 \\
\hline Males (ref: females) & 0.002 & 0.000 & 3.337 & 0.001 \\
\hline Whites (ref: non-whites) & 0.092 & 0.121 & 0.762 & 0.446 \\
\hline Metro (ref: non-metro) & -0.141 & 0.100 & -1.400 & 0.162 \\
\hline \multicolumn{5}{|l|}{ Prescribing physician type } \\
\hline Psychiatric prescriber & 0.000 & 0.000 & 1.655 & 0.098 \\
\hline \multicolumn{5}{|c|}{ Schizophrenia subtype (ref: only schizophrenia) } \\
\hline Schizoaffective disorder & 0.094 & 0.106 & 0.889 & 0.374 \\
\hline $\begin{array}{l}\text { Both schizoaffective disorder } \\
\text { and schizophrenia }\end{array}$ & -0.423 & 0.122 & -3.464 & 0.001 \\
\hline Charlson Comorbidity Index & 0.053 & 0.045 & 1.184 & 0.236 \\
\hline \multicolumn{5}{|l|}{ Pre-index co-morbidities } \\
\hline Alcohol and substance abuse & 0.369 & 0.132 & 2.808 & 0.005 \\
\hline Bipolar disorder & 0.112 & 0.149 & 0.754 & 0.451 \\
\hline Major depression & 0.118 & 0.122 & 0.962 & 0.336 \\
\hline Other mental comorbidities & 0.013 & 0.099 & 0.132 & 0.895 \\
\hline \multicolumn{5}{|c|}{ Pre-index healthcare utilization and cost } \\
\hline $\begin{array}{l}\text { Pre-index mental-health } \\
\text { related cost }\end{array}$ & 0.192 & 0.093 & 2.069 & 0.039 \\
\hline $\begin{array}{l}\text { Pre-index mental-health } \\
\text { related ER visits }\end{array}$ & -0.032 & 0.129 & -0.244 & 0.807 \\
\hline $\begin{array}{l}\text { Pre-index mental-health } \\
\text { related hospitalizations }\end{array}$ & 0.306 & 0.103 & 2.960 & 0.003 \\
\hline $\begin{array}{l}\text { Pre-index mental-health } \\
\text { related physician visits }\end{array}$ & 0.379 & 0.139 & 2.726 & 0.006 \\
\hline
\end{tabular}


Table 12a: Ordinary Least Squares (OLS) regression model: Interrupted vs. adherent therapy (contd.)

Beta S.E t-statistic Significance (p)

Year of index prescription (ref: 1999)
- 2000
$-0.021$
0.126
$-0.163$
0.870
- 2001
$-0.334$
0.133
$-2.520$
0.012

Pre-index period psychiatric medication use ( days of supply)

$\begin{array}{lcccc}\text { Mood stabilizers } & 0.051 & 0.000 & 2.472 & 0.014 \\ \text { Antipsychotics } & 0.001 & 0.002 & 0.450 & 0.653 \\ \text { Antidepressants } & 0.419 & 0.227 & 1.848 & 0.065 \\ \text { Benzodiazepines } & -0.582 & 0.529 & -1.100 & 0.271 \\ \text { Index prescription (ref: quetiapine) } & & & \\ \text { Risperidone } & 0.735 & 0.000 & 0.709 & 0.478 \\ \text { Olanzapine } & 0.024 & 0.000 & 2.985 & 0.303 \\ \text { Typicals } & -0.033 & 0.995 & -3.344 & 0.001 \\ \text { Interrupted (ref: Adherent) } & 0.034 & 0.015 & 2.318 & 0.020 \\ \text { Inverse Mills Ratio } & 0.038 & 0.322 & 0.118 & 0.906\end{array}$

\section{Model fit statistics:}

Adjusted $R$-square $=26.79 \%, F=11.26, \boldsymbol{p}=0.00$ 
Table 12b: Ordinary Least Squares (OLS) regression model for the impact pattern of antipsychotic use on mental healthcare cost among schizophrenia patients (second stage sample selection models): Switch vs. adherent therapy

\begin{tabular}{|c|c|c|c|c|}
\hline & Beta & S.E & t-statistic & Significance (p) \\
\hline \multicolumn{5}{|l|}{ Demographic characteristics } \\
\hline Age (in years) & 0.014 & 0.004 & 3.167 & 0.002 \\
\hline Males (ref: females) & 0.001 & 0.000 & 1.591 & 0.113 \\
\hline Whites (ref: non-whites) & 0.373 & 0.217 & 1.719 & 0.087 \\
\hline Metro (ref: non-metro) & 0.008 & 0.140 & 0.056 & 0.955 \\
\hline \multicolumn{5}{|l|}{ Prescribing physician type } \\
\hline Psychiatric prescriber & 0.000 & 0.000 & -1.075 & 0.283 \\
\hline \multicolumn{5}{|c|}{ Schizophrenia subtype (ref: only schizophrenia) } \\
\hline Schizoaffective disorder & 0.169 & 0.147 & 1.149 & 0.252 \\
\hline $\begin{array}{l}\text { Both schizoaffective disorder } \\
\text { and schizophrenia }\end{array}$ & -0.166 & 0.136 & -1.218 & 0.224 \\
\hline Charlson Comorbidity Index & -0.019 & 0.049 & -0.391 & 0.696 \\
\hline \multicolumn{5}{|l|}{ Pre-index co-morbidities } \\
\hline Alcohol and substance abuse & 0.274 & 0.151 & 1.810 & 0.071 \\
\hline Bipolar disorder & 0.130 & 0.161 & 0.809 & 0.419 \\
\hline Major depression & -0.237 & 0.144 & -1.648 & 0.100 \\
\hline Other mental comorbidities & 0.033 & 0.125 & 0.263 & 0.793 \\
\hline \multicolumn{5}{|c|}{ Pre-index healthcare utilization and cost } \\
\hline $\begin{array}{l}\text { Pre-index mental-health } \\
\text { related cost }\end{array}$ & 0.042 & 0.017 & 2.495 & 0.013 \\
\hline $\begin{array}{l}\text { Pre-index mental-health } \\
\text { related ER visits }\end{array}$ & -0.243 & 0.153 & -1.592 & 0.113 \\
\hline $\begin{array}{l}\text { Pre-index mental-health } \\
\text { related hospitalizations }\end{array}$ & 0.054 & 0.124 & 0.431 & 0.667 \\
\hline $\begin{array}{l}\text { Pre-index mental-health } \\
\text { related physician visits }\end{array}$ & 0.433 & 0.065 & 6.680 & 0.000 \\
\hline
\end{tabular}


Table 12b: Ordinary Least Squares (OLS) regression model: Switch vs. adherent therapy (contd.)

Beta S.E t-statistic Significance (p)

Year of index prescription (ref: 1999)
- 2000
0.204
0.154
1.327
0.186
- 2001
$-0.122$
0.131
$-0.925$
0.356

Pre-index period psychiatric medication use ( days of supply)

$\begin{array}{lcccc}\text { Mood stabilizers } & 0.001 & 0.000 & 2.532 & 0.012 \\ \text { Antipsychotics } & -0.001 & 0.002 & -0.557 & 0.578 \\ \text { Antidepressants } & 0.357 & 0.296 & 1.205 & 0.229 \\ \text { Benzodiazepines } & -0.014 & 0.109 & -0.130 & 0.896 \\ \text { Index prescription (ref: quetiapine) } & & & \\ \text { Risperidone } & 0.020 & 0.000 & 1.382 & 0.168 \\ \text { Olanzapine } & 0.054 & 0.000 & 1.440 & 0.151 \\ \text { Typicals } & -0.020 & 0.000 & -3.105 & 0.002 \\ \text { Switch (ref: Adherent) } & 0.432 & 0.364 & 1.188 & 0.236 \\ \text { Inverse Mills Ratio } & -0.181 & 0.225 & -0.804 & 0.422\end{array}$

\section{Model fit statistics:}

Adjusted R-square $=23.3 \%, \mathrm{~F}=4.65, \mathrm{p}=0.00$ 
Table 12c: Ordinary Least Squares (OLS) regression model for the impact pattern of antipsychotic use on mental healthcare cost among schizophrenia patients (second stage sample selection models): Polytherapy vs. adherent therapy

\begin{tabular}{|c|c|c|c|c|}
\hline & Beta & S.E & t-statistic & Significance (p) \\
\hline \multicolumn{5}{|l|}{ Demographic characteristics } \\
\hline Age (in years) & 0.012 & 0.005 & 2.495 & 0.013 \\
\hline Males (ref: females) & -0.001 & 0.001 & -0.592 & 0.554 \\
\hline Whites (ref: non-whites) & 0.361 & 0.203 & 1.775 & 0.070 \\
\hline Metro (ref: non-metro) & -0.029 & 0.133 & -0.216 & 0.829 \\
\hline \multicolumn{5}{|l|}{ Prescribing physician type } \\
\hline Psychiatric prescriber & 0.000 & 0.000 & -1.498 & 0.135 \\
\hline \multicolumn{5}{|c|}{ Schizophrenia subtype (ref: only schizophrenia) } \\
\hline Schizoaffective disorder & 0.120 & 0.146 & 0.826 & 0.409 \\
\hline $\begin{array}{l}\text { Both schizoaffective disorder } \\
\text { and schizophrenia }\end{array}$ & -0.554 & 0.164 & -3.369 & 0.000 \\
\hline Charlson Comorbidity Index & -0.020 & 0.057 & -0.357 & 0.721 \\
\hline \multicolumn{5}{|l|}{ Pre-index co-morbidities } \\
\hline Alcohol and substance abuse & 0.471 & 0.166 & 2.830 & 0.005 \\
\hline Bipolar disorder & 0.381 & 0.199 & 1.916 & 0.056 \\
\hline Major depression & 0.028 & 0.172 & 0.162 & 0.871 \\
\hline Other mental comorbidities & 0.151 & 0.144 & 1.047 & 0.295 \\
\hline \multicolumn{5}{|c|}{ Pre-index healthcare utilization and cost } \\
\hline $\begin{array}{l}\text { Pre-index mental-health } \\
\text { related cost }\end{array}$ & 0.058 & 0.017 & 3.339 & 0.001 \\
\hline $\begin{array}{l}\text { Pre-index mental-health } \\
\text { related ER visits }\end{array}$ & 0.058 & 0.152 & 0.381 & 0.703 \\
\hline $\begin{array}{l}\text { Pre-index mental-health } \\
\text { related hospitalizations }\end{array}$ & -0.137 & 0.129 & -1.059 & 0.290 \\
\hline $\begin{array}{l}\text { Pre-index mental-health } \\
\text { related physician visits }\end{array}$ & 0.058 & 0.518 & 0.112 & 0.911 \\
\hline
\end{tabular}


Table 12c: Ordinary Least Squares (OLS) regression model: Polytherapy vs. adherent therapy (contd.)

Beta S.E t-statistic Significance (p)

Year of index prescription (ref: 1999)
- 2000
0.114
0.164
0.695
0.487
- 2001
$-0.180$
0.149
$-1.211$
0.220

Pre-index period psychiatric medication use ( days of supply)

Mood stabilizers

0.021

0.000

1.787

0.070

Antipsychotics

$-0.001$

0.001

$-1.173$

0.241

Antidepressants

0.104

0.132

0.787

0.431

Benzodiazepines

$-0.071$

0.123

$-0.572$

0.567

Index prescription (ref: quetiapine)

Risperidone

0.030

0.000

1.452

0.147

Olanzapine

0.034

0.000

1.683

0.093

Typicals

$-0.095$

0.000

$-3.013$

0.002

Polytherapy (ref: Adherent)

0.128

0.045

2.833

0.005

Inverse Mills Ratio

0.349

0.306

1.139

0.256

\section{Model fit statistics:}

Adjusted R-square $=28.8 \%, \boldsymbol{F}=5.57, \boldsymbol{p}=0.00$ 
Table 13: Total and mental healthcare costs associated with the different antipsychotic utilization patterns among bipolar disorder patients

\begin{tabular}{|c|c|c|c|c|}
\hline & $\begin{array}{c}\text { Continuous } \\
N=166\end{array}$ & $\begin{array}{c}\text { Interrupted } \\
N=484\end{array}$ & $\begin{array}{l}\text { Switch } \\
N=111\end{array}$ & $\begin{array}{c}\text { Polytherapy } \\
N=64\end{array}$ \\
\hline \multicolumn{5}{|c|}{ Total Healthcare Cost } \\
\hline \multicolumn{5}{|c|}{ Mean $( \pm s t d)$} \\
\hline Pre-index & $\begin{array}{c}\$ 12,220 \\
(+\$ 26,609)\end{array}$ & $\begin{array}{c}\$ 10,521 \\
( \pm \$ 16,613)\end{array}$ & $\begin{array}{c}\$ 12,630 \\
( \pm \$ 20,699)\end{array}$ & $\begin{array}{c}\$ 10,664 \\
( \pm \$ 9,695)\end{array}$ \\
\hline Post-index & $\begin{array}{c}\$ 18,383^{\mathrm{P}} \\
( \pm \$ 30,283)\end{array}$ & $\begin{array}{c}\$ 20,486^{\mathrm{C}} \\
( \pm \$ 21,513)\end{array}$ & $\begin{array}{c}\$ 20,104^{\mathrm{C}} \\
( \pm \$ 23,116)\end{array}$ & $\begin{array}{l}\$ 26,972^{C} \\
( \pm \$ 32,885)\end{array}$ \\
\hline \multicolumn{5}{|c|}{ Mental Healthcare Cost } \\
\hline \multicolumn{5}{|c|}{ Mean ( } \\
\hline Pre-index & $\begin{array}{c}\$ 7,389^{\mathrm{I}} \\
( \pm 21,824)\end{array}$ & $\begin{array}{l}\$ 6,168^{\mathrm{C}} \\
( \pm \$ 9,761)\end{array}$ & $\begin{array}{c}\$ 7,652 \\
( \pm \$ 10,565)\end{array}$ & $\begin{array}{c}\$ 6,243 \\
( \pm \$ 7,247)\end{array}$ \\
\hline Post-index & $\begin{array}{l}\$ 11,134^{\text {I P }} \\
( \pm \$ 21,901)\end{array}$ & $\begin{array}{c}\$ 17,890^{\mathrm{C}} \\
( \pm \$ 18,330)\end{array}$ & $\begin{array}{c}\$ 12,758 \\
(+\$ 15,133)\end{array}$ & $\begin{array}{c}\$ 16,470^{C} \\
( \pm \$ 15,258)\end{array}$ \\
\hline
\end{tabular}

${ }^{\text {I }}$ Significant difference between continuous (c) and interrupted cohorts at $\mathrm{p}<0.05$

${ }^{\mathrm{S}}$ Significant difference between continuous (c) and switch cohorts at $\mathrm{p}<0.05$

${ }^{\mathrm{P}}$ Significant difference between continuous (c) and polytherapy cohorts at $\mathrm{p}<0.05$ 
Table 14a: Ordinary Least Squares (OLS) regression model for the impact pattern of antipsychotic use on total healthcare cost among bipolar disorder patients (second stage sample selection models): Interrupted vs. adherent therapy

\begin{tabular}{|c|c|c|c|c|}
\hline & Beta & S.E & t-statistic & Significance (p) \\
\hline Age (in years) & 0.006 & 0.004 & 1.575 & 0.115 \\
\hline $\begin{array}{l}18 \text { years or above (ref: less } \\
\text { than } 18 \text { years) }\end{array}$ & 0.398 & 0.140 & 1.135 & 0.344 \\
\hline Males (ref: females) & 0.512 & 0.000 & 0.015 & 0.988 \\
\hline Whites (ref: non-whites) & 0.253 & 0.121 & 2.090 & 0.037 \\
\hline Metro (ref: non-metro) & -0.134 & 0.104 & -1.278 & 0.201 \\
\hline Psychiatric prescriber & 0.000 & 0.000 & 1.014 & 0.311 \\
\hline \multicolumn{5}{|c|}{ Bipolar Subtype (ref: bipolar disorder I) } \\
\hline Bipolar disorder II & 0.130 & 0.111 & 1.165 & 0.244 \\
\hline Mixed & -0.084 & 0.127 & -0.657 & 0.511 \\
\hline $\begin{array}{l}\text { Pre-index co-morbidities } \\
\text { Charlson Comorbidity Index }\end{array}$ & 0.213 & 0.032 & 6.744 & 0.000 \\
\hline Alcohol and substance abuse & 0.309 & 0.126 & 2.449 & 0.014 \\
\hline Bipolar disorder & 0.010 & 0.140 & 0.072 & 0.943 \\
\hline Major depression & 0.113 & 0.123 & 0.919 & 0.358 \\
\hline Other mental comorbidities & 0.056 & 0.096 & 0.584 & 0.559 \\
\hline $\begin{array}{l}\text { Pre-index mental-health } \\
\text { related cost }\end{array}$ & 0.137 & 0.024 & 5.778 & 0.000 \\
\hline $\begin{array}{l}\text { Pre-index mental-health } \\
\text { related ER visits }\end{array}$ & 0.042 & 0.099 & 0.428 & 0.669 \\
\hline $\begin{array}{l}\text { Pre-index mental-health } \\
\text { related hospitalizations }\end{array}$ & 0.158 & 0.122 & 1.294 & 0.196 \\
\hline $\begin{array}{l}\text { Pre-index mental-health } \\
\text { related physician visits }\end{array}$ & 0.007 & 0.006 & 1.289 & 0.197 \\
\hline \multicolumn{5}{|c|}{ Year of index prescription (ref: 1999) } \\
\hline - 2000 & -0.001 & 0.126 & -0.010 & 0.992 \\
\hline - 2001 & -0.481 & 0.129 & -3.712 & 0.000 \\
\hline
\end{tabular}


Table 14a: Ordinary Least Squares (OLS) regression model: Interrupted vs. adherent therapy (contd.)

\begin{tabular}{lcccc}
\hline & Beta & S.E & t-statistic & Significance (p) \\
\hline Pre-index period psychiatric medication & use & ( days of supply) & \\
Mood stabilizers & 0.001 & 0.000 & 1.613 & 0.107 \\
Antipsychotics & -0.002 & 0.002 & -1.282 & 0.200 \\
Antidepressants & 0.262 & 0.090 & 2.927 & 0.003 \\
Benzodiazepines & 0.052 & 0.090 & 0.583 & 0.560 \\
Index prescription (ref: quetiapine) & & & & \\
Risperidone & -0.951 & 0.000 & -0.919 & 0.358 \\
Olanzapine & -0.997 & 0.000 & -0.794 & 0.427 \\
Typicals & 0.000 & 0.917 & -1.687 & 0.092 \\
Interrupted (ref: Adherent) & 0.016 & 0.451 & 2.126 & 0.034 \\
Inverse Mills Ratio & -0.295 & 0.271 & -1.088 & 0.276 \\
\hline
\end{tabular}

\section{Model fit statistics:}

Adjusted R-square $=30.20 \%, \mathrm{~F}=23.45, \mathrm{p}=0.000$ 
Table 14b: Ordinary Least Squares (OLS) regression model for the impact pattern of antipsychotic use on total healthcare cost among bipolar disorder patients (second stage sample selection models): Switching vs. adherent therapy

\begin{tabular}{|c|c|c|c|c|}
\hline & Beta & S.E & t-statistic & Significance (p) \\
\hline \multicolumn{5}{|l|}{ Demographic characteristics } \\
\hline Age (in years) & 0.011 & 0.005 & 2.251 & 0.025 \\
\hline $\begin{array}{l}18 \text { years or above (ref: less } \\
\text { than } 18 \text { years) }\end{array}$ & 0.568 & 0.130 & 1.135 & 0.524 \\
\hline Males (ref: females) & 0.000 & 0.001 & -0.183 & 0.855 \\
\hline Whites (ref: non-whites) & 0.423 & 0.218 & 1.942 & 0.053 \\
\hline Metro (ref: non-metro) & -0.164 & 0.144 & -1.141 & 0.255 \\
\hline $\begin{array}{l}\text { Prescribing physician type } \\
\text { Psychiatric prescriber }\end{array}$ & -0.170 & 0.000 & -0.012 & 0.990 \\
\hline \multicolumn{5}{|c|}{ Bipolar Subtype (ref: bipolar disorder I) } \\
\hline Bipolar disorder II & -0.049 & 0.150 & -0.324 & 0.746 \\
\hline Mixed & -0.167 & 0.158 & -1.057 & 0.292 \\
\hline $\begin{array}{l}\text { Pre-index co-morbidities } \\
\text { Charlson Comorbidity Index }\end{array}$ & 0.125 & 0.041 & 3.070 & 0.002 \\
\hline Alcohol and substance abuse & 0.185 & 0.161 & 1.151 & 0.251 \\
\hline Bipolar disorder & 0.112 & 0.189 & 0.593 & 0.553 \\
\hline Major depression & -0.228 & 0.155 & -1.475 & 0.141 \\
\hline Other mental comorbidities & 0.173 & 0.128 & 1.353 & 0.177 \\
\hline $\begin{array}{l}\text { Pre-index mental-health } \\
\text { related cost }\end{array}$ & 0.123 & 0.031 & 3.963 & 0.000 \\
\hline $\begin{array}{l}\text { Pre-index mental-health } \\
\text { related ER visits }\end{array}$ & -0.105 & 0.129 & -0.811 & 0.418 \\
\hline $\begin{array}{l}\text { Pre-index mental-health } \\
\text { related hospitalizations }\end{array}$ & -0.178 & 0.156 & -1.144 & 0.254 \\
\hline $\begin{array}{l}\text { Pre-index mental-health } \\
\text { related physician visits }\end{array}$ & 0.007 & 0.007 & 0.958 & 0.339 \\
\hline \multicolumn{5}{|c|}{ Year of index prescription (ref: 1999) } \\
\hline - 2000 & 0.197 & 0.160 & 1.237 & 0.217 \\
\hline - 2001 & -0.150 & 0.153 & -0.978 & 0.329 \\
\hline
\end{tabular}


Table 14b: Ordinary Least Squares (OLS) regression model: Switching vs. adherent therapy (contd.)

\begin{tabular}{lcccc}
\hline & Beta & S.E & t-statistic & Significance (p) \\
\hline Pre-index period psychiatric medication & use & ( days of supply) & \\
Mood stabilizers & 0.001 & 0.000 & 2.477 & 0.014 \\
Antipsychotics & -0.001 & 0.001 & -0.819 & 0.414 \\
Antidepressants & 0.097 & 0.118 & 0.821 & 0.412 \\
Benzodiazepines & 0.016 & 0.116 & 0.140 & 0.889 \\
Index prescription (ref: quetiapine) & & & & \\
Risperidone & 0.631 & 0.000 & 0.532 & 0.596 \\
Olanzapine & 0.830 & 0.000 & 0.629 & 0.530 \\
Typicals & 0.030 & 0.000 & 2.451 & 0.015 \\
Switch (ref: Adherent) & 0.163 & 0.373 & 2.517 & 0.030 \\
Inverse Mills Ratio & -0.293 & 0.235 & -1.245 & 0.214 \\
\hline
\end{tabular}

\section{Model fit statistics:}

Adjusted R-square $=23.45 \%, \mathrm{~F}=4.13, \mathrm{p}=0.000$ 
Table 14c: Ordinary Least Squares (OLS) regression model for the impact pattern of antipsychotic use on total healthcare cost among bipolar disorder patients (second stage sample selection models): Polytherapy vs. adherent therapy

\begin{tabular}{|c|c|c|c|c|}
\hline & Beta & S.E & t-statistic & Significance (p) \\
\hline \multicolumn{5}{|l|}{ Demographic characteristics } \\
\hline Age (in years) & 0.011 & 0.006 & 1.905 & 0.058 \\
\hline $\begin{array}{l}18 \text { years or above (ref: less } \\
\text { than } 18 \text { years) }\end{array}$ & 0.244 & 0.135 & 1.156 & 0.444 \\
\hline Males (ref: females) & -0.001 & 0.001 & -1.999 & 0.047 \\
\hline Whites (ref: non-whites) & 0.604 & 0.223 & 2.712 & 0.007 \\
\hline Metro (ref: non-metro) & 0.062 & 0.162 & 0.380 & 0.705 \\
\hline $\begin{array}{l}\text { Prescribing physician type } \\
\text { Psychiatric prescriber }\end{array}$ & 0.020 & 0.010 & -1.193 & 0.234 \\
\hline \multicolumn{5}{|c|}{ Bipolar Subtype (ref: bipolar disorder I) } \\
\hline Bipolar disorder II & 0.087 & 0.167 & 0.519 & 0.605 \\
\hline Mixed & -0.344 & 0.192 & -1.798 & 0.074 \\
\hline $\begin{array}{l}\text { Pre-index co-morbidities } \\
\text { Charlson Comorbidity Index }\end{array}$ & 0.115 & 0.057 & 2.018 & 0.045 \\
\hline Alcohol and substance abuse & 0.376 & 0.166 & 2.263 & 0.025 \\
\hline Bipolar disorder & 0.154 & 0.190 & 0.810 & 0.419 \\
\hline Major depression & 0.066 & 0.172 & 0.383 & 0.702 \\
\hline Other mental comorbidities & 0.202 & 0.153 & 1.318 & 0.189 \\
\hline $\begin{array}{l}\text { Pre-index mental-health } \\
\text { related cost }\end{array}$ & 0.108 & 0.025 & 4.366 & 0.000 \\
\hline $\begin{array}{l}\text { Pre-index mental-health } \\
\text { related ER visits }\end{array}$ & -0.118 & 0.137 & -0.859 & 0.391 \\
\hline $\begin{array}{l}\text { Pre-index mental-health } \\
\text { related hospitalizations }\end{array}$ & -0.056 & 0.213 & -0.263 & 0.793 \\
\hline $\begin{array}{l}\text { Pre-index mental-health } \\
\text { related physician visits }\end{array}$ & 0.016 & 0.011 & 1.480 & 0.140 \\
\hline \multicolumn{5}{|c|}{ Year of index prescription (ref: 1999) } \\
\hline - 2000 & 0.027 & 0.180 & 0.152 & 0.879 \\
\hline
\end{tabular}


Table 14c: Ordinary Least Squares (OLS) regression model: Polytherapy vs. adherent therapy (contd.)

\begin{tabular}{lcccc}
\hline & Beta & S.E & t-statistic & Significance (p) \\
\hline \multicolumn{1}{c}{2001} & -0.345 & 0.184 & -1.876 & 0.062 \\
Pre-index period psychiatric medication & use & ( days & of supply) & \\
Mood stabilizers & 0.021 & 0.000 & 2.305 & 0.022 \\
Antipsychotics & -0.001 & 0.001 & -1.350 & 0.179 \\
Antidepressants & 0.111 & 0.148 & 0.752 & 0.453 \\
Benzodiazepines & -0.055 & 0.138 & -0.399 & 0.690 \\
Index prescription (ref: quetiapine) & & & \\
Risperidone & 0.002 & 0.020 & 1.402 & 0.162 \\
Olanzapine & 0.002 & 0.001 & 1.057 & 0.292 \\
Typicals & 0.036 & 0.010 & 2.452 & 0.021 \\
Polytherapy (ref: Adherent) & 0.320 & 0.504 & 2.154 & 0.007 \\
Inverse Mills Ratio & -0.298 & 0.309 & -0.963 & 0.337 \\
\hline
\end{tabular}

\section{Model fit statistics:}

Adjusted R-square $=25.7 \%, F=4.02, p=0.000$ 
Table 15a: Ordinary Least Squares (OLS) regression model for the impact pattern of antipsychotic use on mental healthcare cost among bipolar disorder patients (second stage sample selection models): Interrupted vs. adherent therapy

\begin{tabular}{|c|c|c|c|c|}
\hline & Beta & S.E & t-statistic & Significance (p) \\
\hline \multicolumn{5}{|l|}{ Demographic characteristics } \\
\hline Age (in years) & 0.002 & 0.004 & 0.418 & 0.676 \\
\hline $\begin{array}{l}18 \text { years or above (ref: less } \\
\text { than } 18 \text { years) }\end{array}$ & 0.543 & 0.342 & 1.360 & 0.561 \\
\hline Males (ref: females) & 0.002 & 0.001 & 3.898 & 0.000 \\
\hline Whites (ref: non-whites) & 0.163 & 0.139 & 1.174 & 0.241 \\
\hline Metro (ref: non-metro) & -0.073 & 0.115 & -0.638 & 0.524 \\
\hline Psychiatric prescriber & 0.000 & 0.000 & 2.007 & 0.045 \\
\hline \multicolumn{5}{|c|}{ Bipolar Subtype (ref: bipolar disorder I) } \\
\hline Bipolar disorder II & 0.047 & 0.124 & 0.378 & 0.706 \\
\hline Mixed & -0.408 & 0.136 & -2.999 & 0.003 \\
\hline Pre-index co-morbidities & 0066 & 0047 & 1391 & 0164 \\
\hline Alcohol and substance abuse & 0.381 & 0.156 & 2.441 & 0.015 \\
\hline Bipolar disorder & 0.059 & 0.158 & 0.371 & 0.711 \\
\hline Major depression & 0.198 & 0.129 & 1.527 & 0.127 \\
\hline Other mental comorbidities & 0.016 & 0.107 & 0.153 & 0.879 \\
\hline $\begin{array}{l}\text { Pre-index mental-health } \\
\text { related cost }\end{array}$ & 0.030 & 0.015 & 1.965 & 0.050 \\
\hline $\begin{array}{l}\text { Pre-index mental-health } \\
\text { related ER visits }\end{array}$ & -0.020 & 0.141 & -0.141 & 0.888 \\
\hline $\begin{array}{l}\text { Pre-index mental-health } \\
\text { related hospitalizations }\end{array}$ & 0.319 & 0.111 & 2.884 & 0.004 \\
\hline $\begin{array}{l}\text { Pre-index mental-health } \\
\text { related physician visits }\end{array}$ & 0.364 & 0.151 & 2.414 & 0.016 \\
\hline \multicolumn{5}{|c|}{ Year of index prescription (ref: 1999) } \\
\hline - 2000 & -0.059 & 0.134 & -0.438 & 0.661 \\
\hline - 2001 & -0.420 & 0.143 & -2.947 & 0.003 \\
\hline
\end{tabular}


Table 15a: Ordinary Least Squares (OLS) regression model: Interrupted vs. adherent therapy (contd.)

\begin{tabular}{lcccc}
\hline & Beta & S.E & t-statistic & Significance (p) \\
\hline Pre-index period psychiatric medication & use & ( days of supply) & \\
Mood stabilizers & 0.051 & 0.000 & 2.217 & 0.027 \\
Antipsychotics & 0.001 & 0.002 & 0.557 & 0.578 \\
Antidepressants & 0.245 & 0.250 & 0.981 & 0.327 \\
Benzodiazepines & 0.213 & 0.103 & 2.075 & 0.038 \\
Index prescription (ref: quetiapine) & & & & \\
Risperidone & 0.000 & 0.000 & 1.233 & 0.218 \\
Olanzapine & 0.001 & 0.000 & 1.139 & 0.072 \\
Typicals & 0.041 & 0.000 & 3.317 & 0.001 \\
Interrupted (ref: Adherent) & 0.069 & 0.503 & 2.330 & 0.015 \\
Inverse Mills Ratio & -0.103 & 0.305 & -0.336 & 0.737 \\
\hline
\end{tabular}

\section{Model fit statistics:}

Adjusted R-square $=28.18 \%, \mathrm{~F}=10.43, \mathrm{p}=0.000$ 
Table 15b: Ordinary Least Squares (OLS) regression model for the impact pattern of antipsychotic use on mental healthcare cost among bipolar disorder patients (second stage sample selection models): Switch vs. adherent therapy

\begin{tabular}{|c|c|c|c|c|}
\hline & Beta & S.E & t-statistic & Significance (p) \\
\hline \multicolumn{5}{|l|}{ Demographic characteristics } \\
\hline Age (in years) & 0.012 & 0.005 & 2.514 & 0.013 \\
\hline $\begin{array}{l}18 \text { years or above (ref: less } \\
\text { than } 18 \text { years) }\end{array}$ & 0.348 & 0.341 & 1.432 & 0.356 \\
\hline Males (ref: females) & 0.001 & 0.000 & 1.674 & 0.095 \\
\hline Whites (ref: non-whites) & 0.435 & 0.249 & 1.750 & 0.081 \\
\hline Metro (ref: non-metro) & -0.039 & 0.142 & -0.272 & 0.786 \\
\hline Psychiatric prescriber & 0.000 & 0.000 & -1.058 & 0.291 \\
\hline \multicolumn{5}{|c|}{ Bipolar Subtype (ref: bipolar disorder I) } \\
\hline Bipolar disorder II & 0.075 & 0.158 & 0.474 & 0.636 \\
\hline Mixed & -0.205 & 0.149 & -1.375 & 0.170 \\
\hline $\begin{array}{l}\text { Pre-index co-morbidities } \\
\text { Charlson Comorbidity Index }\end{array}$ & -0.018 & 0.049 & -0.358 & 0.720 \\
\hline Alcohol and substance abuse & 0.201 & 0.182 & 1.101 & 0.272 \\
\hline Bipolar disorder & 0.079 & 0.161 & 0.492 & 0.623 \\
\hline Major depression & -0.229 & 0.136 & -1.684 & 0.093 \\
\hline Other mental comorbidities & 0.009 & 0.128 & 0.070 & 0.944 \\
\hline $\begin{array}{l}\text { Pre-index mental-health } \\
\text { related cost }\end{array}$ & 0.041 & 0.016 & 2.502 & 0.013 \\
\hline $\begin{array}{l}\text { Pre-index mental-health } \\
\text { related ER visits }\end{array}$ & -0.259 & 0.160 & -1.617 & 0.107 \\
\hline $\begin{array}{l}\text { Pre-index mental-health } \\
\text { related hospitalizations }\end{array}$ & 0.068 & 0.127 & 0.535 & 0.593 \\
\hline $\begin{array}{l}\text { Pre-index mental-health } \\
\text { related physician visits }\end{array}$ & 0.519 & 0.070 & 7.457 & 0.000 \\
\hline \multicolumn{5}{|c|}{ Year of index prescription (ref: 1999) } \\
\hline - 2000 & 0.162 & 0.162 & 0.997 & 0.320 \\
\hline - 2001 & -0.209 & 0.130 & -1.603 & 0.110 \\
\hline
\end{tabular}


Table 15b: Ordinary Least Squares (OLS) regression model: Switch vs. adherent therapy (contd.)

\begin{tabular}{lcccc}
\hline & Beta & S.E & t-statistic & Significance (p) \\
\hline Pre-index period psychiatric medication & use & ( days of supply) & \\
Mood stabilizers & 0.001 & 0.000 & 2.253 & 0.025 \\
Antipsychotics & -0.002 & 0.002 & -0.889 & 0.375 \\
Antidepressants & 0.208 & 0.334 & 0.622 & 0.535 \\
Benzodiazepines & -0.090 & 0.116 & -0.769 & 0.443 \\
Index prescription (ref: quetiapine) & & & & \\
Risperidone & 0.040 & 0.000 & 1.172 & 0.242 \\
Olanzapine & 0.021 & 0.000 & 1.344 & 0.180 \\
Typicals & 0.040 & 0.000 & 2.753 & 0.006 \\
Switch (ref: Adherent) & 0.517 & 0.402 & 1.286 & 0.200 \\
Inverse Mills Ratio & -0.322 & 0.242 & -1.332 & 0.184 \\
\hline
\end{tabular}

\section{Model fit statistics:}

Adjusted R-square $=25.90 \%, \mathrm{~F}=4.57, \mathrm{p}=0.000$ 
Table 15c: Ordinary Least Squares (OLS) regression model for the impact pattern of antipsychotic use on mental healthcare cost among bipolar disorder patients (second stage sample selection models): Polytherapy vs. adherent therapy

\begin{tabular}{|c|c|c|c|c|}
\hline & Beta & S.E & t-statistic & Significance (p) \\
\hline \multicolumn{5}{|l|}{ Demographic characteristics } \\
\hline Age (in years) & 0.012 & 0.005 & 2.219 & 0.028 \\
\hline $\begin{array}{l}18 \text { years or above (ref: less } \\
\text { than } 18 \text { years) }\end{array}$ & 0.345 & 0.432 & 2.265 & 0.044 \\
\hline Males (ref: females) & 0.002 & 0.001 & 2.760 & 0.006 \\
\hline Whites (ref: non-whites) & 0.445 & 0.282 & 1.580 & 0.116 \\
\hline Metro (ref: non-metro) & 0.079 & 0.156 & 0.507 & 0.613 \\
\hline Psychiatric prescriber & 0.000 & 0.000 & -1.667 & 0.097 \\
\hline \multicolumn{5}{|c|}{ Bipolar Subtype (ref: bipolar disorder I) } \\
\hline Bipolar disorder II & 0.163 & 0.161 & 1.010 & 0.314 \\
\hline Mixed & -0.434 & 0.174 & -2.497 & 0.013 \\
\hline $\begin{array}{l}\text { Pre-index co-morbidities } \\
\text { Charlson Comorbidity Index }\end{array}$ & 0.038 & 0.051 & 0.739 & 0.461 \\
\hline Alcohol and substance abuse & 0.432 & 0.186 & 2.316 & 0.022 \\
\hline Bipolar disorder & 0.290 & 0.173 & 1.669 & 0.097 \\
\hline Major depression & -0.023 & 0.170 & -0.133 & 0.895 \\
\hline Other mental comorbidities & 0.137 & 0.157 & 0.872 & 0.384 \\
\hline $\begin{array}{l}\text { Pre-index mental-health } \\
\text { related cost }\end{array}$ & 0.044 & 0.016 & 2.686 & 0.008 \\
\hline $\begin{array}{l}\text { Pre-index mental-health } \\
\text { related ER visits }\end{array}$ & 0.110 & 0.166 & 0.661 & 0.510 \\
\hline $\begin{array}{l}\text { Pre-index mental-health } \\
\text { related hospitalizations }\end{array}$ & -0.073 & 0.138 & -0.528 & 0.598 \\
\hline $\begin{array}{l}\text { Pre-index mental-health } \\
\text { related physician visits }\end{array}$ & 0.116 & 0.043 & 2.689 & 0.008 \\
\hline \multicolumn{5}{|c|}{ Year of index prescription (ref: 1999) } \\
\hline - 2000 & 0.108 & 0.184 & 0.586 & 0.558 \\
\hline - 2001 & -0.285 & 0.154 & -1.848 & 0.066 \\
\hline
\end{tabular}


Table 15c: Ordinary Least Squares (OLS) regression model: Polytherapy vs. adherent therapy (contd.)

\begin{tabular}{lcccc}
\hline & Beta & S.E & t-statistic & Significance (p) \\
\hline Pre-index period psychiatric medication & use & ( days of supply) & \\
Mood stabilizers & 0.004 & 0.020 & 0.732 & 0.465 \\
Antipsychotics & -0.002 & 0.001 & -1.300 & 0.195 \\
Antidepressants & 0.059 & 0.157 & 0.377 & 0.707 \\
Benzodiazepines & -0.127 & 0.146 & -0.871 & 0.385 \\
Index prescription (ref: quetiapine) & & & & \\
Risperidone & 0.031 & 0.023 & 1.688 & 0.093 \\
Olanzapine & 0.050 & 0.017 & 2.029 & 0.084 \\
Typicals & 0.032 & 0.000 & 2.293 & 0.023 \\
Polytherapy (ref: Adherent) & 0.153 & 0.503 & 2.304 & 0.002 \\
Inverse Mills Ratio & -0.168 & 0.298 & -0.561 & 0.575 \\
\hline
\end{tabular}

\section{Model fit statistics:}

Adjusted $R$-square $=26.91 \%, \mathrm{~F}=4.22, \mathrm{p}=0.000$ 


\section{PHASE 2 - Results}

Schizophrenia patients initiated on multiple antipsychotics $(n=37)$ were excluded from the analysis in the phase 2. This resulted in sample size of 999 schizophrenia patients. Schizophrenia patients initiated on study antipsychotics were divided into the following study cohorts based on the first prescription of the index antipsychotic:

- Patients initiated on olanzapine: $346(34.63 \%)$

- Patients initiated on risperidone: $201(20.12 \%)$

- Patients initiated on quetiapine: $149(14.91 \%)$

- Patients initiated on typical antipsychotics: 303 (30.33\%)

There were 7 bipolar disorder patients who were initiated on multiple antipsychotics. After excluding these patients, the resulting sample size of bipolar disorder patients was 825 . Bipolar disorder patients initiated on study antipsychotics were divided into the following study cohorts based on the first prescription of the index antipsychotic:

- Patients initiated on olanzapine: $283(34.30 \%)$

- Patients initiated on risperidone: $231(28.00 \%)$

- Patients initiated on quetiapine: $106(12.85 \%)$

- Patients initiated on typical antipsychotics: 205 (24.85\%)

\section{Demographic characteristics of patients initiated on antipsychotics}

\section{$\underline{\text { Schizophrenia }}$}

Table 16 provides information on demographic characteristics and co-morbidities among schizophrenia patients initiated on antipsychotics. Wilcoxon rank sum test was 
used to compare continuous variables like age and Charlson comorbidity index. Chisquare analysis was used to compare the categorical variables.

Patients initiated on quetiapine did not differ significantly from patients initiated on other antipsychotics based on age, gender and race. Significantly higher proportion of patients initiated on quetiapine $(79.2 \%)$ were from non-metro areas compared to patients initiated on olanzapine (66.8\%) and typical antipsychotics (67.7\%). In addition, significantly higher proportion of patients initiated on quetiapine $(68.3 \%)$ had a psychiatric prescriber for the index prescription compared to patients initiated on olanzapine $(55.7 \%)$, risperidone $(55.3 \%)$, and typical antipsychotic $(42.5 \%)$.

Significantly higher proportion of patients initiated on quetiapine showed 'dual diagnosis' (claims associated with both diagnosis in the 12-months pre-index period) of schizophrenia and schizoaffective disorder (57.7\%) as compared to initiated olanzapine (39.6\%), risperidone $(37.3 \%)$ and typical antipsychotics $(31.3 \%)$. There were significantly a higher proportion of patients with alcohol and substance abuse in the quetiapine cohort (24.8\%) as compared to the risperidone (14.9\%) cohort. There was significantly greater proportion of patients with mild to moderate depression among the quetiapine cohort (13.4\%) as compared to the typical antipsychotic cohort (7.6\%).

\section{$\underline{\text { Bipolar Disorder }}$}

Table 17 provides information on demographic characteristics and co-morbidities among schizophrenia patients initiated on antipsychotics. Significantly greater proportions of patients initiated on quetiapine (90.6\%) were 18 years or older compared to patients initiated on risperidone $(63.2 \%)$. Significantly higher proportion of patients 
initiated on quetiapine ( $84.0 \%)$ were from non-metro areas compared to patients initiated on olanzapine (70.6\%), risperidone (71.8\%) and typical antipsychotics $(73.6 \%)$. There were no significant differences between the patients initiated on quetiapine and other antipsychotics based on gender and race. Significantly higher proportion of patients initiated on quetiapine (65.2\%) had a psychiatric prescriber for index prescription as compared to patients initiated on typical antipsychotic (34.0\%).

A significantly higher proportion of patients initiated on quetiapine showed a mixed diagnosis (68.9\%) as compared to patients initiated olanzapine (48.1\%), risperidone (32.5\%) or typical antipsychotics (46.3\%). There was significantly a higher proportion of patients with alcohol and substance abuse in the quetiapine cohort (21.6\%) as compared to the risperidone $(15.2 \%)$ cohort. There was significantly a lesser proportion of patients with major depression in the quetiapine cohort (13.3\%) as compared to the olanzapine (35.8\%), risperidone (29.4\%) or typical antipsychotics (21.3\%) cohorts. Also, there were significantly lesser proportion of patients with mild to moderate depression, anxiety and personality disorder in the quetiapine cohort as compared to the other antipsychotic cohorts. 
Table 16: Demographic characteristics of schizophrenia patients (18 years and older) who were initiated on study antipsychotics between January 1, 1999 and December 31, $2001(\mathrm{~N}=999)$

\begin{tabular}{|c|c|c|c|c|}
\hline & $\begin{array}{c}\text { Olanzapine } \\
N=346\end{array}$ & $\begin{array}{c}\text { Risperidone } \\
N=201\end{array}$ & $\begin{array}{c}\text { Quetiapine } \\
N=149\end{array}$ & $\begin{array}{c}\text { Typical } \\
N=303\end{array}$ \\
\hline \multicolumn{5}{|l|}{ Demographics } \\
\hline $\begin{array}{l}\text { Age in years } \\
(\text { Mean/포) }\end{array}$ & $40.0( \pm 11.8)$ & $38.8( \pm 14.2)$ & $41.0( \pm 11.5)$ & $43.9( \pm 11.0)$ \\
\hline \multicolumn{5}{|l|}{ Gender } \\
\hline Females & $\begin{array}{c}188 \\
(54.34 \%)\end{array}$ & $\begin{array}{c}106 \\
(53.00 \%)\end{array}$ & $\begin{array}{c}89 \\
(60.54 \%)\end{array}$ & $\begin{array}{c}166 \\
(55.15 \%)\end{array}$ \\
\hline Males & $\begin{array}{c}158 \\
(45.66 \%)\end{array}$ & $\begin{array}{c}94^{\mathrm{Q}} \\
(47.00 \%)\end{array}$ & $\begin{array}{c}58 \\
(39.46 \%)\end{array}$ & $\begin{array}{c}135 \\
(44.85 \%)\end{array}$ \\
\hline \multicolumn{5}{|l|}{ Race } \\
\hline Caucasians & $\begin{array}{c}322 \\
(93.06 \%)\end{array}$ & $\begin{array}{c}182 \\
(90.55 \%)\end{array}$ & $\begin{array}{c}137 \\
(91.95 \%)\end{array}$ & $\begin{array}{c}260 \\
(85.81 \%)\end{array}$ \\
\hline Others & $\begin{array}{c}24 \\
(6.94 \%)\end{array}$ & $\begin{array}{c}19 \\
(9.45 \%)\end{array}$ & $\begin{array}{c}12 \\
(8.05 \%)\end{array}$ & $\begin{array}{c}43 \\
(14.19 \%)\end{array}$ \\
\hline \multicolumn{5}{|l|}{ Residence } \\
\hline Metro & $\begin{array}{c}115^{Q} \\
(33.24 \%)\end{array}$ & $\begin{array}{c}48 \\
(23.88 \%)\end{array}$ & $\begin{array}{c}31 \text { OT } \\
(20.81 \%)\end{array}$ & $\begin{array}{c}98^{\mathrm{Q}} \\
(32.34 \%)\end{array}$ \\
\hline Non-Metro & $\begin{array}{c}231^{Q} \\
(66.76 \%)\end{array}$ & $\begin{array}{c}153 \\
(76.12 \%)\end{array}$ & $\begin{array}{c}118^{\text {OT }} \\
(79.19 \%)\end{array}$ & $\begin{array}{c}205^{Q} \\
(67.66 \%)\end{array}$ \\
\hline \multicolumn{5}{|c|}{ Prescriber type } \\
\hline Psychiatric & $\begin{array}{c}167^{Q} \\
(55.67 \%)\end{array}$ & $\begin{array}{c}87^{\mathrm{Q}} \\
(55.06 \%)\end{array}$ & $\begin{array}{c}84^{\text {ORT }} \\
(68.29 \%)\end{array}$ & $\begin{array}{c}94^{\mathrm{Q}} \\
(42.53 \%)\end{array}$ \\
\hline $\begin{array}{l}\text { Non- } \\
\text { Psychiatric }\end{array}$ & $\begin{array}{c}133^{Q} \\
(44.33 \%)\end{array}$ & $\begin{array}{c}71^{Q} \\
(44.94 \%)\end{array}$ & $\begin{array}{c}39^{\text {ORT }} \\
(31.97 \%)\end{array}$ & $\begin{array}{c}127^{\mathrm{Q}} \\
(57.47 \%)\end{array}$ \\
\hline \multicolumn{5}{|c|}{ Year of Index Prescription } \\
\hline 1999 & $\begin{array}{c}176^{Q} \\
(50.87 \%)\end{array}$ & $\begin{array}{c}105^{\mathrm{Q}} \\
(52.24 \%)\end{array}$ & $\begin{array}{c}54^{\text {ORT }} \\
(36.24 \%)\end{array}$ & $\begin{array}{c}219^{Q} \\
(72.28 \%)\end{array}$ \\
\hline 2000 & $\begin{array}{c}98 \\
(28.32 \%)\end{array}$ & $\begin{array}{c}59 \\
(29.35 \%)\end{array}$ & $\begin{array}{c}50^{\mathrm{T}} \\
(33.56 \%)\end{array}$ & $\begin{array}{c}55^{\mathrm{Q}} \\
(18.15 \%)\end{array}$ \\
\hline 2001 & $\begin{array}{c}72^{Q} \\
(20.81 \%)\end{array}$ & $\begin{array}{c}37^{\mathrm{Q}} \\
(18.41 \%)\end{array}$ & $\begin{array}{c}45^{\text {ORT }} \\
(30.20 \%)\end{array}$ & $\begin{array}{c}29^{Q} \\
(9.57 \%)\end{array}$ \\
\hline Schizophrenia & $\begin{array}{c}129 \\
(37.28 \%)\end{array}$ & $\begin{array}{c}71 \\
(35.32 \%)\end{array}$ & $\begin{array}{c}31 \\
(20.81 \%)\end{array}$ & $\begin{array}{c}150 \\
(49.50 \%)\end{array}$ \\
\hline
\end{tabular}


Table 16: Demographic characteristics of schizophrenia patients (18 years and older) who were initiated on study antipsychotics between January 1, 1999 and December 31, 2001 ( $\mathbf{N}=999)$ (contd.)

\begin{tabular}{|c|c|c|c|c|}
\hline & $\begin{array}{c}\text { Olanzapine } \\
N=346\end{array}$ & $\begin{array}{c}\text { Risperidone } \\
N=201\end{array}$ & $\begin{array}{c}\text { Quetiapine } \\
N=149\end{array}$ & $\begin{array}{l}\text { Typical } \\
N=303\end{array}$ \\
\hline \multicolumn{5}{|c|}{ Schizophrenia Subtype } \\
\hline $\begin{array}{l}\text { Schizoaffective } \\
\text { Disoders }\end{array}$ & $\begin{array}{c}80 \\
(23.12 \%)\end{array}$ & $\begin{array}{c}55 \\
(27.36 \%)\end{array}$ & $\begin{array}{c}32 \\
(21.48 \%)\end{array}$ & $\begin{array}{c}58 \\
(19.14 \%)\end{array}$ \\
\hline Mixed diagnosis & $\begin{array}{c}137^{\mathrm{Q}} \\
(39.60 \%)\end{array}$ & $\begin{array}{c}75^{Q} \\
(37.31 \%)\end{array}$ & $\begin{array}{c}86^{\mathrm{ORT}} \\
(57.72 \%)\end{array}$ & $\begin{array}{c}95^{\mathrm{Q}} \\
(31.35 \%)\end{array}$ \\
\hline \multicolumn{5}{|l|}{ Co-morbidities } \\
\hline $\begin{array}{l}\text { Alcohol and } \\
\text { substance abuse }\end{array}$ & $\begin{array}{c}77 \\
(22.25 \%)\end{array}$ & $\begin{array}{c}30^{\mathrm{Q}} \\
(14.93 \%)\end{array}$ & $\begin{array}{c}37^{\mathrm{R}} \\
(24.83 \%)\end{array}$ & $\begin{array}{c}70 \\
(23.10 \%)\end{array}$ \\
\hline Bipolar disorder & $\begin{array}{c}51 \\
(14.74 \%)\end{array}$ & $\begin{array}{c}28 \\
(13.93 \%)\end{array}$ & $\begin{array}{c}27 \\
(18.12 \%)\end{array}$ & $\begin{array}{c}36 \\
(11.88 \%)\end{array}$ \\
\hline $\begin{array}{l}\text { Major } \\
\text { Depression }\end{array}$ & $\begin{array}{c}80 \\
(23.12 \%)\end{array}$ & $\begin{array}{c}33 \\
(16.42 \%)\end{array}$ & $\begin{array}{c}27 \\
(18.12 \%)\end{array}$ & $\begin{array}{c}38 \\
(12.54 \%)\end{array}$ \\
\hline $\begin{array}{l}\text { Mild to } \\
\text { Moderate } \\
\text { Depression }\end{array}$ & $\begin{array}{c}55 \\
(15.90 \%)\end{array}$ & $\begin{array}{c}29 \\
(14.43 \%)\end{array}$ & $\begin{array}{c}20^{\mathrm{T}} \\
(13.42 \%)\end{array}$ & $\begin{array}{c}23^{Q} \\
(7.59 \%)\end{array}$ \\
\hline Anxiety & $\begin{array}{c}72 \\
(20.81 \%)\end{array}$ & $\begin{array}{c}26 \\
(12.94 \%)\end{array}$ & $\begin{array}{c}28 \\
(18.79 \%)\end{array}$ & $\begin{array}{c}43 \\
(14.19 \%)\end{array}$ \\
\hline $\begin{array}{l}\text { Personality } \\
\text { Disorder }\end{array}$ & $\begin{array}{c}40 \\
(11.56 \%)\end{array}$ & $\begin{array}{c}20 \\
(9.95 \%)\end{array}$ & $\begin{array}{c}18 \\
(12.08 \%)\end{array}$ & $\begin{array}{c}26 \\
(8.58 \%)\end{array}$ \\
\hline $\begin{array}{l}\text { Non- } \\
\text { Schizophrenia } \\
\text { Disorder }\end{array}$ & $\begin{array}{c}19 \\
(5.49 \%)\end{array}$ & $\begin{array}{c}7 \\
(3.48 \%)\end{array}$ & $\begin{array}{c}3 \\
(2.01 \%)\end{array}$ & $\begin{array}{c}8 \\
(2.64 \%)\end{array}$ \\
\hline $\begin{array}{l}\text { Other Affective } \\
\text { Disorders }\end{array}$ & $\begin{array}{c}7 \\
(2.02 \%)\end{array}$ & $\begin{array}{c}5 \\
(2.49 \%)\end{array}$ & $\begin{array}{c}4 \\
(2.68 \%)\end{array}$ & $\begin{array}{c}2 \\
(0.66 \%)\end{array}$ \\
\hline $\begin{array}{l}\text { Attention deficit } \\
\text { Disorder }\end{array}$ & $\begin{array}{c}8 \\
(2.31 \%)\end{array}$ & $\begin{array}{c}13 \\
(6.47 \%)\end{array}$ & $\begin{array}{c}3 \\
(2.01 \%)\end{array}$ & $\begin{array}{c}5 \\
(1.65 \%)\end{array}$ \\
\hline Diabetes & $\begin{array}{c}31 \\
(8.96 \%)\end{array}$ & $\begin{array}{c}21 \\
(10.45 \%)\end{array}$ & $\begin{array}{c}15 \\
(10.07 \%)\end{array}$ & $\begin{array}{c}40 \\
(13.20 \%)\end{array}$ \\
\hline Hyperlipedemia & $\begin{array}{c}24 \\
(6.94 \%)\end{array}$ & $\begin{array}{c}9 \\
(4.48 \%)\end{array}$ & $\begin{array}{c}16 \\
(10.74 \%)\end{array}$ & $\begin{array}{c}22 \\
(7.26 \%)\end{array}$ \\
\hline Hypertension & $\begin{array}{c}59 \\
(17.05 \%)\end{array}$ & $\begin{array}{c}35 \\
(17.41 \%)\end{array}$ & $\begin{array}{c}35 \\
(23.49 \%)\end{array}$ & $\begin{array}{c}49 \\
(16.17 \%)\end{array}$ \\
\hline
\end{tabular}


Table 16: Demographic characteristics of schizophrenia patients (18 years and older) who were initiated on study antipsychotics between January 1, 1999 and December 31, 2001 ( $\mathbf{N}=999)$ (contd.)

\begin{tabular}{lcccc}
\hline & $\begin{array}{c}\text { Olanzapine } \\
\boldsymbol{N}=\mathbf{3 4 6}\end{array}$ & $\begin{array}{c}\text { Risperidone } \\
\boldsymbol{N}=\mathbf{2 0 1}\end{array}$ & $\begin{array}{c}\text { Quetiapine } \\
\boldsymbol{N}=\mathbf{1 4 9}\end{array}$ & $\begin{array}{c}\text { Typical } \\
\boldsymbol{N}=\mathbf{3 0 3}\end{array}$ \\
\hline Charlson & 0.4 & 0.5 & 0.5 & 0.7 \\
$\begin{array}{l}\text { Comorbidity } \\
\text { Index }\end{array}$ & $(1.1)$ & $(1.4)$ & $(0.9)$ & $(1.6)$ \\
\hline
\end{tabular}

${ }^{\mathrm{O}}$ Significant difference between quetiapine and olanzapine cohorts at $\mathrm{p}<0.05$

${ }^{\mathrm{R}}$ Significant difference between quetiapine and risperidone cohorts at $\mathrm{p}<0.05$

${ }^{\mathrm{T}}$ Significant difference between quetiapine and typicals cohorts at $\mathrm{p}<0.05$ 
Table 17: Demographic characteristics of bipolar disorder patients who were initiated on study antipsychotics between January 1, 1999 and December 31, 2001 $(\mathbf{N}=\mathbf{8 2 5})$

\begin{tabular}{|c|c|c|c|c|}
\hline & $\begin{array}{c}\text { Olanzapine } \\
N=283\end{array}$ & $\begin{array}{c}\text { Risperidone } \\
N=231\end{array}$ & $\begin{array}{c}\text { Quetiapine } \\
N=106\end{array}$ & $\begin{array}{l}\text { Typical } \\
N=205\end{array}$ \\
\hline \multicolumn{5}{|l|}{ Age category } \\
\hline $\begin{array}{l}18 \text { years or } \\
\text { above }\end{array}$ & $\begin{array}{c}239 \\
(84.5 \%)\end{array}$ & $\begin{array}{c}146^{\mathrm{Q}} \\
(63.2 \%)\end{array}$ & $\begin{array}{c}96^{\mathrm{R}} \\
(90.6 \%)\end{array}$ & $\begin{array}{c}197 \\
(96.1 \%)\end{array}$ \\
\hline $\begin{array}{l}\text { Age in years } \\
\text { (Mean+SD) }\end{array}$ & $39.9( \pm 11.0)$ & $41.5( \pm 12.7)$ & $40.5( \pm 10.7)$ & $43.0( \pm 11.5)$ \\
\hline $\begin{array}{l}\text { Less than } 18 \\
\text { years }\end{array}$ & $\begin{array}{c}44 \\
(15.5 \%)\end{array}$ & $\begin{array}{c}85^{\mathrm{Q}} \\
(36.8 \%)\end{array}$ & $\begin{array}{c}10^{\mathrm{R}} \\
(9.4 \%)\end{array}$ & $\begin{array}{c}8 \\
(3.9 \%)\end{array}$ \\
\hline $\begin{array}{l}\text { Age in years } \\
\text { (Mean/_t_SD) }\end{array}$ & $12.8( \pm 3.3)$ & $11.1( \pm 3.4)$ & $14.2( \pm 2.9)$ & $13.1( \pm 2.7)$ \\
\hline \multicolumn{5}{|l|}{ Gender } \\
\hline Females & $\begin{array}{c}156 \\
(55.1 \%)\end{array}$ & $\begin{array}{c}123 \\
(53.2 \%)\end{array}$ & $\begin{array}{c}61 \\
(57.5 \%)\end{array}$ & $\begin{array}{c}116 \\
(56.6 \%)\end{array}$ \\
\hline Males & $\begin{array}{c}127 \\
(44.8 \%)\end{array}$ & $\begin{array}{c}107 \\
(46.3 \%)\end{array}$ & $\begin{array}{c}44 \\
(41.5 \%)\end{array}$ & $\begin{array}{c}87 \\
(42.4 \%)\end{array}$ \\
\hline \multicolumn{5}{|l|}{ Race } \\
\hline Caucasians & $264(93.3 \%)$ & $210(90.9 \%)$ & 97 (91.5\%) & $181(88.4 \%)$ \\
\hline Others & $19(6.7 \%)$ & $21(9.0 \%)$ & $9(8.5 \%)$ & $24(11.7 \%)$ \\
\hline \multicolumn{5}{|l|}{ Residence } \\
\hline Metro & $\begin{array}{c}83 \mathrm{Q} \\
(29.3 \%)\end{array}$ & $\begin{array}{c}65^{\mathrm{Q}} \\
(28.1 \%)\end{array}$ & $\begin{array}{c}17^{\text {ORT }} \\
(16.0 \%)\end{array}$ & $\begin{array}{c}54^{\mathrm{Q}} \\
(26.3 \%)\end{array}$ \\
\hline Non-Metro & $\begin{array}{c}200^{Q} \\
(70.6 \%)\end{array}$ & $\begin{array}{c}166^{Q} \\
(71.8 \%)\end{array}$ & $\begin{array}{c}89^{\text {ORT }} \\
(84.0 \%)\end{array}$ & $\begin{array}{c}151^{Q} \\
(73.6 \%)\end{array}$ \\
\hline \multicolumn{5}{|c|}{ Prescriber type } \\
\hline Psychiatric & $\begin{array}{c}135 \\
(60.0 \%)\end{array}$ & $\begin{array}{c}103 \\
(59.5 \%)\end{array}$ & $\begin{array}{c}60^{\mathrm{T}} \\
(65.2 \%)\end{array}$ & $\begin{array}{c}54^{\mathrm{Q}} \\
(34.0 \%)\end{array}$ \\
\hline $\begin{array}{l}\text { Non- } \\
\text { Psychiatric }\end{array}$ & $\begin{array}{c}90 \\
(40.0 \%)\end{array}$ & $\begin{array}{c}70 \\
(40.5 \%)\end{array}$ & $\begin{array}{c}32^{\mathrm{T}} \\
(34.9 \%)\end{array}$ & $\begin{array}{c}105 \mathrm{Q} \\
(66.0 \%)\end{array}$ \\
\hline
\end{tabular}


Table 17: Demographic characteristics of bipolar disorder patients who were initiated on study antipsychotics between January 1, 1999 and December 31, 2001 $(\mathbf{N}=\mathbf{8 2 5})$ (contd.)

\begin{tabular}{|c|c|c|c|c|}
\hline & $\begin{array}{c}\text { Olanzapine } \\
N=283\end{array}$ & $\begin{array}{c}\text { Risperidone } \\
N=231\end{array}$ & $\begin{array}{c}\text { Quetiapine } \\
N=106\end{array}$ & $\begin{array}{r}\text { Typical } \\
N=205 \\
\end{array}$ \\
\hline \multicolumn{5}{|c|}{ Year of Index Prescription } \\
\hline 1999 & $\begin{array}{c}137^{Q} \\
(48.4 \%)\end{array}$ & $\begin{array}{c}124^{Q} \\
(53.7 \%)\end{array}$ & $\begin{array}{c}36^{\mathrm{ORT}} \\
(34.0 \%)\end{array}$ & $\begin{array}{c}140^{\mathrm{Q}} \\
(68.3 \%)\end{array}$ \\
\hline 2000 & $\begin{array}{c}83^{Q} \\
(29.3 \%)\end{array}$ & $\begin{array}{c}65^{Q} \\
(28.1 \%)\end{array}$ & $\begin{array}{c}36 \\
(34.0 \%)\end{array}$ & $\begin{array}{c}45^{\mathrm{Q}} \\
(21.9 \%)\end{array}$ \\
\hline 2001 & $\begin{array}{c}63^{Q} \\
(22.3 \%)\end{array}$ & $\begin{array}{c}42^{Q} \\
(18.2 \%)\end{array}$ & $\begin{array}{c}344^{\mathrm{ORT}} \\
(32.1 \%)\end{array}$ & $\begin{array}{c}20^{\mathrm{Q}} \\
(9.8 \%)\end{array}$ \\
\hline \multicolumn{5}{|c|}{ Bipolar Disorder Subtype } \\
\hline Bipolar 1 & $\begin{array}{c}91^{\mathrm{Q}} \\
(32.2 \%)\end{array}$ & $\begin{array}{c}89^{Q} \\
(38.5 \%)\end{array}$ & $\begin{array}{c}13^{\mathrm{ORT}} \\
(12.3 \%)\end{array}$ & $\begin{array}{c}79^{\mathrm{Q}} \\
(38.5 \%)\end{array}$ \\
\hline Bipolar 2 & $\begin{array}{c}56 \\
(19.8 \%)\end{array}$ & $\begin{array}{c}67 \\
(29.0 \%)\end{array}$ & $\begin{array}{c}20 \\
(18.9 \%)\end{array}$ & $\begin{array}{c}31 \\
(15.1 \%)\end{array}$ \\
\hline Mixed & $\begin{array}{c}136^{\mathrm{Q}} \\
(48.1 \%)\end{array}$ & $\begin{array}{c}75^{Q} \\
(32.5 \%)\end{array}$ & $\begin{array}{c}73^{\text {ORT }} \\
(68.9 \%)\end{array}$ & $\begin{array}{c}95^{Q} \\
(46.3 \%)\end{array}$ \\
\hline \multicolumn{5}{|l|}{ Co-morbidities } \\
\hline $\begin{array}{l}\text { Alcohol and } \\
\text { substance abuse }\end{array}$ & $\begin{array}{c}27 \\
(25.5 \%)\end{array}$ & $\begin{array}{c}35^{Q} \\
(15.2 \%)\end{array}$ & $\begin{array}{c}61^{\mathrm{R}} \\
(21.6 \%)\end{array}$ & $\begin{array}{c}42 \\
(20.5 \%)\end{array}$ \\
\hline Schizophrenia & $\begin{array}{c}51 \\
(14.74 \%)\end{array}$ & $\begin{array}{c}28 \\
(13.93 \%)\end{array}$ & $\begin{array}{c}27 \\
(18.12 \%)\end{array}$ & $\begin{array}{c}36 \\
(11.88 \%)\end{array}$ \\
\hline $\begin{array}{l}\text { Major } \\
\text { Depression }\end{array}$ & $\begin{array}{c}67^{\mathrm{Q}} \\
(35.8 \%)\end{array}$ & $\begin{array}{c}55^{Q} \\
(29.4 \%)\end{array}$ & $\begin{array}{l}25^{\text {ORT }} \\
(13.3 \%)\end{array}$ & $\begin{array}{c}40 \\
(21.3 \%)\end{array}$ \\
\hline $\begin{array}{l}\text { Mild to Moderate } \\
\text { Depression }\end{array}$ & $\begin{array}{c}62^{\mathrm{Q}} \\
(36.2 \%)\end{array}$ & $\begin{array}{c}51^{\mathrm{Q}} \\
(29.8 \%)\end{array}$ & $\begin{array}{l}21^{\text {ORT }} \\
(12.2 \%)\end{array}$ & $\begin{array}{c}37 \\
(21.6 \%)\end{array}$ \\
\hline Anxiety & $\begin{array}{c}71^{\mathrm{Q}} \\
(39.2 \%)\end{array}$ & $\begin{array}{c}46^{\mathrm{Q}} \\
(25.4 \%)\end{array}$ & $\begin{array}{l}13^{\mathrm{ORT}} \\
(7.1 \%)\end{array}$ & $\begin{array}{c}51^{\mathrm{Q}} \\
(28.1 \%)\end{array}$ \\
\hline $\begin{array}{l}\text { Personality } \\
\text { Disorder }\end{array}$ & $\begin{array}{c}43^{\mathrm{Q}} \\
(34.9 \%)\end{array}$ & $\begin{array}{c}32^{Q} \\
(26.0 \%)\end{array}$ & $\begin{array}{c}15^{\text {ORT }} \\
(12.2 \%)\end{array}$ & $\begin{array}{c}33^{Q} \\
(26.8 \%)\end{array}$ \\
\hline $\begin{array}{l}\text { Non- } \\
\text { Schizophrenia } \\
\text { Disorder }\end{array}$ & $\begin{array}{c}8 \\
(24.2 \%)\end{array}$ & $\begin{array}{c}8 \\
(24.2 \%)\end{array}$ & $\begin{array}{c}6 \\
(18.1 \%)\end{array}$ & $\begin{array}{c}11 \\
(33.3 \%)\end{array}$ \\
\hline $\begin{array}{l}\text { Other Affective } \\
\text { Disorders }\end{array}$ & $\begin{array}{c}9 \\
(31.0 \%)\end{array}$ & $\begin{array}{c}8 \\
(27.5 \%)\end{array}$ & $\begin{array}{c}3 \\
(10.3 \%)\end{array}$ & $\begin{array}{c}9 \\
(31.0 \%)\end{array}$ \\
\hline
\end{tabular}


Table 17: Demographic characteristics of bipolar disorder patients who were initiated on study antipsychotics between January 1, 1999 and December 31, 2001 $(\mathrm{N}=\mathbf{8 2 5})$ (contd.)

\begin{tabular}{|c|c|c|c|c|}
\hline & $\begin{array}{c}\text { Olanzapine } \\
N=283\end{array}$ & $\begin{array}{c}\text { Risperidone } \\
N=231\end{array}$ & $\begin{array}{c}\text { Quetiapine } \\
N=106\end{array}$ & $\begin{array}{l}\text { Typical } \\
N=205\end{array}$ \\
\hline $\begin{array}{l}\text { Attention deficit } \\
\text { Disorder }\end{array}$ & $\begin{array}{c}39 \\
(34.2 \%)\end{array}$ & $\begin{array}{c}34 \\
(29.8 \%)\end{array}$ & $\begin{array}{c}17 \\
(14.9 \%)\end{array}$ & $\begin{array}{c}24 \\
(21.0 \%)\end{array}$ \\
\hline Diabetes & $\begin{array}{c}25 \\
(8.8 \%)\end{array}$ & $\begin{array}{c}24 \\
(10.4 \%)\end{array}$ & $\begin{array}{c}9 \\
(8.5 \%)\end{array}$ & $\begin{array}{c}28 \\
(13.6 \%)\end{array}$ \\
\hline Hyperlipidemia & $\begin{array}{c}17 \\
(6.0 \%)\end{array}$ & $\begin{array}{c}14 \\
(6.0 \%)\end{array}$ & $\begin{array}{c}9 \\
(8.5 \%)\end{array}$ & $\begin{array}{c}19 \\
(9.3 \%)\end{array}$ \\
\hline Hypertension & $\begin{array}{c}47 \\
(16.6 \%)\end{array}$ & $\begin{array}{c}42 \\
(18.2 \%)\end{array}$ & $\begin{array}{c}24 \\
(22.6 \%)\end{array}$ & $\begin{array}{c}40 \\
(19.5 \%)\end{array}$ \\
\hline $\begin{array}{l}\text { Charlson } \\
\text { Comorbidity } \\
\text { Index }\end{array}$ & $\begin{array}{l}0.52 \\
(1.1)\end{array}$ & $\begin{array}{l}0.54 \\
(1.5)\end{array}$ & $\begin{array}{l}0.59 \\
(0.9)\end{array}$ & $\begin{array}{c}0.9 \\
(1.9)\end{array}$ \\
\hline
\end{tabular}

${ }^{0}$ Significant difference between quetiapine and olanzapine cohorts at $p<0.05$

${ }^{\mathrm{R}}$ Significant difference between quetiapine and risperidone cohorts at $\mathrm{p}<0.05$

${ }^{\mathrm{T}}$ Significant difference between quetiapine and typicals cohorts at $\mathrm{p}<0.05$ 


\section{Results for research objective 8}

For schizophrenia and bipolar disorder patients, to compare the impact of quetiapine with risperidone, olanzapine, and typical antipsychotics on total and mental healthcare costs

$\underline{\text { Schizophrenia }}$

Results for univariate analysis comparing differences in total and mental costs between each of the index antipsychotics are reported in table 18 . There were no significant differences in total healthcare costs of quetiapine cohort compared to other index antipsychotic cohorts. Total post-index mental health-related costs for quetiapine $(\$ 15,318 \pm \$ 20,343)$ were significantly lesser compared to olanzapine $(\$ 17,919 \pm$ $\$ 25,660)$ and typical cohorts $(\$ 18,342 \pm \$ 26,730)$.

It is possible that various factors such as patient demographics, comorbidities, disease severity, or sample selection bias could have confounded the results of univariate analysis. Therefore, a multivariate analysis controlling for various confounders was conducted to determine the impact of index antipsychotic use on total healthcare costs.

The first step involved developing a multinomial logistic regression model predicting treatment selection. This model allowed for calculation of Inverse Mills Ratio (IMR) to adjust for sample selection bias in the second step regression models. Individual contrasts were carried out to obtain IMR for quetiapine vs. olanzapine, quetiapine vs. risperidone, and quetiapine vs. typicals. The results of this multinomial logistic regression model are presented in table 19.

In the second step of the analysis, three semi-log OLS regressions were developed to compare costs associated with olanzapine, risperidone and typical with quetiapine 
therapy. IMRs calculated from the first stage regression models were added as a covariate in the second stage regression models to adjust and test for sample selection bias. The second stage regression models are presented in tables 20a to $21 \mathrm{c}$. Variables were dropped from the model if data was sparse for a variable. Certain variables such as pre-index ECT, pre-index clozapine use, pre-index suicides, and pre-index arrhythmia were dropped due to sparse data. Variables were also dropped from the model if they were highly collinear with other variables. Variance inflation factors were less than 3 . Wherever needed, White's correction for heteroskedasity was used. 
Table 18: Healthcare costs comparison among schizophrenia patients: (Quetiapine versus other antipsychotics)

\begin{tabular}{|c|c|c|c|c|}
\hline & $\begin{array}{c}\text { Olanzapine } \\
N=346\end{array}$ & $\begin{array}{c}\text { Risperidone } \\
\quad N=201\end{array}$ & $\begin{array}{c}\text { Quetiapine } \\
N=149\end{array}$ & $\begin{array}{c}\text { Typical } \\
N=303\end{array}$ \\
\hline \multicolumn{5}{|c|}{ Total health-related Costs } \\
\hline \multicolumn{5}{|c|}{$\begin{array}{l}\text { Total } \\
\text { Mean }( \pm \text { std })\end{array}$} \\
\hline Pre-index & $\begin{array}{c}\$ 11,644 \\
( \pm \$ 18,638)\end{array}$ & $\begin{array}{c}\$ 14,486 \\
(+\$ 31,387)\end{array}$ & $\begin{array}{c}\$ 10,433 \\
(+\$ 11,451)\end{array}$ & $\begin{array}{c}\$ 9,908 \\
( \pm \$ 12,021)\end{array}$ \\
\hline Post-index & $\begin{array}{c}\$ 17,919 \\
( \pm \$ 25,660)\end{array}$ & $\begin{array}{c}\$ 19,488 \\
(+\$ 31,858)\end{array}$ & $\begin{array}{c}\$ 15,318 \\
(+\$ 20,343)\end{array}$ & $\begin{array}{c}\$ 18,342 \\
(+\$ 26,730)\end{array}$ \\
\hline \multicolumn{5}{|c|}{ Mental health-related Costs } \\
\hline \multicolumn{5}{|c|}{$\begin{array}{l}\text { Total Mental Health-related } \\
\text { Mean }( \pm \text { std })\end{array}$} \\
\hline Pre-index & $\begin{array}{c}\$ 7,342 \\
( \pm \$ 10,432)\end{array}$ & $\begin{array}{c}\$ 8,946 \\
(+\$ 24,339)\end{array}$ & $\begin{array}{c}\$ 6,965 \\
( \pm \$ 9,881)\end{array}$ & $\begin{array}{c}\$ 5,630 \\
( \pm \$ 8,429)\end{array}$ \\
\hline Post-index & $\begin{array}{l}\$ 12,281^{Q} \\
( \pm \$ 20,602)\end{array}$ & $\begin{array}{c}\$ 11,147 \\
( \pm \$ 16,709)\end{array}$ & $\begin{array}{c}\$ 9,638^{\mathrm{TO}} \\
( \pm \$ 12,893)\end{array}$ & $\begin{array}{r}\$ 10,265^{Q} \\
( \pm \$ 18,717)\end{array}$ \\
\hline \multicolumn{5}{|c|}{$\begin{array}{l}\text { Inpatient } \\
\text { Mean }( \pm \text { std })\end{array}$} \\
\hline Pre-index & $\begin{array}{c}\$ 4,476 \\
( \pm \$ 8,465)\end{array}$ & $\begin{array}{c}\$ 4,832 \\
(+\$ 22,435)\end{array}$ & $\begin{array}{c}\$ 4,478^{\mathrm{T}} \\
( \pm \$ 7,980)\end{array}$ & $\begin{array}{l}\$ 2,684^{Q} \\
( \pm \$ 6,221)\end{array}$ \\
\hline Post-index & $\begin{array}{c}\$ 4,813 \\
(+\$ 11,309)\end{array}$ & $\begin{array}{c}\$ 4,274 \\
( \pm \$ 11,285)\end{array}$ & $\begin{array}{c}\$ 4,865^{\mathrm{T}} \\
( \pm \$ 11,332)\end{array}$ & $\begin{array}{c}\$ 5,418 \text { Q } \\
( \pm \$ 15,787)\end{array}$ \\
\hline \multicolumn{5}{|c|}{$\begin{array}{l}\text { Outpatient } \\
\text { Mean }(+ \text { std })\end{array}$} \\
\hline Pre-index & $\begin{array}{c}\$ 2,343 \\
( \pm \$ 4,877)\end{array}$ & $\begin{array}{c}\$ 3,583 \\
( \pm \$ 9,096)\end{array}$ & $\begin{array}{c}\$ 2,021 \\
( \pm \$ 4,795)\end{array}$ & $\begin{array}{c}\$ 2,469 \\
( \pm \$ 4,718)\end{array}$ \\
\hline Post-index & $\begin{array}{c}\$ 4,136^{Q} \\
( \pm \$ 16,349)\end{array}$ & $\begin{array}{c}\$ 4247 \\
(+\$ 10,209)\end{array}$ & $\begin{array}{c}\$ 2,071^{\mathrm{O}} \\
( \pm \$ 3,479)\end{array}$ & $\begin{array}{c}\$ 3,218 \\
( \pm \$ 5,652)\end{array}$ \\
\hline \multicolumn{5}{|c|}{$\begin{array}{l}\text { ER } \\
\text { Mean }( \pm \text { std })\end{array}$} \\
\hline Pre-index & $\begin{array}{l}\$ 143 \\
(\$ 501)\end{array}$ & $\begin{array}{c}\$ 114 \\
(\$ 399)\end{array}$ & $\begin{array}{c}\$ 98^{\mathrm{T}} \\
(\$ 241)\end{array}$ & $\begin{array}{l}\$ 54^{\mathrm{Q}} \\
(\$ 224)\end{array}$ \\
\hline Post-index & $\begin{array}{c}\$ 259 \\
(\$ 1,396)\end{array}$ & $\begin{array}{l}\$ 152 \mathrm{Q} \\
(\$ 415)\end{array}$ & $\begin{array}{l}\$ 231^{\mathrm{R}} \\
(\$ 539)\end{array}$ & $\begin{array}{l}\$ 207 \\
(\$ 546)\end{array}$ \\
\hline
\end{tabular}


Table 18: Healthcare costs comparison among schizophrenia patients: (Quetiapine versus other antipsychotics) (contd.)

\begin{tabular}{lcccc}
\hline & $\begin{array}{c}\text { Olanzapine } \\
\mathbf{N = 3 4 6}\end{array}$ & $\begin{array}{c}\text { Risperidone } \\
\mathbf{N}=\mathbf{2 0 1}\end{array}$ & $\begin{array}{c}\text { Quetiapine } \\
\mathbf{N}=\mathbf{1 4 9}\end{array}$ & $\begin{array}{c}\text { Typical } \\
\mathbf{N}=\mathbf{3 0 3}\end{array}$ \\
\hline $\begin{array}{l}\text { Prescription } \\
\text { Mean }( \pm \mathbf{s t d})\end{array}$ & & & & \\
Pre-index & $\$ 379$ & $\$ 416$ & $\$ 367$ & $\$ 421$ \\
& $(\$ 658)$ & $(\$ 698)$ & $(\$ 590)$ & $(\$ 800)$ \\
Post-index & $\$ 3,072^{\mathrm{Q}}$ & $\$ 2,473$ & $\$ 2,470^{\mathrm{OT}}$ & $\$ 1,421^{\mathrm{Q}}$ \\
& $(\$ 2,285)$ & $(\$ 2,071)$ & $(\$ 1,789)$ & $(\$ 1,898)$ \\
\hline
\end{tabular}

\footnotetext{
${ }^{0}$ Significant difference between quetiapine and olanzapine cohorts at $\mathrm{p}<0.05$

${ }^{\mathrm{R}}$ Significant difference between quetiapine and risperidone cohorts at $\mathrm{p}<0.05$

${ }^{\mathrm{T}}$ Significant difference between quetiapine and typicals cohorts at $\mathrm{p}<0.05$
} 
Table 19: Multinomial logistic regression model determining predictors of index antipsychotic use among schizophrenia patients (first-stage sample selection model)

\begin{tabular}{|c|c|c|c|c|c|c|}
\hline & \multicolumn{2}{|c|}{$\begin{array}{l}\text { Olanzapine vs. } \\
\text { Quetiapine }\end{array}$} & \multicolumn{2}{|c|}{$\begin{array}{c}\text { Risperidone vs. } \\
\text { Quetiapine }\end{array}$} & \multicolumn{2}{|c|}{$\begin{array}{l}\text { Typicals vs. } \\
\text { Quetiapine }\end{array}$} \\
\hline & Coefficient & t-ratio & Coefficient & t-ratio & Coefficient & t-ratio \\
\hline \multicolumn{7}{|c|}{ Demographic characteristics } \\
\hline Age (in years) & 0.004 & 0.418 & -0.004 & -0.324 & 0.033 & $3.161^{*}$ \\
\hline Males (ref: females) & 0.088 & 0.581 & 0.002 & 1.204 & 0.001 & 0.877 \\
\hline $\begin{array}{l}\text { Whites (ref: non- } \\
\text { whites) }\end{array}$ & 0.230 & 0.592 & -0.069 & -0.168 & -0.413 & -1.069 \\
\hline $\begin{array}{l}\text { Metro (ref: non- } \\
\text { metro) }\end{array}$ & 0.211 & 0.710 & -0.435 & -1.335 & -0.044 & -0.141 \\
\hline \multicolumn{7}{|c|}{ Prescribing physician type } \\
\hline Psychiatric prescriber & $-0.001^{*}$ & $-3.805^{*}$ & -0.001 & $-3.908^{*}$ & -0.001 & $-3.95^{*}$ \\
\hline \multicolumn{7}{|c|}{ Schizophrenia subtype (ref: only schizophrenia) } \\
\hline $\begin{array}{l}\text { Schizoaffective } \\
\text { disorder }\end{array}$ & -0.402 & -1.303 & -0.248 & -0.746 & -0.860 & $-2.619^{*}$ \\
\hline $\begin{array}{l}\text { Both schizoaffective } \\
\text { disorder and } \\
\text { schizophrenia }\end{array}$ & -0.709 & $-2.289^{*}$ & -1.105 & $-3.284^{*}$ & -1.152 & $-3.532^{*}$ \\
\hline \multicolumn{7}{|c|}{ Pre-index co-morbidities } \\
\hline Diabetes & 0.123 & 0.321 & 0.418 & 1.001 & 0.627 & 1.604 \\
\hline Hyperlipidemia & -0.395 & -1.021 & -0.906 & -1.879 & -0.105 & -0.256 \\
\hline Hypertension & -0.242 & -0.837 & 0.078 & 0.240 & -0.133 & -0.420 \\
\hline $\begin{array}{l}\text { Alcohol and substance } \\
\text { abuse }\end{array}$ & -0.402 & -1.571 & -0.868 & $-2.859^{*}$ & -0.068 & -0.250 \\
\hline Bipolar disorder & -0.277 & -0.942 & -0.347 & -1.033 & -0.054 & -0.166 \\
\hline Major depression & 0.307 & 1.097 & -0.064 & -0.199 & 0.012 & 0.037 \\
\hline Anxiety disorder & -0.081 & -0.287 & -0.525 & -1.560 & -0.090 & -0.286 \\
\hline Personality disorder & 0.024 & 0.070 & -0.257 & -0.670 & -0.331 & -0.887 \\
\hline $\begin{array}{l}\text { Non-specified } \\
\text { psychosis }\end{array}$ & 0.931 & 1.384 & 0.925 & 1.227 & 0.312 & 0.418 \\
\hline $\begin{array}{l}\text { Other affective } \\
\text { psychosis }\end{array}$ & -0.477 & -0.685 & -0.122 & -0.161 & -0.866 & -0.936 \\
\hline
\end{tabular}


Table 19: Multinomial logistic regression model determining predictors of index antipsychotic use among schizophrenia patients (first-stage sample selection model) (contd.)

\begin{tabular}{|c|c|c|c|c|c|c|}
\hline & \multicolumn{2}{|c|}{$\begin{array}{l}\text { Olanzapine vs. } \\
\text { Quetiapine }\end{array}$} & \multicolumn{2}{|c|}{$\begin{array}{c}\text { Risperidone vs. } \\
\text { Quetiapine }\end{array}$} & \multicolumn{2}{|c|}{$\begin{array}{l}\text { Typicals vs. } \\
\text { Quetiapine }\end{array}$} \\
\hline & Coefficient & t-ratio & Coefficient & t-ratio & Coefficient & t-ratio \\
\hline $\begin{array}{l}\text { Attention deficit } \\
\text { disorders }\end{array}$ & -0.041 & -0.055 & 1.082 & 1.504 & 0.653 & 0.803 \\
\hline $\begin{array}{l}\text { Mild to moderate } \\
\text { depression }\end{array}$ & 0.110 & 0.336 & 0.415 & 1.142 & -0.217 & -0.575 \\
\hline \multicolumn{7}{|c|}{ Pre-index healthcare utilization and cost } \\
\hline $\begin{array}{l}\text { Number of pre-index } \\
\text { mental-health related } \\
\text { ER visits }\end{array}$ & 0.000 & 1.196 & 0.000 & 1.111 & 0.184 & 0.045 \\
\hline $\begin{array}{l}\text { Number of pre-index } \\
\text { mental-health related } \\
\text { hospitalizations }\end{array}$ & 0.064 & 0.574 & -0.025 & -0.199 & -0.176 & -1.287 \\
\hline $\begin{array}{l}\text { Number of } \\
\text { psychotherapy visits }\end{array}$ & 0.014 & 0.651 & -0.019 & -0.558 & -0.001 & -0.050 \\
\hline $\begin{array}{l}\text { Number of medication } \\
\text { management visits }\end{array}$ & -0.064 & $-2.024^{*}$ & -0.047 & -1.285 & -0.095 & $-2.509^{*}$ \\
\hline \multicolumn{7}{|c|}{ Year of index prescription (ref: 1999) } \\
\hline - 2000 & -0.102 & -0.371 & 0.364 & 1.169 & -0.524 & -1.760 \\
\hline - 2001 & -0.312 & -1.051 & 0.054 & 0.158 & -1.130 & $-3.326^{*}$ \\
\hline \multicolumn{7}{|c|}{ Pre-index period psychiatric medication use ( days of supply) } \\
\hline Mood stabilizers & 0.001 & 0.710 & 0.000 & 0.125 & -0.002 & -1.991 \\
\hline Anticholinergics & -0.005 & -1.131 & -0.021 & -1.547 & -0.010 & -1.214 \\
\hline $\begin{array}{l}\text { Anxiolytics/hypnotics/ } \\
\text { sedatives }\end{array}$ & 0.001 & 0.755 & 0.002 & 1.015 & 0.001 & 0.802 \\
\hline Antipsychotics & -0.001 & -0.101 & 0.018 & $3.077^{*}$ & $0.0164^{*}$ & $2.937^{*}$ \\
\hline Antidepressants & 0.000 & 0.268 & 0.000 & -0.542 & -0.001 & -1.086 \\
\hline Benzodiazepines & -0.002 & $-2.829^{*}$ & -0.002 & -1.860 & -0.002 & $-2.046^{*}$ \\
\hline Antiparkinsons & -0.002 & -0.756 & -0.006 & -1.466 & 0.001 & 0.243 \\
\hline
\end{tabular}

*significant at $\mathrm{p}<0.05$

Model fit statistics:

Pseudo R-square $=12.43 \%$

-2 Log Likelihood $=1128.21 ; \chi^{2}=331.90 ; p=0.00$ 
Table 20a: Ordinary Least Squares (OLS) regression model for the impact of index antipsychotic on total healthcare cost among schizophrenia patients (second stage sample selection models): Olanzapine vs. quetiapine

\begin{tabular}{|c|c|c|c|c|}
\hline & Beta & S.E & t-statistic & Significance (p) \\
\hline \multicolumn{5}{|l|}{ Demographic characteristics } \\
\hline Age (in years) & 0.005 & 0.004 & 1.386 & 0.166 \\
\hline Males (ref: females) & -0.979 & 0.001 & -0.130 & 0.896 \\
\hline Whites (ref: non-whites) & 0.089 & 0.161 & 0.552 & 0.582 \\
\hline Metro (ref: non-metro) & 0.005 & 0.114 & 0.044 & 0.965 \\
\hline \multicolumn{5}{|l|}{ Prescribing physician type } \\
\hline Psychiatric prescriber & 0.000 & 0.000 & 2.702 & 0.007 \\
\hline \multicolumn{5}{|c|}{ Schizophrenia subtype (ref: only schizophrenia) } \\
\hline Schizoaffective disorder & -0.064 & 0.120 & -0.536 & 0.592 \\
\hline $\begin{array}{l}\text { Both schizoaffective disorder } \\
\text { and schizophrenia }\end{array}$ & -0.112 & 0.137 & -0.817 & 0.414 \\
\hline \multicolumn{5}{|l|}{ Pre-index co-morbidities } \\
\hline Charlson Comorbidity Index & 0.150 & 0.045 & 3.341 & 0.001 \\
\hline Alcohol and substance abuse & 0.257 & 0.103 & 2.492 & 0.013 \\
\hline Bipolar disorder & 0.206 & 0.135 & 1.527 & 0.128 \\
\hline Major depression & -0.016 & 0.108 & -0.144 & 0.886 \\
\hline Other mental comorbidities & -0.020 & 0.100 & -0.201 & 0.841 \\
\hline \multicolumn{5}{|c|}{ Pre-index healthcare utilization and cost } \\
\hline $\begin{array}{l}\text { Pre-index total health related } \\
\text { cost }\end{array}$ & 0.174 & 0.032 & 5.413 & 0.000 \\
\hline $\begin{array}{l}\text { Pre-index total health related } \\
\text { ER visits }\end{array}$ & -0.121 & 0.128 & -0.941 & 0.347 \\
\hline $\begin{array}{l}\text { Pre-index total health related } \\
\text { hospitalizations }\end{array}$ & 0.006 & 0.108 & 0.051 & 0.960 \\
\hline $\begin{array}{l}\text { Pre-index total health related } \\
\text { physician visits }\end{array}$ & 0.005 & 0.006 & 0.786 & 0.432 \\
\hline \multicolumn{5}{|c|}{ Year of index prescription (ref: 1999) } \\
\hline - 2000 & -0.093 & 0.117 & -0.795 & 0.427 \\
\hline - 2001 & -0.278 & 0.137 & -2.034 & 0.043 \\
\hline
\end{tabular}


Table 20a: Ordinary Least Squares (OLS) regression model for the impact of index antipsychotic on total healthcare cost among schizophrenia patients (second stage sample selection models): Olanzapine vs. quetiapine (contd.)

\begin{tabular}{lcccc}
\hline & Beta & S.E & t-statistic & Significance (p) \\
\hline Antipsychotics & 0.003 & 0.002 & 1.739 & 0.083 \\
Antidepressants & 0.000 & 0.000 & 1.001 & 0.317 \\
Benzodiazepines & 0.179 & 0.104 & 1.717 & 0.087 \\
Index prescription (ref: quetiapine) & & & \\
Olanzapine & 0.499 & 0.442 & 1.129 & 0.259 \\
Inverse Mills Ratio & -0.211 & 0.267 & -0.787 & 0.432 \\
\hline
\end{tabular}

\section{Model fit statistics:}

Adjusted R-square $=22.14 \%, \mathbf{F}=6.85, \mathbf{p}=0.00$ 
Table 20b: Ordinary Least Squares (OLS) regression model for the impact of index antipsychotic on total healthcare cost among schizophrenia patients (second stage sample selection models): Risperidone vs. quetiapine

\begin{tabular}{|c|c|c|c|c|}
\hline & Beta & S.E & t-statistic & Significance (p) \\
\hline \multicolumn{5}{|l|}{ Demographic characteristics } \\
\hline Age (in years) & 0.012 & 0.005 & 2.403 & 0.017 \\
\hline Males (ref: females) & 0.000 & 0.001 & 0.363 & 0.717 \\
\hline Whites (ref: non-whites) & 0.360 & 0.192 & 1.878 & 0.061 \\
\hline Metro (ref: non-metro) & -0.119 & 0.148 & -0.805 & 0.422 \\
\hline \multicolumn{5}{|l|}{ Prescribing physician type } \\
\hline Psychiatric prescriber & 0.000 & 0.000 & 1.184 & 0.238 \\
\hline \multicolumn{5}{|c|}{ Schizophrenia subtype (ref: only schizophrenia) } \\
\hline Schizoaffective disorder & 0.077 & 0.153 & 0.505 & 0.614 \\
\hline $\begin{array}{l}\text { Both schizoaffective disorder } \\
\text { and schizophrenia }\end{array}$ & -0.242 & 0.182 & -1.332 & 0.184 \\
\hline \multicolumn{5}{|l|}{ Pre-index co-morbidities } \\
\hline Charlson Comorbidity Index & 0.162 & 0.050 & 3.219 & 0.001 \\
\hline Alcohol and substance abuse & 0.346 & 0.160 & 2.170 & 0.031 \\
\hline Bipolar disorder & 0.229 & 0.171 & 1.336 & 0.183 \\
\hline Major depression & -0.009 & 0.150 & -0.057 & 0.954 \\
\hline Other mental comorbidities & -0.100 & 0.131 & -0.764 & 0.446 \\
\hline \multicolumn{5}{|c|}{ Pre-index healthcare utilization and cost } \\
\hline $\begin{array}{l}\text { Pre-index total health related } \\
\text { cost }\end{array}$ & 0.091 & 0.021 & 4.295 & 0.000 \\
\hline $\begin{array}{l}\text { Pre-index total health related } \\
\text { ER visits }\end{array}$ & 0.211 & 0.166 & 1.272 & 0.204 \\
\hline $\begin{array}{l}\text { Pre-index total health related } \\
\text { hospitalizations }\end{array}$ & -0.012 & 0.130 & -0.092 & 0.927 \\
\hline $\begin{array}{l}\text { Pre-index total health related } \\
\text { physician visits }\end{array}$ & 0.004 & 0.009 & 0.422 & 0.673 \\
\hline \multicolumn{5}{|c|}{ Year of index prescription (ref: 1999) } \\
\hline - 2000 & -0.199 & 0.153 & -1.295 & 0.196 \\
\hline - 2001 & -0.506 & 0.177 & -2.859 & 0.005 \\
\hline
\end{tabular}


Table 20b: Ordinary Least Squares (OLS) regression model for the impact of index antipsychotic on total healthcare cost among schizophrenia patients (second stage sample selection models): Risperidone vs. quetiapine (contd.)

\begin{tabular}{lcccc}
\hline \multicolumn{1}{c}{ Beta } & S.E & t-statistic & \multicolumn{2}{c}{$\begin{array}{l}\text { Significance } \\
(\boldsymbol{p})\end{array}$} \\
\hline Pre-index period psychiatric medication use ( days of supply) & & \\
Mood stabilizers & 0.001 & 0.000 & 2.834 & 0.005 \\
Antipsychotics & -0.001 & 0.002 & -0.666 & 0.506 \\
Antidepressants & 0.001 & 0.000 & 2.197 & 0.029 \\
Benzodiazepines & 0.100 & 0.133 & 0.752 & 0.453 \\
Index prescription (ref: quetiapine) & & & & \\
Risperidone & 0.207 & 0.394 & 0.526 & 0.599 \\
Inverse Mills Ratio & -0.040 & 0.246 & -0.161 & 0.873 \\
\hline
\end{tabular}

Model fit statistics:

Adjusted R-square $=27.5 \%, \mathbf{F}=6.52, \mathbf{p}=0.00$ 
Table 20c: Ordinary Least Squares (OLS) regression model for the impact of index antipsychotic on total healthcare cost among schizophrenia patients (second stage sample selection models): Typicals vs. quetiapine

\begin{tabular}{|c|c|c|c|c|}
\hline & Beta & S.E & t-statistic & Significance (p) \\
\hline \multicolumn{5}{|l|}{ Demographic characteristics } \\
\hline Age (in years) & 0.004 & 0.005 & 0.700 & 0.485 \\
\hline Males (ref: females) & 0.000 & 0.001 & 0.440 & 0.661 \\
\hline Whites (ref: non-whites) & 0.166 & 0.163 & 1.021 & 0.308 \\
\hline Metro (ref: non-metro) & -0.228 & 0.140 & -1.626 & 0.105 \\
\hline \multicolumn{5}{|l|}{ Prescribing physician type } \\
\hline Psychiatric prescriber & 0.000 & 0.000 & 1.428 & 0.154 \\
\hline \multicolumn{5}{|c|}{ Schizophrenia subtype (ref: only schizophrenia) } \\
\hline Schizoaffective disorder & 0.152 & 0.164 & 0.929 & 0.354 \\
\hline $\begin{array}{l}\text { Both schizoaffective disorder } \\
\text { and schizophrenia }\end{array}$ & -0.076 & 0.177 & -0.426 & 0.670 \\
\hline \multicolumn{5}{|l|}{ Pre-index co-morbidities } \\
\hline Charlson Comorbidity Index & 0.208 & 0.045 & 4.631 & 0.000 \\
\hline Alcohol and substance abuse & 0.255 & 0.133 & 1.913 & 0.057 \\
\hline Bipolar disorder & -0.107 & 0.184 & -0.580 & 0.562 \\
\hline Major depression & 0.071 & 0.154 & 0.463 & 0.644 \\
\hline Other mental comorbidities & 0.188 & 0.133 & 1.416 & 0.157 \\
\hline \multicolumn{5}{|c|}{ Pre-index healthcare utilization and cost } \\
\hline $\begin{array}{l}\text { Pre-index total health related } \\
\text { cost }\end{array}$ & 0.238 & 0.053 & 4.478 & 0.000 \\
\hline $\begin{array}{l}\text { Pre-index total health related } \\
\text { ER visits }\end{array}$ & 0.291 & 0.184 & 1.581 & 0.115 \\
\hline $\begin{array}{l}\text { Pre-index total health related } \\
\text { hospitalizations }\end{array}$ & 0.146 & 0.153 & 0.957 & 0.339 \\
\hline $\begin{array}{l}\text { Pre-index total health related } \\
\text { physician visits }\end{array}$ & 0.000 & 0.009 & 0.030 & 0.976 \\
\hline \multicolumn{5}{|c|}{ Year of index prescription (ref: 1999) } \\
\hline - 2000 & -0.064 & 0.173 & -0.369 & 0.712 \\
\hline - 2001 & -0.311 & 0.227 & -1.370 & 0.172 \\
\hline
\end{tabular}


Table 20c: Ordinary Least Squares (OLS) regression model for the impact of index antipsychotic on total healthcare cost among schizophrenia patients (second stage sample selection models): Typicals vs. quetiapine (contd.)

\begin{tabular}{lrrrr}
\hline & Beta & S.E & t-statistic & Significance (p) \\
\hline Mood stabilizers & 0.021 & 0.000 & 2.153 & 0.032 \\
Antipsychotics & -0.011 & 0.001 & -0.441 & 0.659 \\
Antidepressants & 0.000 & 0.000 & 0.853 & 0.394 \\
Benzodiazepines & 0.171 & 0.135 & 1.268 & 0.205 \\
Index prescription (ref: quetiapine) & & & & \\
Typical & -0.355 & 0.000 & -0.077 & 0.939 \\
Inverse Mills Ratio & -0.004 & 0.279 & -0.016 & 0.987 \\
\hline
\end{tabular}

\section{Model fit statistics:}

Adjusted R-square $=27.92 \%, F=8.28, p=0.00$ 
Table 21a: Ordinary Least Squares (OLS) regression model for the impact of index antipsychotic on mental healthcare cost among schizophrenia patients (second stage sample selection models): Olanzapine vs. quetiapine

\begin{tabular}{|c|c|c|c|c|}
\hline & Beta & S.E & t-statistic & Significance (p) \\
\hline \multicolumn{5}{|l|}{ Demographic characteristics } \\
\hline Age (in years) & 0.004 & 0.004 & 1.035 & 0.301 \\
\hline Males (ref: females) & 0.001 & 0.001 & 1.235 & 0.218 \\
\hline Whites (ref: non-whites) & 0.173 & 0.175 & 0.991 & 0.322 \\
\hline Metro (ref: non-metro) & -0.092 & 0.124 & -0.739 & 0.460 \\
\hline \multicolumn{5}{|l|}{ Prescribing physician type } \\
\hline Psychiatric prescriber & 0.000 & 0.000 & 2.592 & 0.010 \\
\hline \multicolumn{5}{|c|}{ Schizophrenia subtype (ref: only schizophrenia) } \\
\hline Schizoaffective disorder & -0.101 & 0.130 & -0.773 & 0.440 \\
\hline $\begin{array}{l}\text { Both schizoaffective disorder } \\
\text { and schizophrenia }\end{array}$ & -0.337 & 0.150 & -2.251 & 0.025 \\
\hline \multicolumn{5}{|l|}{ Pre-index co-morbidities } \\
\hline Charlson Comorbidity Index & 0.108 & 0.047 & 2.301 & 0.022 \\
\hline Alcohol and substance abuse & 0.365 & 0.112 & 3.256 & 0.001 \\
\hline Bipolar disorder & 0.095 & 0.147 & 0.647 & 0.518 \\
\hline Major depression & 0.041 & 0.117 & 0.345 & 0.730 \\
\hline Other mental comorbidities & 0.053 & 0.109 & 0.488 & 0.626 \\
\hline \multicolumn{5}{|c|}{ Pre-index healthcare utilization and cost } \\
\hline $\begin{array}{l}\text { Pre-index mental-health } \\
\text { related cost }\end{array}$ & 0.071 & 0.016 & 4.533 & 0.000 \\
\hline $\begin{array}{l}\text { Pre-index mental-health } \\
\text { related ER visits }\end{array}$ & -0.212 & 0.135 & -1.568 & 0.118 \\
\hline $\begin{array}{l}\text { Pre-index mental-health } \\
\text { related hospitalizations }\end{array}$ & 0.202 & 0.112 & 1.798 & 0.073 \\
\hline $\begin{array}{l}\text { Pre-index mental-health } \\
\text { related physician visits }\end{array}$ & -0.024 & 0.027 & -0.894 & 0.372 \\
\hline \multicolumn{5}{|c|}{ Year of index prescription (ref: 1999) } \\
\hline - 2000 & -0.035 & 0.127 & -0.272 & 0.786 \\
\hline - 2001 & -0.180 & 0.147 & -1.223 & 0.222 \\
\hline
\end{tabular}


Table 21a: Ordinary Least Squares (OLS) regression model for the impact of index antipsychotic on mental healthcare cost among schizophrenia patients (second stage sample selection models): Olanzapine vs. quetiapine (contd.)

\begin{tabular}{lcccc}
\hline & Beta & S.E & t-statistic & Significance (p) \\
\hline Pre-index period psychiatric medication use ( days of supply) & & \\
Mood stabilizers & 0.001 & 0.000 & 1.472 & 0.142 \\
Antipsychotics & 0.004 & 0.002 & 1.870 & 0.062 \\
Antidepressants & 0.000 & 0.000 & 1.207 & 0.228 \\
Benzodiazepines & 0.126 & 0.114 & 1.104 & 0.270 \\
Index prescription (ref: quetiapine) & & & & \\
Olanzapine & 0.482 & 0.478 & 1.008 & 0.314 \\
Inverse Mills Ratio & -0.169 & 0.290 & -0.583 & 0.560 \\
\hline
\end{tabular}

\section{Model fit statistics:}

Adjusted R-square $=19.97 \%, \mathbf{F}=6.14, \mathbf{p}=0.00$ 
Table 21b: Ordinary Least Squares (OLS) regression model for the impact of index antipsychotic on mental healthcare cost among schizophrenia patients (second stage sample selection models): Risperidone vs. quetiapine

\begin{tabular}{|c|c|c|c|c|}
\hline & Beta & S.E & t-statistic & Significance (p) \\
\hline \multicolumn{5}{|l|}{ Demographic characteristics } \\
\hline Age (in years) & 0.011 & 0.005 & 1.966 & 0.050 \\
\hline Males (ref: females) & 0.001 & 0.001 & 0.986 & 0.325 \\
\hline Whites (ref: non-whites) & 0.304 & 0.218 & 1.393 & 0.165 \\
\hline Metro (ref: non-metro) & -0.150 & 0.167 & -0.901 & 0.368 \\
\hline \multicolumn{5}{|l|}{ Prescribing physician type } \\
\hline Psychiatric prescriber & 0.000 & 0.000 & 1.645 & 0.101 \\
\hline \multicolumn{5}{|c|}{ Schizophrenia subtype (ref: only schizophrenia) } \\
\hline Schizoaffective disorder & -0.059 & 0.174 & -0.340 & 0.734 \\
\hline $\begin{array}{l}\text { Both schizoaffective disorder } \\
\text { and schizophrenia }\end{array}$ & -0.465 & 0.206 & -2.255 & 0.025 \\
\hline \multicolumn{5}{|l|}{ Pre-index co-morbidities } \\
\hline Charlson Comorbidity Index & 0.035 & 0.055 & 0.640 & 0.523 \\
\hline Alcohol and substance abuse & 0.547 & 0.181 & 3.029 & 0.003 \\
\hline Bipolar disorder & 0.228 & 0.194 & 1.173 & 0.242 \\
\hline Major depression & 0.056 & 0.170 & 0.331 & 0.741 \\
\hline Other mental comorbidities & -0.013 & 0.150 & -0.088 & 0.930 \\
\hline \multicolumn{5}{|c|}{ Pre-index healthcare utilization and cost } \\
\hline $\begin{array}{l}\text { Pre-index mental-health } \\
\text { related cost }\end{array}$ & 0.045 & 0.017 & 2.611 & 0.010 \\
\hline $\begin{array}{l}\text { Pre-index mental-health } \\
\text { related ER visits }\end{array}$ & 0.171 & 0.178 & 0.959 & 0.338 \\
\hline $\begin{array}{l}\text { Pre-index mental-health } \\
\text { related hospitalizations }\end{array}$ & 0.017 & 0.150 & 0.112 & 0.911 \\
\hline $\begin{array}{l}\text { Pre-index mental-health } \\
\text { related physician visits }\end{array}$ & -0.009 & 0.040 & -0.224 & 0.823 \\
\hline \multicolumn{5}{|c|}{ Year of index prescription (ref: 1999) } \\
\hline - 2000 & -0.244 & 0.173 & -1.411 & 0.159 \\
\hline - 2001 & -0.436 & 0.197 & -2.216 & 0.027 \\
\hline
\end{tabular}


Table 21b: Ordinary Least Squares (OLS) regression model for the impact of index antipsychotic on mental healthcare cost among schizophrenia patients (second stage sample selection models): Risperidone vs. quetiapine (contd.)

\begin{tabular}{lcccc}
\hline \multicolumn{1}{c}{ Beta } & S.E & t-statistic & \multicolumn{2}{c}{$\begin{array}{l}\text { Significance } \\
(\boldsymbol{p})\end{array}$} \\
\hline Pre-index period psychiatric medication use & ( days of supply) \\
Mood stabilizers & 0.011 & 0.000 & 2.204 & 0.028 \\
Antipsychotics & 0.001 & 0.002 & 0.297 & 0.767 \\
Antidepressants & 0.031 & 0.000 & 2.804 & 0.005 \\
Benzodiazepines & -0.090 & 0.149 & -0.605 & 0.546 \\
Index prescription (ref: quetiapine) & & & & \\
Risperidone & 0.084 & 0.448 & 0.187 & 0.852 \\
Inverse Mills Ratio & -0.031 & 0.280 & -0.112 & 0.911 \\
\hline
\end{tabular}

Model fit statistics:

Adjusted R-square $=18.19, \mathbf{F}=4.24, \mathbf{p}=0.00$ 
Table 21c: Ordinary Least Squares (OLS) regression model for the impact of index antipsychotic on mental healthcare cost among schizophrenia patients (second stage sample selection models): Typicals vs. quetiapine

\begin{tabular}{|c|c|c|c|c|}
\hline & Beta & S.E & t-statistic & Significance (p) \\
\hline \multicolumn{5}{|l|}{ Demographic characteristics } \\
\hline Age (in years) & -0.004 & 0.007 & -0.644 & 0.520 \\
\hline Males (ref: females) & 0.002 & 0.001 & 2.461 & 0.014 \\
\hline Whites (ref: non-whites) & 0.199 & 0.205 & 0.974 & 0.331 \\
\hline Metro (ref: non-metro) & -0.299 & 0.176 & -1.695 & 0.091 \\
\hline \multicolumn{5}{|l|}{ Prescribing physician type } \\
\hline Psychiatric prescriber & 0.000 & 0.000 & 1.451 & 0.148 \\
\hline \multicolumn{5}{|c|}{ Schizophrenia subtype (ref: only schizophrenia) } \\
\hline Schizoaffective disorder & 0.019 & 0.208 & 0.093 & 0.926 \\
\hline $\begin{array}{l}\text { Both schizoaffective disorder } \\
\text { and schizophrenia }\end{array}$ & -0.955 & 0.228 & -4.182 & 0.000 \\
\hline \multicolumn{5}{|l|}{ Pre-index co-morbidities } \\
\hline Charlson Comorbidity Index & 0.033 & 0.052 & 0.642 & 0.521 \\
\hline Alcohol and substance abuse & 0.547 & 0.168 & 3.264 & 0.001 \\
\hline Bipolar disorder & -0.004 & 0.232 & -0.017 & 0.986 \\
\hline Major depression & 0.205 & 0.194 & 1.054 & 0.293 \\
\hline Other mental comorbidities & 0.142 & 0.168 & 0.848 & 0.397 \\
\hline \multicolumn{5}{|c|}{ Pre-index healthcare utilization and cost } \\
\hline $\begin{array}{l}\text { Pre-index mental-health } \\
\text { related cost }\end{array}$ & 0.012 & 0.022 & 0.561 & 0.575 \\
\hline $\begin{array}{l}\text { Pre-index mental-health } \\
\text { related ER visits }\end{array}$ & 0.027 & 0.221 & 0.122 & 0.903 \\
\hline $\begin{array}{l}\text { Pre-index mental-health } \\
\text { related hospitalizations }\end{array}$ & 0.434 & 0.174 & 2.495 & 0.013 \\
\hline $\begin{array}{l}\text { Pre-index mental-health } \\
\text { related physician visits }\end{array}$ & -0.017 & 0.051 & -0.332 & 0.740 \\
\hline \multicolumn{5}{|c|}{ Year of index prescription (ref: 1999) } \\
\hline - 2000 & -0.016 & 0.218 & -0.074 & 0.941 \\
\hline - 2001 & -0.129 & 0.282 & -0.459 & 0.646 \\
\hline
\end{tabular}


Table 21c: Ordinary Least Squares (OLS) regression model for the impact of index antipsychotic on mental healthcare cost among schizophrenia patients (second stage sample selection models): Typicals vs. quetiapine (contd.)

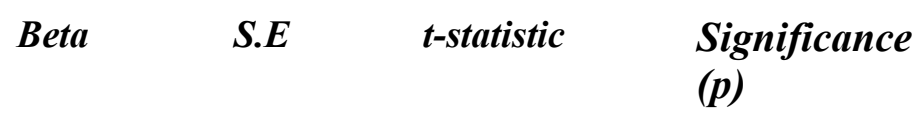

\begin{tabular}{lllll}
\hline \multicolumn{3}{l}{ Pre-index period psychiatric medication use ( days of supply) } & \\
Mood stabilizers & 0.045 & 0.001 & 1.789 & 0.074 \\
Antipsychotics & 0.002 & 0.002 & 1.125 & 0.261 \\
Antidepressants & 0.021 & 0.000 & 2.866 & 0.004 \\
Benzodiazepines & 0.127 & 0.169 & 0.754 & 0.452 \\
Index prescription (ref: quetiapine) & & & & \\
Typical & 0.001 & 0.001 & 1.450 & 0.148 \\
Inverse Mills Ratio & 0.394 & 0.354 & 1.114 & 0.266 \\
\hline
\end{tabular}

\section{Model fit statistics:}

Adjusted R-square $=20.0 \%, \mathbf{F}=5.71, \mathbf{p}=0.00$ 
Results for the OLS regression models for the impact of index antipsychotics on total and mental healthcare costs are presented in the tables 20a-20c for total and tables 21a-21c for mental healthcare costs, respectively. The results showed that there were no significant differences in total and mental healthcare-related costs between quetiapine and other index antipsychotics. The model was further tested for appropriate specifications. It was possible that multicollinarity resulting due to inclusion of IMR in the model may be inflating the standard errors. Therefore, a semi-log OLS model was developed without including the IMR and the output was compared with the outputs of regression models that included IMR. As the direction and significance of variables in all these outputs were similar, it was concluded that there were no significant differences in the total and mental healthcare costs between these cohorts.

\section{Bipolar disorder}

Results for univariate analysis comparing differences in total and mental costs for bipolar disorder patients are reported in table 22. Total post-index costs for quetiapine $(\$ 13,227 \pm \$ 18,862)$ were significantly lesser compared to risperidone $(\$ 17,539 \pm$ $\$ 17,570)$ and typical antipsychotics $(\$ 17,570 \pm \$ 23,842)$. Total post-index mental health-related costs for quetiapine $(\$ 8,064 \pm \$ 7,368)$ were significantly lesser than for olanzapine $(\$ 10,203 \pm \$ 17,203)$.

The results of the first-stage multinomial logistic regression model are presented in table 23. Results of multivariate analysis show that there were no significant differences in total healthcare costs of the quetiapine cohort as compared to other index atypical antipsychotic cohorts. However, typical antipsychotics had $28.4 \%$ greater total 
cost and $8.7 \%$ greater mental health-related costs than quetiapine. Results for the OLS regression models for the impact of index antipsychotics on total and mental healthcare costs are presented in tables $24 a-24 c$ for total and table $25 a-25 c$ for mental health-related costs, respectively.

\section{Results for research objective 9}

For schizophrenia and bipolar disorder patients, to compare the impact of quetiapine with risperidone, olanzapine, and typical antipsychotics on components of mental healthcare costs (costs associated with mental health-related inpatient, emergency room, outpatient and pharmacy services)

$\underline{\text { Schizophrenia }}$

Univariate analysis for mental health-related inpatient, outpatient, ER, and pharmacy costs are reported in table18. The results indicated that the post-index inpatient costs were significantly higher for typical antipsychotics $(\$ 5,418 \pm \$ 15,787)$ compared to quetiapine $(\$ 4,865+\$ 11,285)$. The post-index outpatient costs were also significantly higher for risperidone $(\$ 4,247 \pm \$ 10,209)$ and olanzapine $(\$ 4,136 \pm \$ 16,349)$ as compared to quetiapine $(\$ 2,071 \pm \$ 3,479)$. The post-index ER costs were significantly lower for risperidone $(\$ 152 \pm \$ 415)$ as compared to quetiapine $(\$ 231 \pm \$ 531)$. Compared to quetiapine $(\$ 2470 \pm 1789)$, the post-index prescription costs were significantly higher for olanzapine $(\$ 3,072 \pm 2,285)$ and significantly lower for typical antipsychotics $(\$ 1,421 \pm \$ 1,898)$. 
Table 22: Healthcare costs comparison among bipolar disorder patients: (Quetiapine versus other antipsychotics)

\begin{tabular}{|c|c|c|c|c|}
\hline & $\begin{array}{c}\text { Olanzapine } \\
N=283\end{array}$ & $\begin{array}{c}\text { Risperidone } \\
\quad N=201\end{array}$ & $\begin{array}{c}\text { Quetiapine } \\
N=106\end{array}$ & $\begin{array}{l}\text { Typical } \\
N=205\end{array}$ \\
\hline \multicolumn{5}{|c|}{ Total Healthcare Costs } \\
\hline \multicolumn{5}{|c|}{$\begin{array}{l}\text { Total } \\
\text { Mean }( \pm \text { std })\end{array}$} \\
\hline Pre-index & $\begin{array}{c}\$ 10,382 \\
(+\$ 16,609)\end{array}$ & $\begin{array}{c}\$ 13,037^{Q} \\
( \pm \$ 28,248)\end{array}$ & $\begin{array}{c}\$ 9,993 \\
(+\$ 11,103)\end{array}$ & $\begin{array}{c}\$ 9,533 \\
(+\$ 11,582)\end{array}$ \\
\hline Post-index & $\begin{array}{c}\$ 15,866 \\
(+\$ 23,164)\end{array}$ & $\begin{array}{l}\$ 17,539^{Q} \\
( \pm \$ 28,672)\end{array}$ & $\begin{array}{l}\$ 13,227^{\text {ORT }} \\
( \pm \$ 18,862)\end{array}$ & $\begin{array}{l}\$ 17,570^{Q} \\
( \pm \$ 23,842)\end{array}$ \\
\hline \multicolumn{5}{|c|}{ Mental health-related Costs } \\
\hline \multicolumn{5}{|c|}{$\begin{array}{l}\text { Total mental health-related } \\
\text { Mean }( \pm \text { std })\end{array}$} \\
\hline Pre-index & $\begin{array}{c}\$ 6,286 \\
( \pm \$ 8,918)\end{array}$ & $\begin{array}{c}\$ 7,604^{Q} \\
(+\$ 20,688)\end{array}$ & $\begin{array}{l}\$ 6,377^{\mathrm{RT}} \\
( \pm \$ 9,136)\end{array}$ & $\begin{array}{c}\$ 4,223^{Q} \\
( \pm \$ 6,911)\end{array}$ \\
\hline Post-index & $\begin{array}{l}\$ 10,203^{Q} \\
( \pm \$ 17,724)\end{array}$ & $\begin{array}{c}\$ 9,475 \\
( \pm \$ 14,202)\end{array}$ & $\begin{array}{c}\$ 8,0640 \\
( \pm \$ 11,615)\end{array}$ & $\begin{array}{c}\$ 7,368 \\
( \pm \$ 11,239)\end{array}$ \\
\hline \multicolumn{5}{|l|}{$\begin{array}{l}\text { Inpatient } \\
\text { Mean }( \pm \text { std })\end{array}$} \\
\hline Pre-index & $\begin{array}{c}\$ 3,785 \\
(+\$ 7,219)\end{array}$ & $\begin{array}{c}\$ 4,107 \\
(+\$ 19,069)\end{array}$ & $\begin{array}{c}\$ 3,873 \\
( \pm \$ 7,161)\end{array}$ & $\begin{array}{c}\$ 2,274 \\
(+\$ 5,416)\end{array}$ \\
\hline Post-index & $\begin{array}{c}\$ 3,753 \\
( \pm \$ 8,956)\end{array}$ & $\begin{array}{c}\$ 3,633 \\
( \pm \$ 9,592)\end{array}$ & $\begin{array}{c}\$ 4,054 \\
( \pm \$ 10,345)\end{array}$ & $\begin{array}{c}\$ 3,968 \\
( \pm \$ 9,305)\end{array}$ \\
\hline \multicolumn{5}{|c|}{$\begin{array}{l}\text { Outpatient } \\
\text { Mean }( \pm \text { std })\end{array}$} \\
\hline Pre-index & $\begin{array}{c}\$ 2,017 \\
( \pm \$ 4,295)\end{array}$ & $\begin{array}{c}\$ 3,045 \\
(+\$ 7,731)\end{array}$ & $\begin{array}{c}\$ 2,042 \\
( \pm \$ 4,753)\end{array}$ & $\begin{array}{c}\$ 1,436 \\
( \pm \$ 3,096)\end{array}$ \\
\hline Post-index & $\begin{array}{c}\$ 3,580 \\
(+\$ 14,553)\end{array}$ & $\begin{array}{c}\$ 3,610 \\
(+\$ 8,678)\end{array}$ & $\begin{array}{c}\$ 2,945 \\
( \pm \$ 4,456)\end{array}$ & $\begin{array}{c}\$ 2,124 \\
( \pm \$ 4,443)\end{array}$ \\
\hline \multicolumn{5}{|c|}{$\begin{array}{l}\text { ER } \\
\text { Mean }(+ \text { std })\end{array}$} \\
\hline Pre-index & $\begin{array}{c}\$ 127 \\
( \pm \$ 435)\end{array}$ & $\begin{array}{c}\$ 97 \\
(+\$ 339)\end{array}$ & $\begin{array}{c}\$ 89^{\mathrm{T}} \\
( \pm \$ 214)\end{array}$ & $\begin{array}{c}\$ 43^{Q} \\
( \pm \$ 199)\end{array}$ \\
\hline Post-index & $\begin{array}{c}\$ 218 \\
(+\$ 1,223)\end{array}$ & $\begin{array}{c}\$ 129^{Q} \\
(+\$ 352)\end{array}$ & $\begin{array}{c}\$ 200^{\mathrm{R}} \\
( \pm \$ 486)\end{array}$ & $\begin{array}{c}\$ 157 \\
(+\$ 402)\end{array}$ \\
\hline
\end{tabular}


Table 22: Healthcare costs comparison among bipolar disorder patients: (Quetiapine versus other antipsychotics) (contd.)

\begin{tabular}{lcccc}
\hline & $\begin{array}{c}\text { Olanzapine } \\
\mathbf{N}=\mathbf{2 8 3}\end{array}$ & $\begin{array}{c}\text { Risperidone } \\
\mathbf{N}=\mathbf{2 0 1}\end{array}$ & $\begin{array}{c}\text { Quetiapine } \\
\boldsymbol{N}=\mathbf{1 0 6}\end{array}$ & $\begin{array}{c}\text { Typical } \\
\mathbf{N}=\mathbf{2 0 5}\end{array}$ \\
\hline $\begin{array}{l}\text { Prescription } \\
\text { Mean }( \pm \text { std) }\end{array}$ & & & & \\
Pre-index & $\$ 356$ & $\$ 354$ & $\$ 372$ & $\$ 469$ \\
& $( \pm \$ 578)$ & $( \pm \$ 594)$ & $( \pm \$ 503)$ & $( \pm \$ 759)$ \\
Post-index & $\$ 2,650$ & $\$ 2,102$ & $\$ 2,084^{\mathrm{T}}$ & $\$ 1,118 \mathrm{Q}$ \\
& $( \pm \$ 1,970)$ & $( \pm \$ 1,761)$ & $( \pm \$ 1,578)$ & $( \pm \$ 1,410)$ \\
\hline
\end{tabular}

${ }^{\mathrm{O}}$ Significant difference between quetiapine and olanzapine cohorts at $\mathrm{p}<0.05$

${ }^{\mathrm{R}}$ Significant difference between quetiapine and risperidone cohorts at $\mathrm{p}<0.05$

${ }^{\mathrm{T}}$ Significant difference between quetiapine and typicals cohorts at $\mathrm{p}<0.05$ 
Table 23: Multinomial logistic regression model determining predictors of index antipsychotic use among bipolar disorder patients (first-stage sample selection model)

\begin{tabular}{|c|c|c|c|c|c|c|}
\hline & \multicolumn{2}{|c|}{$\begin{array}{c}\text { Olanzapine vs. } \\
\text { Quetiapine }\end{array}$} & \multicolumn{2}{|c|}{$\begin{array}{c}\text { Risperidone vs. } \\
\text { Quetiapine }\end{array}$} & \multicolumn{2}{|c|}{$\begin{array}{l}\text { Typicals vs. } \\
\text { Quetiapine }\end{array}$} \\
\hline & Coefficient & t-ratio & Coefficient & t-ratio & Coefficient & t-ratio \\
\hline \multicolumn{7}{|c|}{ Demographic characteristics } \\
\hline Age (in years) & -0.001 & -0.074 & 0.001 & 0.101 & 0.029 & 2.329 \\
\hline Age category & -0.687 & -1.688 & -0.979 & -2.355 & -1.352 & -3.211 \\
\hline Males (ref: females) & 0.070 & 0.419 & 0.002 & 1.002 & 0.001 & 0.431 \\
\hline $\begin{array}{l}\text { Whites (ref: non- } \\
\text { whites) }\end{array}$ & 0.435 & 0.964 & 0.051 & 0.113 & -0.167 & -0.360 \\
\hline $\begin{array}{l}\text { Metro (ref: non- } \\
\text { metro) }\end{array}$ & 0.304 & 0.774 & -0.236 & -0.594 & 0.148 & 0.351 \\
\hline \multicolumn{7}{|c|}{ Prescribing physician type } \\
\hline Psychiatric prescriber & -0.012 & $-3.223^{*}$ & -0.011 & $-3.408^{*}$ & -0.011 & $-3.372^{*}$ \\
\hline \multicolumn{7}{|c|}{ Bipolar disorder subtype (ref: only bipolar disorder) } \\
\hline Bipolar Disorder II & -0.976 & $-2.331^{*}$ & -0.851 & $-2.032^{*}$ & -1.457 & $-3.212^{*}$ \\
\hline $\begin{array}{l}\text { Bipolar Disorder } \\
\text { Mixed }\end{array}$ & -1.150 & $-2.788^{*}$ & -2.132 & $-5.038^{*}$ & -1.426 & $-3.265^{*}$ \\
\hline \multicolumn{7}{|c|}{ Pre-index co-morbidities } \\
\hline Diabetes & 0.509 & 1.082 & 0.706 & 1.455 & 0.974 & $1.993^{*}$ \\
\hline Hyperlipidemia & -0.316 & -0.639 & -0.193 & -0.373 & 0.318 & 0.621 \\
\hline Hypertension & -0.266 & -0.792 & 0.181 & 0.510 & 0.102 & 0.276 \\
\hline $\begin{array}{l}\text { Alcohol and substance } \\
\text { abuse }\end{array}$ & -0.435 & -1.454 & -0.870 & $-2.634^{*}$ & -0.198 & -0.596 \\
\hline Bipolar disorder & 0.112 & 0.307 & -0.207 & -0.527 & -0.050 & -0.119 \\
\hline Major depression & 0.241 & 0.742 & -0.110 & -0.313 & -0.186 & -0.485 \\
\hline Anxiety disorder & 0.026 & 0.073 & -0.203 & -0.532 & -0.149 & -0.369 \\
\hline Personality disorder & -0.042 & -0.104 & -0.081 & -0.190 & -0.300 & -0.656 \\
\hline $\begin{array}{l}\text { Non-specified } \\
\text { psychosis }\end{array}$ & 0.661 & 0.810 & 0.840 & 0.985 & 0.477 & 0.531 \\
\hline $\begin{array}{l}\text { Other affective } \\
\text { psychosis }\end{array}$ & -0.356 & -0.436 & 0.313 & 0.379 & -28.865 & 0.000 \\
\hline
\end{tabular}


Table 23: Multinomial logistic regression model determining predictors of index antipsychotic use among bipolar disorder patients (first-stage sample selection model) (contd.)

\begin{tabular}{|c|c|c|c|c|c|c|}
\hline & \multicolumn{2}{|c|}{$\begin{array}{c}\text { Olanzapine vs. } \\
\text { Quetiapine }\end{array}$} & \multicolumn{2}{|c|}{$\begin{array}{c}\text { Risperidone vs. } \\
\text { Quetiapine }\end{array}$} & \multicolumn{2}{|c|}{$\begin{array}{l}\text { Typicals vs. } \\
\text { Quetiapine }\end{array}$} \\
\hline & Coefficient & t-ratio & Coefficient & t-ratio & Coefficient & t-ratio \\
\hline $\begin{array}{l}\text { Attention deficit } \\
\text { disorders }\end{array}$ & -0.438 & -0.554 & 1.196 & 1.624 & 0.727 & 0.862 \\
\hline $\begin{array}{l}\text { Mild to moderate } \\
\text { depression }\end{array}$ & 0.091 & 0.236 & 0.311 & 0.765 & -0.382 & -0.814 \\
\hline \multicolumn{7}{|c|}{ Pre-index healthcare utilization and cost } \\
\hline $\begin{array}{l}\text { Number of pre-index } \\
\text { mental-health related } \\
\text { ER visits }\end{array}$ & 0.000 & 0.936 & 0.000 & 0.327 & 0.000 & -0.631 \\
\hline $\begin{array}{l}\text { Number of pre-index } \\
\text { mental-health related } \\
\text { hospitalizations }\end{array}$ & 0.003 & 0.023 & -0.069 & -0.543 & -0.254 & -1.593 \\
\hline $\begin{array}{l}\text { Number of } \\
\text { psychotherapy visits }\end{array}$ & 0.002 & 0.070 & -0.037 & -1.015 & 0.012 & 0.408 \\
\hline $\begin{array}{l}\text { Number of medication } \\
\text { management visits }\end{array}$ & -0.087 & $-2.383^{*}$ & -0.058 & -1.464 & -0.158 & $-3.350^{*}$ \\
\hline \multicolumn{7}{|c|}{ Year of index prescription (ref: 1999) } \\
\hline - 2000 & -0.119 & -0.363 & 0.220 & 0.633 & -0.396 & -1.109 \\
\hline - 2001 & -0.292 & -0.844 & -0.120 & -0.320 & -1.256 & $-3.032^{*}$ \\
\hline \multicolumn{7}{|c|}{ Pre-index period psychiatric medication use ( days of supply) } \\
\hline Mood stabilizers & 0.001 & 0.652 & 0.000 & -0.129 & -0.002 & -1.948 \\
\hline Anticholinergics & -0.006 & -1.291 & -0.020 & -1.560 & -0.007 & -0.971 \\
\hline $\begin{array}{l}\text { Anxiolytics/hypnotics/ } \\
\text { sedatives }\end{array}$ & 0.897 & 0.052 & 0.000 & -0.058 & 0.001 & 0.354 \\
\hline Antipsychotics & -0.004 & -0.707 & 0.011 & $2.005^{*}$ & 0.012 & $2.119^{*}$ \\
\hline Antidepressants & 0.001 & 0.866 & 0.000 & 0.197 & -0.316 & -0.035 \\
\hline Benzodiazepines & -0.012 & $-2.178^{*}$ & -0.002 & -1.816 & -0.001 & -1.341 \\
\hline Antiparkinsons & -0.002 & -0.587 & -0.006 & -1.406 & -0.001 & -0.389 \\
\hline
\end{tabular}

*significant at $\mathrm{p}<0.05$ level

Model fit statistics:

Pseudo R-square $=12.94 \%,-2$ Log Likelihood $=957.41 ; \chi^{2}=284.75 ; p=0.00$ 
Table 24a: Ordinary Least Squares (OLS) regression model for the impact of index antipsychotic on total healthcare cost among bipolar disorder patients (second stage sample selection models): Olanzapine vs. quetiapine

\begin{tabular}{|c|c|c|c|c|}
\hline & Beta & S.E & t-statistic & Significance (p) \\
\hline \multicolumn{5}{|l|}{ Demographic characteristics } \\
\hline Age (in years) & 0.006 & 0.004 & 1.432 & 0.153 \\
\hline Agecat & 0.063 & 0.144 & 0.438 & 0.662 \\
\hline Males (ref: females) & 0.502 & 0.001 & 0.049 & 0.961 \\
\hline Whites (ref: non-whites) & 0.199 & 0.176 & 1.129 & 0.260 \\
\hline Metro (ref: non-metro) & -0.028 & 0.127 & -0.217 & 0.828 \\
\hline \multicolumn{5}{|l|}{ Prescribing physician type } \\
\hline Psychiatric prescriber & 0.012 & 0.000 & 1.972 & 0.049 \\
\hline \multicolumn{5}{|c|}{ Bipolar disorder subtype (ref: bipolar disorder I) } \\
\hline Bipolar disorder II disorder & -0.163 & 0.141 & -1.154 & 0.249 \\
\hline Mixed & -0.189 & 0.174 & -1.085 & 0.279 \\
\hline \multicolumn{5}{|l|}{ Pre-index co-morbidities } \\
\hline Charlson Comorbidity Index & 0.135 & 0.049 & 2.758 & 0.006 \\
\hline Alcohol and substance abuse & 0.226 & 0.115 & 1.959 & 0.051 \\
\hline Bipolar disorder & 0.100 & 0.147 & 0.681 & 0.496 \\
\hline Major depression & 0.011 & 0.117 & 0.096 & 0.923 \\
\hline Other mental comorbidities & 0.049 & 0.109 & 0.454 & 0.650 \\
\hline \multicolumn{5}{|c|}{ Pre-index healthcare utilization and cost } \\
\hline $\begin{array}{l}\text { Pre-index total health related } \\
\text { cost }\end{array}$ & 0.229 & 0.042 & 5.422 & 0.000 \\
\hline $\begin{array}{l}\text { Pre-index total health related } \\
\text { ER visits }\end{array}$ & -0.254 & 0.138 & -1.842 & 0.066 \\
\hline $\begin{array}{l}\text { Pre-index total health related } \\
\text { hospitalizations }\end{array}$ & -0.097 & 0.120 & -0.806 & 0.421 \\
\hline $\begin{array}{l}\text { Pre-index total health related } \\
\text { physician visits }\end{array}$ & 0.008 & 0.006 & 1.266 & 0.206 \\
\hline \multicolumn{5}{|c|}{ Year of index prescription (ref: 1999) } \\
\hline - 2000 & -0.006 & 0.124 & -0.044 & 0.965 \\
\hline - 2001 & -0.235 & 0.146 & -1.611 & 0.108 \\
\hline
\end{tabular}


Table 24a: Ordinary Least Squares (OLS) regression model for the impact of index antipsychotic on total healthcare cost among bipolar disorder patients (second stage sample selection models): Olanzapine vs. quetiapine (contd.)

\begin{tabular}{lcccc}
\hline & \multicolumn{1}{c}{ Beta } & S.E & t-statistic & Significance (p) \\
\hline \multicolumn{2}{l}{ Pre-index period psychiatric medication } & use & ( days of supply) & \\
Mood stabilizers & 0.041 & 0.000 & 2.317 & 0.021 \\
Antipsychotics & 0.003 & 0.002 & 1.581 & 0.115 \\
Antidepressants & 0.000 & 0.000 & 0.654 & 0.513 \\
Benzodiazepines & 0.050 & 0.114 & 0.437 & 0.663 \\
Index prescription (ref: quetiapine) & & & \\
Olanzapine & 0.261 & 0.495 & 0.527 & 0.059 \\
Inverse Mills Ratio & -0.058 & 0.295 & -0.197 & 0.844 \\
\hline
\end{tabular}

\section{Model fit statistics:}

Adjusted R-square $=22.22 \%, F=5.77, p=0.000$ 
Table 24b: Ordinary Least Squares (OLS) regression model for the impact of index antipsychotic on total healthcare cost among bipolar disorder patients (second stage sample selection models): Risperidone vs. quetiapine

\begin{tabular}{|c|c|c|c|c|}
\hline & Beta & S.E & t-statistic & Significance (p) \\
\hline \multicolumn{5}{|l|}{ Demographic characteristics } \\
\hline Age (in years) & 0.012 & 0.005 & 2.382 & 0.018 \\
\hline agecat & 0.115 & 0.173 & 0.661 & 0.509 \\
\hline Males (ref: females) & 0.000 & 0.001 & 0.368 & 0.714 \\
\hline Whites (ref: non-whites) & 0.481 & 0.206 & 2.34 & 0.020 \\
\hline Metro (ref: non-metro) & -0.266 & 0.160 & -1.657 & 0.099 \\
\hline \multicolumn{5}{|l|}{ Prescribing physician type } \\
\hline Psychiatric prescriber & 0.506 & 0.000 & 0.293 & 0.770 \\
\hline \multicolumn{5}{|c|}{ Bipolar disorder subtype (ref: Bipolar disorder I) } \\
\hline Bipolar disorder II & -0.001 & 0.174 & -0.005 & 0.996 \\
\hline Mixed & -0.509 & 0.234 & -2.174 & 0.031 \\
\hline \multicolumn{5}{|l|}{ Pre-index co-morbidities } \\
\hline Charlson Comorbidity Index & 0.178 & 0.055 & 3.266 & 0.001 \\
\hline Alcohol and substance abuse & 0.235 & 0.184 & 1.277 & 0.203 \\
\hline Bipolar disorder & 0.194 & 0.193 & 1.006 & 0.315 \\
\hline Major depression & 0.019 & 0.165 & 0.114 & 0.909 \\
\hline Other mental comorbidities & -0.019 & 0.141 & -0.135 & 0.892 \\
\hline \multicolumn{5}{|c|}{ Pre-index healthcare utilization and cost } \\
\hline $\begin{array}{l}\text { Pre-index total health related } \\
\text { cost }\end{array}$ & 0.089 & 0.022 & 4.007 & 0.000 \\
\hline $\begin{array}{l}\text { Pre-index total health related } \\
\text { ER visits }\end{array}$ & 0.230 & 0.182 & 1.265 & 0.207 \\
\hline $\begin{array}{l}\text { Pre-index total health related } \\
\text { hospitalizations }\end{array}$ & -0.134 & 0.141 & -0.95 & 0.343 \\
\hline $\begin{array}{l}\text { Pre-index total health related } \\
\text { physician visits }\end{array}$ & 0.010 & 0.010 & 0.993 & 0.322 \\
\hline \multicolumn{5}{|c|}{ Year of index prescription (ref: 1999) } \\
\hline - 2000 & -0.119 & 0.168 & -0.711 & 0.478 \\
\hline - 2001 & -0.522 & 0.193 & -2.698 & 0.007 \\
\hline
\end{tabular}


Table 24b: Ordinary Least Squares (OLS) regression model for the impact of index antipsychotic on total healthcare cost among bipolar disorder patients (second stage sample selection models): Risperidone vs. quetiapine (contd.)

\begin{tabular}{lcccc}
\hline & \multicolumn{1}{c}{ Beta } & S.E & t-statistic & Significance (p) \\
\hline \multicolumn{2}{l}{ Pre-index period psychiatric medication } & use & (days of supply) & \\
Mood stabilizers & 0.031 & 0.000 & 2.877 & 0.004 \\
Antipsychotics & -0.001 & 0.001 & -0.368 & 0.713 \\
Antidepressants & 0.507 & 0.387 & 1.309 & 0.192 \\
Benzodiazepines & 0.032 & 0.141 & 0.226 & 0.822 \\
Index prescription (ref: quetiapine) & & & \\
Risperidone & -0.329 & 0.439 & -0.751 & 0.454 \\
Inverse Mills Ratio & 0.314 & 0.268 & 1.171 & 0.243 \\
\hline
\end{tabular}

\section{Model fit statistics:}

Adjusted R-square $=27.58 \%, F=5.66, p=0.000$ 
Table 24c: Ordinary Least Squares (OLS) regression model for the impact of index antipsychotic on total healthcare cost among bipolar disorder patients (second stage sample selection models): Typicals vs. quetiapine

\begin{tabular}{|c|c|c|c|c|}
\hline & Beta & S.E & t-statistic & Significance (p) \\
\hline \multicolumn{5}{|l|}{ Demographic characteristics } \\
\hline Age (in years) & 0.002 & 0.006 & 0.348 & 0.728 \\
\hline agecat & 0.278 & 0.179 & 1.553 & 0.122 \\
\hline Males (ref: females) & 0.000 & 0.001 & 0.324 & 0.746 \\
\hline Whites (ref: non-whites) & 0.431 & 0.211 & 2.04 & 0.042 \\
\hline Metro (ref: non-metro) & -0.280 & 0.193 & -1.451 & 0.148 \\
\hline \multicolumn{5}{|l|}{ Prescribing physician type } \\
\hline Psychiatric prescriber & 0.000 & 0.000 & 1.384 & 0.168 \\
\hline \multicolumn{5}{|c|}{ Bipolar disorder subtype (ref: Bipolar disorder I ) } \\
\hline Bipolar disorder II & 0.389 & 0.221 & 1.758 & 0.080 \\
\hline Mixed & 0.020 & 0.213 & 0.095 & 0.924 \\
\hline \multicolumn{5}{|l|}{ Pre-index co-morbidities } \\
\hline Charlson Comorbidity Index & 0.212 & 0.049 & 4.306 & 0.000 \\
\hline Alcohol and substance abuse & 0.215 & 0.175 & 1.23 & 0.220 \\
\hline Bipolar disorder & -0.272 & 0.236 & -1.153 & 0.250 \\
\hline Major depression & 0.176 & 0.197 & 0.896 & 0.371 \\
\hline Other mental comorbidities & 0.360 & 0.162 & 2.23 & 0.027 \\
\hline \multicolumn{5}{|c|}{ Pre-index healthcare utilization and cost } \\
\hline $\begin{array}{l}\text { Pre-index total health related } \\
\text { cost }\end{array}$ & 0.244 & 0.066 & 3.727 & 0.000 \\
\hline $\begin{array}{l}\text { Pre-index total health related } \\
\text { ER visits }\end{array}$ & 0.304 & 0.221 & 1.378 & 0.169 \\
\hline $\begin{array}{l}\text { Pre-index total health related } \\
\text { hospitalizations }\end{array}$ & 0.185 & 0.191 & 0.97 & 0.333 \\
\hline $\begin{array}{l}\text { Pre-index total health related } \\
\text { physician visits }\end{array}$ & 0.004 & 0.011 & 0.353 & 0.725 \\
\hline \multicolumn{5}{|c|}{ Year of index prescription (ref: 1999) } \\
\hline - 2000 & -0.053 & 0.201 & -0.262 & 0.794 \\
\hline - 2001 & -0.382 & 0.258 & -1.479 & 0.140 \\
\hline
\end{tabular}


Table 24c: Ordinary Least Squares (OLS) regression model for the impact of index antipsychotic on total healthcare cost among bipolar disorder patients (second stage sample selection models): Typicals vs. quetiapine (contd.)

\begin{tabular}{lcccc}
\hline & Beta & S.E & t-statistic & Significance (p) \\
\hline Pre-index period psychiatric medication use & (days of supply) & \\
Mood stabilizers & 0.001 & 0.001 & 1.015 & 0.311 \\
Antipsychotics & -0.001 & 0.002 & -0.548 & 0.584 \\
Antidepressants & 0.001 & 0.000 & 1.032 & 0.303 \\
Benzodiazepines & 0.045 & 0.157 & 0.285 & 0.776 \\
Index prescription (ref: quetiapine) & & & \\
Typical & 0.250 & 0.417 & 2.30 & 0.028 \\
Inverse Mills Ratio & 0.083 & 0.260 & 0.319 & 0.750 \\
\hline
\end{tabular}

\section{Model Statistics:}

Adjusted R-square $=31.72 \%, \mathbf{F}=6.76, \mathbf{p}=0.000$ 
Table 25a: Ordinary Least Squares (OLS) regression model for the impact of index antipsychotic on mental healthcare cost among bipolar disorder patients (second stage sample selection models): Olanzapine vs. quetiapine

\begin{tabular}{|c|c|c|c|c|}
\hline & Beta & S.E & t-statistic & Significance (p) \\
\hline \multicolumn{5}{|l|}{ Demographic characteristics } \\
\hline Age (in years) & 0.004 & 0.005 & 0.829 & 0.407 \\
\hline Agecat & 0.104 & 0.157 & 0.665 & 0.506 \\
\hline Males (ref: females) & 0.002 & 0.001 & 1.581 & 0.115 \\
\hline Whites (ref: non-whites) & 0.293 & 0.192 & 1.525 & 0.128 \\
\hline Metro (ref: non-metro) & -0.122 & 0.139 & -0.878 & 0.380 \\
\hline \multicolumn{5}{|l|}{ Prescribing physician type } \\
\hline Psychiatric prescriber & 0.000 & 0.000 & 1.831 & 0.068 \\
\hline \multicolumn{5}{|c|}{ Bipolar disorder subtype (ref: Bipolar disorder I) } \\
\hline Bipolar disorder II & -0.219 & 0.154 & -1.420 & 0.156 \\
\hline Mixed & -0.444 & 0.189 & -2.346 & 0.020 \\
\hline \multicolumn{5}{|l|}{ Pre-index co-morbidities } \\
\hline Charlson Comorbidity Index & 0.117 & 0.051 & 2.285 & 0.023 \\
\hline Alcohol and substance abuse & 0.363 & 0.126 & 2.890 & 0.004 \\
\hline Schizophrenia & 0.006 & 0.160 & 0.036 & 0.971 \\
\hline Major depression & 0.067 & 0.128 & 0.519 & 0.604 \\
\hline Other mental comorbidities & 0.114 & 0.119 & 0.956 & 0.340 \\
\hline \multicolumn{5}{|c|}{ Pre-index healthcare utilization and cost } \\
\hline $\begin{array}{l}\text { Pre-index mental-health } \\
\text { related cost }\end{array}$ & 0.074 & 0.017 & 4.366 & 0.000 \\
\hline $\begin{array}{l}\text { Pre-index mental-health } \\
\text { related ER visits }\end{array}$ & -0.302 & 0.146 & -2.064 & 0.040 \\
\hline $\begin{array}{l}\text { Pre-index mental-health } \\
\text { related hospitalizations }\end{array}$ & 0.158 & 0.122 & 1.296 & 0.196 \\
\hline $\begin{array}{l}\text { Pre-index mental-health } \\
\text { related physician visits }\end{array}$ & 0.001 & 0.031 & 0.024 & 0.981 \\
\hline \multicolumn{5}{|c|}{ Year of index prescription (ref: 1999) } \\
\hline - 2000 & 0.019 & 0.136 & 0.139 & 0.890 \\
\hline - 2001 & -0.143 & 0.157 & -0.907 & 0.365 \\
\hline
\end{tabular}


Table 25a: Ordinary Least Squares (OLS) regression model for the impact of index antipsychotic on mental healthcare cost among bipolar disorder patients (second stage sample selection models): Olanzapine vs. quetiapine (contd.)

\begin{tabular}{lcccc}
\hline & Beta & S.E & t-statistic & Significance (p) \\
\hline \multicolumn{2}{l}{ Pre-index period psychiatric medication } & use & (days of supply) & \\
Mood stabilizers & 0.001 & 0.000 & 1.676 & 0.095 \\
Antipsychotics & 0.004 & 0.002 & 1.737 & 0.083 \\
Antidepressants & 0.000 & 0.000 & 0.890 & 0.374 \\
Benzodiazepines & 0.020 & 0.124 & 0.160 & 0.873 \\
Index prescription (ref: quetiapine) & & & \\
Olanzapine & 0.203 & 0.539 & 0.377 & 0.070 \\
Inverse Mills Ratio & -0.024 & 0.322 & -0.074 & 0.941 \\
\hline
\end{tabular}

\section{Model fit statistics:}

Adjusted R-square $=20.33 \%, F=5.27, p=0.00$ 
Table 25b: Ordinary Least Squares (OLS) regression model for the impact of index antipsychotic on mental healthcare cost among bipolar disorder patients (second stage sample selection models): Risperidone vs. quetiapine

\begin{tabular}{|c|c|c|c|c|}
\hline & Beta & S.E & t-statistic & Significance (p) \\
\hline \multicolumn{5}{|l|}{ Demographic characteristics } \\
\hline Age (in years) & 0.011 & 0.006 & 1.900 & 0.058 \\
\hline agecat & 0.026 & 0.194 & 0.135 & 0.893 \\
\hline Males (ref: females) & 0.001 & 0.001 & 1.007 & 0.315 \\
\hline Whites (ref: non-whites) & 0.409 & 0.233 & 1.758 & 0.080 \\
\hline Metro (ref: non-metro) & -0.195 & 0.180 & -1.080 & 0.281 \\
\hline \multicolumn{5}{|l|}{ Prescribing physician type } \\
\hline Psychiatric prescriber & 0.535 & 0.000 & 0.276 & 0.783 \\
\hline \multicolumn{5}{|c|}{ Bipolar disorder subtype (ref: Bipolar disorder I) } \\
\hline Bipolar disorder II & -0.227 & 0.196 & -1.155 & 0.249 \\
\hline Mixed & -0.849 & 0.264 & -3.212 & 0.002 \\
\hline \multicolumn{5}{|l|}{ Pre-index co-morbidities } \\
\hline Charlson Comorbidity Index & 0.069 & 0.059 & 1.183 & 0.238 \\
\hline Alcohol and substance abuse & 0.482 & 0.208 & 2.324 & 0.021 \\
\hline Schizophrenia & 0.180 & 0.219 & 0.820 & 0.413 \\
\hline Major depression & 0.088 & 0.186 & 0.471 & 0.638 \\
\hline Other mental comorbidities & 0.046 & 0.161 & 0.285 & 0.776 \\
\hline \multicolumn{5}{|c|}{ Pre-index healthcare utilization and cost } \\
\hline $\begin{array}{l}\text { Pre-index mental-health } \\
\text { related cost }\end{array}$ & 0.038 & 0.018 & 2.057 & 0.041 \\
\hline $\begin{array}{l}\text { Pre-index mental-health } \\
\text { related ER visits }\end{array}$ & 0.193 & 0.195 & 0.990 & 0.323 \\
\hline $\begin{array}{l}\text { Pre-index mental-health } \\
\text { related hospitalizations }\end{array}$ & -0.131 & 0.163 & -0.799 & 0.425 \\
\hline $\begin{array}{l}\text { Pre-index mental-health } \\
\text { related physician visits }\end{array}$ & 0.009 & 0.048 & 0.194 & 0.846 \\
\hline \multicolumn{5}{|c|}{ Year of index prescription (ref: 1999) } \\
\hline - 2000 & -0.179 & 0.187 & -0.953 & 0.341 \\
\hline - 2001 & -0.438 & 0.216 & -2.025 & 0.044 \\
\hline
\end{tabular}


Table 25b: Ordinary Least Squares (OLS) regression model for the impact of index antipsychotic on mental healthcare cost among bipolar disorder patients (second stage sample selection models): Risperidone vs. quetiapine (contd.)

\begin{tabular}{lcccc}
\hline & \multicolumn{1}{c}{ Beta } & S.E & t-statistic & Significance (p) \\
\hline Pre-index period psychiatric medication use & (days of supply) & \\
Mood stabilizers & 0.081 & 0.000 & 1.864 & 0.063 \\
Antipsychotics & 0.001 & 0.002 & 0.315 & 0.753 \\
Antidepressants & 0.051 & 0.000 & 2.759 & 0.006 \\
Benzodiazepines & -0.234 & 0.159 & -1.472 & 0.142 \\
Index prescription (ref: quetiapine) & & & \\
Risperidone & -0.647 & 0.502 & -1.288 & 0.199 \\
Inverse Mills Ratio & 0.405 & 0.307 & 1.322 & 0.187 \\
\hline
\end{tabular}

\section{Model fit statistics:}

Adjusted R-square $=19.56 \%, F=3.98, p=0.000$ 
Table 25c: Ordinary Least Squares (OLS) regression model for the impact of index antipsychotic on mental healthcare cost among bipolar disorder patients (second stage sample selection models): Typicals vs. quetiapine

Beta S.E t-statistic Significance (p)

\begin{tabular}{|c|c|c|c|c|}
\hline \multicolumn{5}{|l|}{ Demographic characteristics } \\
\hline Age (in years) & -0.011 & 0.008 & -1.366 & 0.173 \\
\hline agecat & 0.114 & 0.227 & 0.499 & 0.618 \\
\hline Males (ref: females) & 0.002 & 0.001 & 2.403 & 0.017 \\
\hline Whites (ref: non-whites) & 0.342 & 0.269 & 1.273 & 0.204 \\
\hline Metro (ref: non-metro) & -0.239 & 0.245 & -0.974 & 0.331 \\
\hline \multicolumn{5}{|l|}{ Prescribing physician type } \\
\hline Psychiatric prescriber & 0.000 & 0.000 & 1.738 & 0.083 \\
\hline \multicolumn{5}{|c|}{ Bipolar Disorder subtype (ref: Bipolar disorder I) } \\
\hline Bipolar disorder II & 0.155 & 0.282 & 0.550 & 0.583 \\
\hline Mixed & -0.919 & 0.276 & -3.332 & 0.001 \\
\hline \multicolumn{5}{|l|}{ Pre-index co-morbidities } \\
\hline Charlson Comorbidity Index & 0.072 & 0.057 & 1.275 & 0.203 \\
\hline Alcohol and substance abuse & 0.587 & 0.221 & 2.649 & 0.009 \\
\hline Schizophrenia & -0.117 & 0.301 & -0.388 & 0.699 \\
\hline Major depression & 0.437 & 0.251 & 1.742 & 0.083 \\
\hline Other mental comorbidities & 0.196 & 0.205 & 0.954 & 0.341 \\
\hline \multicolumn{5}{|c|}{ Pre-index healthcare utilization and cost } \\
\hline $\begin{array}{l}\text { Pre-index mental-health } \\
\text { related cost }\end{array}$ & -0.018 & 0.024 & -0.735 & 0.463 \\
\hline $\begin{array}{l}\text { Pre-index mental-health } \\
\text { related ER visits }\end{array}$ & -0.027 & 0.269 & -0.099 & 0.921 \\
\hline $\begin{array}{l}\text { Pre-index mental-health } \\
\text { related hospitalizations }\end{array}$ & 0.592 & 0.218 & 2.719 & 0.007 \\
\hline $\begin{array}{l}\text { Pre-index mental-health } \\
\text { related physician visits }\end{array}$ & 0.024 & 0.062 & 0.387 & 0.699 \\
\hline \multicolumn{3}{|c|}{ Year of index prescription (ref: 1999) } & (contd.) & \\
\hline - 2000 & -0.014 & 0.252 & -0.054 & 0.957 \\
\hline - 2001 & -0.213 & 0.319 & -0.668 & 0.505 \\
\hline
\end{tabular}


Table 25c: Ordinary Least Squares (OLS) regression model for the impact of index antipsychotic on mental healthcare cost among bipolar disorder patients (second stage sample selection models): Typicals vs. quetiapine

\begin{tabular}{lcccc}
\hline & \multicolumn{1}{c}{ Beta } & \multicolumn{1}{c}{$\boldsymbol{S}$. } & t-statistic & Significance $(\boldsymbol{p})$ \\
\hline \multicolumn{2}{l}{ Pre-index period psychiatric medication } & use & (days of supply) & \\
Mood stabilizers & 0.001 & 0.001 & 0.856 & 0.393 \\
Antipsychotics & 0.001 & 0.002 & 0.412 & 0.681 \\
Antidepressants & 0.062 & 0.001 & 3.348 & 0.001 \\
Benzodiazepines & 0.039 & 0.198 & 0.195 & 0.845 \\
Index prescription (ref: quetiapine) & & & \\
Typical & 0.083 & 0.535 & 2.568 & 0.008 \\
Inverse Mills Ratio & 0.357 & 0.332 & 1.076 & 0.283 \\
\hline
\end{tabular}

\section{Model fit statistics:}

Adjusted R-square $=22.41 \%, \mathbf{F}=4.58, \mathbf{p}=0.000$ 


\section{Multivariate analysis results for mental health-related inpatient costs}

The results of the two-part model used to estimate the impact of antipsychotic on mental health-related inpatient costs are presented in the tables $26 \mathrm{a}$ and $26 \mathrm{~b}$. The firstpart logistic regression model comparing the risk of incurring any inpatient cost to no inpatient costs showed no significant difference between quetiapine and other antipsychotics. The second-part semi-log OLS regression which was carried out only on those patients who had incurred any mental health-related inpatient costs showed that patients initiated on typical antipsychotics incurred 3.7\% higher costs than patients initiated on quetiapine.

\section{Multivariate analysis results for mental health-related ER costs}

The results of two part model estimating the impact of index antipsychotic therapy on mental health-related ER costs are presented in table $27 \mathrm{a}$ and $27 \mathrm{~b}$. The first part logistic regression analysis revealed that there were no significant differences in the likelihood of incurring any ER cost between quetiapine and other cohorts. The subsequent semi-log OLS analysis carried out using only the patients who have incurred ER costs also showed no significant differences in mental health-related ER costs between quetiapine and other antipsychotics.

\section{Multivariate analysis results for mental health-related pharmacy costs}

The results show that patients initiated on olanzapine incur $27.88 \%$ higher mental healthcare-related pharmacy cost than patients initiated on quetiapine. Patients initiated on typical antipsychotics incur $53.92 \%$ lesser mental healthcare-related pharmacy cost than patients initiated on quetiapine. Results for the OLS regression models for the 
impact of index antipsychotics on mental-health related pharmacy healthcare costs are presented in table 28 .

\section{Multivariate analysis results for mental health-related outpatient costs}

The results of semi-log regression analysis show that the patients initiated on typical antipsychotics incur 3.0\% higher outpatient costs than patients initiated on quetiapine. Results for the OLS regression models for the impact of index antipsychotics on mental-health related outpatient healthcare costs are presented in table 29.

\section{$\underline{\text { Bipolar Disorder }}$}

Univariate analysis for mental health-related inpatient, outpatient, ER, and pharmacy costs are reported in table 23. There were no significant differences in mentalhealth related inpatient and outpatient costs between quetiapine and other antipsychotics. The post-index ER costs were significantly lower for risperidone $(\$ 129 \pm \$ 352)$ compared to quetiapine $(\$ 200 \pm \$ 486)$. The post-index prescription costs were significantly lower for typicals $(\$ 1,118 \pm \$ 1,118)$ compared to quetiapine $(\$ 2,084 \pm$ $1,578)$.

\section{Multivariate analysis results for mental health-related inpatient costs}

The results of the two-part model used to estimate the impact of antipsychotic on mental health-related inpatient costs are presented in tables 30a and 30b. Results of the two part model show that there were no significant differences in the mental healthrelated inpatient costs between quetiapine and other antipsychotics. Multivariate analysis results for mental health-related ER costs

The results of two part model estimating the impact of index antipsychotic therapy on mental health-related ER costs are presented in tables 31a and 31b. The first 
part logistic regression analysis and the subsequent semi-log OLS analysis revealed that there were no significant differences in the mental health-related ER cost between quetiapine and other cohorts.

Multivariate analysis results for mental health-related pharmacy costs

Patients initiated on typical antipsychotics incur $52.76 \%$ lesser mental healthcarerelated pharmacy cost than patients initiated on quetiapine. Patients initiated on olanzapine incur $12.41 \%$ greater mental healthcare-related pharmacy cost than patients initiated on quetiapine. Results for the OLS regression models for the impact of index antipsychotics on mental-health related pharmacy healthcare costs are presented in table 32.

\section{Multivariate analysis results for mental health-related outpatient costs}

The results of semi-log regression analysis show that the patients initiated patients initiated on typical antipsychotics incur $7.78 \%$ higher outpatient costs than patients initiated on quetiapine. Results for the OLS regression models for the impact of index antipsychotics on mental-health related outpatient healthcare costs are presented in table 33. 
TWO-PART MODEL-PART 1

Table 26a: Logistic regression model for the impact of index antipsychotic on having a mental health-related hospitalization episode among schizophrenia patients

\begin{tabular}{|c|c|c|c|c|c|c|}
\hline & \multirow[t]{2}{*}{ Beta } & \multirow[t]{2}{*}{$S E$} & \multirow[t]{2}{*}{ Sig. (p) } & \multirow[t]{2}{*}{ Odds ratio } & \multicolumn{2}{|c|}{ 95\% CI for odds ratio } \\
\hline & & & & & $\begin{array}{c}\text { Lower } \\
\text { limit }\end{array}$ & $\begin{array}{c}\text { Upper } \\
\text { limit }\end{array}$ \\
\hline \multicolumn{7}{|c|}{ Demographic characteristics } \\
\hline Age (in years) & -0.008 & 0.007 & 0.266 & 0.992 & 0.978 & 1.006 \\
\hline Males (ref: females) & -0.005 & 0.008 & 0.495 & 0.995 & 0.980 & 1.010 \\
\hline $\begin{array}{l}\text { Whites (ref: non- } \\
\text { whites) }\end{array}$ & -0.562 & 0.286 & 0.049 & 0.570 & 0.326 & 0.998 \\
\hline $\begin{array}{l}\text { Metro (ref: non- } \\
\text { metro) }\end{array}$ & 0.361 & 0.195 & 0.065 & 1.435 & 0.979 & 2.105 \\
\hline \multicolumn{7}{|c|}{ Prescribing physician type } \\
\hline $\begin{array}{l}\text { Psychiatric } \\
\text { prescriber }\end{array}$ & 0.000 & 0.000 & 0.730 & 1.000 & 1.000 & 1.000 \\
\hline \multicolumn{7}{|c|}{ Schizophrenia subtype (ref: only schizophrenia) } \\
\hline $\begin{array}{l}\text { Schizoaffective } \\
\text { disorder }\end{array}$ & 0.366 & 0.207 & 0.077 & 1.441 & 0.962 & 2.160 \\
\hline $\begin{array}{l}\text { Both schizoaffective } \\
\text { disorder and } \\
\text { schizophrenia }\end{array}$ & 0.758 & 0.219 & 0.001 & 2.135 & 1.391 & 3.276 \\
\hline \multicolumn{7}{|c|}{ Pre-index co-morbidities } \\
\hline $\begin{array}{l}\text { Alcohol and } \\
\text { substance abuse }\end{array}$ & -0.888 & 0.181 & $<.0001$ & 0.411 & 0.289 & 0.586 \\
\hline Bipolar disorder & -0.362 & 0.244 & 0.138 & 0.697 & 0.432 & 1.123 \\
\hline Major depression & -0.229 & 0.206 & 0.268 & 0.796 & 0.531 & 1.192 \\
\hline $\begin{array}{l}\text { Mild to Moderate } \\
\text { depression }\end{array}$ & -0.246 & 0.236 & 0.297 & 0.782 & 0.492 & 1.242 \\
\hline $\begin{array}{l}\text { Other mental } \\
\text { comorbidities }\end{array}$ & -0.083 & 0.187 & 0.656 & 0.920 & 0.638 & 1.327 \\
\hline diabetes & -0.385 & 0.267 & 0.150 & 0.680 & 0.403 & 1.149 \\
\hline hyperlipedemia & 0.298 & 0.328 & 0.364 & 1.347 & 0.709 & 2.559 \\
\hline hypertension & 0.245 & 0.226 & 0.279 & 1.278 & 0.820 & 1.990 \\
\hline $\begin{array}{l}\text { Charlson } \\
\text { Comorbidity Index }\end{array}$ & -0.197 & 0.063 & 0.002 & 0.821 & 0.726 & 0.930 \\
\hline
\end{tabular}


Table 26a: Logistic regression model for the impact of index antipsychotic on having a mental health-related hospitalization episode among schizophrenia patients(contd.)

\begin{tabular}{|c|c|c|c|c|c|c|}
\hline & \multirow[t]{2}{*}{ Beta } & \multirow[t]{2}{*}{$S E$} & \multirow[t]{2}{*}{ Sig. (p) } & \multirow[t]{2}{*}{ Odds ratio } & \multicolumn{2}{|c|}{ 95\% CI for odds ratio } \\
\hline & & & & & $\begin{array}{c}\text { Lower } \\
\text { limit }\end{array}$ & $\begin{array}{l}\text { Upper } \\
\text { limit }\end{array}$ \\
\hline \multicolumn{7}{|c|}{ Pre-index period psychiatric medication use (days of supply) } \\
\hline Mood stabilizers & 0.000 & 0.001 & 0.722 & 1.000 & 0.999 & 1.001 \\
\hline Antipsychotics & -0.002 & 0.002 & 0.387 & 0.998 & 0.995 & 1.002 \\
\hline Antidepressants & -0.001 & 0.001 & 0.049 & 0.999 & 0.998 & 1.000 \\
\hline Benzodiazepines & -0.001 & 0.001 & 0.345 & 0.999 & 0.998 & 1.001 \\
\hline \multicolumn{7}{|c|}{ Pre-index healthcare utilization and cost } \\
\hline $\begin{array}{l}\text { Number of } \\
\text { psychotherapy visits }\end{array}$ & -0.005 & 0.017 & 0.777 & 0.995 & 0.962 & 1.029 \\
\hline $\begin{array}{l}\text { Number of } \\
\text { medication } \\
\text { management visits }\end{array}$ & 0.076 & 0.030 & 0.011 & 1.079 & 1.018 & 1.143 \\
\hline $\begin{array}{l}\text { Any pre-index } \\
\text { mental-health } \\
\text { related ER visits }\end{array}$ & -0.329 & 0.288 & 0.252 & 0.719 & 0.410 & 1.264 \\
\hline $\begin{array}{l}\text { Pre-index mental- } \\
\text { health related } \\
\text { physician visits }\end{array}$ & 0.039 & 0.055 & 0.484 & 1.039 & 0.933 & 1.158 \\
\hline $\begin{array}{l}\text { Any pre-index } \\
\text { mental-health } \\
\text { related } \\
\text { hospitalizations }\end{array}$ & -0.752 & 0.169 & $<.0001$ & 0.471 & 0.339 & 0.656 \\
\hline \multicolumn{7}{|c|}{ Year of index prescription (ref: 1999) } \\
\hline - 2000 & -0.127 & 0.216 & 0.555 & 0.880 & 0.577 & 1.343 \\
\hline - 2001 & 0.260 & 0.261 & 0.318 & 1.297 & 0.778 & 2.161 \\
\hline \multicolumn{7}{|c|}{ Index prescription (ref: quetiapine) } \\
\hline Risperidone & 0.356 & 0.276 & 0.197 & 1.428 & 0.831 & 2.453 \\
\hline Olanzapine & 0.103 & 0.241 & 0.670 & 1.108 & 0.691 & 1.776 \\
\hline Typicals & 0.202 & 0.260 & 0.436 & 1.224 & 0.736 & 2.037 \\
\hline
\end{tabular}

Model Statistics:

$\mathbf{- 2} \log$ Likehood $=1207.17$, chi-square $=161.00 \quad, p<0.0001$ 
TWO-PART MODEL-PART 2

Table 26b: Ordinary Least Squares (OLS) regression model for the impact of index antipsychotic on mental healthcare-related hospitalization cost among schizophrenia patients

$\begin{array}{cccc}\text { Beta } & \text { S.E } & \text { t-statistic } & \text { Significance } \\ (p)\end{array}$

Demographic characteristics

$\begin{array}{lllll}\text { Age (in years) } & 0.003 & 0.005 & 0.670 & 0.506 \\ \text { Males (ref: females) } & 0.000 & 0.107 & 0.000 & 0.997 \\ \text { Whites (ref: non-whites) } & 0.315 & 0.199 & 1.590 & 0.114 \\ \text { Metro (ref: non-metro) } & 0.114 & 0.131 & 0.870 & 0.384\end{array}$

Prescribing physician type

$\begin{array}{lllll}\text { Psychiatric prescriber } & 0.000 & 0.000 & -0.380 & 0.705\end{array}$

Schizophrenia subtype (ref: only schizophrenia)

$\begin{array}{lllll}\text { Schizoaffective disorder } & -0.084 & 0.129 & -0.650 & 0.515\end{array}$

Both schizoaffective disorder

and schizophrenia

$\begin{array}{llll}-0.122 & 0.206 & -0.590 & 0.554\end{array}$

Pre-index co-morbidities

\begin{tabular}{lcccl} 
Alcohol and substance abuse & 0.301 & 0.107 & 2.800 & 0.006 \\
Bipolar disorder & -0.001 & 0.145 & -0.010 & 0.992 \\
Major depression & 0.153 & 0.126 & 1.210 & 0.226 \\
Mild to Moderate depression & 0.262 & 0.138 & 1.900 & 0.059 \\
Other mental comorbidities & 0.147 & 0.118 & 1.250 & 0.214 \\
diabetes & 0.036 & 0.161 & 0.230 & 0.821 \\
hyperlipedemia & -0.116 & 0.206 & -0.560 & 0.576 \\
hypertension & 0.177 & 0.141 & 1.250 & 0.212 \\
Charlson Comorbidity Index & 0.024 & 0.041 & 0.590 & 0.553 \\
Pre-index period psychiatric medication use`(days of supply) & \\
Mood stabilizers & 0.000 & 0.000 & -1.290 & 0.199 \\
Antipsychotics & 0.001 & 0.001 & 0.880 & 0.378 \\
Antidepressants & 0.000 & 0.000 & 0.090 & 0.929 \\
Benzodiazepines & 0.000 & 0.000 & -1.060 & 0.289 \\
\hline
\end{tabular}


Table 26b: Ordinary Least Squares (OLS) regression model for the impact of index antipsychotic on mental healthcare-related hospitalization cost among schizophrenia patients (contd.)

\begin{tabular}{|c|c|c|}
\hline Beta & S.L & t-statistic \\
\hline
\end{tabular}

Pre-index healthcare utilization and cost

Number of psychotherapy

visits

$\begin{array}{llll}-0.006 & 0.009 & -0.660 & 0.510\end{array}$

Number of medication

management visits

$$
-0.011
$$

0.020

$-0.540$

0.593

Number of pre-index mental-

health related ER visits

$-0.025$

0.073

$-0.340$

0.731

Pre-index mental-health

related physician visits

$-0.060$

0.033

$-1.830$

0.068

Number of pre-index mental-

health related hospitalizations

0.015

0.006

2.590

0.010

Pre-index mental health-

related cost

0.256

0.134

1.910

0.058

Year of index prescription (ref: 1999)

- 2000

$-0.187$

0.166

$-1.120$

0.263

- 2001

$-0.111$

0.175

$-0.640$

0.526

Index prescription (ref: quetiapine)

Risperidone

$-0.269$

0.154

$-1.750$

0.081

Olanzapine

$-0.174$

0.164

$-1.060$

0.290

Typicals

0.036

0.015

0.670

0.026

\section{Model fit statistics:}

Adjusted R-square $=20.07 \%, F=2.14, p<0.0001$ 
TWO-PART MODEL-PART 1

Table 27a: Logistic regression model for the impact of index antipsychotic on having a mental health-related ER episode among schizophrenia patients

\begin{tabular}{|c|c|c|c|c|c|c|}
\hline & \multirow[t]{2}{*}{ Beta } & \multirow[t]{2}{*}{$S E$} & \multirow[t]{2}{*}{ Sig. (p) } & \multirow[t]{2}{*}{ Odds ratio } & \multicolumn{2}{|c|}{ 95\% CI for odds ratio } \\
\hline & & & & & $\begin{array}{c}\text { Lower } \\
\text { limit }\end{array}$ & $\begin{array}{c}\text { Upper } \\
\text { limit }\end{array}$ \\
\hline \multicolumn{7}{|c|}{ Demographic characteristics } \\
\hline Age (in years) & -0.003 & 0.007 & 0.655 & 0.997 & 0.983 & 1.011 \\
\hline Males (ref: females) & 0.000 & 0.001 & 0.675 & 1.000 & 0.997 & 1.002 \\
\hline $\begin{array}{l}\text { Whites (ref: non- } \\
\text { whites) }\end{array}$ & -0.909 & 0.293 & 0.002 & 0.403 & 0.227 & 0.716 \\
\hline $\begin{array}{l}\text { Metro (ref: non- } \\
\text { metro) }\end{array}$ & 0.487 & 0.198 & 0.014 & 1.628 & 1.105 & 2.398 \\
\hline \multicolumn{7}{|c|}{ Prescribing physician type } \\
\hline $\begin{array}{l}\text { Psychiatric } \\
\text { prescriber }\end{array}$ & 0.000 & 0.000 & 0.034 & 1.000 & 0.999 & 1.000 \\
\hline \multicolumn{7}{|c|}{ Schizophrenia subtype (ref: only schizophrenia) } \\
\hline $\begin{array}{l}\text { Schizoaffective } \\
\text { disorder }\end{array}$ & 0.900 & 0.216 & $<.0001$ & 2.460 & 1.611 & 3.755 \\
\hline $\begin{array}{l}\text { Both schizoaffective } \\
\text { disorder and } \\
\text { schizophrenia }\end{array}$ & 0.772 & 0.212 & 0.000 & 2.163 & 1.427 & 3.280 \\
\hline \multicolumn{7}{|c|}{ Pre-index co-morbidities } \\
\hline $\begin{array}{l}\text { Alcohol and } \\
\text { substance abuse }\end{array}$ & -1.242 & 0.181 & $<.0001$ & 0.289 & 0.203 & 0.412 \\
\hline Bipolar disorder & -0.229 & 0.241 & 0.340 & 0.795 & 0.496 & 1.274 \\
\hline Major depression & -0.170 & 0.207 & 0.413 & 0.844 & 0.562 & 1.267 \\
\hline $\begin{array}{l}\text { Mild to Moderate } \\
\text { depression }\end{array}$ & -0.384 & 0.236 & 0.104 & 0.681 & 0.429 & 1.083 \\
\hline $\begin{array}{l}\text { Other mental } \\
\text { comorbidities }\end{array}$ & -0.395 & 0.183 & 0.031 & 0.674 & 0.470 & 0.965 \\
\hline diabetes & -0.504 & 0.266 & 0.058 & 0.604 & 0.359 & 1.017 \\
\hline
\end{tabular}


Table 27a: Logistic regression model for the impact of index antipsychotic on having a mental health-related ER episode among schizophrenia patients (contd.)

\begin{tabular}{|c|c|c|c|c|c|c|}
\hline & \multirow[t]{2}{*}{ Beta } & \multirow[t]{2}{*}{$S E$} & \multirow[t]{2}{*}{ Sig. $(p)$} & \multirow[t]{2}{*}{ Odds ratio } & \multicolumn{2}{|c|}{$95 \%$ CI for odds ratio } \\
\hline & & & & & $\begin{array}{c}\text { Lower } \\
\text { limit }\end{array}$ & $\begin{array}{l}\text { Upper } \\
\text { limit }\end{array}$ \\
\hline hyperlipedemia & 0.155 & 0.315 & 0.624 & 1.167 & 0.629 & 2.165 \\
\hline hypertension & -0.132 & 0.219 & 0.547 & 0.876 & 0.571 & 1.346 \\
\hline $\begin{array}{l}\text { Charlson } \\
\text { Comorbidity Index }\end{array}$ & -0.062 & 0.064 & 0.331 & 0.940 & 0.829 & 1.065 \\
\hline \multicolumn{7}{|c|}{ Pre-index period psychiatric medication use (days of supply) } \\
\hline Mood stabilizers & -0.001 & 0.001 & 0.053 & 0.999 & 0.998 & 1.000 \\
\hline Antipsychotics & 0.000 & 0.002 & 0.799 & 1.000 & 0.997 & 1.004 \\
\hline Antidepressants & 0.000 & 0.001 & 0.537 & 1.000 & 0.999 & 1.001 \\
\hline Benzodiazepines & -0.001 & 0.001 & 0.362 & 0.999 & 0.998 & 1.001 \\
\hline \multicolumn{7}{|c|}{ Pre-index healthcare utilization and cost } \\
\hline $\begin{array}{l}\text { Number of } \\
\text { psychotherapy visits }\end{array}$ & -0.015 & 0.016 & 0.367 & 0.985 & 0.955 & 1.017 \\
\hline $\begin{array}{l}\text { Number of } \\
\text { medication } \\
\text { management visits }\end{array}$ & 0.032 & 0.028 & 0.246 & 1.033 & 0.978 & 1.090 \\
\hline $\begin{array}{l}\text { Any pre-index } \\
\text { mental-health } \\
\text { related ER visits }\end{array}$ & -0.717 & 0.286 & 0.012 & 0.488 & 0.279 & 0.854 \\
\hline $\begin{array}{l}\text { Pre-index mental- } \\
\text { health related } \\
\text { physician visits }\end{array}$ & -0.020 & 0.054 & 0.710 & 0.980 & 0.882 & 1.089 \\
\hline $\begin{array}{l}\text { Any pre-index } \\
\text { mental-health } \\
\text { related }\end{array}$ & & & & & & \\
\hline hospitalizations & -0.308 & 0.171 & 0.072 & 0.735 & 0.525 & 1.028 \\
\hline \multicolumn{7}{|c|}{ Year of index prescription (ref: 1999) } \\
\hline - 2000 & 0.395 & 0.219 & 0.071 & 1.484 & 0.967 & 2.278 \\
\hline - 2001 & 0.349 & 0.254 & 0.169 & 1.418 & 0.862 & 2.331 \\
\hline
\end{tabular}


Table 27a: Logistic regression model for the impact of index antipsychotic on having a mental health-related ER episode among schizophrenia patients (contd.)

\begin{tabular}{lcccccc}
\hline & Beta & SE & Sig. (p) & Odds ratio & \multicolumn{2}{c}{ 95\% CI for odds ratio } \\
\cline { 5 - 6 } & & & & & $\begin{array}{c}\text { Lower } \\
\text { limit }\end{array}$ & $\begin{array}{c}\text { Upper } \\
\text { limit }\end{array}$ \\
\hline & & & & & & \\
Index prescription (ref: quetiapine) & & & & & \\
Risperidone & 0.211 & 0.271 & 0.437 & 1.234 & 0.726 & 2.100 \\
Olanzapine & 0.118 & 0.240 & 0.623 & 1.125 & 0.703 & 1.800 \\
Typicals & -0.100 & 0.258 & 0.698 & 0.905 & 0.546 & 1.499 \\
\hline
\end{tabular}

\section{Model fit statistics:}

-2 Log Likehood $=1057.6 \quad$, chi-square $=178.25 \quad, p<0.0001$ 
TWO-PART MODEL-PART 2

Table 27b: Ordinary Least Squares (OLS) regression model for the impact of index antipsychotic on mental healthcare-related ER cost among schizophrenia patients

\begin{tabular}{|c|c|c|c|c|}
\hline & Beta & S.E & t-statistic & $\begin{array}{c}\text { Significance } \\
\text { (p) }\end{array}$ \\
\hline \multicolumn{5}{|l|}{ Demographic characteristics } \\
\hline Age (in years) & -0.006 & 0.007 & -0.750 & 0.456 \\
\hline Males (ref: females) & 0.000 & 0.001 & -0.200 & 0.844 \\
\hline Whites (ref: non-whites) & -0.076 & 0.306 & -0.250 & 0.803 \\
\hline Metro (ref: non-metro) & 0.158 & 0.188 & 0.840 & 0.402 \\
\hline \multicolumn{5}{|l|}{ Prescribing physician type } \\
\hline Psychiatric prescriber & 0.000 & 0.000 & -0.520 & 0.606 \\
\hline \multicolumn{5}{|c|}{ Schizophrenia subtype (ref: only schizophrenia) } \\
\hline Schizoaffective disorder & -0.063 & 0.205 & -0.310 & 0.759 \\
\hline $\begin{array}{l}\text { Both schizoaffective disorder } \\
\text { and schizophrenia }\end{array}$ & -0.256 & 0.199 & -1.290 & 0.199 \\
\hline \multicolumn{5}{|l|}{ Pre-index co-morbidities } \\
\hline Alcohol and substance abuse & 0.281 & 0.157 & 1.790 & 0.074 \\
\hline Bipolar disorder & 0.096 & 0.207 & 0.460 & 0.645 \\
\hline Major depression & 0.368 & 0.191 & 1.930 & 0.055 \\
\hline Mild to Moderate depression & 0.244 & 0.206 & 1.190 & 0.236 \\
\hline Other mental comorbidities & 0.035 & 0.172 & 0.200 & 0.840 \\
\hline Diabetes & 0.435 & 0.245 & 1.780 & 0.077 \\
\hline Hyperlipedemia & -0.177 & 0.302 & -0.590 & 0.558 \\
\hline Hypertension & 0.106 & 0.205 & 0.520 & 0.606 \\
\hline Charlson Comorbidity Index & 0.155 & 0.074 & 2.100 & 0.037 \\
\hline \multicolumn{5}{|c|}{ Pre-index period psychiatric medication use (days of supply) } \\
\hline Mood stabilizers & 0.000 & 0.001 & 0.380 & 0.706 \\
\hline Antipsychotics & -0.003 & 0.002 & -1.450 & 0.147 \\
\hline Antidepressants & 0.000 & 0.001 & 0.850 & 0.395 \\
\hline Benzodiazepines & -0.001 & 0.001 & -1.800 & 0.073 \\
\hline $\begin{array}{l}\text { Number of psychotherapy } \\
\text { visits }\end{array}$ & -0.031 & 0.012 & -2.730 & 0.007 \\
\hline
\end{tabular}


Table 27b: Ordinary Least Squares (OLS) regression model for the impact of index antipsychotic on mental healthcare-related ER cost among schizophrenia patients (contd.)

\begin{tabular}{|c|c|c|}
\hline Beta & S.E & t-statistic \\
\hline
\end{tabular}

\section{Pre-index healthcare utilization and cost}

Number of medication

management visits

$\begin{array}{llll}-0.010 & 0.028 & -0.350 & 0.723\end{array}$

Number of pre-index mental-

health related ER visits

$-0.030$

0.109

$-0.280$

0.782

Pre-index mental-health

related physician visits

$\begin{array}{llll}-0.019 & 0.044 & -0.440 & 0.663\end{array}$

Number of pre-index mental-

health related hospitalizations

0.150

0.250

0.460

0.651

Pre-index mental health-

related cost

0.006

0.012

0.460

0.644

Year of index prescription (ref: 1999)

- 2000

0.234

0.202

1.160

0.248

- 2001

0.071

0.233

0.310

0.760

Index prescription (ref: quetiapine)

$\begin{array}{lllll}\text { Risperidone } & -0.535 & 0.259 & -2.060 & 0.060 \\ \text { Olanzapine } & -0.390 & 0.227 & -1.720 & 0.086 \\ \text { Typicals } & -0.375 & 0.239 & -1.570 & 0.118\end{array}$

Model fit statistics:

Adjusted R-square $=7.26 \%, F=1.8, \mathrm{p}<0.0001$ 
Table 28: Ordinary Least Squares (OLS) regression model for the impact of index antipsychotic on mental healthcare-related pharmacy cost among schizophrenia patients

Beta S.E t-statistic Significance

(p)

Demographic characteristics

Age (in years)

Males (ref: females)

Whites (ref: non-whites)

Metro (ref: non-metro)

\subsection{1}

0.000

$-0.007$

$-0.042$

Prescribing physician type

Psychiatric prescriber

0.000

0.000

5.26

$<.0001$

Schizophrenia subtype (ref: only schizophrenia)

Schizoaffective disorder

$$
0.006
$$

0.093

0.07

0.946

Both schizoaffective disorder

and schizophrenia

$-0.222$

0.097

$-2.29$

0.022

\section{Pre-index co-morbidities}

\begin{tabular}{lcccc} 
Alcohol and substance abuse & -0.139 & 0.086 & -1.61 & 0.107 \\
Bipolar disorder & 0.005 & 0.113 & 0.04 & 0.966 \\
Major depression & 0.056 & 0.095 & 0.58 & 0.560 \\
Mild to Moderate depression & 0.010 & 0.112 & 0.09 & 0.927 \\
Other mental comorbidities & 0.027 & 0.083 & 0.33 & 0.742 \\
Diabetes & 0.098 & 0.127 & 0.77 & 0.444 \\
Hyperlipedemia & 0.010 & 0.141 & 0.07 & 0.945 \\
Hypertension & -0.040 & 0.102 & -0.4 & 0.692 \\
Charlson Comorbidity Index & -0.066 & 0.030 & -2.19 & 0.029 \\
Pre-index period psychiatric medication use (days of supply) & \\
Mood stabilizers & 0.091 & 0.000 & 5.45 & $<.0001$ \\
Antipsychotics & 0.001 & 0.001 & 1.36 & 0.174 \\
Antidepressants & 0.072 & 0.000 & 5.14 & $<.0001$ \\
Benzodiazepines & 0.034 & 0.000 & 2.82 & 0.005 \\
\hline
\end{tabular}


Table 28: Ordinary Least Squares (OLS) regression model for the impact of index antipsychotic on mental healthcare-related pharmacy cost among schizophrenia patients

\begin{tabular}{|c|c|c|}
\hline Beta & S.E & t-statistic \\
\hline
\end{tabular}

\section{Pre-index healthcare utilization and cost}

Number of psychotherapy

visits

$0.001 \quad 0.008 \quad 0.13$

0.894

Number of medication

management visits

$\begin{array}{llll}-0.010 & 0.012 & -0.88 & 0.380\end{array}$

Number of pre-index mental-

health related ER visits

$\begin{array}{llll}-0.038 & 0.073 & -0.53 & 0.599\end{array}$

Pre-index mental-health

related physician visits

$\begin{array}{llll}-0.00073 & 0.025 & -0.03 & 0.976\end{array}$

Number of pre-index mental-

health related hospitalizations

$\begin{array}{llll}0.043 & 0.036 & 1.18 & 0.237\end{array}$

Pre-index psychiatric drug cost

0.003

0.005

0.54

0.586

Year of index prescription (ref: 1999)

- 2000

$-0.043$

0.095

$-0.45$

0.654

- 2001

0.022

0.110

0.2

0.845

Index prescription (ref: quetiapine)
Risperidone
0.032
0.121
0.26
0.794
Olanzapine
0.246
0.108
2.28
0.023
Typicals
$-0.775$
0.116
$-6.65$
$<.0001$

\section{Model fit statistics:}

Adjusted R-square $=27.78 \% \quad, F=12.00, p<0.0001$ 
Table 29: Ordinary Least Squares (OLS) regression model for the impact of index antipsychotic on mental healthcare-related outpatient cost among schizophrenia patients

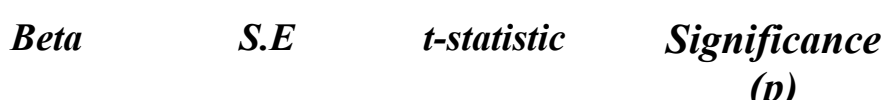

Demographic characteristics

Age (in years)

Males (ref: females)

Whites (ref: non-whites)

Metro (ref: non-metro)

\subsection{9}

0.004

0.203

$-0.637$

Prescribing physician type

Psychiatric prescriber

0.001

0.000

2.76

0.006

Schizophrenia subtype (ref: only schizophrenia)

Schizoaffective disorder

Both schizoaffective disorder

and schizophrenia

Pre-index co-morbidities

\begin{tabular}{lcccc} 
Alcohol and substance abuse & 0.991 & 0.366 & 2.71 & 0.007 \\
Bipolar disorder & 0.626 & 0.476 & 1.31 & 0.189 \\
Major depression & -0.083 & 0.400 & -0.21 & 0.836 \\
Mild to Moderate depression & 0.626 & 0.473 & 1.32 & 0.186 \\
Other mental comorbidities & -1.166 & 0.351 & -3.32 & 0.001 \\
diabetes & 0.182 & 0.536 & 0.34 & 0.734 \\
hyperlipedemia & -0.277 & 0.593 & -0.47 & 0.641 \\
hypertension & -0.210 & 0.428 & -0.49 & 0.624 \\
Charlson Comorbidity Index & -0.193 & 0.127 & -1.52 & 0.129 \\
Pre-index period psychiatric medication use (days of supply) & \\
Mood stabilizers & -0.001 & 0.001 & -0.85 & 0.396 \\
Antipsychotics & -0.002 & 0.004 & -0.64 & 0.520 \\
Antidepressants & 0.000 & 0.001 & -0.24 & 0.811 \\
Benzodiazepines & -1.318 & 0.490 & 2.69 & 0.007 \\
\hline
\end{tabular}


Table 29: Ordinary Least Squares (OLS) regression model for the impact of index antipsychotic on mental healthcare-related outpatient cost among schizophrenia patients (contd.)

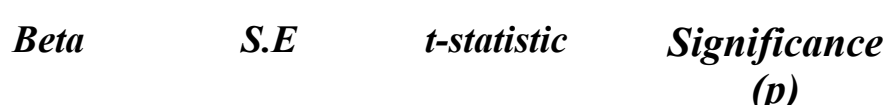

\section{Pre-index healthcare utilization and cost}

Number of psychotherapy

visits

$$
0.000
$$

0.033

0.01

0.990

Number of medication

management visits

0.082

0.051

1.6

0.110

Number of pre-index mental-

health related ER visits

$-0.207$

0.306

$-0.68$

0.499

Pre-index mental-health

related physician visits

$-0.064$

0.106

$-0.6$

0.547

Number of pre-index mental-

health related hospitalizations

0.320

0.153

2.09

0.037

Pre-index outpatient drug cost

0.365

0.025

14.51

$<.0001$

Year of index prescription (ref: 1999)

- 2000

$-0.047$

0.401

$-0.12$

0.907

- 2001

$-0.365$

0.465

$-0.79$

0.432

Index prescription (ref: quetiapine)

$\begin{array}{lcccc}\text { Risperidone } & -0.540 & 0.508 & -1.06 & 0.288 \\ \text { Olanzapine } & -0.412 & 0.455 & -0.91 & 0.365 \\ \text { Typicals } & 0.03 & 0.001 & 2.3 & 0.022\end{array}$

\section{Model fit statistics:}

Adjusted R-square $=35.91 \%, F=19.03, p<0.0001$ 
TWO-PART MODEL-PART 1

Table 30a: Logistic regression model for the impact of index antipsychotic on having a mental health-related hospitalization episode

\begin{tabular}{|c|c|c|c|c|c|c|}
\hline & \multirow[t]{2}{*}{ Beta } & \multirow[t]{2}{*}{$S E$} & \multirow[t]{2}{*}{ Sig. (p) } & \multirow[t]{2}{*}{ Odds ratio } & \multicolumn{2}{|c|}{ 95\% CI for odds ratio } \\
\hline & & & & & Lower & Upper \\
\hline \multicolumn{7}{|c|}{ Demographic characteristics } \\
\hline Age (in years) & -0.012 & 0.008 & 0.121 & 0.988 & 0.972 & 1.003 \\
\hline $\begin{array}{l}18 \text { years or above } \\
\text { (ref: less than } 18 \\
\text { years) }\end{array}$ & -0.277 & 0.241 & 0.250 & 0.758 & 0.473 & 1.215 \\
\hline Males (ref: females) & -0.005 & 0.008 & 0.533 & 0.995 & 0.979 & 1.011 \\
\hline $\begin{array}{l}\text { Whites (ref: non- } \\
\text { whites) }\end{array}$ & -0.763 & 0.350 & 0.029 & 0.466 & 0.235 & 0.927 \\
\hline $\begin{array}{l}\text { Metro (ref: non- } \\
\text { metro) }\end{array}$ & 0.421 & 0.230 & 0.068 & 1.523 & 0.970 & 2.391 \\
\hline \multicolumn{7}{|c|}{ Prescribing physician type } \\
\hline $\begin{array}{l}\text { Psychiatric } \\
\text { prescriber }\end{array}$ & 0.000 & 0.000 & 0.506 & 1.000 & 0.999 & 1.000 \\
\hline \multicolumn{7}{|c|}{ Bipolar disorder subtype (ref: Bipolar disorder I) } \\
\hline Bipolar disorder II & 0.448 & 0.241 & 0.064 & 1.565 & 0.975 & 2.511 \\
\hline Mixed & 0.835 & 0.243 & 0.001 & 2.305 & 1.432 & 3.712 \\
\hline \multicolumn{7}{|c|}{ Pre-index co-morbidities } \\
\hline $\begin{array}{l}\text { Alcohol and } \\
\text { substance abuse }\end{array}$ & -0.889 & 0.206 & $<.0001$ & 0.411 & 0.275 & 0.615 \\
\hline Schizophrenia & -0.228 & 0.275 & 0.407 & 0.796 & 0.464 & 1.364 \\
\hline Major depression & -0.153 & 0.232 & 0.509 & 0.858 & 0.545 & 1.351 \\
\hline $\begin{array}{l}\text { Mild to Moderate } \\
\text { depression }\end{array}$ & -0.069 & 0.268 & 0.797 & 0.933 & 0.552 & 1.578 \\
\hline $\begin{array}{l}\text { Other mental } \\
\text { comorbidities }\end{array}$ & -0.192 & 0.207 & 0.354 & 0.825 & 0.550 & 1.238 \\
\hline Diabetes & -0.363 & 0.297 & 0.221 & 0.696 & 0.389 & 1.244 \\
\hline Hyperlipedemia & 0.228 & 0.359 & 0.525 & 1.256 & 0.622 & 2.536 \\
\hline Hypertension & 0.219 & 0.246 & 0.375 & 1.245 & 0.768 & 2.017 \\
\hline
\end{tabular}


Table 30a: Logistic regression model for the impact of index antipsychotic on having a mental health-related hospitalization episode (contd.)

\begin{tabular}{|c|c|c|c|c|c|c|}
\hline & \multirow[t]{2}{*}{ Beta } & \multirow[t]{2}{*}{$S E$} & \multirow[t]{2}{*}{ Sig. (p) } & \multirow[t]{2}{*}{ Odds ratio } & \multicolumn{2}{|c|}{ 95\% CI for odds ratio } \\
\hline & & & & & Lower & Upper \\
\hline Charlson & & & 0.004 & & & \\
\hline Comorbidity Index & -0.189 & 0.066 & & 0.828 & 0.727 & 0.942 \\
\hline \multicolumn{7}{|c|}{ Pre-index period psychiatric medication use (days of supply) } \\
\hline Mood stabilizers & 0.000 & 0.001 & 0.731 & 1.000 & 0.998 & 1.001 \\
\hline Antipsychotics & -0.002 & 0.002 & 0.302 & 0.998 & 0.994 & 1.002 \\
\hline Antidepressants & -0.001 & 0.001 & 0.083 & 0.999 & 0.998 & 1.000 \\
\hline Benzodiazepines & -0.001 & 0.001 & 0.459 & 0.999 & 0.998 & 1.001 \\
\hline \multicolumn{7}{|c|}{ Pre-index healthcare utilization and cost } \\
\hline $\begin{array}{l}\text { Number of } \\
\text { psychotherapy visits }\end{array}$ & -0.001 & 0.024 & 0.977 & 0.999 & 0.953 & 1.048 \\
\hline $\begin{array}{l}\text { Number of } \\
\text { medication } \\
\text { management visits }\end{array}$ & 0.100 & 0.036 & 0.006 & 1.105 & 1.029 & 1.186 \\
\hline $\begin{array}{l}\text { Any pre-index } \\
\text { mental-health } \\
\text { related ER visits }\end{array}$ & -0.190 & 0.321 & 0.553 & 0.827 & 0.441 & 1.550 \\
\hline $\begin{array}{l}\text { Pre-index mental- } \\
\text { health related } \\
\text { physician visits }\end{array}$ & 0.028 & 0.063 & 0.653 & 1.029 & 0.910 & 1.163 \\
\hline $\begin{array}{l}\text { Any pre-index } \\
\text { mental-health } \\
\text { related } \\
\text { hospitalizations }\end{array}$ & -0.749 & 0.190 & $<.0001$ & 0.473 & 0.326 & 0.686 \\
\hline \multicolumn{7}{|c|}{ Year of index prescription (ref: 1999) } \\
\hline - 2000 & -0.198 & 0.233 & 0.396 & 0.821 & 0.520 & 1.296 \\
\hline - 2001 & 0.233 & 0.287 & 0.417 & 1.263 & 0.719 & 2.216 \\
\hline \multicolumn{7}{|c|}{ Index prescription (ref: quetiapine) } \\
\hline Risperidone & 0.268 & 0.316 & 0.397 & 1.307 & 0.704 & 2.426 \\
\hline Olanzapine & 0.082 & 0.286 & 0.774 & 1.086 & 0.620 & 1.903 \\
\hline Typicals & 0.078 & 0.315 & 0.806 & 1.081 & 0.583 & 2.003 \\
\hline
\end{tabular}

Model fit statistics:

$\overline{\mathbf{- 2} \log \text { Likehood }}=\quad 845.24$, chi-square $=136.88, \mathrm{p}=0.00$ 
TWO-PART MODEL-PART 2

Table 30b: Ordinary Least Squares (OLS) regression model for the impact of index antipsychotic on mental healthcare-related hospitalization cost among bipolar disorder patients

$\begin{array}{ccc}\text { Beta S.E t-statistic } & \begin{array}{l}\text { Significance } \\ (p)\end{array}\end{array}$

Demographic characteristics

Age (in years)

0.005

0.005

0.930

0.354

18 years or above (ref: less

than 18 years)

0.100

0.163

0.610

0.541

Males (ref: females)

$-0.030$

0.119

$-0.250$

0.801

Whites (ref: non-whites)

0.484

0.245

1.970

0.050

Metro (ref: non-metro)

0.082

0.158

0.520

0.606

Prescribing physician type

Psychiatric prescriber

$0.000 \quad 0.000$

$-0.730$

0.465

Bipolar disorder subtype (ref: Bipolar disorder I)

$\begin{array}{lllll}\text { Bipolar disorder II } & -0.056 & 0.151 & -0.370 & 0.711 \\ \text { Mixed } & -0.181 & 0.206 & -0.880 & 0.382 \\ \text { Pre-index co-morbidities } & & & & \\ \text { Alcohol and substance abuse } & 0.318 & 0.123 & 2.590 & 0.010 \\ \text { Bipolar disorder } & 0.031 & 0.166 & 0.180 & 0.854 \\ \text { Major depression } & 0.171 & 0.143 & 1.200 & 0.232 \\ \text { Mild to Moderate depression } & 0.039 & 0.160 & 0.250 & 0.806 \\ \text { Other mental comorbidities } & 0.125 & 0.130 & 0.970 & 0.335 \\ \text { diabetes } & -0.043 & 0.170 & -0.250 & 0.800 \\ \text { hyperlipedemia } & -0.190 & 0.219 & -0.870 & 0.387 \\ \text { hypertension } & 0.179 & 0.152 & 1.180 & 0.241 \\ \text { Charlson Comorbidity Index } & 0.044 & 0.042 & 1.050 & 0.295\end{array}$

Pre-index period psychiatric medication use (days of supply)

$\begin{array}{lllll}\text { Mood stabilizers } & -0.021 & 0.000 & -1.670 & 0.096 \\ \text { Antipsychotics } & 0.000 & 0.001 & 0.380 & 0.702 \\ \text { Antidepressants } & 0.000 & 0.000 & -0.320 & 0.750 \\ \text { Benzodiazepines } & 0.000 & 0.000 & -0.630 & 0.528\end{array}$


Table 30b: Ordinary Least Squares (OLS) regression model for the impact of index antipsychotic on mental healthcare-related hospitalization cost among bipolar disorder patients

$$
\begin{array}{llll}
\text { Beta S.E } & \text { t-statistic } & \begin{array}{l}
\text { Significance } \\
(p)
\end{array}
\end{array}
$$

\begin{tabular}{|c|c|c|c|c|}
\hline \multicolumn{5}{|c|}{ Pre-index healthcare utilization and cost } \\
\hline $\begin{array}{l}\text { Number of psychotherapy } \\
\text { visits }\end{array}$ & 0.013 & 0.014 & 0.920 & 0.357 \\
\hline $\begin{array}{l}\text { Number of medication } \\
\text { management visits }\end{array}$ & -0.020 & 0.026 & -0.770 & 0.445 \\
\hline $\begin{array}{l}\text { Number of pre-index mental- } \\
\text { health related ER visits }\end{array}$ & -0.008 & 0.078 & -0.100 & 0.919 \\
\hline $\begin{array}{l}\text { Pre-index mental-health } \\
\text { related physician visits }\end{array}$ & -0.048 & 0.037 & -1.300 & 0.195 \\
\hline $\begin{array}{l}\text { Number of pre-index mental- } \\
\text { health related hospitalizations }\end{array}$ & 0.094 & 0.206 & 0.460 & 0.647 \\
\hline $\begin{array}{l}\text { Pre-index mental health- } \\
\text { related cost }\end{array}$ & 0.020 & 0.007 & 2.960 & 0.004 \\
\hline \multicolumn{5}{|c|}{ Year of index prescription (ref: 1999) } \\
\hline - 2000 & 0.300 & 0.143 & 2.110 & 0.036 \\
\hline - 2001 & -0.186 & 0.184 & -1.010 & 0.314 \\
\hline \multicolumn{5}{|c|}{ Index prescription (ref: quetiapine) } \\
\hline Risperidone & -0.201 & 0.200 & -1.010 & 0.315 \\
\hline Olanzapine & -0.414 & 0.184 & -2.250 & 0.225 \\
\hline Typicals & -0.343 & 0.201 & -1.710 & 0.089 \\
\hline
\end{tabular}

\section{Model fit statistics:}

Adjusted R-square $=12.26 \%, \mathbf{F}=2.01, \mathbf{p}=0.000$ 
TWO-PART MODEL-PART 1

Table 31a: Logistic regression model for the impact of index antipsychotic on having a mental health-related ER episode among bipolar disorder patients

\begin{tabular}{|c|c|c|c|c|c|c|}
\hline & \multirow[t]{2}{*}{ Beta } & \multirow[t]{2}{*}{$S E$} & \multirow[t]{2}{*}{ Sig. (p) } & \multirow[t]{2}{*}{ Odds ratio } & \multicolumn{2}{|c|}{ 95\% CI for odds ratio } \\
\hline & & & & & $\begin{array}{l}\text { Lower } \\
\text { Limit }\end{array}$ & $\begin{array}{l}\text { Upper } \\
\text { Limit }\end{array}$ \\
\hline \multicolumn{7}{|c|}{ Demographic characteristics } \\
\hline Age (in years) & -0.003 & 0.008 & 0.709 & 0.997 & 0.982 & 1.013 \\
\hline $\begin{array}{l}18 \text { years or above } \\
\text { (ref: less than } 18 \\
\text { years) }\end{array}$ & -0.526 & 0.242 & 0.030 & 0.591 & 0.368 & 0.950 \\
\hline Males (ref: females) & 0.000 & 0.001 & 0.919 & 1.000 & 0.997 & 1.002 \\
\hline $\begin{array}{l}\text { Whites (ref: non- } \\
\text { whites) }\end{array}$ & -0.866 & 0.342 & 0.011 & 0.421 & 0.215 & 0.823 \\
\hline $\begin{array}{l}\text { Metro (ref: non- } \\
\text { metro) }\end{array}$ & 0.487 & 0.230 & 0.035 & 1.627 & 1.036 & 2.554 \\
\hline \multicolumn{7}{|c|}{ Prescribing physician type } \\
\hline $\begin{array}{l}\text { Psychiatric } \\
\text { prescriber }\end{array}$ & 0.000 & 0.000 & 0.164 & 1.000 & 0.999 & 1.000 \\
\hline \multicolumn{7}{|c|}{ Bipolar disorder subtype (ref: bipolar disorder I) } \\
\hline Bipolar disorder I & 0.827 & 0.247 & 0.001 & 2.287 & 1.408 & 3.713 \\
\hline Mixed & 0.846 & 0.237 & 0.000 & 2.330 & 1.465 & 3.706 \\
\hline \multicolumn{7}{|c|}{ Pre-index co-morbidities } \\
\hline $\begin{array}{l}\text { Alcohol and } \\
\text { substance abuse }\end{array}$ & -1.093 & 0.204 & $<.0001$ & 0.335 & 0.225 & 0.500 \\
\hline Schizophrenia & -0.332 & 0.266 & 0.211 & 0.717 & 0.426 & 1.207 \\
\hline Major depression & -0.181 & 0.228 & 0.429 & 0.835 & 0.534 & 1.306 \\
\hline $\begin{array}{l}\text { Mild to Moderate } \\
\text { depression }\end{array}$ & -0.217 & 0.262 & 0.408 & 0.805 & 0.481 & 1.346 \\
\hline $\begin{array}{l}\text { Other mental } \\
\text { comorbidities }\end{array}$ & -0.420 & 0.203 & 0.038 & 0.657 & 0.442 & 0.977 \\
\hline diabetes & -0.513 & 0.293 & 0.080 & 0.598 & 0.337 & 1.063 \\
\hline hyperlipedemia & 0.100 & 0.345 & 0.771 & 1.106 & 0.562 & 2.175 \\
\hline hypertension & -0.144 & 0.237 & 0.542 & 0.866 & 0.544 & 1.376 \\
\hline
\end{tabular}


Table 31a: Logistic regression model for the impact of index antipsychotic on having a mental health-related ER episode among bipolar disorder patients (contd.)

\begin{tabular}{|c|c|c|c|c|c|c|}
\hline & \multirow[t]{3}{*}{ Beta } & \multirow[t]{3}{*}{$S E$} & \multirow[t]{3}{*}{ Sig. $(p)$} & \multirow[t]{3}{*}{ Odds ratio } & \multicolumn{2}{|c|}{ 95\% CI for odds ratio } \\
\hline & & & & & Lower & Upper \\
\hline & & & & & Limit & Limit \\
\hline Charlson & & & & & 0.836 & \\
\hline Comorbidity Index & -0.047 & 0.067 & 0.481 & 0.954 & & 1.088 \\
\hline \multicolumn{7}{|c|}{ Pre-index period psychiatric medication use (days of supply) } \\
\hline Mood stabilizers & -0.001 & 0.001 & 0.130 & 0.999 & 0.998 & 1.000 \\
\hline Antipsychotics & 0.002 & 0.002 & 0.502 & 1.002 & 0.997 & 1.006 \\
\hline Antidepressants & 0.000 & 0.001 & 0.377 & 1.000 & 0.998 & 1.001 \\
\hline Benzodiazepines & -0.001 & 0.001 & 0.353 & 0.999 & 0.998 & 1.001 \\
\hline \multicolumn{7}{|c|}{ Pre-index healthcare utilization and cost } \\
\hline $\begin{array}{l}\text { Number of } \\
\text { psychotherapy visits }\end{array}$ & -0.010 & 0.021 & 0.644 & 0.990 & 0.950 & 1.032 \\
\hline $\begin{array}{l}\text { Number of } \\
\text { medication }\end{array}$ & & & & & & \\
\hline management visits & 0.033 & 0.032 & 0.300 & 1.034 & 0.971 & 1.100 \\
\hline $\begin{array}{l}\text { Any pre-index } \\
\text { mental-health }\end{array}$ & & & & & & \\
\hline related ER visits & -0.758 & 0.314 & 0.016 & 0.469 & 0.253 & 0.868 \\
\hline $\begin{array}{l}\text { Pre-index mental- } \\
\text { health related } \\
\text { physician visits }\end{array}$ & -0.057 & 0.060 & 0.346 & 0.945 & 0.840 & 1.063 \\
\hline $\begin{array}{l}\text { Any pre-index } \\
\text { mental-health } \\
\text { related }\end{array}$ & & & & & & \\
\hline hospitalizations & -0.403 & 0.190 & 0.034 & 0.668 & 0.460 & 0.971 \\
\hline \multicolumn{7}{|c|}{ Year of index prescription (ref: 1999) } \\
\hline - 2000 & 0.214 & 0.234 & 0.360 & 1.239 & 0.783 & 1.959 \\
\hline - 2001 & 0.297 & 0.277 & 0.284 & 1.346 & 0.782 & 2.317 \\
\hline \multicolumn{7}{|c|}{ Index prescription (ref: quetiapine) } \\
\hline Risperidone & 0.161 & 0.308 & 0.601 & 1.175 & 0.642 & 2.150 \\
\hline Olanzapine & 0.044 & 0.281 & 0.876 & 1.045 & 0.602 & 1.813 \\
\hline Typicals & -0.215 & 0.310 & 0.489 & 0.807 & 0.439 & 1.482 \\
\hline
\end{tabular}


Model fit statistics:

-2 Log Likehood $=866.29 \quad$, chi-square $=142.48 \quad, p<0.0001$ 
TWO-PART MODEL-PART 2

Table 31b: Ordinary Least Squares (OLS) regression model for the impact of index antipsychotic on mental healthcare-related ER cost among bipolar disorder patients

\begin{tabular}{|c|c|c|c|c|}
\hline & Beta & S.E & t-statistic & $\begin{array}{c}\text { Significance } \\
(p)\end{array}$ \\
\hline \multicolumn{5}{|l|}{ Demographic characteristics } \\
\hline Age (in years) & 0.001 & 0.008 & 0.180 & 0.858 \\
\hline $\begin{array}{l}18 \text { years or above (ref: less } \\
\text { than } 18 \text { years) }\end{array}$ & 0.197 & 0.243 & 0.810 & 0.419 \\
\hline Males (ref: females) & 0.000 & 0.001 & 0.170 & 0.863 \\
\hline Whites (ref: non-whites) & -0.129 & 0.346 & -0.370 & 0.709 \\
\hline Metro (ref: non-metro) & -0.038 & 0.222 & -0.170 & 0.864 \\
\hline \multicolumn{5}{|l|}{ Prescribing physician type } \\
\hline Psychiatric prescriber & 0.000 & 0.000 & -0.730 & 0.465 \\
\hline \multicolumn{5}{|c|}{ Bipolar disorder subtype (ref: Bipolar disorder I) } \\
\hline Bipolar disorder I & -0.056 & 0.231 & -0.240 & 0.807 \\
\hline Mixed & -0.488 & 0.216 & -2.260 & 0.025 \\
\hline \multicolumn{5}{|l|}{ Pre-index co-morbidities } \\
\hline Alcohol and substance abuse & 0.382 & 0.175 & 2.180 & 0.030 \\
\hline Schizophrenia & 0.115 & 0.232 & 0.500 & 0.619 \\
\hline Major depression & 0.402 & 0.206 & 1.950 & 0.052 \\
\hline Mild to Moderate depression & 0.307 & 0.231 & 1.330 & 0.186 \\
\hline Other mental comorbidities & -0.118 & 0.193 & -0.610 & 0.539 \\
\hline diabetes & 0.466 & 0.258 & 1.810 & 0.072 \\
\hline hyperlipedemia & -0.370 & 0.320 & -1.160 & 0.249 \\
\hline hypertension & 0.041 & 0.217 & 0.190 & 0.849 \\
\hline Charlson Comorbidity Index & 0.158 & 0.080 & 1.970 & 0.050 \\
\hline \multicolumn{5}{|c|}{ Pre-index period psychiatric medication use (days of supply) } \\
\hline Mood stabilizers & 0.051 & 0.001 & 1.170 & 0.244 \\
\hline Antipsychotics & -0.004 & 0.002 & -1.560 & 0.121 \\
\hline Antidepressants & 0.004 & 0.001 & 0.340 & 0.732 \\
\hline Benzodiazepines & -0.045 & 0.001 & -1.550 & 0.123 \\
\hline
\end{tabular}


Table 31b: Ordinary Least Squares (OLS) regression model for the impact of index antipsychotic on mental healthcare-related ER cost among bipolar disorder patients (contd.)

\begin{tabular}{|c|c|c|c|}
\hline Beta & S.E & t-statistic & $\begin{array}{c}\text { Significance } \\
\text { (p) }\end{array}$ \\
\hline
\end{tabular}

\section{Pre-index healthcare utilization and cost}

Number of psychotherapy

visits

$$
-0.029
$$

0.016

$-1.850$

0.065

Number of medication

management visits

$$
-0.007
$$

0.032

$-0.210$

0.831

Number of pre-index mental-

health related ER visits

$-0.040$

0.114

$-0.350$

0.726

Pre-index mental-health

related physician visits

$-0.021$

0.049

$-0.440$

0.663

Number of pre-index mental-

health related hospitalizations

0.028

0.063

0.650

0.910

Pre-index mental health-

related cost

0.000

0.013

0.030

0.978

Year of index prescription (ref: 1999)
- 2000
0.229
0.211
1.080
0.280
- 2001
0.005
0.257
0.020
0.984

Index prescription (ref: quetiapine)

Risperidone

Olanzapine

Typicals
$-0.681$

$-0.494$

$-0.436$

0.293

$-2.320$

0.071

0.264

$-1.870$

0.063

0.287

$-1.520$

0.130

\section{Model fit statistics:}

Adjusted R-square $=19.95 \%, F=2.47, p<0.000$ 
Table 32: Ordinary Least Squares (OLS) regression model for the impact of index antipsychotic on mental healthcare-related pharmacy cost among bipolar disorder patients

Beta $\quad$ S.E $\quad$-statistic Significance (p)

Demographic characteristics

\begin{tabular}{|c|c|c|c|c|}
\hline Age (in years) & 0.001 & 0.003 & 0.37 & 0.714 \\
\hline $\begin{array}{l}18 \text { years or above (ref: les } \\
\text { years) }\end{array}$ & 0.034 & 0.101 & 0.34 & 0.735 \\
\hline Males (ref: females) & 0.000 & 0.001 & 0.66 & 0.507 \\
\hline Whites (ref: non-whites) & 0.026 & 0.134 & 0.19 & 0.848 \\
\hline Metro (ref: non-metro) & -0.005 & 0.102 & -0.05 & 0.961 \\
\hline \multicolumn{5}{|l|}{ Prescribing physician type } \\
\hline Psychiatric prescriber & 0.000 & 0.000 & 466 & 000 \\
\hline
\end{tabular}

Bipolar disorder subtype (ref: bipolar disorder I)

Bipolar disorder I

$\begin{array}{llll}0.039 & 0.108 & 0.36 & 0.717 \\ -0.249 & 0.107 & -2.32 & 0.021\end{array}$

Mixed

\section{Pre-index co-morbidities}

Alcohol and substance abuse

Schizophrenia

$\begin{array}{cccc}-0.198 & 0.099 & -2 & 0.045 \\ -0.069 & 0.127 & -0.54 & 0.590 \\ 0.079 & 0.106 & 0.74 & 0.458\end{array}$

Major depression

0.079

0.106

0.74

0.458

Mild to Moderate depression

$-0.022$

0.125

$-0.17$

0.863

Other mental comorbidities

0.084

0.092

0.92

0.360

Diabetes

0.124

0.142

0.88

0.381

Hyperlipedemia

0.025

0.158

0.16

0.876

Hypertension

$-0.092$

0.112

$-0.82$

0.411

Charlson Comorbidity Index

$-0.044$

0.032

$-1.4$

0.161

Pre-index period psychiatric medication use (days of supply)

$\begin{array}{lcccc}\text { Mood stabilizers } & 0.041 & 0.000 & 4.73 & <.0001 \\ \text { Antipsychotics } & 0.001 & 0.001 & 0.61 & 0.539 \\ \text { Antidepressants } & 0.045 & 0.000 & 5.3 & <.0001 \\ \text { Benzodiazepines } & 0.032 & 0.000 & 2.34 & 0.020\end{array}$


Table 32: Ordinary Least Squares (OLS) regression model for the impact of index antipsychotic on mental healthcare-related pharmacy cost among bipolar disorder patients (contd.)

Beta S.E t-statistic Significance (p)

Pre-index healthcare utilization and cost

Number of psychotherapy visits

$\begin{array}{llll}0.016 & 0.010 & 1.61 & 0.108\end{array}$

Number of medication management visits

$\begin{array}{llll}-0.026 & 0.013 & -1.93 & 0.054\end{array}$

Number of pre-index mental-health

related ER visits

$\begin{array}{llll}-0.068 & 0.078 & -0.87 & 0.383\end{array}$

Pre-index mental-health related

physician visits

$\begin{array}{llll}0.014 & 0.028 & 0.48 & 0.632\end{array}$

Number of pre-index mental-health

related hospitalizations

$\begin{array}{llll}0.022 & 0.039 & 0.56 & 0.575\end{array}$

Pre-index mental health-related

pharmacy cost

$0.305 \quad-0.060 \quad 0.9546$

0.028

Year of index prescription (ref: 1999)

- 2000

$-0.044$

0.103

$-0.43$

0.670

- 2001

0.016

0.121

0.13

0.898

Index prescription (ref: quetiapine)

Risperidone

$\begin{array}{llll}-0.002 & 0.137 & -0.02 & 0.986\end{array}$

Olanzapine

0.117

0.126

2.22

0.035

Typicals

$-0.859$

0.139

$-6.17$

$<.0001$

\section{Model fit statistics:}

Adjusted R-square $=25.70 \%, \mathbf{F}=9.91, \mathbf{p}<0.0001$ 
Table 33: Ordinary Least Squares (OLS) regression model for the impact of index antipsychotic on mental healthcare-related outpatient cost

\begin{tabular}{|c|c|c|c|c|}
\hline & Beta & S.E & $t$-statistic & $\begin{array}{c}\text { Significance } \\
(p)\end{array}$ \\
\hline \multicolumn{5}{|l|}{ Demographic characteristics } \\
\hline Age (in years) & 0.003 & 0.015 & 0.17 & 0.865 \\
\hline $\begin{array}{l}18 \text { years or above (ref: less } \\
\text { than } 18 \text { years) }\end{array}$ & -0.194 & 0.431 & -0.45 & 0.653 \\
\hline Males (ref: females) & 0.005 & 0.002 & 2.08 & 0.038 \\
\hline Whites (ref: non-whites) & 1.653 & 0.574 & 2.88 & 0.004 \\
\hline Metro (ref: non-metro) & -0.896 & 0.438 & -2.05 & 0.041 \\
\hline \multicolumn{5}{|l|}{ Prescribing physician type } \\
\hline Psychiatric prescriber & 0.001 & 0.000 & 2.29 & 0.022 \\
\hline \multicolumn{5}{|c|}{ Bipolar disorder subtype (ref: bipolar disorder I) } \\
\hline Bipolar disorder II & 0.524 & 0.462 & 1.13 & 0.258 \\
\hline Mixed & -0.929 & 0.481 & -2.89 & $<.0001$ \\
\hline \multicolumn{5}{|l|}{ Pre-index co-morbidities } \\
\hline Alcohol and substance abuse & 1.048 & 0.425 & 2.46 & 0.014 \\
\hline Schizophrenia & 0.747 & 0.543 & 1.37 & 0.170 \\
\hline Major depression & 0.101 & 0.452 & 0.22 & 0.823 \\
\hline Mild to Moderate depression & 0.785 & 0.535 & 1.47 & 0.143 \\
\hline Other mental comorbidities & -1.456 & 0.394 & -3.7 & 0.000 \\
\hline diabetes & -0.068 & 0.607 & -0.11 & 0.911 \\
\hline hyperlipedemia & -0.441 & 0.678 & -0.65 & 0.516 \\
\hline hypertension & -0.335 & 0.478 & -0.7 & 0.483 \\
\hline Charlson Comorbidity Index & -0.153 & 0.135 & -1.13 & 0.257 \\
\hline \multicolumn{5}{|c|}{ Pre-index period psychiatric medication use (days of supply) } \\
\hline Mood stabilizers & -0.001 & 0.001 & -0.84 & 0.399 \\
\hline Antipsychotics & -0.006 & 0.004 & -1.29 & 0.197 \\
\hline Antidepressants & 0.000 & 0.001 & -0.26 & 0.794 \\
\hline Benzodiazepines & 0.003 & 0.001 & 2.51 & 0.012 \\
\hline
\end{tabular}


Table 33: Ordinary Least Squares (OLS) regression model for the impact of index antipsychotic on mental healthcare-related outpatient cost (contd.)

\begin{tabular}{|c|c|c|c|c|}
\hline & Beta & S.E & t-statistic & $\begin{array}{c}\text { Significance } \\
(p)\end{array}$ \\
\hline \multicolumn{5}{|c|}{ Pre-index healthcare utilization and cost } \\
\hline $\begin{array}{l}\text { Number of psychotherapy } \\
\text { visits }\end{array}$ & -0.017 & 0.042 & -0.41 & 0.683 \\
\hline $\begin{array}{l}\text { Number of medication } \\
\text { management visits }\end{array}$ & 0.053 & 0.059 & 0.9 & 0.368 \\
\hline $\begin{array}{l}\text { Number of pre-index mental- } \\
\text { health related ER visits }\end{array}$ & -0.307 & 0.332 & -0.92 & 0.356 \\
\hline $\begin{array}{l}\text { Pre-index mental-health } \\
\text { related physician visits }\end{array}$ & 0.015 & 0.121 & 0.12 & 0.903 \\
\hline $\begin{array}{l}\text { Number of pre-index mental- } \\
\text { health related hospitalizations }\end{array}$ & 0.374 & 0.168 & 2.23 & 0.026 \\
\hline $\begin{array}{l}\text { Pre-index mental health- } \\
\text { related outpatient cost }\end{array}$ & 0.371 & 0.027 & 13.63 & $<.0001$ \\
\hline \multicolumn{5}{|c|}{ Year of index prescription (ref: 1999) } \\
\hline - 2000 & -0.323 & 0.442 & -0.73 & 0.466 \\
\hline - 2001 & -0.623 & 0.518 & -1.2 & 0.230 \\
\hline \multicolumn{5}{|c|}{ Index prescription (ref: quetiapine) } \\
\hline Risperidone & -0.806 & 0.584 & -1.38 & 0.168 \\
\hline Olanzapine & -0.629 & 0.537 & -1.17 & 0.241 \\
\hline Typicals & 0.075 & 0.597 & 2.95 & 0.003 \\
\hline
\end{tabular}

\section{Model fit statistics:}

Adjusted R-square $=38.22 \%, \mathbf{F}=16.93, \mathbf{p}<0.0001$ 


\section{Results for research objective 10}

For schizophrenia and bipolar patients, to compare the impact of quetiapine with risperidone, olanzapine, and typical antipsychotics on mental health-related healthcare hospitalizations.

$\underline{\text { Schizophrenia }}$

Results of univariate analysis of mental-health inpatient utilization are presented in table 34. Though there were significantly greater proportion of patients with pre-index mental health-related hospitalizations and number of inpatient visits in the quetiapine cohort as compared to the typical antipsychotic cohort, there were no significant differences in the proportion of patients having post-index hospitalization and the postindex length of stay between quetiapine and other atypical antipsychotics. Though the length of stay among pre-index hospitalized patients in olanzapine cohort was significantly longer compared to quetiapine cohort, there were no significant differences in the length of stay between quetiapine and other atypical antipsychotic cohorts. However, length of stay in the post-index period was significantly shorter for quetiapine $(9.9 \pm 12.6)$ as compared to typical antipsychotics $(13.4 \pm 11.0)$.

Multivariate analysis for the number of hospitalization visits

As number of hospitalization is count data, poisson or negative binomial model was used to compare the impact of index antipsychotic on number of mental healthrelated hospitalizations. Poisson regression assumes that the conditional mean of the outcome is equal to conditional variance. Poisson regression model carried out for estimating the number of hospitalizations was found to be overdispersed ie. the variance was greater than the conditional mean. This was due to high proportion of patients with 
zero count. Therefore, a negative binomial model was used for estimation of number of hospitalization as it relaxes the assumption that the conditional mean should be equal to the variance. The model statistics and the results are presented in table 35. The results show that there is no significant impact of receiving quetiapine as index antipsychotic compared to other index antipsychotics on number of mental health-related hospitalizations.

Time to the first-mental health-related hospitalization

Univariate Kaplan Meier analysis revealed that time to the first mental healthrelated hospitalization was not significantly different among the index cohorts as indicated by log rank statistics of $1.43(\mathrm{p}=0.696)$. The Kaplan Meier survival curves are presented in figure 4.

A multivariate Cox proportional model that controlled for confounders did not show any significant impact of using quetiapine as index antipsychotic on time to first mental health-related hospitalization (table 36).

\section{Bipolar Disorder}

Results of univariate analysis of mental-health inpatient utilization in bipolar disorder patients are presented in table 37. There were no significant differences between quetiapine and other antipsychotics in terms of post-index proportion of patients with mental health-related inpatient visits, number of inpatient visits and length of stay.

The results of the negative binomial model show that there is no significant impact of receiving quetiapine as index antipsychotic compared to other index antipsychotics on number of mental health-related hospitalizations (table 38). 
Table 34: Mental health-related hospitalization comparison (Quetiapine versus other antipsychotics) among schizophrenia patients

$\begin{array}{cccc}\text { Olanzapine } & \text { Risperidone } & \text { Quetiapine } & \text { Typical } \\ N=346 & N=201 & N=149 & N=303\end{array}$

\section{Patients hospitalized}

$\mathbf{N}(\%)$

$\begin{array}{ccccc} & 126 & 66 & 58^{\mathrm{T}} & 79^{\mathrm{Q}} \\ & (36.4 \%) & (32.8 \%) & (38.9 \%) & (26.1 \%) \\ \text { Pre-index } & & & & \\ \text { Post-index } & 107 & 52 & 46 & 87 \\ & (30.9 \%) & (25.9 \%) & (30.9 \%) & (28.7 \%)\end{array}$

Number of Inpatient Visits

\section{Mean $(+\underline{+}$ std $)$}

$\begin{array}{ccccc} & 0.6 & 0.5 & 0.5^{\mathrm{T}} & 0.4^{\mathrm{Q}} \\ \text { Pre-index } & ( \pm 1.1) & ( \pm 1.2) & ( \pm 0.8) & ( \pm 0.7)\end{array}$

$\begin{array}{ccccc}\text { Post-index } & 0.6 & 0.5 & 0.5 & 0.6 \\ ( \pm 1.2) & ( \pm 1.1) & ( \pm 1.1) & ( \pm 1.2)\end{array}$

\section{Length of Stay}

\section{Mean ( $(+\mathbf{s t d})$}

Pre-index

$$
13.4^{\mathrm{Q}}
$$

13.6

(+40.9)

10.2

$(+9.4)$
$8.9^{\mathrm{O}}$

$( \pm 7.8)$

$$
12.2
$$

(+13.6)
$9.9^{\mathrm{T}}$

(+12.6)
$13.4^{\mathrm{Q}}$

(+11.0)

\footnotetext{
${ }^{\mathrm{O}}$ Significant difference between quetiapine and olanzapine cohorts at $\mathrm{p}<0.05$

${ }^{\mathrm{R}}$ Significant difference between quetiapine and risperidone cohorts at $\mathrm{p}<0.05$

${ }^{\mathrm{T}}$ Significant difference between quetiapine and typicals cohorts at $\mathrm{p}<0.05$
} 
Table 35: Negative binomial model for the impact of index antipsychotic therapy of number of mental health-related hospitalization among schizophrenia patients

\begin{tabular}{|c|c|c|c|c|}
\hline & Beta & S.E & t-statistic & $\begin{array}{c}\text { Significance } \\
\text { (p) }\end{array}$ \\
\hline \multicolumn{5}{|l|}{ Demographic characteristics } \\
\hline Age (in years) & 0.004 & 0.006 & 0.590 & 0.442 \\
\hline Males (ref: females) & 0.005 & 0.007 & 0.540 & 0.464 \\
\hline Whites (ref: non-whites) & 0.610 & 0.234 & 6.780 & 0.009 \\
\hline Metro (ref: non-metro) & -0.131 & 0.153 & 0.740 & 0.390 \\
\hline \multicolumn{5}{|l|}{ Prescribing physician type } \\
\hline Psychiatric prescriber & 0.000 & 0.000 & 0.160 & 0.691 \\
\hline \multicolumn{5}{|c|}{ Schizophrenia subtype (ref: only schizophrenia) } \\
\hline Schizoaffective disorder & -0.501 & 0.164 & 9.370 & 0.002 \\
\hline $\begin{array}{l}\text { Both schizoaffective disorder } \\
\text { and schizophrenia }\end{array}$ & -0.695 & 0.176 & 15.640 & $<.0001$ \\
\hline \multicolumn{5}{|l|}{ Pre-index co-morbidities } \\
\hline Alcohol and substance abuse & 0.859 & 0.134 & 41.120 & $<.0001$ \\
\hline Bipolar disorder & 0.227 & 0.185 & 1.500 & 0.220 \\
\hline Major depression & 0.147 & 0.162 & 0.820 & 0.366 \\
\hline Mild to Moderate depression & 0.390 & 0.176 & 4.900 & 0.027 \\
\hline Other mental comorbidities & 0.173 & 0.146 & 1.400 & 0.236 \\
\hline diabetes & 0.435 & 0.201 & 4.700 & 0.030 \\
\hline hyperlipedemia & -0.229 & 0.251 & 0.830 & 0.362 \\
\hline hypertension & -0.062 & 0.175 & 0.120 & 0.724 \\
\hline Charlson Comorbidity Index & 0.169 & 0.051 & 11.160 & 0.001 \\
\hline \multicolumn{5}{|c|}{ Pre-index period psychiatric medication use (days of supply) } \\
\hline Mood stabilizers & 0.000 & 0.001 & 0.300 & 0.585 \\
\hline Antipsychotics & 0.001 & 0.002 & 0.280 & 0.597 \\
\hline Antidepressants & 0.001 & 0.000 & 1.810 & 0.178 \\
\hline Benzodiazepines & 0.001 & 0.001 & 0.880 & 0.348 \\
\hline
\end{tabular}


Table 35: Negative binomial model for the impact of index antipsychotic therapy of number of mental health-related hospitalization among schizophrenia patients (contd.)

\begin{tabular}{lcccc}
\hline & Beta & S.E & t-statistic & $\begin{array}{c}\text { Significance } \\
(\boldsymbol{p})\end{array}$ \\
$\begin{array}{l}\text { Pre-index healthcare utilization and cost } \\
\begin{array}{l}\text { Number of psychotherapy } \\
\text { visits }\end{array}\end{array}$ & -0.002 & 0.013 & 0.030 & 0.866 \\
$\begin{array}{l}\text { Number of medication } \\
\text { management visits }\end{array}$ & -0.066 & 0.024 & 7.390 & 0.007 \\
$\begin{array}{l}\text { Number of pre-index mental- } \\
\text { health related ER visits }\end{array}$ & -0.096 & 0.117 & 0.670 & 0.413 \\
$\begin{array}{l}\text { Pre-index mental-health } \\
\text { related physician visits }\end{array}$ & -0.049 & 0.043 & 1.270 & 0.261 \\
$\begin{array}{l}\text { Number of pre-index mental- } \\
\text { health related hospitalizations }\end{array}$ & 0.291 & 0.059 & 24.010 & $<.0001$ \\
$\begin{array}{l}\text { Year of index prescription (ref: } 1999) \\
\quad \text { - } 2000\end{array}$ & 0.203 & 0.166 & 1.490 & 0.222 \\
$\quad$ - 2001 & -0.170 & 0.205 & 0.690 & 0.406 \\
$\begin{array}{l}\text { Index prescription (ref: quetiapine) } \\
\text { Risperidone }\end{array}$ & -0.276 & 0.219 & 1.590 & 0.208 \\
$\begin{array}{l}\text { Olanzapine } \\
\text { Typicals }\end{array}$ & -0.307 & 0.194 & 2.510 & 0.113 \\
\hline
\end{tabular}

\section{Model fit statistics:}

-2 Log Likehood $=1008.08, \chi^{2}=220.39, p=0.0001$

Dispersion estimate $=1.36, \mathrm{p}=0.00$ 
Figure 4: Kaplan Meier Survival Curves for time to first mental health-related hospitalization among schizophrenia patients

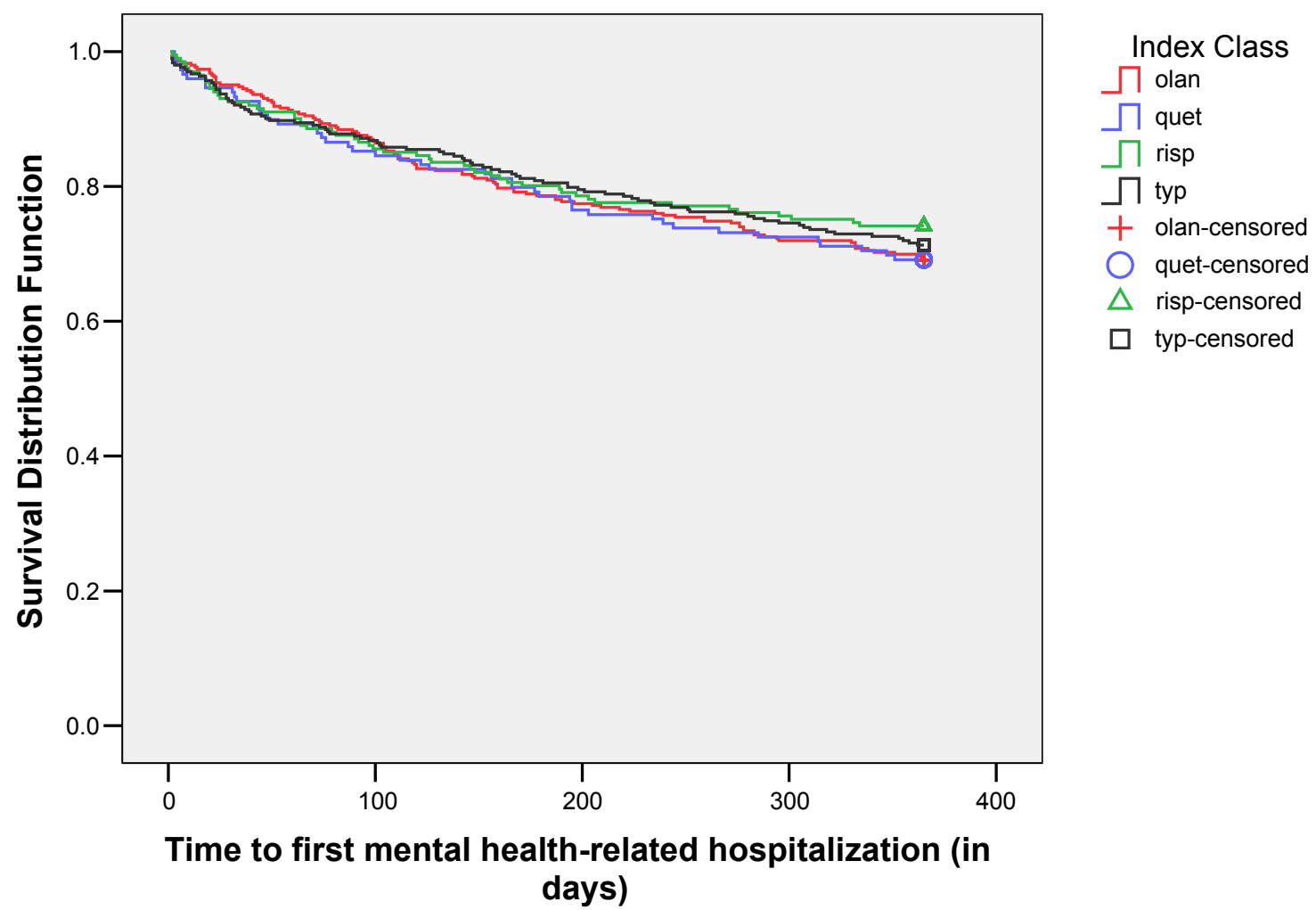

Overall Comparisons

\begin{tabular}{|l|r|r|r|}
\hline & Chi-Square & df & \multicolumn{1}{c|}{ Sig. } \\
\hline Log Rank (Mantel-Cox) & 1.439 & & 3 \\
\hline
\end{tabular}

Test of equality of survival distributions for the different levels of index class 
Table 36: Cox Proportional hazard model for the impact of index antipsychotic therapy on time to the first mental health-related hospitalization among schizophrenia patients (contd.)

\begin{tabular}{|c|c|c|c|c|c|c|}
\hline & \multirow[t]{2}{*}{ Beta } & \multirow[t]{2}{*}{$S E$} & \multirow[t]{2}{*}{ Sig. (p) } & \multirow[t]{2}{*}{$\begin{array}{l}\text { Hazards } \\
\text { Ratio }\end{array}$} & \multicolumn{2}{|c|}{$\begin{array}{l}\text { 95\% CI for hazards } \\
\text { ratio }\end{array}$} \\
\hline & & & & & $\begin{array}{c}\text { Lower } \\
\text { limit }\end{array}$ & $\begin{array}{c}\text { Upper } \\
\text { limit }\end{array}$ \\
\hline \multicolumn{7}{|c|}{ Demographic characteristics } \\
\hline Age (in years) & 0.003 & 0.007 & 0.727 & 1.003 & 0.988 & 1.017 \\
\hline Males (ref: females) & -0.461 & 0.161 & 0.004 & 0.631 & 0.461 & 0.864 \\
\hline $\begin{array}{l}\text { Whites (ref: non- } \\
\text { whites) }\end{array}$ & 0.488 & 0.305 & 0.110 & 1.628 & 0.896 & 2.959 \\
\hline $\begin{array}{l}\text { Metro (ref: non- } \\
\text { metro) }\end{array}$ & -0.279 & 0.186 & 0.134 & 0.757 & 0.526 & 1.089 \\
\hline \multicolumn{7}{|c|}{ Prescribing physician type } \\
\hline $\begin{array}{l}\text { Psychiatric } \\
\text { prescriber }\end{array}$ & -0.105 & 0.186 & 0.574 & 0.901 & 0.625 & 1.298 \\
\hline \multicolumn{7}{|c|}{ Schizophrenia subtype (ref: only schizophrenia) } \\
\hline $\begin{array}{l}\text { Schizoaffective } \\
\text { disorder }\end{array}$ & -0.392 & 0.195 & 0.045 & 0.675 & 0.461 & 0.990 \\
\hline $\begin{array}{l}\text { Both schizoaffective } \\
\text { disorder and } \\
\text { schizophrenia }\end{array}$ & -0.699 & 0.209 & 0.001 & 0.497 & 0.330 & 0.748 \\
\hline \multicolumn{7}{|c|}{ Pre-index co-morbidities } \\
\hline $\begin{array}{l}\text { Alcohol and } \\
\text { substance abuse }\end{array}$ & 0.658 & 0.163 & $<.0001$ & 1.931 & 1.403 & 2.657 \\
\hline Bipolar disorder & 0.357 & 0.218 & 0.101 & 1.429 & 0.933 & 2.190 \\
\hline Major depression & 0.170 & 0.191 & 0.373 & 1.185 & 0.815 & 1.724 \\
\hline $\begin{array}{l}\text { Mild to Moderate } \\
\text { depression }\end{array}$ & 0.241 & 0.195 & 0.216 & 1.273 & 0.869 & 1.866 \\
\hline $\begin{array}{l}\text { Other mental } \\
\text { comorbidities }\end{array}$ & 0.076 & 0.188 & 0.688 & 1.078 & 0.746 & 1.559 \\
\hline diabetes & 0.303 & 0.233 & 0.192 & 1.354 & 0.859 & 2.136 \\
\hline hyperlipedemia & -0.490 & 0.317 & 0.123 & 0.613 & 0.329 & 1.141 \\
\hline hypertension & -0.235 & 0.208 & 0.259 & 0.791 & 0.526 & 1.189 \\
\hline
\end{tabular}


Table 36: Cox Proportional hazard model for the impact of index antipsychotic therapy on time to the first mental health-related hospitalization among schizophrenia patients (contd.)

\begin{tabular}{|c|c|c|c|c|c|c|}
\hline & \multirow[t]{2}{*}{ Beta } & \multirow[t]{2}{*}{$S E$} & \multirow[t]{2}{*}{ Sig. (p) } & \multirow[t]{2}{*}{$\begin{array}{c}\text { Hazards } \\
\text { Ratio }\end{array}$} & \multicolumn{2}{|c|}{$\begin{array}{c}\text { 95\% CI for hazards } \\
\text { ratio }\end{array}$} \\
\hline & & & & & $\begin{array}{c}\text { Lower } \\
\text { limit }\end{array}$ & $\begin{array}{c}\text { Upper } \\
\text { limit }\end{array}$ \\
\hline \multicolumn{7}{|l|}{ Charlson } \\
\hline Comorbidity Index & 0.168 & 0.051 & 0.001 & 1.183 & 1.071 & 1.306 \\
\hline \multicolumn{7}{|c|}{ Pre-index period psychiatric medication use (days of supply) } \\
\hline Mood stabilizers & 0.000 & 0.001 & 0.959 & 1.000 & 0.999 & 1.001 \\
\hline Antipsychotics & -0.002 & 0.003 & 0.448 & 0.998 & 0.992 & 1.003 \\
\hline Antidepressants & 0.000 & 0.000 & 0.672 & 1.000 & 0.999 & 1.001 \\
\hline Benzodiazepines & 0.000 & 0.001 & 0.673 & 1.000 & 0.999 & 1.001 \\
\hline \multicolumn{7}{|c|}{ Pre-index healthcare utilization and cost } \\
\hline $\begin{array}{l}\text { Number of } \\
\text { psychotherapy visits }\end{array}$ & 0.004 & 0.012 & 0.738 & 1.004 & 0.981 & 1.027 \\
\hline $\begin{array}{l}\text { Number of } \\
\text { medication } \\
\text { management visits }\end{array}$ & -0.072 & 0.029 & 0.014 & 0.931 & 0.879 & 0.986 \\
\hline $\begin{array}{l}\text { Any pre-index } \\
\text { mental-health } \\
\text { related ER visits }\end{array}$ & -0.011 & 0.091 & 0.904 & 0.989 & 0.828 & 1.181 \\
\hline $\begin{array}{l}\text { Pre-index mental- } \\
\text { health related } \\
\text { physician visits }\end{array}$ & 0.022 & 0.042 & 0.588 & 1.023 & 0.943 & 1.109 \\
\hline $\begin{array}{l}\text { Any pre-index } \\
\text { mental-health } \\
\text { related } \\
\text { hospitalizations }\end{array}$ & 0.154 & 0.059 & 0.008 & 1.167 & 1.040 & 1.309 \\
\hline \multicolumn{7}{|c|}{ Year of index prescription (ref: 1999) } \\
\hline - 2000 & 0.131 & 0.186 & 0.480 & 1.140 & 0.792 & 1.641 \\
\hline - 2001 & -0.110 & 0.229 & 0.631 & 0.896 & 0.571 & 1.404 \\
\hline \multicolumn{7}{|c|}{ Index prescription (ref: quetiapine) } \\
\hline Risperidone & -0.273 & 0.251 & 0.277 & 0.761 & 0.465 & 1.245 \\
\hline Olanzapine & -0.160 & 0.205 & 0.436 & 0.852 & 0.570 & 1.274 \\
\hline Typicals & -0.250 & 0.235 & 0.288 & 0.779 & 0.491 & 1.235 \\
\hline
\end{tabular}


Model fit statistics:

-2 Log Likelihood $=2349.62, \chi^{2}=100.91, p<0.0001$ 
Table 37: Mental health-related hospitalization comparison (Quetiapine versus other antipsychotics) among bipolar disorder patients

\begin{tabular}{|c|c|c|c|c|}
\hline & $\begin{array}{c}\text { Olanzapine } \\
N=283\end{array}$ & $\begin{array}{c}\text { Risperidone } \\
\qquad=231\end{array}$ & $\begin{array}{c}\text { Quetiapine } \\
N=106\end{array}$ & $\begin{array}{c}\text { Typical } \\
N=205\end{array}$ \\
\hline \multicolumn{5}{|c|}{$\begin{array}{l}\text { Patients hospitalized } \\
\text { N }(\%)\end{array}$} \\
\hline Pre-index & $\begin{array}{c}102 \\
(36.0 \%)\end{array}$ & $\begin{array}{c}72 \\
(31.1 \%)\end{array}$ & $\begin{array}{c}39^{\mathrm{T}} \\
(36.8 \%)\end{array}$ & $\begin{array}{c}51^{Q} \\
(24.8 \%)\end{array}$ \\
\hline Post-index & $\begin{array}{c}84 \\
(29.7 \%)\end{array}$ & $\begin{array}{c}60 \\
(26.0 \%)\end{array}$ & $\begin{array}{c}30 \\
(28.3 \%)\end{array}$ & $\begin{array}{c}59 \\
(28.9 \%)\end{array}$ \\
\hline \multicolumn{5}{|c|}{$\begin{array}{l}\text { Number of Inpatient Visits } \\
\text { Mean (std) }\end{array}$} \\
\hline Pre-index & $\begin{array}{c}0.7 \\
( \pm 1.1)\end{array}$ & $\begin{array}{c}0.5 \\
( \pm 0.9)\end{array}$ & $\begin{array}{c}0.5 \\
( \pm 1.2)\end{array}$ & $\begin{array}{c}0.3 \\
( \pm 0.7)\end{array}$ \\
\hline Post-index & $\begin{array}{c}0.5 \\
( \pm 1.1)\end{array}$ & $\begin{array}{c}0.6 \\
( \pm 1.1)\end{array}$ & $\begin{array}{c}0.4 \\
( \pm 1.1)\end{array}$ & $\begin{array}{c}0.5 \\
( \pm 1.0)\end{array}$ \\
\hline \multicolumn{5}{|c|}{$\begin{array}{l}\text { Length of Stay } \\
\text { Mean }(+ \text { std })\end{array}$} \\
\hline Pre-index & $\begin{array}{c}11.8 \\
(+18.6)\end{array}$ & $\begin{array}{c}11.2 \\
(+16.2)\end{array}$ & $\begin{array}{c}8.6 \\
( \pm 6.2)\end{array}$ & $\begin{array}{c}10.9 \\
( \pm 12.8)\end{array}$ \\
\hline Post-index & $\begin{array}{c}10.0 \\
( \pm 13.3)\end{array}$ & $\begin{aligned} & 9.2 \\
&(+10.1)\end{aligned}$ & $\begin{array}{c}8.2 \\
(+12.0)\end{array}$ & $\begin{array}{c}10.4 \\
( \pm 10.2)\end{array}$ \\
\hline
\end{tabular}

${ }^{\mathrm{o}}$ Significant difference between quetiapine and olanzapine cohorts at $\mathrm{p}<0.05$

${ }^{\mathrm{R}}$ Significant difference between quetiapine and risperidone cohorts at $p<0.05$

${ }^{\mathrm{T}}$ Significant difference between quetiapine and typicals cohorts at $\mathrm{p}<0.05$ 
Table 38: Negative binomial model for the impact of index antipsychotic therapy of number of mental health-related hospitalization among bipolar disorder patients

\begin{tabular}{|c|c|c|c|c|}
\hline & Beta & S.E & t-statistic & $\begin{array}{c}\text { Significance } \\
(p)\end{array}$ \\
\hline \multicolumn{5}{|l|}{ Demographic characteristics } \\
\hline Age (in years) & 0.009 & 0.006 & 2.190 & 0.139 \\
\hline $\begin{array}{l}18 \text { years or above (ref: less } \\
\text { than } 18 \text { years) }\end{array}$ & 0.292 & 0.194 & 2.280 & 0.131 \\
\hline Males (ref: females) & 0.005 & 0.008 & 0.460 & 0.499 \\
\hline Whites (ref: non-whites) & 0.772 & 0.287 & 7.240 & 0.007 \\
\hline Metro (ref: non-metro) & -0.119 & 0.177 & 0.460 & 0.500 \\
\hline \multicolumn{5}{|l|}{ Prescribing physician type } \\
\hline Psychiatric prescriber & 0.000 & 0.000 & 0.080 & 0.775 \\
\hline \multicolumn{5}{|c|}{ Bipolar disorder subtype (ref: bipolar disorder I) } \\
\hline Bipolar disorder II & -0.477 & 0.188 & 6.450 & 0.011 \\
\hline Mixed & -0.732 & 0.191 & 14.620 & 0.000 \\
\hline \multicolumn{5}{|l|}{ Pre-index co-morbidities } \\
\hline Alcohol and substance abuse & 0.898 & 0.150 & 35.910 & $<.0001$ \\
\hline Bipolar disorder & 0.212 & 0.207 & 1.050 & 0.305 \\
\hline Major depression & 0.108 & 0.181 & 0.350 & 0.552 \\
\hline Mild to Moderate depression & 0.221 & 0.200 & 1.230 & 0.268 \\
\hline Other mental comorbidities & 0.189 & 0.162 & 1.360 & 0.243 \\
\hline diabetes & 0.357 & 0.215 & 2.760 & 0.097 \\
\hline hyperlipedemia & -0.209 & 0.267 & 0.620 & 0.433 \\
\hline hypertension & 0.008 & 0.187 & 0.000 & 0.966 \\
\hline Charlson Comorbidity Index & 0.171 & 0.051 & 11.120 & 0.001 \\
\hline \multicolumn{5}{|c|}{ Pre-index period psychiatric medication use (days of supply) } \\
\hline Mood stabilizers & 0.000 & 0.001 & 0.170 & 0.679 \\
\hline Antipsychotics & 0.001 & 0.002 & 0.150 & 0.698 \\
\hline Antidepressants & 0.000 & 0.001 & 0.530 & 0.465 \\
\hline Benzodiazepines & 0.001 & 0.001 & 1.450 & 0.228 \\
\hline
\end{tabular}


Table 38: Negative binomial model for the impact of index antipsychotic therapy of number of mental health-related hospitalization among bipolar disorder patients (contd.)

\begin{tabular}{|c|c|c|}
\hline Beta & S.E & t-statistic \\
\hline
\end{tabular}

\section{Pre-index healthcare utilization and cost}

Number of psychotherapy

visits

$$
0.009
$$

0.016

0.310

0.579

Number of medication

management visits

$-0.084$

0.029

8.360

0.004

Number of pre-index mental-

health related ER visits

$-0.084$

0.123

0.470

0.493

Pre-index mental-health

related physician visits

$-0.026$

0.047

0.300

0.585

Number of pre-index mental-

health related hospitalizations

0.263

0.063

17.690

$<.0001$

Year of index prescription (ref: 1999)
- 2000
0.306
0.176
3.010
0.083
- 2001
$-0.186$
0.226
0.680
0.410

Index prescription (ref: quetiapine)

$\begin{array}{lllll}\text { Risperidone } & -0.158 & 0.242 & 0.430 & 0.514 \\ \text { Olanzapine } & -0.371 & 0.228 & 2.650 & 0.104 \\ \text { Typicals } & -0.158 & 0.242 & 0.430 & 0.514\end{array}$

\section{Model fit statistics:}

-2 Log Likehood $=880.56, \chi^{2}=323.98, p<0.0001$

Dispersion estimate $=1.05, \mathrm{p}=0.00$ 
The univariate Kaplan Meier analysis revealed that time to the first mental healthrelated hospitalization was not significantly different between the index cohorts as indicated by log rank statistics of $0.577(\mathrm{p}=0.902)$. The Kaplan Meier survival curves are presented in figure 5 .

A multivariate Cox proportional model that controlled for confounders did not show any significant impact of using quetiapine as index antipsychotic on time to first mental health-related hospitalization (table 39).

\section{Results for research objective 11}

For schizophrenia patients, to compare the impact of quetiapine with risperidone, olanzapine, and typical antipsychotics on mental healthcare-related emergency room visits.

$\underline{\text { Schizophrenia }}$

Results of univariate analysis of mental-health-related ER utilization are presented in table 40 . Univariate analysis revealed that there were significantly greater proportion of patients with pre-index mental health related ER visits and significantly greater number of pre-index mental health related ER visits in the quetiapine cohort as compared to typical cohort. However, there were no significant differences in the postindex comparisons.

Multivariate analysis of number of mental health-related ER visit

If the proportion of zeroes is disproportionately large, negative binomial regression has to be replaced by zero inflated models. Zero inflated poisson models allows for proportion of zero values to be higher than poisson or negative binomial regression. As there were many patients with no mental health-related ER visit, zero 
inflated model was used for multivariate comparisons. The model statistics and results are presented in the table 41 . Vuong statistic is significant and positive supporting the use of zero inflated model. Patients initiated on quetiapine did not have any significant difference in mental health related ER visits compared to patients initiated on other index antipsychotics.

\section{Bipolar Disorder}

Results of univariate analysis of mental-health-related ER utilization are presented in table 42 . Univariate analysis reveals that there were significantly a greater proportion of patients with pre-index mental health related ER visits in the quetiapine cohort $(11.3 \%)$ as compared to the typical cohort (1.9\%). Also, there was a significantly greater proportion of patients with pre-index mental health related ER visits in the risperidone cohort (15.1\%) compared to quetiapine cohort (11.3\%). However, there were no significant differences in the post-index comparisons.

The multivariate zero inflated model also did not show any significant differences in the mental health related ER visits among patients initiated on Quetiapine as compared to patients initiated on other index antipsychotics (Table 43). 
Figure 5: Kaplan Meier Survival Curves for time to first mental health-related hospitalization among bipolar disorder patients

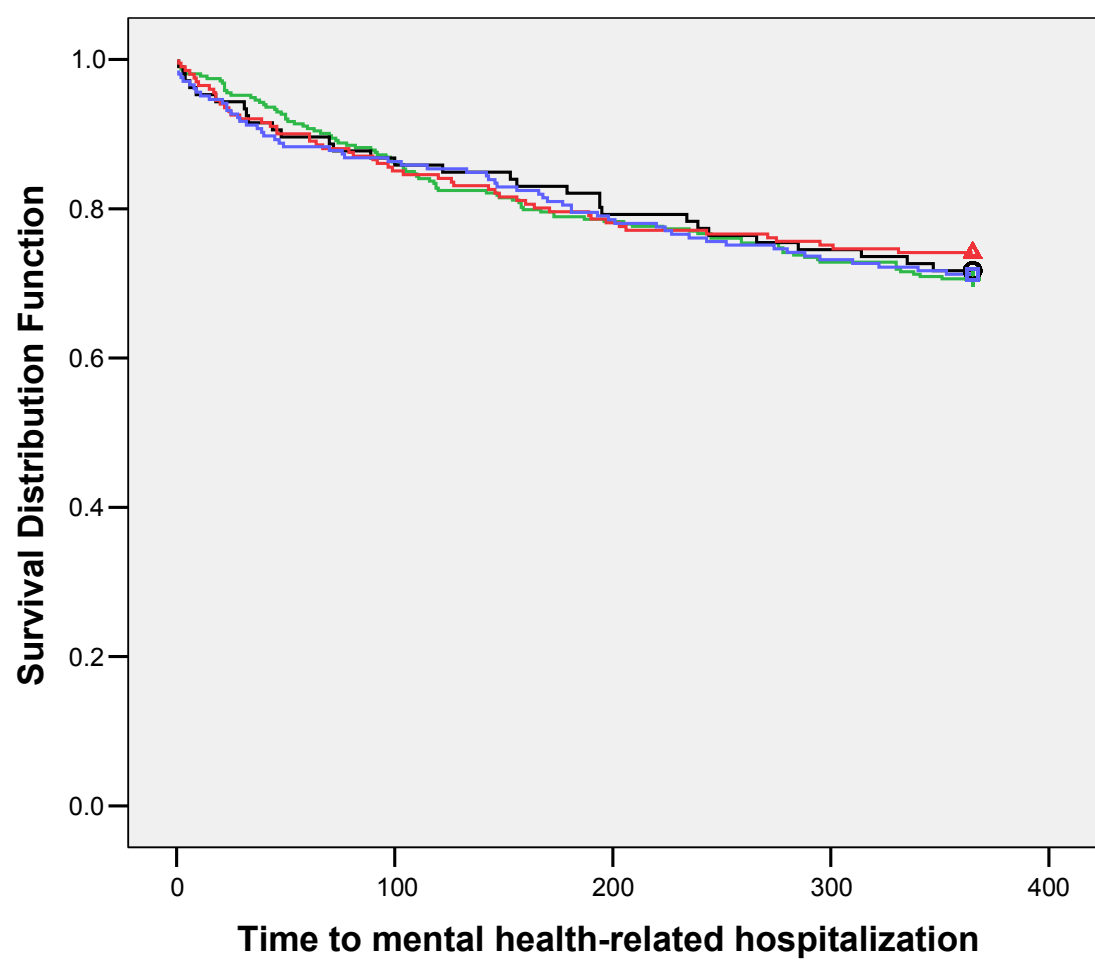

Index Class

$\sqcap$ olan

$\sqcap$ quet

$\sqcap$ risp

Пtyp

+ olan-censored

$\bigcirc$ quet-censored

$\triangle$ risp-censored

$\square$ typ-censored

Overall Comparisons

\begin{tabular}{|l|r|r|r|}
\hline & Chi-Square & df & \multicolumn{1}{c|}{ Sig. } \\
\hline Log Rank (Mantel-Cox) & .577 & 3 & .902 \\
\hline
\end{tabular}

Test of equality of survival distributions for the different levels of index class. 
Table 39: Cox Proportional hazard model for the impact of index antipsychotic therapy on time to the first mental health-related hospitalization among bipolar disorder patients

\begin{tabular}{|c|c|c|c|c|c|c|}
\hline & \multirow[t]{2}{*}{ Beta } & \multirow[t]{2}{*}{$S E$} & \multirow[t]{2}{*}{ Sig. (p) } & \multirow[t]{2}{*}{$\begin{array}{c}\text { Hazards } \\
\text { Ratio }\end{array}$} & \multicolumn{2}{|c|}{$\begin{array}{c}\text { 95\% CI for hazards } \\
\text { ratio }\end{array}$} \\
\hline & & & & & $\begin{array}{c}\text { Lower } \\
\text { limit }\end{array}$ & $\begin{array}{c}\text { Upper } \\
\text { limit }\end{array}$ \\
\hline \multicolumn{7}{|c|}{ Demographic characteristics } \\
\hline Age (in years) & 0.008 & 0.008 & 0.363 & 1.008 & 0.991 & 1.024 \\
\hline $\begin{array}{l}18 \text { years or above } \\
\text { (ref: less than } 18\end{array}$ & & & & & & \\
\hline years) & 0.039 & 0.234 & 0.866 & 1.040 & 0.658 & 1.646 \\
\hline Males (ref: females) & -0.490 & 0.179 & 0.006 & 0.613 & 0.431 & 0.871 \\
\hline $\begin{array}{l}\text { Whites (ref: non- } \\
\text { whites) }\end{array}$ & 0.722 & 0.374 & 0.054 & 2.058 & 0.988 & 4.287 \\
\hline $\begin{array}{l}\text { Metro (ref: non- } \\
\text { metro) }\end{array}$ & -0.284 & 0.218 & 0.192 & 0.753 & 0.492 & 1.153 \\
\hline \multicolumn{7}{|c|}{ Prescribing physician type } \\
\hline $\begin{array}{l}\text { Psychiatric } \\
\text { prescriber }\end{array}$ & -0.239 & 0.209 & 0.253 & 0.787 & 0.523 & 1.186 \\
\hline \multicolumn{7}{|c|}{ Bipolar disorder subtype (ref: Bipolar disorder I) } \\
\hline Bipolar disorder II & -0.487 & 0.231 & 0.035 & 0.614 & 0.390 & 0.967 \\
\hline Mixed & -0.926 & 0.238 & 0.000 & 0.396 & 0.248 & 0.632 \\
\hline \multicolumn{7}{|c|}{ Pre-index co-morbidities } \\
\hline $\begin{array}{l}\text { Alcohol and } \\
\text { substance abuse }\end{array}$ & 0.760 & 0.189 & $<.0001$ & 2.139 & 1.475 & 3.101 \\
\hline Bipolar disorder & 0.194 & 0.250 & 0.438 & 1.214 & 0.743 & 1.984 \\
\hline Major depression & 0.174 & 0.213 & 0.414 & 1.191 & 0.784 & 1.809 \\
\hline $\begin{array}{l}\text { Mild to Moderate } \\
\text { depression }\end{array}$ & 0.053 & 0.226 & 0.814 & 1.054 & 0.677 & 1.641 \\
\hline $\begin{array}{l}\text { Other mental } \\
\text { comorbidities }\end{array}$ & 0.246 & 0.209 & 0.240 & 1.279 & 0.849 & 1.927 \\
\hline Diabetes & 0.371 & 0.253 & 0.143 & 1.449 & 0.882 & 2.380 \\
\hline Hyperlipedemia & -0.288 & 0.336 & 0.392 & 0.750 & 0.388 & 1.449 \\
\hline Hypertension & -0.304 & 0.227 & 0.180 & 0.738 & 0.473 & 1.151 \\
\hline
\end{tabular}


Table 39: Cox Proportional hazard model for the impact of index antipsychotic therapy on time to the first mental health-related hospitalization among bipolar disorder patients (contd.)

\begin{tabular}{|c|c|c|c|c|c|c|}
\hline & \multirow[t]{2}{*}{ Beta } & \multirow[t]{2}{*}{$S E$} & \multirow[t]{2}{*}{ Sig. (p) } & \multirow[t]{2}{*}{$\begin{array}{l}\text { Hazards } \\
\text { Ratio }\end{array}$} & \multicolumn{2}{|c|}{$\begin{array}{c}\text { 95\% CI for hazards } \\
\text { ratio }\end{array}$} \\
\hline & & & & & $\begin{array}{c}\text { Lower } \\
\text { limit }\end{array}$ & $\begin{array}{c}\text { Upper } \\
\text { limit }\end{array}$ \\
\hline $\begin{array}{l}\text { Charlson } \\
\text { Comorbidity Index }\end{array}$ & 0.168 & 0.054 & 0.002 & 1.182 & 1.064 & 1.314 \\
\hline \multicolumn{7}{|c|}{ Pre-index period psychiatric medication use (days of supply) } \\
\hline Mood stabilizers & 0.000 & 0.001 & 0.691 & 1.000 & 0.999 & 1.002 \\
\hline Antipsychotics & -0.002 & 0.003 & 0.555 & 0.998 & 0.992 & 1.004 \\
\hline Antidepressants & 0.000 & 0.001 & 0.468 & 1.000 & 0.999 & 1.001 \\
\hline Benzodiazepines & 0.000 & 0.001 & 0.805 & 1.000 & 0.999 & 1.001 \\
\hline \multicolumn{7}{|c|}{ Pre-index healthcare utilization and cost } \\
\hline $\begin{array}{l}\text { Number of } \\
\text { psychotherapy visits }\end{array}$ & 0.020 & 0.022 & 0.364 & 1.020 & 0.977 & 1.064 \\
\hline $\begin{array}{l}\text { Number of } \\
\text { medication } \\
\text { management visits }\end{array}$ & -0.104 & 0.036 & 0.004 & 0.901 & 0.840 & 0.967 \\
\hline $\begin{array}{l}\text { Any pre-index } \\
\text { mental-health } \\
\text { related ER visits }\end{array}$ & -0.051 & 0.111 & 0.647 & 0.950 & 0.764 & 1.182 \\
\hline $\begin{array}{l}\text { Pre-index mental- } \\
\text { health related } \\
\text { physician visits }\end{array}$ & 0.069 & 0.049 & 0.158 & 1.071 & 0.974 & 1.179 \\
\hline $\begin{array}{l}\text { Any pre-index } \\
\text { mental-health } \\
\text { related } \\
\text { hospitalizations }\end{array}$ & 0.132 & 0.067 & 0.049 & 1.142 & 1.001 & 1.303 \\
\hline \multicolumn{7}{|c|}{ Year of index prescription (ref: 1999) } \\
\hline - 2000 & 0.119 & 0.211 & 0.571 & 1.127 & 0.746 & 1.702 \\
\hline - 2001 & -0.130 & 0.262 & 0.618 & 0.878 & 0.526 & 1.465 \\
\hline \multicolumn{7}{|c|}{ Index prescription (ref: quetiapine) } \\
\hline Risperidone & -0.312 & 0.289 & 0.279 & 0.732 & 0.416 & 1.288 \\
\hline Olanzapine & -0.227 & 0.248 & 0.361 & 0.797 & 0.490 & 1.297 \\
\hline
\end{tabular}


Table 39: Cox Proportional hazard model for the impact of index antipsychotic therapy on time to the first mental health-related hospitalization among bipolar disorder patients (contd.)

\begin{tabular}{lcccccc}
\hline & Beta & $S E$ & Sig. $(p)$ & $\begin{array}{c}\text { Hazards } \\
\text { Ratio }\end{array}$ & & \multicolumn{2}{c}{$\begin{array}{c}\text { 95\% CI for hazards } \\
\text { ratio }\end{array}$} \\
\cline { 5 - 7 } & & & & & $\begin{array}{c}\text { Lower } \\
\text { limit }\end{array}$ & $\begin{array}{c}\text { Upper } \\
\text { limit }\end{array}$ \\
\hline Typicals & -0.179 & 0.293 & 0.541 & 0.836 & 0.471 & 1.484 \\
\hline
\end{tabular}

\section{Model fit statistics:}

-2 Log Likelihood $=1822.14, \chi^{2}=94.26, p<0.0001$ 
Table 40: Mental health-related ER visits comparison (Quetiapine versus other antipsychotics) among schizophrenia patients

\begin{tabular}{|c|c|c|c|c|}
\hline & $\begin{array}{c}\text { Olanzapine } \\
N=346\end{array}$ & $\begin{array}{l}\text { Risperidone } \\
\quad N=201\end{array}$ & $\begin{array}{l}\text { Quetiapine } \\
N=149\end{array}$ & $\begin{array}{l}\text { Typical } \\
N=303\end{array}$ \\
\hline \multicolumn{5}{|c|}{$\begin{array}{l}\text { Patients with Mental-Health Related ER visits } \\
\text { N }(\%)\end{array}$} \\
\hline Pre-index & $\begin{array}{c}36 \\
(10.4 \%)\end{array}$ & $\begin{array}{c}24 \\
(11.9 \%)\end{array}$ & $\begin{array}{c}14^{\mathrm{T}} \\
(9.4 \%)\end{array}$ & $\begin{array}{c}10^{Q} \\
(3.3 \%)\end{array}$ \\
\hline Post-index & $\begin{array}{c}61 \\
(17.6 \%)\end{array}$ & $\begin{array}{c}31 \\
(15.4 \%)\end{array}$ & $\begin{array}{c}27 \\
(18.1 \%)\end{array}$ & $\begin{array}{c}47 \\
(15.5 \%)\end{array}$ \\
\hline \multicolumn{5}{|c|}{$\begin{array}{l}\text { Number of Mental -Health Related ER } \\
\text { Mean }( \pm \text { std })\end{array}$} \\
\hline Pre-index & $\begin{array}{c}0.14 \\
( \pm 0.49)\end{array}$ & $\begin{array}{c}0.21 \\
( \pm 0.81)\end{array}$ & $\begin{array}{c}0.12^{\mathrm{T}} \\
( \pm 0.41)\end{array}$ & $\begin{array}{c}0.04^{\mathrm{Q}} \\
( \pm 0.27)\end{array}$ \\
\hline Post-index & $\begin{array}{c}0.39 \\
(+1.63)\end{array}$ & $\begin{array}{c}0.23 \\
( \pm 0.67)\end{array}$ & $\begin{array}{c}0.32 \\
( \pm 0.84)\end{array}$ & $\begin{array}{c}0.26 \\
( \pm 0.82)\end{array}$ \\
\hline
\end{tabular}

\footnotetext{
Significant difference between quetiapine and olanzapine cohorts at $\mathrm{p}<0.05$

${ }^{\mathrm{R}}$ Significant difference between quetiapine and risperidone cohorts at $\mathrm{p}<0.05$

${ }^{\mathrm{T}}$ Significant difference between quetiapine and typicals cohorts at $\mathrm{p}<0.05$
} 
Table 41: Zero-inflated poisson regression model for the impact of index antipsychotic therapy of number of mental health-related ER visit among schizophrenia patients

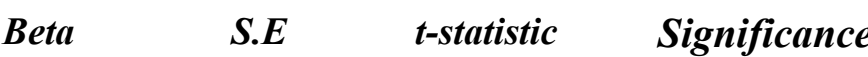

(p)

\section{Demographic characteristics}

Age (in years)

$0.010 \quad 0.006$

1.755

0.079

Males (ref: females)

$0.125 \quad 0.125$

1.005

0.315

Whites (ref: non-whites)

$0.989 \quad 0.299$

3.304

0.001

Metro (ref: non-metro)

0.107

0.138

0.775

0.438

Prescribing physician type

Psychiatric prescriber

$0.001 \quad 0.000$

4.673

0.000

Schizophrenia subtype (ref: only schizophrenia)

Schizoaffective disorder

$-0.690$

0.164

$-4.210$

0.000

Both schizoaffective disorder

and schizophrenia

$-0.745$

0.171

$-4.344$

0.000

\section{Pre-index co-morbidities}

Alcohol and substance abuse

0.668

0.127

5.265

0.000

Bipolar disorder

0.151

0.166

0.909

0.363

Major depression

0.455

0.141

3.232

0.001

Mild to Moderate depression

0.338

0.149

2.260

0.024

Other mental comorbidities

0.363

0.139

2.604

0.009

diabetes

$-0.153$

0.218

$-0.699$

0.485

hyperlipedemia

$-0.315$

0.282

$-1.119$

0.263

hypertension

$-0.144$

0.169

$-0.852$

0.394

Charlson Comorbidity Index

0.036

0.050

0.720

0.472

\section{Pre-index period psychiatric medication use}

\section{( days of supply)}

\begin{tabular}{lllll} 
Mood stabilizers & -0.001 & 0.001 & -1.862 & 0.063 \\
Antipsychotics & -0.014 & 0.004 & -3.410 & 0.001 \\
Antidepressants & 0.000 & 0.000 & -0.660 & 0.509 \\
Benzodiazepines & 0.000 & 0.001 & 0.613 & 0.540 \\
\hline
\end{tabular}


Table 41: Zero-inflated poisson regression model for the impact of index antipsychotic therapy of number of mental health-related ER visit among schizophrenia patients (contd.)

Beta S.E t-statistic Significance

(p)

Pre-index healthcare utilization and cost

Number of psychotherapy

visits

$-0.013$

0.012

$-1.126$

0.260

Number of medication

management visits

$-0.021$

0.022

$-0.950$

0.342

Number of pre-index mental-

health related ER visits

0.366

0.064

5.680

0.000

Pre-index mental-health

related physician visits

0.013

0.008

1.574

0.116

Number of pre-index mental-

health related hospitalizations

$-0.138$

0.065

$-2.136$

0.033

Year of index prescription (ref: 1999)

- 2000

$-0.006$

0.154

$-0.041$

0.967

- 2001

$-0.276$

0.189

$-1.459$

0.145

Index prescription (ref: quetiapine)

Risperidone

$-0.500$

0.225

$-2.218$

0.087

Olanzapine

0.039

0.176

0.224

0.823

Typicals

$-0.103$

0.196

$-0.524$

0.600

\section{Model fit statistics:}

Log Likelihood $=-702.84, \chi^{2}=331.27, p=0.0001$

Vuong statistic $=1.76, p=0.00$ 
Table 42: Mental health-related ER visits comparison (Quetiapine versus other antipsychotics) among bipolar disorder patients

$\begin{array}{cccc}\text { Olanzapine } & \text { Risperidone } & \text { Quetiapine } & \text { Typical } \\ N=283 & N=231 & N=106 & N=205\end{array}$

\section{Patients with Mental-Health Related ER visits}

N (\%)

Pre-index
32
$(11.3 \%)$
$35^{\mathrm{Q}}$
$(15.1 \%)$
$12^{\mathrm{T}}$
$(11.3 \%)$
$4^{\mathrm{Q}}$
$(1.9 \%)$

Post-index
53
$(18.7 \%)$

45

$(19.5 \%)$

19

34

$(17.9 \%)$

$(16.5 \%)$

Number of Mental -Health Related ER

Mean ( + std)

Pre-index

$\begin{array}{cccc}0.12 & 0.11 & 0.14 & 0.14 \\ ( \pm 0.51) & ( \pm 0.72) & ( \pm 0.52) & ( \pm 0.10)\end{array}$

Post-index
0.32
0.22
$( \pm 0.63)$
0.34
$( \pm 1.62)$
$( \pm 0.97)$

0.21

$( \pm 0.73)$

\footnotetext{
${ }^{\mathrm{O}}$ Significant difference between quetiapine and olanzapine cohorts at $\mathrm{p}<0.05$

${ }^{\mathrm{R}}$ Significant difference between quetiapine and risperidone cohorts at $\mathrm{p}<0.05$

${ }^{\mathrm{T}}$ Significant difference between quetiapine and typicals cohorts at $\mathrm{p}<0.05$
} 
Table 43: Zero-inflated poisson regression model for the impact of index antipsychotic therapy of number of mental health-related ER visit among bipolar disorder patients (contd.)

$\begin{array}{cccc}\text { Beta } & \text { S.E } & \text { t-statistic } & \text { Significance } \\ (p)\end{array}$

Demographic characteristics

Age (in years)

0.019

0.006

2.920

0.004

Males (ref: females)

0.298

0.196

1.525

0.127

Whites (ref: non-whites)

0.169

0.138

1.218

0.223

Metro (ref: non-metro)

1.029

0.333

3.089

0.002

Prescribing physician type

Psychiatric prescriber

0.006

0.158

0.036

0.972

Bipolar disorder subtype (ref: bipolar disorder I)

Bipolar disorder II

$0.001 \quad 0.000$

4.808

0.000

Mixed

$-0.756$

0.187

$-4.035$

0.000

\section{Pre-index co-morbidities}

Alcohol and substance abuse

$-0.962$

0.188

$-5.117$

0.000

Bipolar disorder

0.596

0.145

4.112

0.000

Major depression

0.208

0.182

1.146

0.252

Mild to Moderate depression

0.560

0.153

3.646

0.000

Other mental comorbidities

0.158

0.173

0.912

0.362

diabetes

0.565

0.156

3.625

0.000

hyperlipedemia

$-0.189$

0.231

$-0.817$

0.414

hypertension

$-0.104$

0.293

$-0.356$

0.722

Charlson Comorbidity Index

$-0.177$

0.180

$-0.984$

0.325

Pre-index period psychiatric medication use (days of supply)

Mood stabilizers

0.019

0.055

0.342

0.733

Antipsychotics

$-0.001$

0.001

$-1.997$

0.046

Antidepressants

$-0.012$

0.004

$-2.835$

0.005

Benzodiazepines

$-0.001$

0.000

$-1.242$

0.214

Pre-index healthcare utilization and cost

Number of psychotherapy

visits

0.001

0.001

0.886

0.376 
Table 43: Zero-inflated poisson regression model for the impact of index antipsychotic therapy of number of mental health-related ER visit among bipolar disorder patients (contd.)

$$
\text { Beta S.E t-statistic Significance }
$$

(p)

Number of medication

management visits

0.000

0.014

0.022

0.982

Number of pre-index mental-

health related ER visits

$-0.034$

0.025

$-1.348$

0.178

Pre-index mental-health

related physician visits

0.332

0.072

4.625

0.000

Number of pre-index mental-

health related hospitalizations

0.017

0.008

2.037

0.042

Year of index prescription (ref: 1999)
- 2000
$-0.138$
0.072
$-1.922$
0.055
- 2001
$-0.063$
0.171
$-0.371$
0.711

Index prescription (ref: quetiapine)

$\begin{array}{lllll}\text { Risperidone } & -0.193 & 0.205 & -0.940 & 0.347 \\ \text { Olanzapine } & -0.273 & 0.208 & -1.313 & 0.189 \\ \text { Typicals } & 0.627 & 0.244 & 2.566 & 0.110\end{array}$

\section{Model fit statistics:}

Log Likelihood $=-570.92, \chi^{2}=283.47, p=0.0001$

Vuong statistic $=1.59, \mathrm{p}=0.00$ 


\section{Results for research objective 12}

For schizophrenia and bipolar disorder patients, to compare the impact of quetiapine with risperidone, olanzapine, and typical antipsychotics on mental healthcare-related physician visits.

$\underline{\text { Schizophrenia }}$

Results of univariate analysis of mental-health-related outpatient utilization are presented in table 44. Patients in quetiapine cohort had a greater number of pre-index mental health-related physician office visits, psychotherapy sessions, and medication management visits than patients in other cohorts. Univariate analysis revealed the average number of post-index mental health-related physician office visits and medication management visits were significantly greater for quetiapine patients compared to other antipsychotics. The average number of psychotherapy sessions was significantly greater among risperidone patients $(1.25+7.83)$ as compared to quetiapine patients $(0.77+2.68)$.

Multivariate analysis to assess the impact of index antipsychotic was performed only for the mental health-related physician office visits. Results of negative binomial model revealed that mental health related physician office visit was not significantly different among patients initiated on quetiapine and other index antipsychotics (Table $45)$. 
Table 44: Physician office-visits, psychotherapy, and medication management psychotherapy sessions comparison (Quetiapine versus other antipsychotics) among schizophrenia patients

$\begin{array}{cccc}\text { Olanzapine } & \text { Risperidone } & \text { Quetiapine } & \text { Typical } \\ N=346 & N=201 & N=149 & N=303\end{array}$

Number of Mental Health-Related physician office visits

Mean $( \pm$ std)

Pre-index

$\begin{array}{cccc}0.57 & 0.33^{\mathrm{Q}} & 0.67^{\mathrm{T}} & 0.14^{\mathrm{Q}} \\ ( \pm 1.70) & ( \pm 1.14) & ( \pm 2.02) & ( \pm 0.84) \\ 0.86^{\mathrm{Q}} & 0.45^{\mathrm{Q}} & 1.06^{\mathrm{TR}} & 0.42^{\mathrm{Q}} \\ ( \pm 2.22) & ( \pm 1.17) & ( \pm 2.48) & ( \pm 1.27)\end{array}$

Number of Psychotherapy Sessions

Mean $( \pm \mathbf{s t d})$

Pre-index

0.76

$0.28^{\mathrm{Q}}$

$0.70^{\mathrm{T}}$

$0.26^{\mathrm{Q}}$

Post-index

(+6.31)

(+1.85)

$( \pm 3.98)$

(+3.24)

$1.25^{\mathrm{Q}}$

0.63

$0.77^{\mathrm{T}}$

$0.38^{\mathrm{Q}}$

(+7.83)

$( \pm 2.53)$

(+2.68)

$( \pm 2.92)$

Number of Medication Management Sessions

Mean $( \pm$ std)

Pre-index

$1.56^{\mathrm{Q}}$

$1.66^{\mathrm{Q}}$

2.44 OTR

$1.27^{\mathrm{Q}}$

$( \pm 2.79)$

$( \pm 2.88)$

$( \pm 4.15)$

$( \pm 2.61)$

Post-index

$\begin{array}{cc}3.49^{\mathrm{Q}} & 3.09^{\mathrm{Q}} \\ ( \pm 4.12) & ( \pm 4.12)\end{array}$

$4.29^{\text {OTR }}$

$0.38^{\mathrm{Q}}$

$(+4.25)$

$( \pm 2.92)$

\footnotetext{
${ }^{\mathrm{O}}$ Significant difference between quetiapine and olanzapine cohorts at $\mathrm{p}<0.05$

${ }^{\mathrm{R}}$ Significant difference between quetiapine and risperidone cohorts at $p<0.05$

${ }^{\mathrm{T}}$ Significant difference between quetiapine and typicals cohorts at $\mathrm{p}<0.05$
} 
Table 45: Negative binomial model for the impact of index antipsychotic therapy of number of mental health-related physician visit among schizophrenia patients

\begin{tabular}{|c|c|c|}
\hline Beta & S.E & t-statistic \\
\hline
\end{tabular}

\begin{tabular}{lllll}
\hline Demographic characteristics & & & & \\
Age (in years) & -0.005 & 0.008 & 0.310 & 0.577 \\
Males (ref: females) & 0.000 & 0.001 & 0.110 & 0.736 \\
Whites (ref: non-whites) & 0.365 & 0.342 & 1.140 & 0.286 \\
Metro (ref: non-metro) & 0.283 & 0.233 & 1.470 & 0.225
\end{tabular}

Prescribing physician type

$\begin{array}{lllll}\text { Psychiatric prescriber } & 0.000 & 0.000 & 2.120 & 0.146\end{array}$

Schizophrenia subtype (ref: only schizophrenia)

Schizoaffective disorder

$-0.226 \quad 0.262$

0.750

0.388

Both schizoaffective disorder

and schizophrenia

$-0.082$

0.271

0.090

0.763

Pre-index co-morbidities

Alcohol and substance abuse

0.155

0.226

0.470

0.491

Bipolar disorder

0.376

0.289

1.690

0.194

Major depression

0.675

0.240

7.900

0.005

Mild to Moderate depression

$-0.100$

0.287

0.120

0.729

Other mental comorbidities

$-0.007$

0.215

0.000

0.974

diabetes

0.303

0.334

0.820

0.364

hyperlipedemia

$-0.407$

0.390

1.090

0.296

hypertension

0.308

0.263

1.370

0.242

Charlson Comorbidity Index

$-0.166$

0.102

2.660

0.103

Pre-index period psychiatric medication use (days of supply)

Mood stabilizers

0.000

0.001

0.350

0.554

Antipsychotics

$-0.001$

0.003

0.040

0.849

Antidepressants

0.001

0.001

1.980

0.160

Benzodiazepines

0.001

0.001

0.650

0.419

Pre-index healthcare utilization and cost

Number of psychotherapy

visits

$-0.002$

0.026

0.000

0.944 
Table 45: Negative binomial model for the impact of index antipsychotic therapy of number of mental health-related physician visit among schizophrenia patients (contd.)

$\begin{array}{lll}\text { Beta } & \text { S.E } & \text {-statistic }\end{array}$

(p)

Number of medication

management visits

$-0.104 \quad 0.034$

9.330

Number of pre-index mental-

health related ER visits

0.135

0.185

0.530

0.467

Pre-index mental-health

related physician visits

0.325

0.073

0.022

0.098

0.050

0.824

Year of index prescription (ref: 1999)

- 2000

0.365

0.256

2.030

0.154

- 2001

0.230

0.306

0.570

0.451

Index prescription (ref: quetiapine)
$-0.147$

0.364

0.554
0.282

0.243

0.305

0.270

0.602

Typicals

\section{Model fit statistics:}

-2 Log Likelihood $=1074.08, \chi^{2}=179.09, p=0.0001$

Dispersion estimate $=1.11, \mathrm{p}=0.00$ 


\section{Bipolar Disorder}

Results of univariate analysis of mental-health-related outpatient utilization are presented in table 46 . The average number of post-index mental health-related physician office visits were significantly greater for quetiapine patients $(0.92+2.21)$ as compared to risperidone $(0.41+1.05)$ and typical antipsychotic $(0.48+1.43)$ patients. The average pre- and post-index numbers of psychotherapy sessions were significantly higher for quetiapine patients compared to typical antipsychotic cohort. The average numbers of medication management sessions were significantly higher for patients on quetiapine compared to patients on other antipsychotics.

Results of negative binomial model revealed that mental health related physician office visit was not significantly different among patients initiated on quetiapine and other index antipsychotics (Table 47).

\section{Results for research objective 13}

For schizophrenia patients, to compare the impact of quetiapine with risperidone, olanzapine, and typical antipsychotics on psychiatric medication utilization.

\section{$\underline{\text { Schizophrenia }}$}

Results of univariate analysis of mental-health-related pharmacy utilization are presented in table 48. Results of Mantel Hansel tests show that significantly greater proportions of patients in quetiapine cohort (39.6\%) were using mood stabilizers as compared to the olanzapine cohort (27.5\%), risperidone cohort (24.9\%) and typicals cohort (16.8\%) in the pre-index period. However, post-index comparisons showed significant differences only among quetiapine (55.0\%) and typical antipsychotic (32.3\%) cohort. 
Table 46: Physician office-visits, psychotherapy, and medication management psychotherapy sessions comparison (Quetiapine versus other antipsychotics) among bipolar disorder patients

\begin{tabular}{|c|c|c|c|c|}
\hline & $\begin{array}{c}\text { Olanzapine } \\
N=283\end{array}$ & $\begin{array}{l}\text { Risperidone } \\
\quad N=231\end{array}$ & $\begin{array}{l}\text { Quetiapine } \\
N=106\end{array}$ & $\begin{array}{l}\text { Typical } \\
N=205\end{array}$ \\
\hline \multicolumn{5}{|c|}{$\begin{array}{l}\text { Number of Mental Health-Related physician office visits } \\
\text { Mean }( \pm \text { std) }\end{array}$} \\
\hline Pre-index & $\begin{array}{c}0.55 \\
( \pm 1.63)\end{array}$ & $\begin{array}{c}0.30 \\
( \pm 1.03)\end{array}$ & $\begin{array}{c}0.60^{\mathrm{T}} \\
( \pm 1.90)\end{array}$ & $\begin{array}{l}0.20^{\mathrm{Q}} \\
( \pm 1.00)\end{array}$ \\
\hline Post-index & $\begin{array}{c}0.89 \\
(+2.29)\end{array}$ & $\begin{array}{l}0.41^{Q} \\
( \pm 1.05)\end{array}$ & $\begin{array}{l}0.92^{\mathrm{RT}} \\
( \pm 2.21)\end{array}$ & $\begin{array}{c}0.48^{\mathrm{Q}} \\
( \pm 1.43)\end{array}$ \\
\hline \multicolumn{5}{|c|}{$\begin{array}{l}\text { Number of Psychotherapy Sessions } \\
\text { Mean }( \pm \text { std })\end{array}$} \\
\hline Pre-index & $\begin{array}{c}0.57 \\
(+4.98)\end{array}$ & $\begin{array}{c}0.25 \\
( \pm 1.66)\end{array}$ & $\begin{array}{c}0.70^{\mathrm{T}} \\
( \pm 4.00)\end{array}$ & $\begin{array}{l}0.28^{\mathrm{Q}} \\
(+3.84)\end{array}$ \\
\hline Post-index & $\begin{array}{c}1.08 \\
( \pm 6.47)\end{array}$ & $\begin{array}{c}0.57 \\
( \pm 2.28)\end{array}$ & $\begin{array}{l}0.91^{\mathrm{T}} \\
( \pm 3.01)\end{array}$ & $\begin{array}{l}0.14^{\mathrm{Q}} \\
( \pm 1.50)\end{array}$ \\
\hline \multicolumn{5}{|c|}{$\begin{array}{l}\text { Number of Medication Management Sessions } \\
\text { Mean }( \pm \text { std })\end{array}$} \\
\hline Pre-index & $\begin{array}{c}1.57 \\
( \pm 2.86)\end{array}$ & $\begin{array}{c}1.50 \\
(+2.59)\end{array}$ & $\begin{array}{l}2.63^{\mathrm{T}} \\
( \pm 4.14)\end{array}$ & $\begin{array}{l}0.99^{\mathrm{Q}} \\
( \pm 2.47)\end{array}$ \\
\hline Post-index & $\begin{array}{l}3.57^{Q} \\
(+4.23)\end{array}$ & $\begin{array}{r}2.78^{Q} \\
(+3.76)\end{array}$ & $\begin{array}{l}4.72^{\text {ORT }} \\
( \pm 4.20)\end{array}$ & $\begin{array}{l}2.31 \mathrm{Q} \\
(+3.95)\end{array}$ \\
\hline
\end{tabular}

${ }^{\mathrm{O}}$ Significant difference between quetiapine and olanzapine cohorts at $\mathrm{p}<0.05$

${ }^{\mathrm{R}}$ Significant difference between quetiapine and risperidone cohorts at $\mathrm{p}<0.05$

${ }^{\mathrm{T}}$ Significant difference between quetiapine and typicals cohorts at $\mathrm{p}<0.05$ 
Table 47: Negative binomial model for the impact of index antipsychotic therapy of number of mental health-related physician visit among bipolar disorder patients

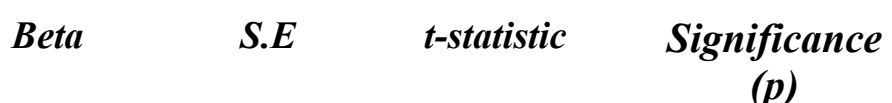

Demographic characteristics

$\begin{array}{lllll}\text { Age (in years) } & -0.006 & 0.009 & -0.500 & 0.479 \\ \begin{array}{l}\text { 18 years or above (ref: less } \\ \text { than 18 years) }\end{array} & -0.404 & 0.251 & -2.590 & 0.107 \\ \text { Males (ref: females) } & 0.000 & 0.001 & 0.000 & 0.969 \\ \text { Whites (ref: non-whites) } & 0.434 & 0.388 & 1.250 & 0.264 \\ \text { Metro (ref: non-metro) } & 0.195 & 0.262 & 0.550 & 0.457 \\ \begin{array}{l}\text { Prescribing physician type } \\ \text { Psychiatric prescriber }\end{array} & 0.000 & 0.000 & 1.770 & 0.183\end{array}$

Bipolar disorder subtype (ref: Bipolar disorder I)

Bipolar disorder II

$0.279 \quad 0.291$

0.920

0.339

Mixed

$-0.083$

0.296

$-0.080$

0.780

Pre-index co-morbidities

Alcohol and substance abuse

$\begin{array}{llll}0.195 & 0.253 & 0.590 & 0.441 \\ 0.274 & 0.320 & 0.730 & 0.392 \\ 0.711 & 0.262 & 7.370 & 0.007 \\ -0.181 & 0.316 & -0.330 & 0.568 \\ 0.116 & 0.232 & 0.250 & 0.618 \\ 0.269 & 0.366 & 0.540 & 0.463 \\ -0.269 & 0.410 & -0.430 & 0.511 \\ 0.324 & 0.284 & 1.300 & 0.254 \\ -0.174 & 0.105 & -2.740 & 0.098\end{array}$

Bipolar disorder

Major depression

Mild to Moderate depression

Other mental comorbidities

diabetes

$-0.174$

0.105

0.098

Pre-index period psychiatric medication use

( days of supply)

$\begin{array}{lllll}\text { Mood stabilizers } & 0.000 & 0.001 & 0.150 & 0.695 \\ \text { Antipsychotics } & -0.003 & 0.003 & -1.240 & 0.265 \\ \text { Antidepressants } & 0.001 & 0.001 & 0.580 & 0.448\end{array}$


Table 47: Negative binomial model for the impact of index antipsychotic therapy of number of mental health-related physician visit among bipolar disorder patients (contd.)

$$
\text { Beta S.E t-statistic Significance }
$$

(p)

Benzodiazepines

0.001

0.001

0.620

0.432

\section{Pre-index healthcare utilization and cost}

Number of psychotherapy

visits

0.000

0.030

0.000

0.990

Number of medication

management visits

$-0.096$

0.037

$-6.660$

0.010

Number of pre-index mental-

health related ER visits

0.109

0.200

0.300

0.585

Pre-index mental-health

related physician visits

0.330

0.075

19.530

$<.0001$

Number of pre-index mental-

health related hospitalizations

0.046

0.100

0.210

0.643

Year of index prescription (ref: 1999)
- 2000
0.331
0.273
1.470
0.225
- 2001
0.224
0.329
0.460
0.496

Index prescription (ref: quetiapine)

$\begin{array}{lllll}\text { Risperidone } & 0.564 & 0.357 & 2.490 & 0.115 \\ \text { Olanzapine } & 0.226 & 0.270 & 0.700 & 0.402 \\ \text { Typicals } & -0.067 & 0.290 & -0.050 & 0.818\end{array}$

\section{Model fit statistics:}

-2 Log Likelihood $=1059.08, \chi^{2}=129.05, p=0.0001$

Dispersion estimate $=1.01, \mathrm{p}=0.00$ 
Table 48: Psychiatric medication use comparison (Quetiapine versus other antipsychotics) among schizophrenia patients

\begin{tabular}{|c|c|c|c|c|}
\hline & $\begin{array}{c}\text { Olanzapine } \\
N=346\end{array}$ & $\begin{array}{c}\text { Risperidone } \\
\quad N=201\end{array}$ & $\begin{array}{c}\text { Quetiapine } \\
N=149\end{array}$ & $\begin{array}{l}\text { Typical } \\
N=303\end{array}$ \\
\hline \multicolumn{5}{|c|}{ Mood Stabilizers N (\%) } \\
\hline Pre-index & $\begin{array}{c}95^{\mathrm{Q}} \\
(27.5 \%)\end{array}$ & $\begin{array}{c}50^{\mathrm{Q}} \\
(24.9 \%)\end{array}$ & $\begin{array}{l}59^{\mathrm{ORT}} \\
(39.6 \%)\end{array}$ & $\begin{array}{c}51^{Q} \\
(16.8 \%)\end{array}$ \\
\hline Post-index & $\begin{array}{c}172 \\
(49.7 \%)\end{array}$ & $\begin{array}{c}98 \\
(48.8 \%)\end{array}$ & $\begin{array}{c}82^{\mathrm{T}} \\
(55.0 \%)\end{array}$ & $\begin{array}{c}98^{Q} \\
(32.3 \%)\end{array}$ \\
\hline \multicolumn{5}{|c|}{ Antidepressants N (\%) } \\
\hline Pre-index & $\begin{array}{c}159^{Q} \\
(45.9 \%)\end{array}$ & $\begin{array}{c}83^{Q} \\
(41.3 \%)\end{array}$ & $\begin{array}{c}92^{\text {ORT }} \\
(61.7 \%)\end{array}$ & $\begin{array}{c}93^{Q} \\
(30.7 \%)\end{array}$ \\
\hline Post-index & $\begin{array}{c}265 \\
(76.6 \%)\end{array}$ & $\begin{array}{c}140 \\
(69.6 \%)\end{array}$ & $\begin{array}{c}113^{\mathrm{T}} \\
(75.8 \%)\end{array}$ & $\begin{array}{c}164 \mathrm{Q} \\
(54.1 \%)\end{array}$ \\
\hline \multicolumn{5}{|c|}{ Anxiolytics/Hypnotics/Sedatives N (\%) } \\
\hline Pre-index & $\begin{array}{c}52 \\
(15.0 \%)\end{array}$ & $\begin{array}{c}22 \\
(10.9 \%)\end{array}$ & $\begin{array}{c}23^{\mathrm{T}} \\
(15.4 \%)\end{array}$ & $\begin{array}{c}20 \mathrm{Q} \\
(6.6 \%)\end{array}$ \\
\hline Post-index & $\begin{array}{c}67 \\
(19.3 \%)\end{array}$ & $\begin{array}{c}32 \\
(15.9 \%)\end{array}$ & $\begin{array}{c}29 \\
(19.5 \%)\end{array}$ & $\begin{array}{c}46 \\
(15.2 \%)\end{array}$ \\
\hline \multicolumn{5}{|c|}{ Antiparkinsons N (\%) } \\
\hline Pre-index & $\begin{array}{c}10 \\
(2.9 \%)\end{array}$ & $\begin{array}{c}3 \\
(1.5 \%)\end{array}$ & $\begin{array}{c}5 \\
(3.3 \%)\end{array}$ & $\begin{array}{c}17 \\
(5.6 \%)\end{array}$ \\
\hline Post-index & $\begin{array}{c}25 \\
(7.2 \%)\end{array}$ & $\begin{array}{c}13 \\
(6.5 \%)\end{array}$ & $\begin{array}{c}12^{\mathrm{T}} \\
(8.0 \%)\end{array}$ & $\begin{array}{c}66^{Q} \\
(21.8 \%)\end{array}$ \\
\hline
\end{tabular}

\footnotetext{
${ }^{\mathrm{O}}$ Significant difference between quetiapine and olanzapine cohorts at $\mathrm{p}<0.05$

${ }^{\mathrm{R}}$ Significant difference between quetiapine and risperidone cohorts at $\mathrm{p}<0.05$

${ }^{\mathrm{T}}$ Significant difference between quetiapine and typicals cohorts at $\mathrm{p}<0.05$
} 
Table 48: Psychiatric medication use comparison (Quetiapine versus other antipsychotics) among schizophrenia patients (contd.)

\begin{tabular}{|c|c|c|c|c|}
\hline & $\begin{array}{c}\text { Olanzapine } \\
N=346\end{array}$ & $\begin{array}{c}\text { Risperidone } \\
N=201\end{array}$ & $\begin{array}{c}\text { Quetiapine } \\
N=149\end{array}$ & $\begin{array}{c}\text { Typical } \\
N=303\end{array}$ \\
\hline \multicolumn{5}{|c|}{ Anticholinergics N (\%) } \\
\hline Pre-index & $\begin{array}{c}5 \\
(1.4 \%)\end{array}$ & $\begin{array}{c}2 \\
(1.0 \%)\end{array}$ & $\begin{array}{c}6 \\
(4.0 \%)\end{array}$ & $\begin{array}{c}1 \\
(0.3 \%)\end{array}$ \\
\hline Post-index & $\begin{array}{c}8 \\
(2.3 \%)\end{array}$ & $\begin{array}{c}6 \\
(2.9 \%)\end{array}$ & $\begin{array}{c}2 \\
(1.3 \%)\end{array}$ & $\begin{array}{c}4 \\
(1.3 \%)\end{array}$ \\
\hline \multicolumn{5}{|c|}{ Clozapine N (\%) } \\
\hline Pre-index & $\begin{array}{c}1 \\
(0.3 \%)\end{array}$ & $\begin{array}{c}0 \\
(0.0)\end{array}$ & $\begin{array}{c}1 \\
(0.7 \%)\end{array}$ & $\begin{array}{c}1 \\
(0.3 \%)\end{array}$ \\
\hline Post-index & $\begin{array}{c}5 \\
(1.4 \%)\end{array}$ & $\begin{array}{c}0 \\
(0.0)\end{array}$ & $\begin{array}{c}0 \\
(0.0)\end{array}$ & $\begin{array}{c}5 \\
(1.6 \%)\end{array}$ \\
\hline \multicolumn{5}{|c|}{ Benzodiazepine N (\%) } \\
\hline Pre-index & $\begin{array}{c}104 \\
(30.0 \%)\end{array}$ & $\begin{array}{c}59^{Q} \\
(29.3 \%)\end{array}$ & $\begin{array}{c}70^{\mathrm{RT}} \\
(46.9 \%)\end{array}$ & $\begin{array}{c}65^{\mathrm{Q}} \\
(21.4 \%)\end{array}$ \\
\hline Post-index & $\begin{array}{c}145^{\mathrm{Q}} \\
(41.9 \%)\end{array}$ & $\begin{array}{c}82^{Q} \\
(40.8 \%)\end{array}$ & $\begin{array}{l}79 \text { ORT } \\
(53.0 \%)\end{array}$ & $\begin{array}{c}123^{Q} \\
(40.6 \%)\end{array}$ \\
\hline
\end{tabular}

${ }^{\mathrm{O}}$ Significant difference between quetiapine and olanzapine cohorts at $\mathrm{p}<0.05$

${ }^{\mathrm{R}}$ Significant difference between quetiapine and risperidone cohorts at $\mathrm{p}<0.05$

${ }^{\mathrm{T}}$ Significant difference between quetiapine and typicals cohorts at $\mathrm{p}<0.05$ 
Though significantly greater proportions of patients in the quetiapine cohort (61.7\%) were using antidepressants compared to the olanzapine cohort (45.9\%), risperidone cohort (41.3\%) and typicals cohort (30.7\%) in the pre-index period, proportions of patients on antidepressants were almost similar among the atypical cohort in the post-index index. The quetiapine cohort did have greater proportions of patients (75.8\%) on antidepressants in the post-index period than typical antipsychotics (54.1\%).

Though there was no significant difference in proportions of patients on antiparkinsons drugs in the pre-index period between quetiapine and other antipsychotics, there were significantly greater proportion of patients on antiparkinson drugs among typical antipsychotic cohort (21.8\%) compared to quetiapine cohort (8.0\%) in the post-index period.

Proportions of patients with benzodiazepine use were greater in quetiapine cohort as compared to other antipsychotics in both pre-index and post-index periods. There were no significant differences in the proportion of patients using anxiolytics/ hypnotics/ sedatives in the pre-index and post-index periods. As the sample size of patients using clozapine was very small, statistical tests could not be carried out.

\section{Bipolar Disorder}

Results of univariate analysis of mental-health-related pharmacy utilization are presented in table 49. Results of Mantel Hansel tests show that there was no significant difference in the proportion of patients on mood stabilizers in the pre-index and postindex period between quetiapine and other antipsychotics. There were significantly a greater proportion of patients on antidepressants in the quetiapine cohort as compared to the other antipsychotics in the pre-index period. There were significantly a greater 
proportion of patients on antidepressants in the quetiapine cohort (81.1\%) than in typical antipsychotic cohort (51.7\%) in the post-index period. There were significantly greater proportions of patients on anxiolytics/ hypnotics/ sedatives in the quetiapine cohort during the pre-index period as compared to the other cohorsts. However, the proportions of patients on anxiolytics/ hypnotics/ sedatives were greater in the risperidone cohort (24.7\%) than quetiapine cohort (20.7\%) in the post-index period. The proportions of patients on anxiolytics/ hypnotics/ sedatives were greater in the quetiapine cohort than in the typical antipsychotic cohort (18.0\%).

There were significantly greater proportions of patients on antiparkinsons drugs in the typical antipsychotic cohort (19.0\%) than in the quetiapine cohort (5.6\%). There were a significantly greater proportion of patients $(48.1 \%)$ on benzodiazepines in the quetiapine cohort as compared to the other antipsychotics in the pre-index period. The post-index comparisons show significantly greater proportions of patients on benzodiazepines in the quetiapine cohort (48.1\%) compared to olanzapine (43.1\%) and typical antipsychotic cohorts (44.3\%). 
Table 49: Psychiatric medication use comparison (Quetiapine versus other antipsychotics) among bipolar disorder patients

\begin{tabular}{|c|c|c|c|c|}
\hline & $\begin{array}{c}\text { Olanzapine } \\
N=283\end{array}$ & $\begin{array}{c}\text { Risperidone } \\
\quad N=231\end{array}$ & $\begin{array}{l}\text { Quetiapine } \\
N=106\end{array}$ & $\begin{array}{r}\text { Typical } \\
N=205\end{array}$ \\
\hline \multicolumn{5}{|c|}{$\begin{array}{l}\text { Mood Stabilizers } \\
\text { N (\%) }\end{array}$} \\
\hline Pre-index & $\begin{array}{c}146 \\
(51.6 \%)\end{array}$ & $\begin{array}{c}101 \\
(43.7 \%)\end{array}$ & $\begin{array}{c}56 \\
(52.8 \%)\end{array}$ & $\begin{array}{c}102 \\
(49.7 \%)\end{array}$ \\
\hline Post-index & $\begin{array}{c}153 \\
(54.1 \%)\end{array}$ & $\begin{array}{c}98 \\
(42.4 \%)\end{array}$ & $\begin{array}{c}55 \\
(51.8 \%)\end{array}$ & $\begin{array}{c}112 \\
(54.6 \%)\end{array}$ \\
\hline \multicolumn{5}{|c|}{$\begin{array}{l}\text { Antidepressants } \\
\mathbf{N}(\%)\end{array}$} \\
\hline Pre-index & $\begin{array}{c}146^{\mathrm{Q}} \\
(51.6 \%)\end{array}$ & $\begin{array}{c}89^{Q} \\
(38.5 \%)\end{array}$ & $\begin{array}{c}68^{\mathrm{ORT}} \\
(64.1 \%)\end{array}$ & $\begin{array}{c}70^{\mathrm{Q}} \\
(34.1 \%)\end{array}$ \\
\hline Post-index & $\begin{array}{c}241 \\
(85.2 \%)\end{array}$ & $\begin{array}{c}180 \\
(77.9 \%)\end{array}$ & $\begin{array}{c}86^{\mathrm{T}} \\
(81.1 \%)\end{array}$ & $\begin{array}{c}106^{\mathrm{Q}} \\
(51.7 \%)\end{array}$ \\
\hline \multicolumn{5}{|c|}{$\begin{array}{l}\text { Anxiolytics/Hypnotics/Sedatives } \\
\text { N (\%) }\end{array}$} \\
\hline Pre-index & $\begin{array}{c}47^{\mathrm{Q}} \\
(16.6 \%)\end{array}$ & $\begin{array}{c}34^{\mathrm{Q}} \\
(14.7 \%)\end{array}$ & $\begin{array}{c}20^{\text {ORT }} \\
(18.8 \%)\end{array}$ & $\begin{array}{c}19^{Q} \\
(9.2 \%)\end{array}$ \\
\hline Post-index & $\begin{array}{c}62 \\
(21.9 \%)\end{array}$ & $\begin{array}{c}57^{\mathrm{Q}} \\
(24.7 \%)\end{array}$ & $\begin{array}{c}22^{\mathrm{RT}} \\
(20.7 \%)\end{array}$ & $\begin{array}{c}37^{\mathrm{Q}} \\
(18.0 \%)\end{array}$ \\
\hline \multicolumn{5}{|c|}{$\begin{array}{l}\text { Antiparkinsons } \\
\text { N (\%) }\end{array}$} \\
\hline Pre-index & $\begin{array}{c}10 \\
(3.5 \%)\end{array}$ & $\begin{array}{c}10 \\
(4.3 \%)\end{array}$ & $\begin{array}{c}4 \\
(3.7 \%)\end{array}$ & $\begin{array}{c}7 \\
(3.4 \%)\end{array}$ \\
\hline Post-index & $\begin{array}{c}24 \\
(8.4 \%)\end{array}$ & $\begin{array}{c}19 \\
(8.2 \%)\end{array}$ & $\begin{array}{c}6^{\mathrm{T}} \\
(5.6 \%)\end{array}$ & $\begin{array}{c}39^{Q} \\
(19.0 \%)\end{array}$ \\
\hline
\end{tabular}


Table 49: Psychiatric medication use comparison (Quetiapine versus other antipsychotics) among bipolar disorder patients (contd.)

\begin{tabular}{|c|c|c|c|c|}
\hline & $\begin{array}{c}\text { Olanzapine } \\
N=283\end{array}$ & $\begin{array}{l}\text { Risperidone } \\
\quad N=201\end{array}$ & $\begin{array}{l}\text { Quetiapine } \\
N=106\end{array}$ & $\begin{array}{c}\text { Typical } \\
N=205\end{array}$ \\
\hline \multicolumn{5}{|c|}{$\begin{array}{l}\text { Anticholinergics } \\
\text { N (\%) }\end{array}$} \\
\hline Pre-index & $\begin{array}{c}7 \\
(2.4 \%)\end{array}$ & $\begin{array}{c}5 \\
(2.4 \%)\end{array}$ & $\begin{array}{c}4 \\
(3.7 \%)\end{array}$ & $\begin{array}{c}2 \\
(0.9 \%)\end{array}$ \\
\hline Post-index & $\begin{array}{c}8 \\
(2.8 \%)\end{array}$ & $\begin{array}{c}4 \\
(1.7 \%)\end{array}$ & $\begin{array}{c}2 \\
(1.8 \%)\end{array}$ & $\begin{array}{c}8 \\
(3.9 \%)\end{array}$ \\
\hline \multicolumn{5}{|l|}{$\begin{array}{l}\text { Clozapine } \\
\text { N (\%) }\end{array}$} \\
\hline Pre-index & $\begin{array}{c}1 \\
(0.3 \%)\end{array}$ & $\begin{array}{c}0 \\
(0.0 \%)\end{array}$ & $\begin{array}{c}0 \\
(0.0 \%)\end{array}$ & $\begin{array}{c}1 \\
(0.3 \%)\end{array}$ \\
\hline Post-index & $\begin{array}{c}0 \\
(0.0 \%)\end{array}$ & $\begin{array}{c}0 \\
(0.0 \%)\end{array}$ & $\begin{array}{c}0 \\
(0.0 \%)\end{array}$ & $\begin{array}{c}2 \\
(0.9 \%)\end{array}$ \\
\hline \multicolumn{5}{|c|}{$\begin{array}{l}\text { Benzodiazepine } \\
\text { N (\%) }\end{array}$} \\
\hline Pre-index & $\begin{array}{c}94^{\mathrm{Q}} \\
(33.2 \%)\end{array}$ & $\begin{array}{c}92^{Q} \\
(39.8 \%)\end{array}$ & $\begin{array}{l}51^{\text {ORT }} \\
(48.1 \%)\end{array}$ & $\begin{array}{c}53^{Q} \\
(25.8 \%)\end{array}$ \\
\hline Post-index & $\begin{array}{c}135 \\
(47.7 \%)\end{array}$ & $\begin{array}{c}115 \\
(49.8 \%)\end{array}$ & $\begin{array}{c}57^{\mathrm{T}} \\
(53.7 \%)\end{array}$ & $\begin{array}{c}91^{\mathrm{Q}} \\
(44.3 \%)\end{array}$ \\
\hline
\end{tabular}

\footnotetext{
${ }^{\mathrm{O}}$ Significant difference between quetiapine and olanzapine cohorts at $\mathrm{p}<0.05$

${ }^{\mathrm{R}}$ Significant difference between quetiapine and risperidone cohorts at $\mathrm{p}<0.05$

${ }^{\mathrm{T}}$ Significant difference between quetiapine and typicals cohorts at $\mathrm{p}<0.05$
} 
Results for research objective 14

For schizophrenia and bipolar disorder patients, to compare the impact of quetiapine with risperidone, olanzapine, and typical antipsychotics on patient's adherence to the index medications in the post-index period.

\section{$\underline{\text { Schizophrenia }}$}

Results of univariate analysis presented in table 50 show that there was a significant difference in the average medication possession ratio between quetiapine $(0.78 \pm 0.25)$ and typical antipsychotic cohort $(0.55 \pm 0.32)$. Results of the multivariate analysis presented in table showed that patients initiated on typical antipsychotics were about $22 \%$ less adherent than patients initiated on quetiapine (Table 51).

\section{$\underline{\text { Bipolar Disorder }}$}

Results of univariate analysis presented in table 52 show that there was a significant difference in the average medication possession ratio between quetiapine $(0.71 \pm 0.25)$ and typical antipsychotic cohort $(0.46 \pm 0.34)$. Results of the multivariate analysis presented in table showed that patients initiated on typical antipsychotics were about $24.4 \%$ less adherent than patients initiated on quetiapine (Table 53). 
Table 50: Univariate comparison of medication possession ratio (mpr) among schizophrenia patients

$\begin{array}{cccc}\text { Olanzapine } & \text { Risperidone } & \text { Quetiapine } & \text { Typical } \\ N=346 & N=201 & N=149 & N=303\end{array}$

\section{Medication Possession Ratio}

(MPR)

\begin{tabular}{|c|c|c|c|c|}
\hline Mean ( $(+$ std) & $\begin{array}{c}0.77 \\
(+0.26)\end{array}$ & $\begin{array}{c}0.75 \\
(+0.27)\end{array}$ & $\begin{array}{c}0.78^{\mathrm{T}} \\
(+0.25)\end{array}$ & $\begin{array}{c}0.55^{Q} \\
(+0.32)\end{array}$ \\
\hline
\end{tabular}

\footnotetext{
${ }^{0}$ Significant difference between quetiapine and olanzapine cohorts at $\mathrm{p}<0.05$

${ }^{\mathrm{R}}$ Significant difference between quetiapine and risperidone cohorts at $\mathrm{p}<0.05$

${ }^{\mathrm{T}}$ Significant difference between quetiapine and typicals cohorts at $\mathrm{p}<0.05$
} 
Table 51: Ordinary Least Squares (OLS) regression model for adherence to the index antipsychotic therapy among schizophrenia patients

\begin{tabular}{|c|c|c|c|c|}
\hline & Beta & S.E & t-statistic & $\begin{array}{c}\text { Significance } \\
(p)\end{array}$ \\
\hline \multicolumn{5}{|l|}{ Demographic characteristics } \\
\hline Age (in years) & 0.001 & 0.001 & 1.250 & 0.212 \\
\hline Males (ref: females) & 0.000 & 0.000 & -0.300 & 0.762 \\
\hline Whites (ref: non-whites) & 0.001 & 0.030 & 0.030 & 0.978 \\
\hline Metro (ref: non-metro) & -0.043 & 0.023 & -1.870 & 0.062 \\
\hline \multicolumn{5}{|l|}{ Prescribing physician type } \\
\hline Psychiatric prescriber & 0.000 & 0.000 & 0.230 & 0.818 \\
\hline \multicolumn{5}{|c|}{ Schizophrenia subtype (ref: only schizophrenia) } \\
\hline Schizoaffective disorder & -0.042 & 0.024 & -1.730 & 0.084 \\
\hline $\begin{array}{l}\text { Both schizoaffective disorder } \\
\text { and schizophrenia }\end{array}$ & -0.046 & 0.025 & -1.810 & 0.070 \\
\hline \multicolumn{5}{|l|}{ Pre-index co-morbidities } \\
\hline Alcohol and substance abuse & -0.036 & 0.023 & -1.580 & 0.114 \\
\hline Bipolar disorder & -0.008 & 0.030 & -0.270 & 0.784 \\
\hline Major depression & 0.057 & 0.025 & 2.260 & 0.024 \\
\hline Mild to Moderate depression & -0.002 & 0.030 & -0.080 & 0.938 \\
\hline Other mental comorbidities & 0.002 & 0.022 & 0.090 & 0.931 \\
\hline diabetes & -0.021 & 0.033 & -0.620 & 0.537 \\
\hline hyperlipedemia & 0.064 & 0.037 & 1.720 & 0.085 \\
\hline hypertension & -0.070 & 0.027 & -2.620 & 0.009 \\
\hline Charlson Comorbidity Index & -0.009 & 0.008 & -1.160 & 0.246 \\
\hline \multicolumn{5}{|c|}{ Pre-index period psychiatric medication use (days of supply) } \\
\hline Mood stabilizers & 0.000 & 0.000 & 2.060 & 0.039 \\
\hline Antipsychotics & 0.000 & 0.000 & 0.800 & 0.424 \\
\hline Antidepressants & 0.000 & 0.000 & 1.780 & 0.075 \\
\hline Benzodiazepines & 0.000 & 0.000 & 0.270 & 0.790 \\
\hline
\end{tabular}


Table 51: Ordinary Least Squares (OLS) regression model for adherence to the index antipsychotic therapy among schizophrenia patients (contd.)

\begin{tabular}{|c|c|c|c|c|}
\hline & Beta & S.E & t-statistic & $\begin{array}{l}\text { Significance } \\
(p)\end{array}$ \\
\hline \multicolumn{5}{|c|}{ Pre-index healthcare utilization and cost } \\
\hline $\begin{array}{l}\text { Number of psychotherapy } \\
\text { visits }\end{array}$ & 0.001 & 0.002 & 0.270 & 0.788 \\
\hline $\begin{array}{l}\text { Number of medication } \\
\text { management visits }\end{array}$ & -0.011 & 0.003 & -3.560 & 0.000 \\
\hline $\begin{array}{l}\text { Number of pre-index mental- } \\
\text { health related ER visits }\end{array}$ & -0.022 & 0.019 & -1.130 & 0.261 \\
\hline $\begin{array}{l}\text { Pre-index mental-health } \\
\text { related physician visits }\end{array}$ & -0.005 & 0.007 & -0.690 & 0.492 \\
\hline $\begin{array}{l}\text { Number of pre-index mental- } \\
\text { health related hospitalizations }\end{array}$ & 0.027 & 0.010 & 2.870 & 0.004 \\
\hline \multicolumn{5}{|c|}{ Year of index prescription (ref: 1999) } \\
\hline - 2000 & 0.020 & 0.025 & 0.810 & 0.419 \\
\hline - 2001 & 0.043 & 0.029 & 1.490 & 0.138 \\
\hline \multicolumn{5}{|c|}{ Index prescription (ref: quetiapine) } \\
\hline Risperidone & -0.026 & 0.032 & -0.800 & 0.421 \\
\hline Olanzapine & -0.011 & 0.028 & -0.370 & 0.711 \\
\hline Typicals & -0.220 & 0.031 & -7.190 & $<.0001$ \\
\hline
\end{tabular}

\section{Model fit statistics:}

Adjusted R-square $=12.2 \%, \mathbf{F}=5.57, \mathbf{p}<0.0001$ 
Table 52: Univariate comparison of medication possession ratio (mpr) among bipolar disorder patients

\begin{tabular}{|c|c|c|c|c|}
\hline & $\begin{array}{c}\text { Olanzapine } \\
\quad N=313\end{array}$ & $\begin{array}{l}\text { Risperidone } \\
\quad N=231\end{array}$ & $\begin{array}{c}\text { Quetiapine } \\
N=106\end{array}$ & $\begin{array}{l}\text { Typical } \\
N=205\end{array}$ \\
\hline \multicolumn{5}{|c|}{$\begin{array}{l}\text { Medication Possession Ratio } \\
\text { (MPR) }\end{array}$} \\
\hline $\begin{array}{l}\text { Mean } \\
( \pm \text { std })\end{array}$ & $\begin{array}{c}0.68 \\
( \pm 0.27)\end{array}$ & $\begin{array}{c}0.68 \\
( \pm 0.27)\end{array}$ & $\begin{array}{l}0.71 \text { Q } \\
( \pm 0.25)\end{array}$ & $\begin{array}{l}0.46^{\mathrm{T}} \\
( \pm 0.34)\end{array}$ \\
\hline
\end{tabular}

${ }^{\mathrm{O}}$ Significant difference between quetiapine and olanzapine cohorts at $\mathrm{p}<0.05$

${ }^{\mathrm{R}}$ Significant difference between quetiapine and risperidone cohorts at $\mathrm{p}<0.05$

${ }^{\mathrm{T}}$ Significant difference between quetiapine and typicals cohorts at $\mathrm{p}<0.05$ 
Table 53: Ordinary Least Squares (OLS) regression model for adherence to the index antipsychotic therapy among bipolar disorder patients

\begin{tabular}{|c|c|c|c|c|}
\hline & Beta & S.E & t-statistic & $\begin{array}{l}\text { Significance } \\
\text { (p) }\end{array}$ \\
\hline \multicolumn{5}{|l|}{ Demographic characteristics } \\
\hline Age (in years) & 0.001 & 0.001 & 1.420 & 0.157 \\
\hline $\begin{array}{l}18 \text { years or above (ref: less } \\
\text { than } 18 \text { years) }\end{array}$ & 0.038 & 0.026 & 1.460 & 0.145 \\
\hline Males (ref: females) & 0.000 & 0.000 & -1.090 & 0.277 \\
\hline Whites (ref: non-whites) & 0.012 & 0.035 & 0.340 & 0.731 \\
\hline Metro (ref: non-metro) & -0.055 & 0.027 & -2.060 & 0.040 \\
\hline \multicolumn{5}{|l|}{ Prescribing physician type } \\
\hline Psychiatric prescriber & 0.000 & 0.000 & -0.340 & 0.735 \\
\hline \multicolumn{5}{|c|}{ Bipolar Disorder subtype (ref: Bipolar disorder I) } \\
\hline Bipolar disorder II & -0.043 & 0.028 & -1.520 & 0.130 \\
\hline Mixed & -0.040 & 0.028 & -1.440 & 0.150 \\
\hline \multicolumn{5}{|l|}{ Pre-index co-morbidities } \\
\hline Alcohol and substance abuse & -0.055 & 0.026 & -2.160 & 0.031 \\
\hline Bipolar disorder & 0.001 & 0.033 & 0.040 & 0.967 \\
\hline Major depression & 0.042 & 0.027 & 1.510 & 0.131 \\
\hline Mild to Moderate depression & 0.009 & 0.033 & 0.290 & 0.774 \\
\hline Other mental comorbidities & 0.011 & 0.024 & 0.470 & 0.635 \\
\hline diabetes & -0.026 & 0.037 & -0.700 & 0.485 \\
\hline hyperlipedemia & 0.085 & 0.041 & 2.060 & 0.040 \\
\hline hypertension & -0.073 & 0.029 & -2.510 & 0.012 \\
\hline Charlson Comorbidity Index & -0.013 & 0.008 & -1.580 & 0.114 \\
\hline \multicolumn{5}{|c|}{ Pre-index period psychiatric medication use (days of supply) } \\
\hline Mood stabilizers & 0.000 & 0.000 & 2.300 & 0.022 \\
\hline Antipsychotics & 0.000 & 0.000 & 0.620 & 0.534 \\
\hline Antidepressants & 0.000 & 0.000 & 2.310 & 0.021 \\
\hline
\end{tabular}


Table 53: Ordinary Least Squares (OLS) regression model for adherence to the index antipsychotic therapy among bipolar disorder patients (contd.)

\begin{tabular}{|c|c|c|c|c|}
\hline & Beta & S.E & t-statistic & $\begin{array}{l}\text { Significance } \\
\text { (p) }\end{array}$ \\
\hline Benzodiazepines & 0.000 & 0.000 & -0.100 & 0.924 \\
\hline \multicolumn{5}{|c|}{ Pre-index healthcare utilization and cost } \\
\hline $\begin{array}{l}\text { Number of psychotherapy } \\
\text { visits }\end{array}$ & 0.001 & 0.003 & 0.240 & 0.814 \\
\hline $\begin{array}{l}\text { Number of medication } \\
\text { management visits }\end{array}$ & -0.013 & 0.003 & -3.800 & 0.000 \\
\hline $\begin{array}{l}\text { Number of pre-index mental- } \\
\text { health related ER visits }\end{array}$ & -0.019 & 0.020 & -0.940 & 0.347 \\
\hline $\begin{array}{l}\text { Pre-index mental-health } \\
\text { related physician visits }\end{array}$ & -0.007 & 0.007 & -0.890 & 0.372 \\
\hline $\begin{array}{l}\text { Number of pre-index mental- } \\
\text { health related hospitalizations }\end{array}$ & 0.023 & 0.010 & 2.250 & 0.025 \\
\hline \multicolumn{5}{|c|}{ Year of index prescription (ref: 1999) } \\
\hline - 2000 & 0.021 & 0.027 & 0.790 & 0.430 \\
\hline - 2001 & 0.052 & 0.031 & 1.650 & 0.099 \\
\hline \multicolumn{5}{|c|}{ Index prescription (ref: quetiapine) } \\
\hline Risperidone & -0.038 & 0.035 & -1.060 & 0.290 \\
\hline Olanzapine & -0.030 & 0.033 & -0.920 & 0.359 \\
\hline Typicals & -0.244 & 0.036 & -6.760 & $<.0001$ \\
\hline
\end{tabular}

\section{Model fit statistics:}

Adjusted R-square $=15.13 \%, \mathbf{F}=5.47, \mathbf{p}<0.0001$ 


\section{Results for research objective 15}

For schizophrenia and bipolar disorder patients, to compare the impact of quetiapine with risperidone, olanzapine, and typical antipsychotics on subsequent index antipsychotic therapy modification

$\underline{\text { Schizophrenia }}$

Table 54 presents information on modification of index antipsychotic therapy. The average time to modification of therapy for the quetiapine cohort was 228.5 days as compared to 208.2 days for the olanzapine cohort, 201.8 days for the risperidone cohort and 192.8 days for the typical antipsychotic cohort. About $36.91 \%$ of patients in the quetiapine cohort, $50.58 \%$ in the olanzapine cohort, $47.76 \%$ in the risperidone cohort and $74.26 \%$ in the typical antipsychotic cohort modified the index antipsychotic therapy within the 12-month follow-up period.

Figure 6 presents the Kaplan Meier survival curves for time to modification of therapy for all four study cohorts. The survival curves were significantly different as indicated by log rank statistics of $43.86(\mathrm{p}<0.00)$. Cox proportional hazard model presented in table 55 revealed that patients initiated on risperidone were 1.5 times more likely and patients initiated on typical antipsychotics were about 2.5 times more likely to modify therapy i.e discontinue or switch or augment with another antipsychotic (polytherapy) as compared to patients initiated on quetiapine $(\mathrm{p}<.0001)$.

\section{$\underline{\text { Bipolar Disorder }}$}

Table 56 presents information on modification of index antipsychotic therapy. The average time to modification of therapy for the quetiapine cohort was 219.7 days compared to 200.9 days for the olanzapine cohort, 194.8 days for the risperidone cohort 
and 179.2 days for the typical antipsychotic cohort. About $38.7 \%$ of patients in the quetiapine cohort, $55.5 \%$ in the olanzapine cohort, $39.8 \%$ in the risperidone cohort and $64.4 \%$ in the typical antipsychotic cohort modified the index antipsychotic therapy within the 12-month follow-up period.

The Kaplan Meier survival curves presented in figure 7 revealed that hazards were different for the typical antipsychotic cohort within 250 days of initiating patients on index antipsychotics and after 250 days of initiation on index antipsychotics. Therefore, an extended Cox proportional hazards model was used to estimate impact of index antipsychotic use on time to modification of therapy. There was no significant difference in the hazard of discontinuation or switch or polytherapy between quetiapine and other antipsychotics in the initial 250 days of therapy. However, patients initiated on typical antipsychotics were about 6.3 times more likely to modify therapy i.e discontinue or switch or augment with another antipsychotic (polytherapy) compared to patients initiated on quetiapine in later 250 days of antipsychotic therapy $(\mathrm{p}<.0001)$. The extended Cox proportional hazard model has been presented in table 57. 
Table 54: Therapy modification among schizophrenia patients

\begin{tabular}{|c|c|c|c|}
\hline $\begin{array}{l}\text { Olanzapine } \\
N=346\end{array}$ & $\begin{array}{l}\text { Risperidone } \\
\quad N=201\end{array}$ & $\begin{array}{l}\text { Quetiapine } \\
N=149\end{array}$ & $\begin{array}{l}\text { Typical } \\
N=303\end{array}$ \\
\hline \multicolumn{4}{|c|}{$\begin{array}{l}\text { Time to index therapy modification in days } \\
\text { Mean }(+ \text { std })\end{array}$} \\
\hline $\begin{array}{c}208.15 \\
( \pm 130.52)\end{array}$ & $\begin{array}{c}201.83 \\
( \pm 128.15)\end{array}$ & $\begin{array}{c}228.53 \\
( \pm 128.59)\end{array}$ & $\begin{array}{c}192.81 \\
( \pm 124.54)\end{array}$ \\
\hline \multicolumn{4}{|c|}{$\begin{array}{l}\text { Patients having therapy modification } \\
\text { N }(\%)\end{array}$} \\
\hline $\begin{array}{c}175 \\
(50.58 \%)\end{array}$ & $\begin{array}{c}96 \\
(47.76 \%)\end{array}$ & $\begin{array}{c}55 \\
(36.91 \%)\end{array}$ & $\begin{array}{c}225 \\
(74.26 \%)\end{array}$ \\
\hline
\end{tabular}


Figure 6: Kaplan-Meier Survival Curves for the time to modification of index antipsychotic prescription in schizophrenia patients

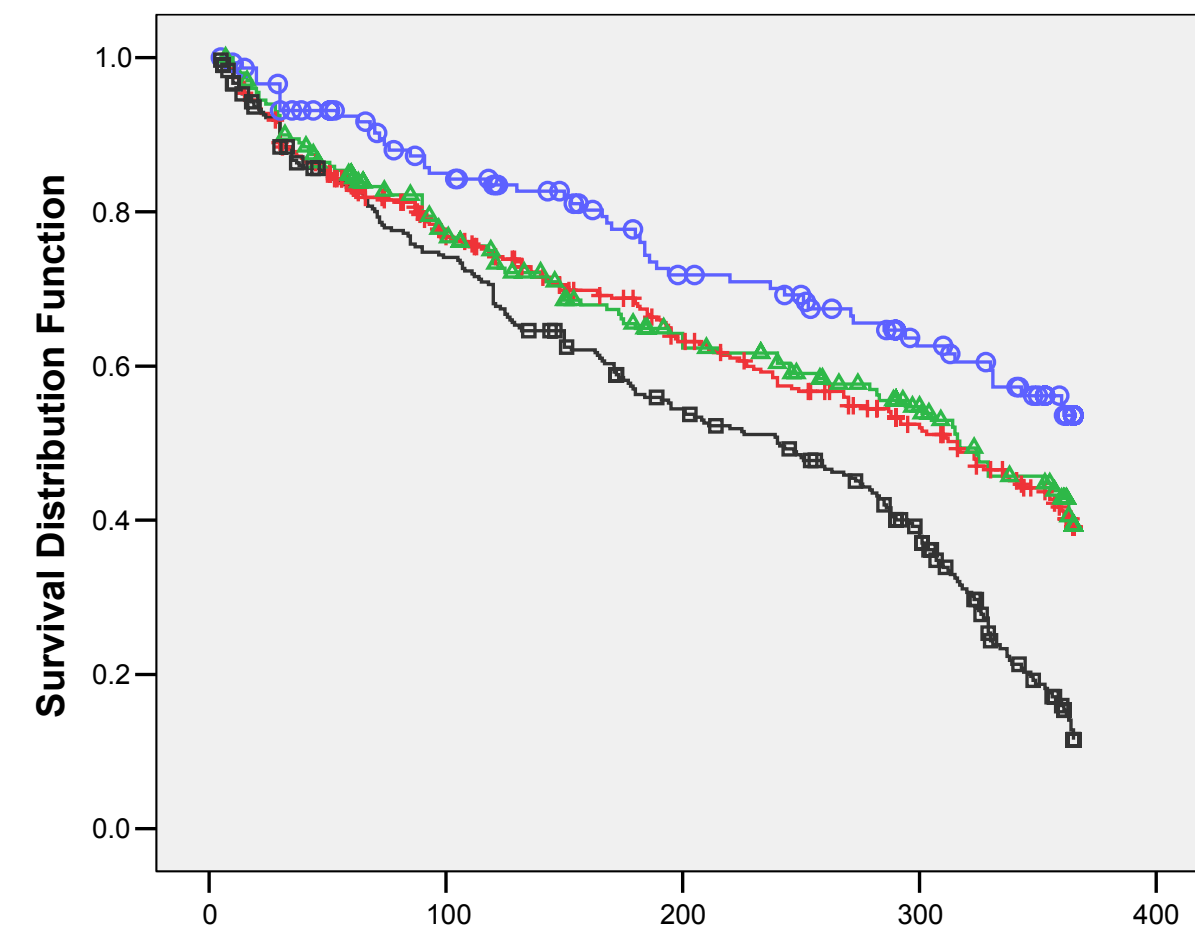

Index Drug
$\Pi$ olan
$\Pi$ quet
$\Pi$ risp
$\Pi$ typ
+ olan-censored
$\Delta$ quet-censored
$\triangle$ risp-censored
$\square$ typ-censored

Time to discontinuation/ switching/ polytherapy in the 12-month follow-up period (in days)

Overall Comparisons

\begin{tabular}{|l|r|r|r|}
\hline & Chi-Square & df & \multicolumn{1}{c|}{ Sig. } \\
\hline Log Rank (Mantel-Cox) & 43.867 & 3 & .000 \\
\hline
\end{tabular}

Test of equality of survival distributions for the different levels of index class. 
Table 55: Cox Proportional hazard model for the impact of index antipsychotic therapy on time to discontinuation/ switch or polytherapy among schizophrenia patients

\begin{tabular}{|c|c|c|c|c|}
\hline \multirow[t]{2}{*}{ Beta } & \multirow[t]{2}{*}{ S.E } & \multirow[t]{2}{*}{$\begin{array}{c}\text { Significance } \\
(p)\end{array}$} & \multirow[t]{2}{*}{$\begin{array}{c}\text { Hazard } \\
\text { Ratio }\end{array}$} & $\begin{array}{r}95 \% C I \\
I\end{array}$ \\
\hline & & & & $\begin{array}{c}\text { Lower } \\
\text { limit }\end{array}$ \\
\hline
\end{tabular}

\begin{tabular}{|c|c|c|c|c|c|c|}
\hline \multicolumn{7}{|c|}{ Demographic characteristics } \\
\hline Age (in years) & -0.010 & 0.005 & 0.065 & 0.990 & 0.980 & 1.001 \\
\hline $\begin{array}{l}\text { Males (ref: } \\
\text { females) }\end{array}$ & -0.101 & 0.117 & 0.389 & 0.904 & 0.719 & 1.137 \\
\hline $\begin{array}{l}\text { Whites (ref: non- } \\
\text { whites) }\end{array}$ & -0.062 & 0.196 & 0.751 & 0.940 & 0.639 & 1.381 \\
\hline $\begin{array}{l}\text { Metro (ref: non- } \\
\text { metro) }\end{array}$ & 0.043 & 0.136 & 0.749 & 1.044 & 0.800 & 1.363 \\
\hline \multicolumn{7}{|c|}{ Prescribing physician type } \\
\hline $\begin{array}{l}\text { Psychiatric } \\
\text { prescriber }\end{array}$ & 0.117 & 0.140 & 0.403 & 1.124 & 0.855 & 1.478 \\
\hline $\begin{array}{l}\text { Schizoaffective } \\
\text { disorder }\end{array}$ & 0.091 & 0.144 & 0.524 & 1.096 & 0.827 & 1.452 \\
\hline $\begin{array}{l}\text { Both } \\
\text { schizoaffective } \\
\text { disorder and } \\
\text { schizophrenia }\end{array}$ & -0.107 & 0.155 & 0.490 & 0.899 & 0.663 & 1.217 \\
\hline \multicolumn{7}{|c|}{ Pre-index co-morbidities } \\
\hline $\begin{array}{l}\text { Alcohol and } \\
\text { substance abuse }\end{array}$ & 0.201 & 0.133 & 0.131 & 1.223 & 0.942 & 1.587 \\
\hline Bipolar disorder & 0.082 & 0.180 & 0.648 & 1.086 & 0.763 & 1.544 \\
\hline Major depression & 0.062 & 0.151 & 0.678 & 1.064 & 0.793 & 1.430 \\
\hline $\begin{array}{l}\text { Mild to Moderate } \\
\text { depression }\end{array}$ & 0.030 & 0.164 & 0.853 & 1.031 & 0.748 & 1.421 \\
\hline $\begin{array}{l}\text { Other mental } \\
\text { comorbidities }\end{array}$ & 0.045 & 0.135 & 0.740 & 1.046 & 0.803 & 1.363 \\
\hline Diabetes & -0.141 & 0.194 & 0.468 & 0.869 & 0.595 & 1.270 \\
\hline Hyperlipedemia & 0.077 & 0.220 & 0.728 & 1.080 & 0.701 & 1.663 \\
\hline Hypertension & 0.193 & 0.156 & 0.214 & 1.213 & 0.894 & 1.646 \\
\hline
\end{tabular}


Table 55: Cox Proportional hazard model for the impact of index antipsychotic therapy on time to discontinuation/ switch or polytherapy among schizophrenia patients (contd.)

\begin{tabular}{|c|c|c|c|c|}
\hline \multirow[t]{2}{*}{ Beta } & \multirow[t]{2}{*}{ S.E } & \multirow[t]{2}{*}{$\begin{array}{c}\text { Significance } \\
\text { (p) }\end{array}$} & \multirow[t]{2}{*}{$\begin{array}{c}\text { Hazard } \\
\text { Ratio }\end{array}$} & $\begin{array}{c}\text { 95\% CI for Hazard } \\
\text { Ratio }\end{array}$ \\
\hline & & & & $\begin{array}{c}\text { Lower } \\
\text { limit }\end{array}$ \\
\hline
\end{tabular}

Charlson

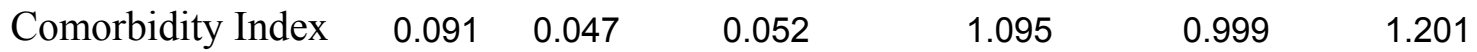

Pre-index period psychiatric medication use (days of supply)

$\begin{array}{lllllll}\text { Mood stabilizers } & 0.000 & 0.000 & 0.407 & 1.000 & 0.999 & 1.001 \\ \text { Antipsychotics } & -0.002 & 0.002 & 0.353 & 0.998 & 0.995 & 1.002 \\ \text { Antidepressants } & 0.000 & 0.000 & 0.166 & 1.000 & 1.000 & 1.001 \\ \text { Benzodiazepines } & 0.000 & 0.001 & 0.546 & 1.000 & 0.999 & 1.001\end{array}$

Pre-index healthcare utilization and cost

Number of

psychotherapy

visits

$0.000 \quad 0.013 \quad 0.990$

1.000

0.976

1.025

Number of

medication

$\begin{array}{lllllll}\text { management visits } & -0.030 & 0.020 & 0.124 & 0.970 & 0.933 & 1.008\end{array}$

Number of pre-

index mental-

health related ER

visits

$\begin{array}{lll}-0.020 & 0.097 & 0.835\end{array}$

0.980

0.811

1.184

Pre-index mental-

health related

physician visits

$0.012 \quad 0.036 \quad 0.734$

1.012

0.944

1.086

Number of pre-

index mental-

health related

hospitalizations

$$
\begin{array}{lll}
-0.019 & 0.056 \quad 0.727
\end{array}
$$

0.981

0.879

1.094

Year of index prescription (ref: 1999)

$\begin{array}{lllllll}\text { - } 2000 & 0.269 & 0.140 & 0.055 & 1.309 & 0.994 & 1.723 \\ \text { - } 2001 & 0.189 & 0.170 & 0.265 & 1.208 & 0.867 & 1.684\end{array}$

Index prescription (ref: quetiapine)

Risperidone

$0.411 \quad 0.204$

0.045

$1.508 \quad 1.010$

2.251 
Table 55: Cox Proportional hazard model for the impact of index antipsychotic therapy on time to discontinuation/ switch or polytherapy among schizophrenia patients (contd.)

\begin{tabular}{lcccccc}
\hline Beta & S.E & $\begin{array}{c}\text { Significance } \\
(\boldsymbol{p})\end{array}$ & $\begin{array}{c}\text { Hazard } \\
\text { Ratio }\end{array}$ & \multicolumn{2}{c}{$\begin{array}{c}\text { 95\% CIfor Hazard } \\
\text { Ratio }\end{array}$} \\
\cline { 5 - 7 } & & & & & $\begin{array}{c}\text { Lower } \\
\text { limit }\end{array}$ & $\begin{array}{c}\text { Upper } \\
\text { Limit }\end{array}$ \\
\hline Olanzapine & 0.248 & 0.182 & 0.173 & 1.281 & 0.897 & 1.830 \\
Typicals & 0.913 & 0.189 & $<.0001$ & 2.491 & 1.721 & 3.605 \\
\hline
\end{tabular}

Model fit statistics:

-2 Log Likelihood $=3911.63$, chi-square $=56.10, p<0.002$ 
Table 56: Therapy modification among bipolar disorder patients

\begin{tabular}{|c|c|c|c|}
\hline $\begin{array}{c}\text { Olanzapine } \\
N=283\end{array}$ & $\begin{array}{l}\text { Risperidone } \\
\qquad N=231\end{array}$ & $\begin{array}{l}\text { Quetiapine } \\
N=106\end{array}$ & $\begin{array}{r}\text { Typical } \\
N=205\end{array}$ \\
\hline \multicolumn{4}{|c|}{$\begin{array}{l}\text { Time to index therapy modification in days } \\
\text { Mean }( \pm \text { std })\end{array}$} \\
\hline $\begin{array}{c}200.9 \\
( \pm 130.4)\end{array}$ & $\begin{aligned} & 194.8 \\
&(+127.8)\end{aligned}$ & $\begin{array}{c}219.8 \\
(+128.9)\end{array}$ & $\begin{array}{c}179.2 \\
( \pm 123.0)\end{array}$ \\
\hline \multicolumn{4}{|c|}{$\begin{array}{l}\text { Patients having therapy modification } \\
\text { N (\%) }\end{array}$} \\
\hline $\begin{array}{c}157 \\
(55.47 \%)\end{array}$ & $\begin{array}{c}92 \\
(39.82 \%)\end{array}$ & $\begin{array}{c}41 \\
(38.67 \%)\end{array}$ & $\begin{array}{c}132 \\
(64.39 \%)\end{array}$ \\
\hline
\end{tabular}


Figure 7: Kaplan-Meier Survival Curves for time to modification of index antipsychotic prescription in bipolar disorder patients

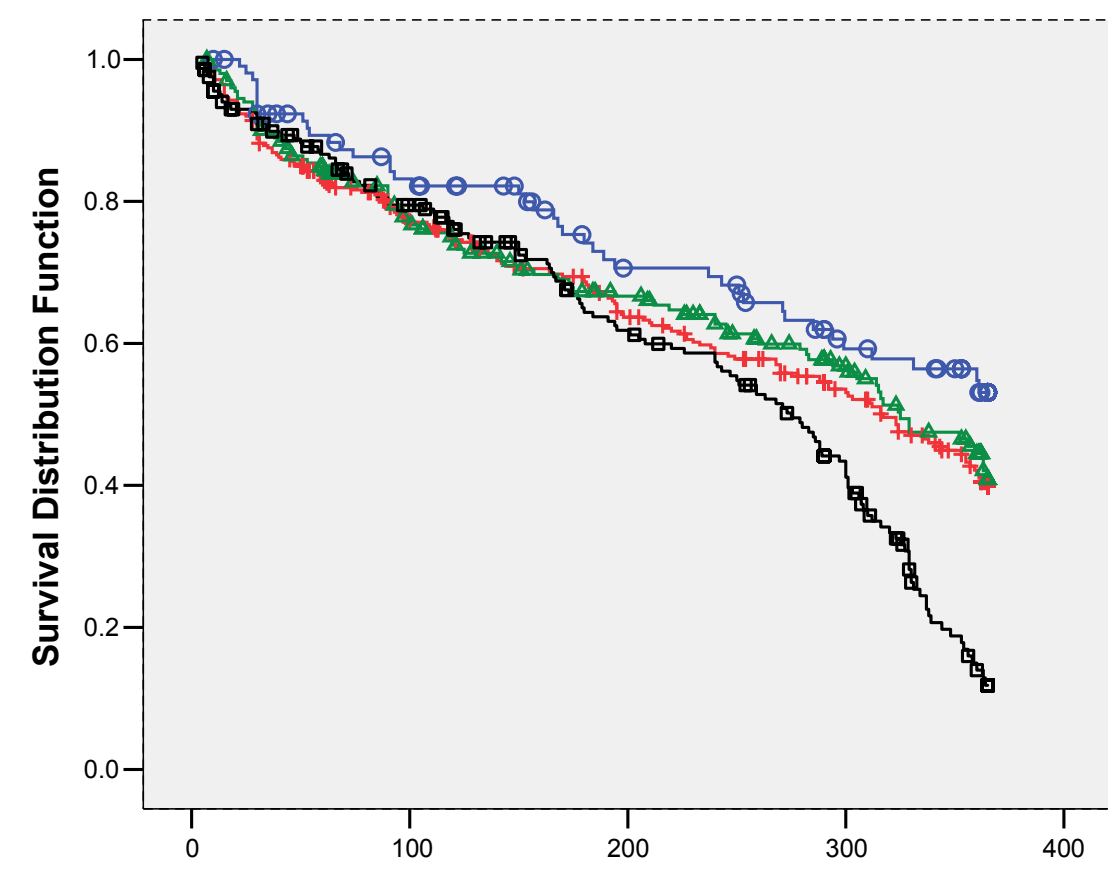
Index Drug
$\neg$ olan
$\neg$ quet
$\sqcap$ risp
$\sqcap$ typ
+ olan-censored
quet-censored
$\triangle$ risp-censored
$\square$ typ-censored

Time to discontinuation/ switching / polytherapy in the 12-month follow up period (in days)

Overall Comparisons

\begin{tabular}{|l|r|r|r|}
\hline & Chi-Square & df & \multicolumn{1}{c|}{ Sig. } \\
\hline Log Rank (Mantel-Cox) & 27.155 & 3 & .000 \\
\hline
\end{tabular}

Test of equality of survival distributions for the different levels of index class. 
Table 57: Extended Cox Proportional hazard model for the impact of index antipsychotic therapy on time to discontinuation/ switch or polytherapy among bipolar disorder patients

\begin{tabular}{cccccc} 
Beta & S.E & Significance & Hazard & & \multicolumn{2}{c}{$95 \%$} & CIfor Hazard \\
& $(p)$ & Ratio & & \multicolumn{2}{c}{ Ratio } \\
\cline { 3 - 5 } & & & Lower & Upper \\
& & & & limit & Limit
\end{tabular}

\section{Demographic characteristics}

$\begin{array}{lllllll}\text { Age (in years) } & -0.009 & 0.006 & 0.122 & 0.991 & 0.979 & 1.000\end{array}$

18 years or above

(ref: less than 18

years)

$\begin{array}{lll}-0.258 & 0.161 & 0.109\end{array}$

0.773

0.563

1.059

Males (ref:

females)

0.131

0.187

0.842

0.651

1.087

Whites (ref: non-

whites)

$0.215 \quad 0.236 \quad 0.362$

1.240

0.780

1.970

Metro (ref: non-

metro)

$\begin{array}{lll}0.005 & 0.159 & 0.973\end{array}$

1.005

0.737

1.372

\section{Prescribing physician type}

Psychiatric

prescriber

0.131

0.161

0.415

1.140

0.832

1.562

Bipolar Disorder subtype (ref: Bipolar disorder I)

Bipolar disorder I
0.090
0.168
0.590

1.094

0.788

1.520

Mixed

$-0.126$

0.175

0.471

0.882

0.626

1.242

\section{Pre-index co-morbidities}

\begin{tabular}{lllllll} 
Alcohol and & & & & & & \\
substance abuse & 0.338 & 0.155 & 0.029 & 1.402 & 1.035 & 1.901 \\
$\begin{array}{l}\text { Bipolar disorder } \\
\text { Major depression }\end{array}$ & 0.126 & 0.206 & 0.540 & 1.134 & 0.758 & 1.699 \\
$\begin{array}{l}\text { Mild to Moderate } \\
\text { depression }\end{array}$ & 0.045 & 0.168 & 0.124 & 1.295 & 0.932 & 1.800 \\
$\begin{array}{l}\text { Other mental } \\
\text { comorbidities }\end{array}$ & -0.045 & 0.156 & 0.774 & 0.956 & 0.705 & 1.298 \\
$\begin{array}{l}\text { Diabetes } \\
\text { Hyperlipedemia }\end{array}$ & 0.062 & 0.216 & 0.775 & 1.064 & 0.697 & 1.623 \\
Hypertension & 0.078 & 0.251 & 0.755 & 0.925 & 0.565 & 1.512 \\
\hline
\end{tabular}


Table 57: Extended Cox Proportional hazard model for the impact of index antipsychotic therapy on time to discontinuation/ switch or polytherapy among bipolar disorder patients (contd.)

Beta S.E

S.E Significance Hazard

(p)

Ratio

95\% CI for Hazard

\begin{tabular}{cc}
\hline Lower & Upper \\
limit & Limit
\end{tabular}

Charlson

Comorbidity Index $\quad-0.041 \quad 0.055 \quad 0.462$

0.960

0.861

1.070

Pre-index period psychiatric medication use (days of supply)

$\begin{array}{lllllll}\text { Mood stabilizers } & -0.001 & 0.001 & 0.068 & 0.999 & 0.998 & 1.000 \\ \text { Antipsychotics } & 0.001 & 0.002 & 0.479 & 1.001 & 0.998 & 1.005 \\ \text { Antidepressants } & 0.000 & 0.000 & 0.389 & 1.000 & 1.000 & 1.001 \\ \text { Benzodiazepines } & -0.001 & 0.001 & 0.388 & 0.999 & 0.998 & 1.001\end{array}$

Pre-index healthcare utilization and cost

Number of

psychotherapy

visits

$\begin{array}{lll}-0.008 & 0.014 & 0.585\end{array}$

0.992

0.964

1.021

Number of

medication

management visits

$\begin{array}{lll}-0.018 & 0.022 \quad 0.408\end{array}$

0.982

0.942

1.025

Number of pre-

index mental-

health related ER

visits

$\begin{array}{lll}-0.093 & 0.103 & 0.369\end{array}$

0.911

0.745

1.116

Pre-index mental-

health related

physician visits

$\begin{array}{lll}0.013 & 0.041 & 0.751\end{array}$

1.013

0.935

1.098

Number of pre-

index mental-

health related

hospitalizations

$$
\begin{array}{ll}
-0.010 & 0.060
\end{array}
$$

0.873

0.990

0.881

1.114

Year of index prescription (ref: 1999)

- 2000

$0.204 \quad 0.157$

0.193

1.226

0.902

1.666

- 2001

$0.268 \quad 0.189$

0.156

1.307

0.903

1.893

Index prescription (ref: quetiapine)

Risperidone

$0.347 \quad 0.231$

0.133

1.415

0.900

2.223 
Table 57: Extended Cox Proportional hazard model for the impact of index antipsychotic therapy on time to discontinuation/ switch or polytherapy among bipolar disorder patients (contd.)

\begin{tabular}{|c|c|c|c|c|c|}
\hline \multirow[t]{2}{*}{ Beta } & \multirow[t]{2}{*}{ S.E } & \multirow[t]{2}{*}{$\begin{array}{c}\text { Significance } \\
(p)\end{array}$} & \multirow[t]{2}{*}{$\begin{array}{c}\text { Hazard } \\
\text { Ratio }\end{array}$} & \multicolumn{2}{|c|}{$\begin{array}{c}\text { 95\% CI for Hazard } \\
\text { Ratio }\end{array}$} \\
\hline & & & & $\begin{array}{l}\text { Lower } \\
\text { limit }\end{array}$ & $\begin{array}{l}\text { Upper } \\
\text { Limit }\end{array}$ \\
\hline
\end{tabular}

\begin{tabular}{|c|c|c|c|c|c|c|}
\hline Olanzapine & 0.232 & 0.210 & 0.270 & 1.261 & 0.835 & 1.903 \\
\hline \multicolumn{7}{|l|}{ Typicals } \\
\hline \multicolumn{7}{|l|}{ (less than 250} \\
\hline days) & 0.238 & 0.274 & 0.386 & 1.268 & 0.741 & 2.171 \\
\hline \multicolumn{7}{|l|}{ Typicals } \\
\hline \multicolumn{7}{|l|}{ (equal or more } \\
\hline than 250 days) & 1.847 & 0.255 & $<.0001$ & 6.338 & 3.844 & 10.452 \\
\hline
\end{tabular}

Model fit statistics:

-2 Log Likehood $=3072.98, \chi^{2}=101.17, p<0.0001$ 


\section{PHASE 2 - Discussion}

The aim of phase II is to compare quetiapine with risperidone, olanzapine, and typical antipsychotics on multiple healthcare outcomes such as healthcare costs, inpatient, outpatient, ER and pharmacy utilization, adherence and treatment modification. Intentto-treat methodology was used for the economic comparisons. Intent to treat methodology includes the costs of patient dropouts due to adverse effects or treatment failure into economic evaluations. Without the intent-to-treat perspective, it will be difficult to include the cost impact of the additional expenses incurred by the patients who do not respond to treatment or fail treatment while comparing different treatment options. Policy makers find studies with intent-to-treat methodology and studies reporting costs of all patients instead only those patients who repond to initial treatment more useful while making healthcare decisions (Croghan, Johnstone, Buesching, \& Kessler, 1999).

\section{Total and mental healthcare costs for schizophrenia and bipolar disorder patients}

The study demonstrated that there were no difference in the total and mental health-related costs between schizophrenia patients initiated on quetiapine and other antipsychotics. These findings were reported after controlling for various confounders such as patient demographics, co-morbidities, pre-index medication use, healthcare utilization and costs. To our knowledge, this is the first study reporting total and mental healthcare cost comparisons of quetiapine with other antipsychotics for schizophrenia patients.

Till date, pharmacoeconomic evaluations of antipsychotics have compared the impact of risperidone, olanzapine and typical antipsychotics on healthcare costs and 
utilizations. Most of these studies have also not found any significant difference in total and mental healthcare costs between the different antipsychotic treatments. The lack of difference is mainly due to variable effect of the antipsychotic use on healthcare component costs such as inpatient costs, outpatient costs, and pharmacy costs. Usually the high pharmacy costs associated with atypical antipsychotics were offset by lesser inpatient and outpatient costs. For example, Edgell and colleagues reported that olanzapine had higher pharmacy costs and lower inpatient costs compared to risperidone resulting in no significant difference in total healthcare costs (Edgell et al., 2000). Gibson and colleagues also report no significant difference in total healthcare costs between olanzapine, risperidone and typical antipsychotics (Gibson et al., 2004). However, after excluding the cost of index prescription, they found that the total costs associated with olanzapine were significantly lower than risperidone. Few other studies have reported varying results. A study by Zhao and colleagues comparing risperidone and olanzapine use among uncontrolled schizophrenia population from a employer-based insurance population reported lower total costs for olanzapine than risperidone (Zhao, 2004). On the other hand, a study by Rascati and colleagues comparing olanzapine and risperidone in a Texas Medicaid population reported lower costs for risperidone as compared to olanzapine (Rascati et al., 2003).

The PRIZE (the Partial Responders International schizophrenia Evaluation) study conducted in a UK population as compared quetiapine treatment to haloperidol in terms of clinical endpoints (Emsley, Raniwalla, Bailey, \& Jones, 2000). A markov model based on this study results estimated similar total costs with quetiapine $(£ 38,106)$ compared to haloperidol (£38,350) (Tilden et al., 2002). This study reported that higher 
acquisition costs of quetiapine were offset by higher inpatient and outpatient costs of haloperidol. In present study, the impact of the index antipsychotic on each component of healthcare costs were evaluated to examine cost offsets among these healthcare components.

Total healthcare costs evaluation for bipolar disorder patients found no significant differences between quetiapine and other index antipsychotics. However, evaluation of mental healthcare costs for bipolar disorder patients revealed that patients initiated on quetiapine incur lower mental healthcare costs compared to patients initiated on typical antipsychotics. There were no significant differences in mental healthcare costs between quetiapine and other atypical antipsychotics.

There is only one other study that compared the impact of antipsychotics on healthcare costs and utilization in bipolar disorder patients. This study reported that patients on quetiapine incurred $\$ 76$ lesser mental-health related charges per patient per month compared to olanzapine. There was no significant difference in costs between risperidone and quetiapine (Gianfrancesco et al., 2005). This study did not include comparison with typical antipsychotics. The difference in results between our study and the study by Gianfrancesco may be due to differences in patient population and study methodology. The study by Gianfrancesso was conducted in a private health plan population using treatment episode methodology. Comparisons between the antipsychotics in the study by Gianfrancesso were based on costs incurred between the initiation of therapy and discontinuation with the therapy. Therefore, it did not consider costs associated with treatment discontinuation or adverse effects in the analysis. With the use of treatment episode methodology, it is possible that a person who has not 
stabilized on earlier antipsychotic therapy may get initiated on another antipsychotic in the next episode and incur higher costs. Therefore, treatment episodes with newer antipsychotics may have higher costs due to inclusion of more severe patients.

\section{Mental health-related inpatient utilization and cost}

Our study found no significant difference in inpatient costs between quetiapine and other atypical antipsychotics. However, mental health-related inpatient costs among patients initiated on typical antipsychotics were higher than those of patients initiated on quetiapine. The Markov model by Tilden and colleagues had also estimated lower inpatient costs with quetiapine (£22,798) as compared to haloperidol (£24,716) (Tilden et al., 2002). Two other studies comparing costs between the atypical antipsychotics (olanzapine and risperidone) have also reported similar inpatient costs between the atypical antipsychotics.(Byerly et al., 2003; Jerrell, 2002).

Analysis of inpatient utilization revealed that there were no significant differences in the proportion of schizophrenia patient who were hospitalized and number of inpatient visits between quetiapine and typical antipsychotic cohorts. A study by Jerrell and colleagues also reported no significant difference in time to hospitalization and number of hospitalizations in patients initiated on risperidone, olanzapine and typical antipsychotics (Jerrell, 2002).

Our study did not find any significant difference in mental health-related inpatient utilization and costs between quetiapine and other antipsychotics in bipolar disorder patients. These results are consistent with the findings of the study by Gianfrancesso reporting no significant differences in inpatient costs (Gianfrancesco et al., 2005). It is likely that among bipolar disorder patients antipsychotics are being used as an adjunct to 
other medications like mood stabilizers and antidepressants to manage patients.

Therefore, there may not be a significant impact of antipsychotic on inpatient utilization by itself.

\section{Mental health-related ER and outpatient utilization and cost}

Our study categorized the outpatient costs into ER and outpatient costs. We found that there was no significant difference between quetiapine and other antipsychotics in terms of ER costs and ER utilization for both schizophrenia and bipolar disorder patients. Study of outpatient utilization is complex for mental health patients as outpatient utilization can be psychotherapy, psychotherapy tests, routine physician visits or emergency room visits. Utilization of outpatient services can also depend upon access to mental health clinics or hospitals which may be lacking in many places especially rural areas. Many patients lacking access to appropriate healthcare service may use ER for routine care services (Lehman \& Steinwachs, 1998).

Studies that categorize healthcare utilization usually separate them into inpatient and outpatient services. ER utilization and costs are usually aggregated with those of other outpatient services (Byerly et al., 2003; Jerrell, 2002). This may be due to lower utilization rates for ER services compared to other services.

In our study, comparison of mental health-related physician visits, psychotherapy and medication management visits revealed there were significant differences between quetiapine and other antipsychotics among both schizophrenia and bipolar disorder patients. Multivariate analysis was conducted for the outpatient costs and physician visits. Though there were no significant differences between the cohorts in terms of number of mental health-related physician visits, the outpatient costs were slightly higher 
for patients initiated on typical antipsychotics as compared to patients initiated on quetiapine. The utilization of psychotherapy sessions and medication management visits are probably reflected in the analysis of outpatient costs.

There were no significant differences in outpatient costs between quetiapine and other atypical antipsychotics in schizophrenia and bipolar disorder patients. However, the outpatient costs of typical antipsychotic were slightly higher than quetiapine. Markov modeling by Tinden and colleagues estimated higher outpatient costs for typicals (£9,698) as compared to quetiapine $(£ 9,036)$ in the schizophrenia population. This may be due to better treatment response and lesser side-effects associated with quetiapine (Tilden et al., 2002). Comparison of outpatient costs among schizophrenia patients by Jerrell and colleagues also found no difference between the atypical antipsychotics risperidone and olanzapine (Jerrell, 2002). However, interpreting the impact of antipsychotic on outpatient costs can be complicated. Schizophrenia and bipolar disorder patients require regular care not only in the acute phases of disease but also in the maintenance phase. Many patients with schizophrenia and bipolar disorder are also treated for substance abuse in outpatient settings. Therefore, utilization of nonemergency services may also be an indicator of appropriate treatment and patient management that may improve patient outcomes in future (American Psychiatric Association, 1997; Lehman et al., 2004a; Lehman et al., 2004c).

\section{Mental health-related pharmacy utilization and cost}

Our study found that the pharmacy costs for patients initiated on olanzapine were significantly higher than quetiapine in both the schizophrenia and bipolar disorder populations. This may be result of higher acquisition costs of olanzapine, greater use of 
concomitant medications in the olanzapine cohort, or better adherence or persistence with olanzapine therapy. Further analysis of number of days of therapy with the index antipsychotic revealed no significant difference between days of therapy with olanzapine and quetiapine in the post-index period. Therefore, a higher pharmacy cost in the olanzapine cohort compared to quetiapine is not due to better adherence or persistence with olanzapine therapy. Uses of concomitant medications were similar for psychiatric drugs between olanzapine and quetiapine cohort. Even after controlling for use of concomitant medications as well as other confounding factors such as patient demographics and co-morbidities, the difference in pharmacy costs between quetiapine and olanzapine was still significant suggesting a very high acquisition cost for olanzapine. As expected, the pharmacy costs of quetiapine were significantly higher than typical antipsychotics mainly due to higher acquisition costs for quetiapine.

Though the drug acquisition cost for each healthcare system may differ, the Average Wholesale Price (AWP) per month is higher for olanzapine (approx: \$599) compared to risperidone (approx: \$385), quetiapine (approx: \$351) and typical antipsychotics (approx: \$40) according to the Red Book. This is reflected in the results of all studies comparing pharmacy costs of antipsychotics. Most studies have compared inpatient pharmacy costs between antipsychotics. Kasper and colleagues reported significantly higher average total inpatient drug costs for olanzapine (\$297.5) as compared to risperidone (\$159.9) based on retrospective chart reviews of hospitalized schizophrenia patients (Kasper et al., 2001). Analysis of daily drug costs from an inpatient data by Kelly and colleagues found patients initiated on risperidone incurred lower drug costs (\$6.42) compared to schizophrenia patients initiated on olanzapine 
(\$12.29) (Kelly et al., 2001). Analysis of VA data revealed that there are no significant differences in inpatient and outpatient costs among schizophrenia patients initiated on risperidone or olanzapine, and therefore it is more economic to prescribe risperidone due to its lower drug acquisition costs (Byerly et al., 2003). Similarly, Gibson and colleagues report that after subtracting the cost of index antipsychotic from total costs, post-index cost of olanzapine treated schizophrenia patient cohort was lower than risperidone treated schizophrenia cohort. None of these analyses have included quetiapine in their cost comparisons.

The only study reporting cost comparisons among schizophrenia patients for quetiapine examined total pharmacy costs in an acute care inpatient medical setting. Based on retrospective chart reviews of patients initiated on olanzapine, risperidone or quetiapine, the study reported that the average daily pharmacy cost was $\$ 4.35$ less for risperidone and $\$ 1.41$ less for quetiapine as compared to olanzapine (Mladsi et al., 2004). This study has certain limitations. The results of this study apply only to those patients who respond to the treatment as the study sample included only those patients who were discharged within 30 days of hospitalizations. In addition, the study did not control for use of concomitant medications during the inpatient stay. However, the results of all the studies suggest that patients initiated on olanzapine do incur higher pharmacy costs.

The only study reporting pharmacy costs for bipolar disorder patients on antipsychotics was the study by Gianfrancesso using the treatment episode mythology. Despite the difference in methodology from our study, this study reports results similar to ours that the per-patient per-month pharmacy costs of olanzapine group was $49 \%$ more than that of quetiapine group (Gianfrancesco et al., 2005). 
Our study found that use of concomitant medications was highly prevalent among schizophrenia and bipolar disorder patients in both pre-index as well as post-index period. Few studies have reporting detailed utilization of concomitant medication use in these patient populations. A study by Al-Zakhwani and colleagues reported greater use of mood stabilizers among patient on atypical antipsychotic (44.5\%) as compared to patient on typical antipsychotics (31.88\%). The use of antidepressants was also more prevalent among atypical users (81.0\%) compared to typical users (51.8\%) (Al Zakwani et al., 2003). Menzin and colleagues reported lower likelihood of receiving anxiolytics $(\mathrm{OR}=0.44)$ and anticholinergics $(\mathrm{OR}=0.15)$ among patients on atypical antipsychotics compared to patients on typical antipsychotics (Menzin et al., 2003). Greater use of anticholinergic drugs for typical antipsychotics is indicative of need for treatment of extra pyramidal symptoms among patients initiated on typical antipsychotics.

Pre-index and post-index prevalence of mood stabilizer use was similar among all study antipsychotic drugs for bipolar disorder patients initiated on antipsychotics. Pre and post index prevalence of benzodiazepine use was very high among bipolar disorder patients initiated on quetiapine. However, there was a very slight change in the proportion of patients on benzodiazepine in the post-index period from the pre-index period. Proportions of patients on anxiolytics/hypnotics/sedatives and antidepressants were very high across all study cohorts. However, proportion of patients on antiparkinson and anticholinergics were not very high across study antipsychotic cohorts among bipolar disorder patients as compared to schizophrenia patients. The study by Gainfrancesso did not find any significant differences in concomitant medication use between risperidone, olanzapine and quetiapine in bipolar disorder patients. 


\section{Index antipsychotic adherence and therapy modification}

Though there was no significant difference in adherence measured in terms of medication possession ratio between quetiapine and atypical antipsychotics, there were significant differences in adherence between quetiapine and typical antipsychotics for both schizophrenia and bipolar disorder. Published studies have shown lower adherence for typical antipsychotics due to side-effects such as extrapyramidal symptoms and tardive dyskinesia (Crismon ML et al., 1997; APA, 1997; Carpenter, Jr. et al., 1994). A study by Al-Zakhwani and colleagues compared persistence with index therapy and medication possession ratio between typicals and atypical antipsychotics in a patient population with psychosis (Al Zakwani et al., 2003). They reported that average days of supply of atypical antipsychotic in a year were 136 days compared to 80 days with typical antipsychotics. The medication possession ratio reported for atypical antipsychotics was 0.53 compared to 0.24 for typical antipsychotics. Though the lower adherence for typical antipsychotics as compared to atypical antipsychotics is consistent with our study results, the ratio of adherence in their study population was considerably lower than that found in our study (about 0.7 for atypicals and 0.5 for typicals). This may be because their study sample was not restricted to schizophrenia or bipolar disorder population. It is possible that patients in their study may be receiving antipsychotics for short term duration for disease conditions that are not indicated for antipsychotic use.

Another study by Menzin and colleagues conducted in the California Medicaid schizophrenia population compared persistence with therapy, switching and discontinuation between atypical and typical antipsychotics (Menzin et al., 2003). They found that patients initiated on atypical antipsychotics (61\%) had greater days of index 
medication supply in 1-year post-index period compared to typicals (58\%). They also reported higher rates of discontinuation among patients initiated on typicals (58\%) as compared to patients initiated on atypicals (33\%). Though their analysis did not include quetiapine, their results with respect to comparison of atypical with typical are consistent with our study. Dolder and colleagues compared adherence between atypical antipsychotics (including risperidone, olanzapine, or quetiapine) to typical antipsychotics in a California VA population with psychosis. They reported poorer adherence rates with typical antipsychotics (50.1\%) compared to atypical antipsychotics (54.9\%) (Dolder et al., 2002).

To our knowledge, there are no studies comparing quetiapine with other atypical antipsychotics and typical antipsychotics in terms of persistence with therapy, adherence, or therapy modifications such as interrupted, switching and polytherapy. In addition to comparisons of quetiapine with other antipsychotics in terms of persistence with therapy and adherence, this study conducted a detailed analysis examining time to modification of index antipsychotic. Modification of index antipsychotic can take place due to discontinuation of index antipsychotic use, switching to another antipsychotic or augmenting with another antipsychotic (later polytherapy). This study found that the patients initiated on typical antipsychotic had higher the risk of therapy modification as compared to patients initiated on quetiapine among schizophrenia patients. Among bipolar disorder patients, the risk of therapy modification with typical antipsychotics increased 6-fold in the latter part of post-index period. Though specific reasons for therapy modifications such as physicians' judgment or patient preference cannot be ascertained from a retrospective database analysis, therapy modification can have 
implications on costs and utilization (Centorrino et al., 2004; McCombs et al., 2000;

Loosbrock et al., 2003). 


\section{CHAPTER FIVE}

\section{CONCLUSIONS}

This chapter presents conclusions drawn from the study, lists limitations and implications of the study and provides recommendations for future research.

\section{Phase 1}

The primary goal of the phase I was to describe and evaluate antipsychotic utilization patterns among schizophrenia and bipolar disorder patients.

\section{Conclusions from research objective 1}

To determine the annual prevalence rate of schizophrenia and bipolar disorder in West Virginia Medicaid from 1998 to 2002

This was an exploratory question examining the overall prevalence rate of schizophrenia and bipolar disorder among WV Medicaid enrollees from 1998 and 2002. The annual prevalence rates of schizophrenia in the WV Medicaid population from 1998 to 2002 ranged from $0.9 \%$ to $1.5 \%$. These rates were similar to the national estimate of $1.1 \%$ prevalence. The annual prevalence rate for bipolar disorder in the WV Medicaid population from 1998 to 2002 ranged from $0.6 \%$ to $1.7 \%$. These rates were lower than the national estimate of 1.3 to $1.6 \%$ for the year 1998 but similar to the national estimates between the years 1999 and 2000. The annual prevalence rate of bipolar disorder among population between 20 and 64 years of age between the year 2000 and 2002 ranged from $3.2 \%$ to $3.5 \%$ which is similar to the national estimate of $3.7 \%$ among adult US population. 


\section{Conclusions from research objective 2}

To determine the medical conditions for which antipsychotics are being prescribed in the West Virginia Medicaid population as well as describe patterns of distribution of certain demographic factors such as age, gender, and ethnicity in patients using antipsychotics.

This was an exploratory analysis to determine the mental health conditions as well as describe patterns of distribution of certain demographic factors such as age, gender, and ethnicity in patients who were initiated on antipsychotics in the West Virginia Medicaid population. We conclude that there were patients who were initiated on antipsychotics for conditions that are not yet FDA indicated for antipsychotic use such as non-schizophrenic psychosis, attention deficit disorder, major depression, mild to moderate depression, and anxiety. As WV Medicaid population consists of large percentage of Caucasian women, the variation in distribution according to gender and race among patients initiated on antipsychotics followed the general demographic characteristics of WV Medicaid beneficiaries. Therefore, it cannot be concluded from our study that the initiation of antipsychotics varies by gender or race.

\section{Conclusions from research objective 3}

For schizophrenia and bipolar disorder patients, determine different types of utilization pattern of antipsychotics.

The null hypotheses for this research question was there are no differences in the utilization patterns of antipsychotics among patients. This hypothesis was investigated among schizophrenia and bipolar disorder patients. For both schizophrenia and bipolar disorder patients, there were significant number of patients using antipsychotic polytherapy, switching from index antipsychotic, having interrupted/ non-adherent 
therapy with index antipsychotic and having continuous/ adherent therapy with index antipsychotic. Lower proportion of less than 18 years old bipolar disorder patients received continuous/ adherent index antipsychotic therapy compared to bipolar disorder patients who are 18 years or older. As these utilization patterns were defined on the basis of refill claims, they only indicate that the patient has refilled the medication. They do not indicate whether the patient has taken the medication. However, these definitions based on refill claims can be accepted to capture the utilization patterns of antipsychotics as 1) several studies have validated the positive correlation between refill claims and patient diaries and drug blood serum levels and 2) As Medicaid offers complete prescription drug coverage, Medicaid patients may not refill prescriptions outside the system. Therefore, we reject our null hypothesis and conclude from this study that significantly different antipsychotic utilization patterns exist among schizophrenia and bipolar disorder patients.

\section{Conclusions from research objective 4}

For schizophrenia and bipolar disorder patients, determine the gaps between the refills of antipsychotics.

The null hypotheses for this research question was there are no gaps in the antipsychotic therapy among schizophrenia and bipolar disorder patients. Large proportion of schizophrenia and bipolar disorder patients had gaps of greater than 15 days between the refills of antipsychotics. Among bipolar disorder patients, there were differences in duration of gaps and proportions of patients with large gaps between patients who are 18 years or older and patients who are less than 18 years. As Medicaid offers comprehensive coverage for prescriptions, patients in Medicaid are not very likely 
to fill their prescriptions outside the system. Therefore, we can reject our hypothesis and conclude that there are gaps in the antipsychotic therapy among schizophrenia and bipolar disorder patients

\section{Conclusions from research objective 5}

For schizophrenia and bipolar disorder patients, determine predictors of different utilization patterns of antipsychotics

The null hypothesis for this research question was there is no association between utilization patterns of antipsychotics and patient demographics, prescribing physician type, mental health diagnosis, other medical diagnosis, type of antipsychotic, year of index antipsychotic, pre-index concomitant medication use, pre-index alcohol and substance abuse and pre-index healthcare utilization among schizophrenia and bipolar disorder patients. Based on our results the null hypothesis was rejected. We concluded that factors such as schizophrenia subtypes, alcohol and substance abuse, major depression, prior use of mood stabilizers and antidepressants in the pre-index period and use of typical antipsychotic as index antipsychotic were significant predictors of different patterns of utilization of antipsychotics among schizophrenia patients. We also concluded that among bipolar disorder patients, factors such as less than 18 years of age, psychiatric prescriber, alcohol and substance abuse, major depression, anxiety, prior use of mood stabilizers, and use of typical antipsychotic as index antipsychotic were significant predictors of different patterns of utilization of antipsychotics.

\section{Conclusions from research objective 6}

For schizophrenia and bipolar disorder patients, determine the relationship between utilization pattern of antipsychotics and total health-related healthcare costs. 
The null hypothesis was there is no association between utilization patterns of antipsychotics and total health healthcare utilization and costs for schizophrenia and bipolar disorder patients. This null hypothesis was rejected based on our results. We concluded that compared to continuous/adherent antipsychotic therapy with index antipsychotic, patterns of antipsychotic utilization such as polytherapy, and interrupted/ non-adherent therapy were significantly associated with higher total healthcare costs for schizophrenia patients. We also concluded that switching from index antipsychotic was not significantly associated with total healthcare costs compared to continuous/adherent antipsychotic therapy with index antipsychotic for schizophrenia patients. Among bipolar disorder patients, it was concluded that patterns of antipsychotic utilization such as polytherapy, switching from index antipsychotic and interrupted/non-adherent therapy were significantly associated with higher total healthcare costs compared to continuous/adherent antipsychotic therapy with index antipsychotic.

\section{Conclusions from research objective 7}

For schizophrenia and bipolar disorder patients, determine the relationship between utilization pattern of antipsychotics and mental health care utilization and costs.

The null hypothesis was there is no association between utilization patterns of antipsychotics and mental health healthcare utilization and costs for schizophrenia and bipolar disorder patients. Based on the results, we concluded that the patterns of antipsychotic utilization such as polytherapy, and interrupted/ non-adherent therapy were significantly associated with higher mental healthcare costs as compared to continuous/adherent antipsychotic therapy with index antipsychotic, for both schizophrenia and bipolar disorder patients. We also concluded that switching from 
index antipsychotic was not significantly associated with mental healthcare costs compared to continuous/adherent antipsychotic therapy with index antipsychotic among both schizophrenia and bipolar disorder patients.

\section{Phase 2}

The aim of phase II is to compare quetiapine with risperidone, olanzapine, and typical antipsychotics on multiple healthcare outcomes such as healthcare costs, inpatient, outpatient, ER and pharmacy utilization, adherence and treatment modification.

\section{Conclusions from research objective 8}

For schizophrenia and bipolar disorder patients, to compare the impact of quetiapine with risperidone, olanzapine, and typical antipsychotics on total and mental healthcare costs

The null hypothesis was that there is no difference in the impact of quetiapine compared with risperidone, olanzapine, and typical antipsychotics on total and mental healthcare costs for schizophrenia and bipolar disorder patients. After controlling for various confounding such as patient demographics, prescribing physician type, mental health diagnosis, other medical diagnosis, type of antipsychotic, year of index antipsychotic, pre-index concomitant medication use, pre-index alcohol and substance abuse and pre-index healthcare utilization, there were no significant differences in total and mental healthcare costs among patients initiated on quetiapine compared to patients initiated on risperidone, olanzapine, or typical antipsychotics for schizophrenia patients (ref: table). Therefore, the null hypothesis was accepted as true for schizophrenia patients. However, there were significant differences in total and mental healthcare costs among patients initiated on quetiapine compared to patients initiated on typical 
antipsychotics for bipolar disorder patients. Therefore, the null hypothesis was rejected and it was concluded that bipolar disorder patients initiated on typical antipsychotics incur higher mental and total healthcare costs.

\section{Conclusions from research objective 9}

For schizophrenia and bipolar disorder patients, to compare the impact of quetiapine with risperidone, olanzapine, and typical antipsychotics on components of mental healthcare costs (costs associated with mental health-related inpatient, emergency room, outpatient and pharmacy services)

The null hypothesis was that there is no difference in the impact of quetiapine compared with risperidone, olanzapine, and typical antipsychotics on components of mental healthcare costs (costs associated with mental health-related inpatient, emergency room, outpatient and pharmacy services) for schizophrenia and bipolar disorder patients. The null hypothesis was rejected for this research objective. Based on the results, we concluded that schizophrenia patients initiated on typical antipsychotics incurred slightly higher inpatient and outpatient costs but lower pharmacy costs compared to patients initiated on quetiapine. We also concluded that schizophrenia patients initiated on olanzapine incurred higher pharmacy costs compared to patients initiated on quetiapine. We also conclude that bipolar disorder patients initiated on typical antipsychotics incur slightly higher outpatient costs and lower pharmacy costs than patients who are initiated on quetiapine. Bipolar disorder patients initiated on olanzapine incur higher pharmacy costs than patients initiated on quetiapine. 


\section{Conclusions from research objective 10}

For schizophrenia and bipolar patients, to compare the impact of quetiapine with risperidone, olanzapine, and typical antipsychotics on mental health-related healthcare hospitalizations.

The null hypothesis was that there is no difference in the impact of quetiapine compared with risperidone, olanzapine, and typical antipsychotics on mental healthrelated healthcare hospitalizations for schizophrenia and bipolar disorder patients. Based on the results, we conclude that there were no significant difference in the number of mental health-related hospitalizations, proportion of patients hospitalized and time to first hospitalization among patients initiated on quetiapine and patients initiated on risperidone, olanzapine, or typical antipsychotics for both bipolar disorder and schizophrenia. However, the length of stay in hospital among schizophrenia patients initiated on typical antipsychotics was longer compared to patients initiated on quetiapine. Relevant information on hospitalization such as patient condition at the time of hospitalization and hospital discharge information were not controlled for in the analysis as they are not available in the administrative database. Therefore, we cannot conclude that there were no differences in inpatient utilization between the quetiapine and other antipsychotic cohorts. The null hypothesis was inconclusive for this objective.

\section{Conclusions from research objective 11}

For schizophrenia patients and bipolar disorder patients, to compare the impact of quetiapine with risperidone, olanzapine, and typical antipsychotics on mental healthcarerelated emergency room visits. 
The null hypothesis was that there is no difference in the impact of quetiapine compared with risperidone, olanzapine, and typical antipsychotics on mental healthcarerelated emergency room visits for schizophrenia and bipolar disorder patients. The null hypothesis was accepted and we concluded that there were no significant differences in the number of mental health-related ER visits and proportion of patients having ER visit among patients initiated on quetiapine and patients initiated on risperidone, olanzapine, or typical antipsychotics for both bipolar disorder and schizophrenia.

\section{Conclusions from research objective 12}

For schizophrenia and bipolar disorder patients, to compare the impact of quetiapine with risperidone, olanzapine, and typical antipsychotics on mental healthcare-related outpatient visits.

The null hypothesis was that there is no difference in the impact of quetiapine compared with risperidone, olanzapine, and typical antipsychotics on mental healthrelated outpatient visits for schizophrenia and bipolar disorder patients. Based on univariate analyis, outpatient visits such as psychotherapy, physician office visits and medication management visits were significantly greater among patients initiated on quetiapine compared to patients initiated on risperidone, olanzapine, and typical antipsychotics for schizophrenia and bipolar disorder patients. However, after controlling for various confounding such as patient demographics, prescribing physician type, mental health diagnosis, other medical diagnosis, type of antipsychotic, year of index antipsychotic, pre-index concomitant medication use, pre-index alcohol and substance abuse and pre-index healthcare utilization, we conclude that there was no significant 
difference in physician office visits among patients initiated on quetiapine and patients initiated on risperidone, olanzapine, or typical antipsychotics.

\section{Conclusions from research objective 13}

For schizophrenia and bipolar disorder patients, to compare the impact of quetiapine with risperidone, olanzapine, and typical antipsychotics on psychiatric medication utilization.

The null hypothesis was that there is no difference in the impact of quetiapine compared with risperidone, olanzapine, and typical antipsychotics on psychiatric medication utilization for schizophrenia and bipolar disorder patients. This null hypothesis was rejected as we found significant differences in the proportion of patients using different psychiatric drugs among patients initiated on quetiapine and patients initiated on risperidone, olanzapine, and typical antipsychotics. Among schizophrenia and bipolar disorder patients, lesser proportion of patients initiated on quetiapine used antiparkinsons drugs compared to patients initiated on typical antipsychotics. Also, larger proportion of patients initiated on quetiapine used benzodiazepine and antidepressants compared to patients initiated on typicals. Among bipolar disorder patients, greater proportion of patients initiated on risperidone used anxiolytics/ hypnotics/ sedatives compared to patients initiated on quetiapine. However, greater proportion of patients initiated on quetiapine used anxiolytics/ hypnotics/ sedatives compared to patients initiated on typicals antipsychotics. 


\section{Conclusions from research objective 14}

For schizophrenia and bipolar disorder patients, to compare the impact of quetiapine with risperidone, olanzapine, and typical antipsychotics on patient's adherence to the index medications in the post-index period.

The null hypothesis was that there is no difference in the impact of quetiapine compared with risperidone, olanzapine, and typical antipsychotics on patient's adherence to index medication for schizophrenia and bipolar disorder patients. Based on the results, we reject null hypothesis and conclude that the patients initiated on quetiapine showed similar adherence to index antipsychotics compared to patients initiated on olanzapine and risperidone but better adherence to index antipsychotics compared to patients initiated on typical antipsychotics for both schizophrenia and bipolar disorder patients.

\section{Conclusions from research objective 15}

For schizophrenia and bipolar disorder patients, to compare the impact of quetiapine with risperidone, olanzapine, and typical antipsychotics on subsequent index antipsychotic therapy modification

The null hypothesis was that there is no difference in the impact of quetiapine compared with risperidone, olanzapine, and typical antipsychotics on subsequent modification of index antipsychotic therapy by discontinuing, switching or augmenting by additional antipsychotics for schizophrenia and bipolar disorder patients. The null hypothesis was rejected and we concluded that there is significant difference in the impact of quetiapine as compared to risperidone, olanzapine and typical antipsychotics on subsequent modification of index antipsychotics. Patients initiated on typical antipsychotics and risperidone had higher hazard to modify the index antipsychotic 
therapy compared to patients initiated on quetiapine among schizophrenia patients. There are no significant differences in the hazard of index antipsychotic therapy modification among patients initiated on quetiapine compared to patients initiated on risperidone or olanzapine among bipolar patients. Bipolar patients initiated on typical antipsychotics had higher hazard to modify index antipsychotic therapy compared to patients initiated on quetiapine therapy.

\section{Limitations}

Pattern of antipsychotic use were identified based on events such as polytherapy, switching or discontinuation from index antipsychotic that occurred first after initiation of patient on index antipsychotic. However, it is possible that patients may show multiple patterns of antipsychotic utilization in course of treatment. A patient who has switched to another antipsychotic may have later discontinued antipsychotic treatment all together or switched back to index antipsychotic. As various combinations of such patterns may exist among patients, it is difficult to define, categorize and evaluate them. In addition, factors such as duration of polytherapy will also impact the costs associated with it. However, as we found significant increase in costs associated with at least one event of polytherapy, it is likely that the cost will increase further with longer duration of polytherapy. Though our study describes the pattern of antipsychotic use, it is not possible to explore all the reasons for such patterns of utilization from the information available in administrative claims data. Various factors such as physician's clinical judgment and past experience, marketing influence of pharmaceutical companies, and patient preferences can also impact these patterns. 
We have used refill records of antipsychotics to measure adherence with the therapy. This indicates that claim for that drug was paid to the provider and not that the medication was taken correctly by the patient. Information such as dates of service, amounts dispensed, and days of supply were used to calculate adherence. This is not a direct but a proxy measure of adherence. However, studies have shown significant correlations between adherence measured from refill information and other methods such as self-report, pill count, medication diary, and serum drug levels(Choo et al., 1999; Deyo, Inui, \& Sullivan, 1981). In addition, claims records are useful in calculating gaps in therapy. As Medicaid offers comprehensive coverage for prescriptions, patients in Medicaid are not very likely to fill their prescriptions outside the system. Therefore, long gaps between the refills found among the patients in our study demonstrates significant non-adherence to antipsychotic therapy in our population.

As schizophrenia and bipolar disorder are chronic illnesses, studying long term impact of drug use is important for making policy decisions. Our study follow-up period was restricted to one-year due to loss of continuously eligible patients and a change in WV Medicaid prior authorization policy for antipsychotics in the year 2003. The latter would have biased our study results. If the impact on hospitalization and ER cost is due to non-adherence or therapy discontinuation, the impact is likely to significantly increase in longer follow-ups.

The use of intent-to-treat methodology attributes all costs incurred in the followup period to the index drug. It may be argued that it is inappropriate to assign costs incurred by the patient while on another drug to the index drug. However, intent-to-treat methodology allows us to account for costs associated with patients who do not respond 
to treatment or fail treatment while comparing different treatment options. This approach is more useful to healthcare policy makers while making formulary decisions (Croghan et al., 1999).

Patients initiated on any of the typical antipsychotics during the index period were pooled together to form single cohort of typical antipsychotics. All healthcare utilization and costs incurred by these patients were aggregated and attributed to the typical antipsychotic cohort, irrespective of the type of typical antipsychotic.

Generalizability of the study is limited to populations that are similar to WV Medicaid. Factors such age, sex, race, socioeconomic status, geography, co-pays, formularies, and provider access should be taken into account before generalizing the results to a different population. Practice patterns and costs may also vary by time and introduction of new drugs.

The study is vulnerable to the limitations of using administrative claims data for research purpose (Motheral et al., 1997). Inaccurate identification of cases can seriously affect the validity of study. However, care was taken in our study to identify patients with schizophrenia and bipolar disorder based on validated algorithms for identifying this population from an administrative database (Lurie P et al., 1992; Simon et al., 1999). It is also possible that differences in costs and utilization may be due to unobserved patient differences such as disease severity. However, a study by Sernyak has validated the use of sociodemographic variables, prior utilization and cost variables obtained from administrative data as risk adjusters to predict future utilization and costs (Sernyak et al., 2003). We have statistically controlled for various confounding factors including patient 
demographics, prescribing physician type, mental health diagnosis, other medical diagnosis, type of antipsychotic, year of index antipsychotic, pre-index concomitant medication use, pre-index alcohol and substance abuse and pre-index healthcare utilization.

\section{Research Implications}

\section{Implications for payers}

Considering the rising prescription drug expenditures and adverse effects of inappropriate therapy, it is important to take steps to improve prescribing and use of prescription drugs. This is particularly imperative in the case of use of expensive antipsychotics in schizophrenia and bipolar disorder population. This patient population is known to be non-adherent to medications and suffer from alcohol and substance use disorder that further aggravates the problem. Our study found very high level of nonadherence to antipsychotic medication. The healthcare costs of non-adherent patients were higher compared to adherent patients. If payers are paying for expensive drugs, they need to be used appropriately to maximize the benefits gained from them. Our study results should encourage payers to develop policies to improve medication adherence in schizophrenia and bipolar disorder patients. Prescribing behaviors such as polytherapy and off-label use of antipsychotics should be investigated.

\section{Implications for providers}

The study findings should make the physicians aware of the extent of nonadherence to antipsychotic therapy among schizophrenia and bipolar disorder patients. The issue of non-adherence should be given attention during patient evaluation and making pharmacotherapy choice. Our study results also show significant off-label use of 
antipsychotics for mental health conditions. Patients using antipsychotics for off-label indications should be monitored closely by physicians and pharmacists for adverse effects.

\section{Direction for Future Research}

Studies with longer follow-up period are needed to capture the impact of antipsychotic non-adherence and treatment modification on healthcare costs and utilization. Future economic evaluations should include new atypical drugs such as Ziprasidone (Pfizer 2001) and Aripiprazole (Bristol Myers Squibb 2002) that could not be evaluated during the study period of this research. As schizophrenia and bipolar disorder are associated with loss of productivity, disability and caregiver burden, the impact of pharmacotherapy on productivity and societal costs should be evaluated. Studies assessing impact of antipsychotic pharmacotherapy of quality-of-life are also needed.

Our study findings reveal that a large proportion of schizophrenia and bipolar disorder patients on antipsychotics were concomitantly utilizing various psychiatric drugs such as mood stabilizers and antidepressants. Clinical trials investigating efficacy and safety of such drug combinations are necessary to assist in developing treatment guidelines for disease conditions. Future studies can also examine the role of antipsychotic monotherapy versus antipsychotic combination therapy with mood stabilizer among bipolar disorder patients. Though we found prevalence of off-label antipsychotic use among various disease conditions such as major depression, attentiondeficit disorder, autism, and others, clinical evidence supporting such use is lacking. 
Randomized clinical trials are needed to evaluate if antipsychotics offer any therapeutic advantage among patients with these disease conditions.

Reasons for switching antipsychotic and using antipsychotic polytherapy can be explored using a physician survey. Survey of physicians can also provide more information regarding outcomes associated with antipsychotic polypharmacy and offlabel antipsychotic use. There is a need for developing and evaluating behavioral interventions that can improve medication adherence among schizophrenia and bipolar disorder patients. 


\section{REFERENCES}

Treatment of schizophrenia 1999. The expert consensus guideline series (1999). J.Clin.Psychiatry, 60 Suppl 11, 3-80.

Al Zakwani, I. S., Barron, J. J., Bullano, M. F., Arcona, S., Drury, C. J., \& Cockerham, T. R. (2003). Analysis of healthcare utilization patterns and adherence in patients receiving typical and atypical antipsychotic medications. Curr.Med.Res.Opin., 19, 619-626.

Amadio, P. B., Cross, L. B., \& Amadio, P., Jr. (1997). New drugs for schizophrenia: an update for family physicians. Am.Fam.Physician, 56, 1149-1160.

American Psychiatric Association (1997). Practice guidelines for the treatment of patients with schizophrenia. Am J Psychiatry, 154 (Suppl 4).

APA (1994). Diagnostic and Statistical Manual of Mental Disorders. (4th ed ed.) Washington DC: American Psychiatric Association.

APA (1997). Practice guideline for the treatment of patients with schizophrenia. American Psychiatric Association. Am.J.Psychiatry, 154, 1-63.

Ascher-Svanum, H., Zhu, B., Faries, D., \& Ernst, F. R. (2004). A comparison of olanzapine and risperidone on the risk of psychiatric hospitalization in the naturalistic treatment of patients with schizophrenia. Ann Gen.Hosp.Psychiatry, 3, 11.

Barbee, J. G., Conrad, E. J., \& Jamhour, N. J. (2004). The effectiveness of olanzapine, risperidone, quetiapine, and ziprasidone as augmentation agents in treatmentresistant major depressive disorder. J.Clin.Psychiatry, 65, 975-981.

Bebbington, P. \& Ramana, R. (1995). The epidemiology of bipolar affective disorder. Soc.Psychiatry Psychiatr.Epidemiol., 30, 279-292.

Bogan, A. M., Shellhorn, E., Brown, E. S., McDanald, C., \& Suppes, T. (2000). Switching outpatients between atypical antipsychotics. Prog.Neuropsychopharmacol.Biol.Psychiatry, 24, 351-355.

Bottlender, R., Rudolf, D., Strauss, A., \& Moller, H. J. (2001). Mood-stabilisers reduce the risk of developing antidepressant-induced maniform states in acute treatment of bipolar I depressed patients. J.Affect.Disord., 63, 79-83.

Bowden, C. L. (2000). Efficacy of lithium in mania and maintenance therapy of bipolar disorder. J.Clin.Psychiatry, 61 Suppl 9, 35-40.

Brenner, H. D., Dencker, S. J., Goldstein, M. J., Hubbard, J. W., Keegan, D. L., Kruger, G. et al. (1990). Defining treatment refractoriness in schizophrenia.

Schizophr.Bull., 16, 551-561. 
Bright, R. A., Avorn, J., \& Everitt, D. E. (1989). Medicaid data as a resource for epidemiologic studies: strengths and limitations. J.Clin.Epidemiol., 42, 937-945.

Burns, T., Chabannes, J. P., \& Demyttenaere, K. (2002). Switching antipsychotic medications: general recommendations and switching to amisulpride. Curr.Med Res.Opin., 18, 201-208.

Byerly, M. J., Weber, M., Brooks, D., Casey, S. B., Elliot, S., \& Hawkins, J. (2003). Cost evaluation of risperidone compared with olanzapine. Psychiatr.Serv., 54, $742-744$.

Carpenter, W. T., Jr. \& Buchanan, R. W. (1994). Schizophrenia. N.Engl.J.Med, 330, 681-690.

Centorrino, F., Goren, J. L., Hennen, J., Salvatore, P., Kelleher, J. P., \& Baldessarini, R. J. (2004). Multiple versus single antipsychotic agents for hospitalized psychiatric patients: case-control study of risks versus benefits. Am.J.Psychiatry, 161, 700-706.

Choo, P. W., Rand, C. S., Inui, T. S., Lee, M. L., Cain, E., Cordeiro-Breault, M. et al. (1999). Validation of patient reports, automated pharmacy records, and pill counts with electronic monitoring of adherence to antihypertensive therapy. Med Care, 37, 846857.

Clark, R. E., Bartels, S. J., Mellman, T. A., \& Peacock, W. J. (2002). Recent trends in antipsychotic combination therapy of schizophrenia and schizoaffective disorder: implications for state mental health policy. Schizophr.Bull., 28, 75-84.

Clark, R. E., Teague, G. B., Ricketts, S. K., Bush, P. W., Xie, H., McGuire, T. G. et al. (1998). Cost-effectiveness of assertive community treatment versus standard case management for persons with co-occurring severe mental illness and substance use disorders. Health Serv.Res., 33, 1285-1308.

Cooper, W. O., Hickson, G. B., Fuchs, C., Arbogast, P. G., \& Ray, W. A. (2004). New Users of Antipsychotic Medications Among Children Enrolled in TennCare. Arch.Pediatr.Adolesc.Med, 158, 753-759.

Craig, T. J., Grossman, S., Mojtabai, R., Gibson, P. J., Lavelle, J., Carlson, G. A. et al. (2004). Medication use patterns and 2-year outcome in first-admission bipolar disorder with psychotic features. Bipolar.Disord., 6, 406-415.

Crismon ML \& Dorson PG. (1997). Schizophrenia. In DiPiro JT, Talbert RL, \& Yee GC (Eds.), Pharmacotherapy: A Pathophysiologic Approach (3rd ed., pp. 13671394). Stamford, Conn: Appleton \& Lange.

Croghan, T. W., Johnstone, B. M., Buesching, D. P., \& Kessler, R. C. (1999). Information needs for medication coverage decisions in a state Medicaid program. Med.Care, 37, AS24-AS31. 
Crown, W. H., Obenchain, R. L., Englehart, L., Lair, T., Buesching, D. P., \& Croghan, T. (1998). The application of sample selection models to outcomes research: the case of evaluating the effects of antidepressant therapy on resource utilization. Stat.Med., 17, 1943-1958.

Crown, W. H., Treglia, M., Meneades, L., \& White, A. (2001). Long-term costs of treatment for depression: impact of drug selection and guideline adherence. Value.Health, 4, 295-307.

Csernansky, J. G. \& Schuchart, E. K. (2002). Relapse and rehospitalisation rates in patients with schizophrenia: effects of second generation antipsychotics. CNS.Drugs, $16,473-484$.

Deyo, R. A., Cherkin, D. C., \& Ciol, M. A. (1992). Adapting a clinical comorbidity index for use with ICD-9-CM administrative databases. J.Clin.Epidemiol., 45, 613-619.

Deyo, R. A., Inui, T. S., \& Sullivan, B. (1981). Noncompliance with arthritis drugs: magnitude, correlates, and clinical implications. J Rheumatol., 8, 931-936.

Dolder, C. R., Lacro, J. P., Dunn, L. B., \& Jeste, D. V. (2002). Antipsychotic medication adherence: is there a difference between typical and atypical agents? Am.J.Psychiatry, 159, 103-108.

Eaddy, M., Grogg, A., \& Locklear, J. (2005). Assessment of compliance with antipsychotic treatment and resource utilization in a medicaid population. Clin.Ther., 27, 263-272.

Edgell, E. T., Andersen, S. W., Johnstone, B. M., Dulisse, B., Revicki, D., \& Breier, A. (2000). Olanzapine versus risperidone. A prospective comparison of clinical and economic outcomes in schizophrenia. Pharmacoeconomics., 18, 567-579.

Emsley, R. A., Raniwalla, J., Bailey, P. J., \& Jones, A. M. (2000). A comparison of the effects of quetiapine ('seroquel') and haloperidol in schizophrenic patients with a history of and a demonstrated, partial response to conventional antipsychotic treatment. PRIZE Study Group. Int.Clin.Psychopharmacol., 15, 121-131.

Foster, R. H. \& Goa, K. L. (1998). Risperidone. A pharmacoeconomic review of its use in schizophrenia. Pharmacoeconomics., 14, 97-133.

Fountoulakis, K. N., Vieta, E., Sanchez-Moreno, J., Kaprinis, S. G., Goikolea, J. M., \& Kaprinis, G. S. (2005). Treatment guidelines for bipolar disorder: A critical review. J.Affect.Disord., 86, 1-10.

Freudenreich, O. \& Goff, D. C. (2002). Antipsychotic combination therapy in schizophrenia. A review of efficacy and risks of current combinations. Acta Psychiatr.Scand., 106, 323-330. 
Frye, M. A., Ketter, T. A., Altshuler, L. L., Denicoff, K., Dunn, R. T., Kimbrell, T. A. et al. (1998). Clozapine in bipolar disorder: treatment implications for other atypical antipsychotics. J.Affect.Disord., 48, 91-104.

Fuller, M. A., Shermock, K. M., Secic, M., Laich, J. S., \& Durkin, M. B. (2002). Service use and costs among VA patients with schizophrenia taking risperidone or olanzapine. Psychiatr.Serv., 53, 855-860.

Ganguly, R., Kotzan, J. A., Miller, L. S., Kennedy, K., \& Martin, B. C. (2004). Prevalence, trends, and factors associated with antipsychotic polypharmacy among Medicaid-eligible schizophrenia patients, 1998-2000. J.Clin.Psychiatry, 65, 1377-1388.

Gianfrancesco, F., Durkin, M. B., Mahmoud, R., \& Wang, R. H. (2002). Use of healthcare services by patients treated with risperidone versus conventional antipsychotic agents. Pharmacoeconomics., 20, 413-427.

Gianfrancesco, F., Pesa, J., \& Wang, R. H. (2005). Comparison of mental health resources used by patients with bipolar disorder treated with risperidone, olanzapine, or quetiapine. J.Manag.Care Pharm., 11, 220-230.

Gibson, P. J., Damler, R., Jackson, E. A., Wilder, T., \& Ramsey, J. L. (2004). The impact of olanzapine, risperidone, or haloperidol on the cost of schizophrenia care in a medicaid population. Value.Health, 7, 22-35.

Gilmer, T. P., Dolder, C. R., Lacro, J. P., Folsom, D. P., Lindamer, L., Garcia, P. et al. (2004). Adherence to treatment with antipsychotic medication and health care costs among Medicaid beneficiaries with schizophrenia. Am.J.Psychiatry, 161, 692-699.

Goldberg, J. F., Harrow, M., \& Grossman, L. S. (1995). Course and outcome in bipolar affective disorder: a longitudinal follow-up study. Am.J.Psychiatry, 152, 379-384.

Goldner, E. M., Jones, W., \& Waraich, P. (2003). Using Administrative Data to Analyze the Prevalence and Distribution of Schizophrenic Disorders. Psychiatric Services, 54, 1017-1021.

Greene, W. H. (2000). Econometric analysis . (4th ed.) Upper Saddle River, NJ: Prentice Hall.

Hamner, M. B., Arvanitis, L. A., Miller, B. G., Link, C. G., \& Hong, W. W. (1996). Plasma prolactin in schizophrenia subjects treated with Seroquel (ICI 204,636). Psychopharmacol.Bull., 32, 107-110.

Harrow, M., Goldberg, J. F., Grossman, L. S., \& Meltzer, H. Y. (1990). Outcome in manic disorders. A naturalistic follow-up study. Arch.Gen.Psychiatry, 47, 665-671.

Haywood, T. W., Kravitz, H. M., Grossman, L. S., Cavanaugh, J. L., Jr., Davis, J. M., \& Lewis, D. A. (1995). Predicting the "revolving door" phenomenon among patients 
with schizophrenic, schizoaffective, and affective disorders. Am.J.Psychiatry, 152, 856861.

Heckman, J. J. (1979). Sample selection bias as a specification error. Econometrica, 47, 153-161.

Hilty, D. M., Brady, K. T., \& Hales, R. E. (1999). A review of bipolar disorder among adults. Psychiatr.Serv., 50, 201-213.

Hirschfeld RMA, Calabrese JR, \& Weissman M (2005). Lifetime prevalence of bipolar I and II disorders in the United States. Abstract presented at: The 155th Annual Meeting of the American Psychiatric Association; May 18-23, 2002; Philadelphia, PA. In.

Hogan, M. F. (1998). The public sector and mental health parity: time for inclusion. J.Ment.Health Policy Econ., 1, 189-198.

Hogan, M. F. (1999). Public-sector mental health care: new challenges. Health Aff.(Millwood.), 18, 106-111.

Hopkins, H. S. \& Gelenberg, A. J. (1994). Treatment of bipolar disorder: how far have we come? Psychopharmacol.Bull., 30, 27-38.

Hudson, T. J., Owen, R. R., Thrush, C. R., Han, X., Pyne, J. M., Thapa, P. et al. (2004). A pilot study of barriers to medication adherence in schizophrenia.

J.Clin.Psychiatry, 65, 211-216.

Hunt, G. E., Bergen, J., \& Bashir, M. (2002). Medication compliance and comorbid substance abuse in schizophrenia: impact on community survival 4 years after a relapse. Schizophr.Res., 54, 253-264.

Jenkins, R. (1997). Reducing the burden of mental illness. Lancet, 349, 1340.

Jerrell, J. M. (2002). Cost-effectiveness of risperidone, olanzapine, and conventional antipsychotic medications. Schizophr.Bull., 28, 589-605.

Kamali, M., Kelly, L., Gervin, M., Browne, S., Larkin, C., \& O'Callaghan, E. (2001). Psychopharmacology: insight and comorbid substance misuse and medication compliance among patients with schizophrenia. Psychiatr.Serv., 52, 161-3, 166.

Kaplan, M. (2000). Atypical antipsychotics for treatment of mixed depression and anxiety. J.Clin.Psychiatry, 61, 388-389.

Kashner, T. M., Rader, L. E., Rodell, D. E., Beck, C. M., Rodell, L. R., \& Muller, K. (1991). Family characteristics, substance abuse, and hospitalization patterns of patients with schizophrenia. Hosp.Community Psychiatry, 42, 195-196. 
Kasper, S., Jones, M., \& Duchesne, I. (2001). Risperidone olanzapine drug outcomes studies in schizophrenia (RODOS): health economic results of an international naturalistic study. Int.Clin.Psychopharmacol., 16, 189-196.

Keck, P. E., Jr., McElroy, S. L., Strakowski, S. M., Bourne, M. L., \& West, S. A. (1997). Compliance with maintenance treatment in bipolar disorder. Psychopharmacol.Bull., 33, 87-91.

Kelly, D. L., Nelson, M. W., Love, R. C., Yu, Y., \& Conley, R. R. (2001). Comparison of discharge rates and drug costs for patients with schizophrenia treated with risperidone or olanzapine. Psychiatr.Serv., 52, 676-678.

Kessler, R. C., McGonagle, K. A., Zhao, S., Nelson, C. B., Hughes, M., Eshleman, S. et al. (1994). Lifetime and 12-month prevalence of DSM-III-R psychiatric disorders in the United States. Results from the National Comorbidity Survey. Arch.Gen.Psychiatry, 51, 8-19.

Knapp, M. (1997). Costs of schizophrenia. Br.J.Psychiatry, 171, 509-518.

Knapp, M., King, D., Pugner, K., \& Lapuerta, P. (2004). Non-adherence to antipsychotic medication regimens: associations with resource use and costs. Br.J.Psychiatry, 184, 509-516.

Kogut, S. J., Yam, F., \& Dufresne, R. (2005). Prescribing of antipsychotic medication in a medicaid population: use of polytherapy and off-label dosages. J.Manag.Care Pharm., 11, 17-24.

Koro, C. E., Fedder, D. O., L'Italien, G. J., Weiss, S. S., Magder, L. S., Kreyenbuhl, J. et al. (2002). Assessment of independent effect of olanzapine and risperidone on risk of diabetes among patients with schizophrenia: population based nested case-control study. BMJ, 325, 243.

Lacro, J. P., Dunn, L. B., Dolder, C. R., Leckband, S. G., \& Jeste, D. V. (2002). Prevalence of and risk factors for medication nonadherence in patients with schizophrenia: a comprehensive review of recent literature. J.Clin.Psychiatry, 63, 892909.

Lehman, A. F., Kreyenbuhl, J., Buchanan, R. W., Dickerson, F. B., Dixon, L. B., Goldberg, R. et al. (2004a). The Schizophrenia Patient Outcomes Research Team (PORT): updated treatment recommendations 2003. Schizophr.Bull., 30, 193-217.

Lehman, A. F., Lieberman, J. A., Dixon, L. B., McGlashan, T. H., Miller, A. L., Perkins, D. O. et al. (2004c). Practice guideline for the treatment of patients with schizophrenia, second edition. Am.J.Psychiatry, 161, 1-56.

Lehman, A. F., Lieberman, J. A., Dixon, L. B., McGlashan, T. H., Miller, A. L., Perkins, D. O. et al. (2004b). Practice guideline for the treatment of patients with schizophrenia, second edition. Am.J.Psychiatry, 161, 1-56. 
Lehman, A. F. \& Steinwachs, D. M. (1998). Patterns of usual care for schizophrenia: initial results from the Schizophrenia Patient Outcomes Research Team (PORT) Client Survey. Schizophr.Bull., 24, 11-20.

Leslie, D. L. \& Rosenheck, R. A. (2001). Use of pharmacy data to assess quality of pharmacotherapy for schizophrenia in a national health care system: individual and facility predictors. Med.Care, 39, 923-933.

Levine, J., Chengappa, K. N., Brar, J. S., Gershon, S., \& Kupfer, D. J. (2001). Illness characteristics and their association with prescription patterns for bipolar I disorder. Bipolar.Disord., 3, 41-49.

Lewinsohn, P. M., Klein, D. N., \& Seeley, J. R. (1995). Bipolar disorders in a community sample of older adolescents: prevalence, phenomenology, comorbidity, and course. J.Am.Acad.Child Adolesc.Psychiatry, 34, 454-463.

Licht, R. W., Gouliaev, G., Vestergaard, P., Dybbro, J., Lund, H., \& Merinder, L. (1994). Treatment of manic episodes in Scandinavia: the use of neuroleptic drugs in a clinical routine setting. J.Affect.Disord., 32, 179-185.

Lindenmayer, J. P., Czobor, P., Volavka, J., Citrome, L., Sheitman, B., McEvoy, J. P. et al. (2003). Changes in glucose and cholesterol levels in patients with schizophrenia treated with typical or atypical antipsychotics. Am.J.Psychiatry, 160, 290296.

Liperoti, R., Mor, V., Lapane, K. L., Pedone, C., Gambassi, G., \& Bernabei, R. (2003). The use of atypical antipsychotics in nursing homes. J.Clin.Psychiatry, 64, 11061112 .

Lish, J. D., Dime-Meenan, S., Whybrow, P. C., Price, R. A., \& Hirschfeld, R. M. (1994). The National Depressive and Manic-depressive Association (DMDA) survey of bipolar members. J.Affect.Disord., 31, 281-294.

Loosbrock, D. L., Zhao, Z., Johnstone, B. M., \& Morris, L. S. (2003). Antipsychotic medication use patterns and associated costs of care for individuals with schizophrenia. J.Ment.Health Policy Econ., 6, 67-75.

Lurie P, Popkin M, Dysken M, \& Finch M. (1992). Accuracy of Diagnosis of Schizophrenia in Medicaid Claims. Hospital and Community Psychiatry, 43, 69-71.

Maarbjerg, K., Aagaard, J., \& Vestergaard, P. (1988). Adherence to lithium prophylaxis: I. Clinical predictors and patient's reasons for nonadherence. Pharmacopsychiatry, 21, 121-125.

Mark, T. L. \& Coffey, R. M. (2003). What drove private health insurance spending on mental health and substance abuse care, 1992-1999? Health Aff.(Millwood.), $22,165-172$. 
Mauskopf, J. A., David, K., Grainger, D. L., \& Gibson, P. J. (1999). Annual health outcomes and treatment costs for schizophrenia populations. J.Clin.Psychiatry, 60 Suppl 19, 14-19.

McCollam, P. L., Lage, M. J., \& Bala, M. (2001). A comparison of total hospital costs for percutaneous coronary intervention patients receiving abciximab versus tirofiban. Catheter.Cardiovasc.Interv., 54, 152-157.

McCombs, J. S., Nichol, M. B., Johnstone, B. M., Stimmel, G. L., Shi, J., \& Smith, R. (2000). Antipsychotic drug use patterns and the cost of treating schizophrenia. Psychiatr.Serv., 51, 525-527.

McCombs, J. S., Nichol, M. B., Stimmel, G. L., Shi, J., \& Smith, R. R. (1999a). Use patterns for antipsychotic medications in medicaid patients with schizophrenia. J.Clin.Psychiatry, 60 Suppl 19, 5-11.

McCombs, J. S., Nichol, M. B., Stimmel, G. L., Shi, J., \& Smith, R. R. (1999b). Use patterns for antipsychotic medications in medicaid patients with schizophrenia. J.Clin.Psychiatry, 60 Suppl 19, 5-11.

McCue, R. E., Waheed, R., \& Urcuyo, L. (2003). Polypharmacy in patients with schizophrenia. J.Clin.Psychiatry, 64, 984-989.

McElroy, S. L., Keck, P. E., Jr., \& Strakowski, S. M. (1996). Mania, psychosis, and antipsychotics. J.Clin.Psychiatry, 57 Suppl 3, 14-26.

Menzin, J., Boulanger, L., Friedman, M., Mackell, J., \& Lloyd, J. R. (2003). Treatment adherence associated with conventional and atypical antipsychotics in a large state Medicaid program. Psychiatr.Serv., 54, 719-723.

Miller, A. L. (2004). PORT treatment recommendations. Schizophr.Bull., 30, 601604.

Miller, A. L., Chiles, J. A., Chiles, J. K., Crismon, M. L., Rush, A. J., \& Shon, S. P. (1999). The Texas Medication Algorithm Project (TMAP) schizophrenia algorithms. J.Clin.Psychiatry, 60, 649-657.

Mladsi, D. M., Grogg, A. L., Irish, W. D., Lopez, R. B., Degen, K., Swann, A. et al. (2004). Pharmacy cost evaluation of risperidone, olanzapine, and quetiapine for the treatment of schizophrenia in acute care inpatient settings. Curr.Med.Res.Opin., 20, 1883-1893.

Mojtabai, R., Lavelle, J., Gibson, P. J., Sohler, N. L., Craig, T. J., Carlson, G. A. et al. (2002). Gaps in use of antipsychotics after discharge by first-admission patients with schizophrenia, 1989 to 1996. Psychiatr.Serv., 53, 337-339.

Moller, H. J. \& Nasrallah, H. A. (2003). Treatment of bipolar disorder. J.Clin.Psychiatry, 64 Suppl 6, 9-17. 
Mortimer, A., Williams, P., \& Meddis, D. (2003). Impact of side-effects of atypical antipsychotics on non-compliance, relapse and cost. J.Int.Med Res., 31, 188-196.

Motheral, B. R. \& Fairman, K. A. (1997). The use of claims databases for outcomes research: rationale, challenges, and strategies. Clin.Ther., 19, 346-366.

Namjoshi, M. A., Rajamannar, G., Jacobs, T., Sanger, T. M., Risser, R., Tohen, M. F. et al. (2002). Economic, clinical, and quality-of-life outcomes associated with olanzapine treatment in mania. Results from a randomized controlled trial. J.Affect.Disord., 69, 109-118.

Narrow, W. E., Regier, D. A., Rae, D. S., Manderscheid, R. W., \& Locke, B. Z. (1993). Use of services by persons with mental and addictive disorders. Findings from the National Institute of Mental Health Epidemiologic Catchment Area Program. Arch.Gen.Psychiatry, 50, 95-107.

Nightengale, B. S., Garrett, L., Waugh, S., Lawrence, B. J., \& Andrus, J. (1998). Economic outcomes associated with the use of risperidone in a naturalistic group practice setting. Am.J.Manag.Care, 4, 360-366.

O'Connell, R. A., Mayo, J. A., Flatow, L., Cuthbertson, B., \& O'Brien, B. E. (1991). Outcome of bipolar disorder on long-term treatment with lithium. Br.J.Psychiatry, 159, 123-129.

Ostroff, R. B. \& Nelson, J. C. (1999). Risperidone augmentation of selective serotonin reuptake inhibitors in major depression. J.Clin.Psychiatry, 60, 256-259.

Perlis, R. H. (2005). The role of pharmacologic treatment guidelines for bipolar disorder. J.Clin.Psychiatry, 66 Suppl 3:37-47., 37-47.

Perry, A., Tarrier, N., Morriss, R., McCarthy, E., \& Limb, K. (1999). Randomised controlled trial of efficacy of teaching patients with bipolar disorder to identify early symptoms of relapse and obtain treatment. $B M J, 318,149-153$.

Pigott, T. A., Carson, W. H., Saha, A. R., Torbeyns, A. F., Stock, E. G., \& Ingenito, G. G. (2003). Aripiprazole for the prevention of relapse in stabilized patients with chronic schizophrenia: a placebo-controlled 26-week study. J.Clin.Psychiatry, 64, 1048-1056.

Procyshyn, R. M., Kennedy, N. B., Tse, G., \& Thompson, B. (2001). Antipsychotic polypharmacy: a survey of discharge prescriptions from a tertiary care psychiatric institution. Can.J.Psychiatry, 46, 334-339.

Procyshyn, R. M. \& Thompson, B. (2004). Patterns of antipsychotic utilization in a tertiary care psychiatric institution. Pharmacopsychiatry, 37, 12-17. 
Procyshyn, R. M. \& Zerjav, S. (1998). Drug utilization patterns and outcomes associated with in-hospital treatment with risperidone or olanzapine. Clin.Ther., 20, 1203-1217.

Rascati, K. L., Johnsrud, M. T., Crismon, M. L., Lage, M. J., \& Barber, B. L. (2003). Olanzapine versus risperidone in the treatment of schizophrenia : a comparison of costs among Texas Medicaid recipients. Pharmacoeconomics., 21, 683-697.

Regier, D. A., Narrow, W. E., Rae, D. S., Manderscheid, R. W., Locke, B. Z., \& Goodwin, F. K. (1993). The de facto US mental and addictive disorders service system. Epidemiologic catchment area prospective 1-year prevalence rates of disorders and services. Arch.Gen.Psychiatry, 50, 85-94.

Revicki, D. A. (1997). Methods of pharmacoeconomic evaluation of psychopharmacologic therapies for patients with schizophrenia. J.Psychiatry Neurosci., 22, 256-266.

Revicki, D. A., Paramore, L. C., Sommerville, K. W., Swann, A. C., \& Zajecka, J. M. (2003). Divalproex sodium versus olanzapine in the treatment of acute mania in bipolar disorder: health-related quality of life and medical cost outcomes.

J.Clin.Psychiatry, 64, 288-294.

Rural Urban Continuum codes (2003). Available

at:(http://www.ers.usda.gov/Data/RuralUrbanContinuumCodes/) Accessed on 01/20/2005 [On-line].

Russo, P., Smith, M. W., Dirani, R., Namjoshi, M., \& Tohen, M. (2002).

Pharmacotherapy patterns in the treatment of bipolar disorder. Bipolar.Disord., 4, 366377.

Sachs, G. S., Printz, D. J., Kahn, D. A., Carpenter, D., \& Docherty, J. P. (2000). The Expert Consensus Guideline Series: Medication Treatment of Bipolar Disorder 2000. Postgrad.Med, Spec No, 1-104.

Salloum, I. M. \& Thase, M. E. (2000). Impact of substance abuse on the course and treatment of bipolar disorder. Bipolar.Disord., 2, 269-280.

Sanger, T. M., Tohen, M., Vieta, E., Dunner, D. L., Bowden, C. L., Calabrese, J. R. et al. (2003). Olanzapine in the acute treatment of bipolar I disorder with a history of rapid cycling. J.Affect.Disord., 73, 155-161.

Schou, M. (1997). The combat of non-compliance during prophylactic lithium treatment. Acta Psychiatr.Scand., 95, 361-363.

Schumacher, J. E., Makela, E. H., \& Griffin, H. R. (2003). Multiple antipsychotic medication prescribing patterns. Ann Pharmacother., 37, 951-955. 
Schumann, C., Lenz, G., Berghofer, A., \& Muller-Oerlinghausen, B. (1999). Nonadherence with long-term prophylaxis: a 6-year naturalistic follow-up study of affectively ill patients. Psychiatry Res., 89, 247-257.

Schwartz, A. H., Perlman, B. B., Paris, M., Schmidt, K., \& Thornton, J. C. (1980). Psychiatric diagnoses as reported to Medicaid and as recorded in patient charts. Am.J.Public Health, 70, 406-408.

Scott, J. (1993). Homelessness and mental illness. Br.J.Psychiatry, 162, 314-324.

Scott, J. \& Pope, M. (2002). Self-reported adherence to treatment with mood stabilizers, plasma levels, and psychiatric hospitalization. Am.J.Psychiatry, 159, 1927 1929.

Sernyak, M. J. \& Rosenheck, R. (2003). Risk adjustment in studies using administrative data. Schizophr.Bull., 29, 267-271.

Sernyak, M. J. \& Rosenheck, R. (2004). Clinicians' reasons for antipsychotic coprescribing. J.Clin.Psychiatry, 65, 1597-1600.

Shattell, M. \& Keltner, N. L. (2004). The case for atypical antipsychotics in bipolar disorder. Perspect.Psychiatr.Care, 40, 34-38.

Shelton, R. C. (2003). The combination of olanzapine and fluoxetine in mood disorders. Expert.Opin.Pharmacother., 4, 1175-1183.

Shepherd, G. \& Hill, R. G. (1996). Manic depression: do people receive adequate support? Nurs.Times, 92, 42-44.

Simon, G. E. \& Unutzer, J. (1999). Health care utilization and costs among patients treated for bipolar disorder in an insured population. Psychiatr.Serv., 50, 13031308.

Solomon, D. A., Keitner, G. I., Miller, I. W., Shea, M. T., \& Keller, M. B. (1995). Course of illness and maintenance treatments for patients with bipolar disorder.

J.Clin.Psychiatry, 56, 5-13.

Strakowski, S. M., Del Bello, M. P., Adler, C. M., \& Keck, P. E., Jr. (2003). Atypical antipsychotics in the treatment of bipolar disorder. Expert.Opin.Pharmacother., $4,751-760$.

Strakowski, S. M. \& DelBello, M. P. (2000). The co-occurrence of bipolar and substance use disorders. Clin.Psychol.Rev., 20, 191-206.

Strakowski, S. M., Sax, K. W., McElroy, S. L., Keck, P. E., Jr., Hawkins, J. M., \& West, S. A. (1998). Course of psychiatric and substance abuse syndromes co-occurring with bipolar disorder after a first psychiatric hospitalization. J.Clin.Psychiatry, 59, 465471. 
Strom, B. (2005). Pharmacoepidemiology. (Third ed.) New York: Wiley and Sons.

Suppes, T., Baldessarini, R. J., Faedda, G. L., \& Tohen, M. (1991). Risk of recurrence following discontinuation of lithium treatment in bipolar disorder. Arch.Gen.Psychiatry, 48, 1082-1088.

Suppes, T., Rush, A. J., Dennehy, E. B., Crismon, M. L., Kashner, T. M., Toprac, M. G. et al. (2003). Texas Medication Algorithm Project, phase 3 (TMAP-3): clinical results for patients with a history of mania. J.Clin.Psychiatry, 64, 370-382.

Tapp, A., Wood, A. E., Secrest, L., Erdmann, J., Cubberley, L., \& Kilzieh, N. (2003). Combination antipsychotic therapy in clinical practice. Psychiatr.Serv., 54, 5559.

Tempier, R. P. \& Pawliuk, N. H. (2003). Conventional, atypical, and combination antipsychotic prescriptions: a 2-year comparison. J.Clin.Psychiatry, 64, 673-679.

Tilden, D., Aristides, M., Meddis, D., \& Burns, T. (2002). An economic assessment of quetiapine and haloperidol in patients with schizophrenia only partially responsive to conventional antipsychotics. Clin.Ther., 24, 1648-1667.

Tohen, M. \& Zarate, C. A., Jr. (1998). Antipsychotic agents and bipolar disorder. J.Clin.Psychiatry, 59 Suppl 1, 38-48.

Tohen, M., Zhang, F., Taylor, C. C., Burns, P., Zarate, C., Sanger, T. et al. (2001). A meta-analysis of the use of typical antipsychotic agents in bipolar disorder. J.Affect.Disord., 65, 85-93.

Tse, S. S. \& Walsh, A. E. (2001). How does work work for people with bipolar affective disorder? Occup.Ther.Int., 8, 210-225.

Van Putten, T. (1974). Why do schizophrenic patients refuse to take their drugs? Arch.Gen.Psychiatry, 31, 67-72.

Voris, J. C. \& Glazer, W. M. (1999). Use of risperidone and olanzapine in outpatient clinics at six Veterans Affairs hospitals. Psychiatr.Serv., 50, 163-4, 168.

Waddington, J. L., Youssef, H. A., \& Kinsella, A. (1998). Mortality in schizophrenia. Antipsychotic polypharmacy and absence of adjunctive anticholinergics over the course of a 10-year prospective study. Br.J.Psychiatry, 173, 325-329.

Weiden, P., Aquila, R., \& Standard, J. (1996). Atypical antipsychotic drugs and long-term outcome in schizophrenia. J.Clin.Psychiatry, 57 Suppl 11, 53-60.

Weiden, P. J., Kozma, C., Grogg, A., \& Locklear, J. (2004). Partial compliance and risk of rehospitalization among California Medicaid patients with schizophrenia. Psychiatr.Serv., 55, 886-891. 
Weiden, P. J. \& Olfson, M. (1995). Cost of relapse in schizophrenia. Schizophr.Bull., 21, 419-429.

Weissman, M. M., Bland, R. C., Canino, G. J., Faravelli, C., Greenwald, S., Hwu, H. G. et al. (1996). Cross-national epidemiology of major depression and bipolar disorder. JAMA, 276, 293-299.

Weissman, M. M., Leaf, P. J., Tischler, G. L., Blazer, D. G., Karno, M., Bruce, M. L. et al. (1988). Affective disorders in five United States communities. Psychol.Med, $18,141-153$.

Williams, C. L., Johnstone, B. M., Kesterson, J. G., Javor, K. A., \& Schmetzer, A. D. (1999). Evaluation of antipsychotic and concomitant medication use patterns in patients with schizophrenia. Med.Care, 37, AS81-AS86.

Wyatt, R. J. \& Henter, I. (1995). An economic evaluation of manic-depressive illness--1991. Soc.Psychiatry Psychiatr.Epidemiol., 30, 213-219.

Zhao, Z. (2002). A retrospective economic evaluation of olanzapine versus risperidone in the treatment of schizophrenia. Manag. Care Interface, 15, 75-81.

Zhao, Z. (2004). Economic outcomes associated with olanzapine versus risperidone in the treatment of uncontrolled schizophrenia. Curr.Med.Res.Opin., 20, 1039-1048. 


\title{
CURRICULUM VITAE
}

\author{
Mariam K. Hassan
}

E-mail: $\underline{\text { mhassan@hsc.wvu.edu }}$

\section{EDUCATION}

August 1999-July 2005

Doctor of Philosophy (Ph.D.), Dept. of Pharmaceutical Systems \& Policy, West

Virginia University

Dissertation: Evaluating Medication Utilization Patterns and Healthcare

Outcomes in Patients receiving Antipsychotics

June 1994-June 1998

Bachelor of Pharmacy (B. Pharm), University of Mumbai, India

\section{RESEARCH AND PROFESSIONAL EXPERIENCE}

December 2004-Present

Principal Investigator

Research Grant funded by AstraZeneca, for the project "Economic Evaluation of Antipsychotics.” Total Funding: \$54,000.00, Grant/ Contract \#: 1002887R

October 2003-November 2003

Research Assistant

Assisted on a report reviewing various Pharmacy Benefit Management (PBM) strategies for the West Virginia Worker's Compensation Commission (WVWCC)

May 2003-August 2003

\section{Summer Intern}

\section{Health Benchmarks, Inc}

- Conducted a retrospective claims data analysis comparing utilization, cost and adherence between dual and single controller asthma medications using advanced regression models and survival analysis (Study sponsored by GlaxoSmithKline)

- Carried out a cost-benefit analysis of chiropractic insurance coverage in a managed care network using techniques such as sample selection models and two-part models (Study sponsored by American Specialty Health)

- Patient-reported outcomes analysis for REACH (Registry for the Enhancement of Asthma Control and Health)

Aug 2002-December 2004

\section{Research Assistant}

Examine utilization characteristics of narcotic analgesics in the WV Worker's Compensation system for a project funded by the WV Bureau of Employment Programs. Developed the study design and data analysis method to identify 
overuse/inappropriate use of narcotic analgesics and evaluate their cost and utilization

Aug 2002-December 2003

\section{Research Assistant}

Survey of West Virginia Medicaid beneficiaries to assess the prevalence of tobacco use \& need for smoking cessation programs for a study funded by West Virginia Bureau of Medical Services. Assisted in data analysis and preparing manuscripts

Aug 2001-December 2004

Research Assistant, School of Medicine, West Virginia University

Responsibilities include IRB application process, research design, literature review, data analysis, SAS programming, preparing reports, manuscripts and presentations as well as providing consultations for student practicum projects for the Masters in Public Health (MPH) program

Aug 2001-December 2004

Research Assistant

Development and management of prospective epidemiological database for studies on occupational and environmental hazards. The project was funded by Institute of Occupational and Environmental Health (IOEH) and Association of Occupational and Environmental Clinics (AOEC).

Responsibilities include IRB application process, developing physician survey, and patient data collection

Aug 1999-July 2001

Teaching Assistant, School of Pharmacy, West Virginia University

Responsibilities include lecturing, grading, management of course material, and facilitation of student projects

July 1998-July 1999

Research Apprentice, $R$ \& $D$ Formulations, Elder Pharmaceutical Limited, Bombay, India

Responsibilities included pre-formulation and formulation studies of antispasmodic agent - Oxybutynin chloride (Nocturin $\left.{ }^{\circledR}\right)$, dosage form design, supervision of "Product Development Trial Batches" of tablets and syrups, coordinated meetings between formulation development and marketing

\section{PUBLICATIONS}

Hassan M and Amonkar MM. "Aspirin Use for Primary and Secondary Prophylaxis of Cardiovascular Disease." Current Therapeutic Research.2001; 62:676-690.

Hassan M, Joshi A, Madhavan S, and Amonkar M. "Obesity and Health-Related Quality of Life: A Cross-sectional Analysis of US population." International Journal of Obesity 2003; 27(10):1227-32. 
Scobbo RR, Vondohlen TW, Hassan M and Islam S. "Serum TSH Variability in Normal Individuals: Influence of Time of Sample Collection." West Virginia Medical Journal. 2004; 100 (July/August): 138-142.

Hassan M and Miller L. Compliance Therapy in Schizophrenia: Addressing Possible Reasons for No Significant Differences and the Need to Adjust for Antipsychotic Type. (Letter to Editor) British Medical Journal 2003; 327: 834-0. Available at: http://bmj.bmjjournals.com/cgi/eletters/327/7419/834\#39684.

Hassan M, Kalsekar I, Madhavan S, Mody R and Amonkar M. Determinants of Readiness to Quit Smoking among Women of Child Bearing Age. (Under review) Women's Health Issues.

\section{PRESENTATIONS}

Hassan M, Kavookjian J, Madhavan S. "Study gaps in economic evaluations of pharmacotherapy in bipolar disorder." Poster presentation, Tenth Annual International Meeting of International Society for Pharmacoeconomic and Outcome Research (ISPOR), May 15-18, 2005, Washington DC.

Islam S, Hassan M, Doyle E. Becker J, Weikle P, Ducatman A. "Quantification of Suspected Addiction Treatment of Narcotic Analgesics using Prescription Sequence Analysis: Experience of a State-Based Worker's Compensation System." Poster presentation, International Society for Pharmacoeconomic and Outcome Research (ISPOR) 7th Annual European Congress, October 24-26, 2004, Hamburg, Germany.

Legoretta A, Hassan M. "Prevalence of Antidepressant Use in Children and Adolescents: Who Prescribes and What are the Diagnoses." Poster presentation, Annual Meeting of the American Pharmaceutical Association (APhA), March 2630, 2004, Seattle, Washington.

Hassan M, Islam S, Doyle E, Ducatman A. "Utilization Characteristics of Narcotic Analgesics in WV Workers' Compensation Claimants." Poster Presentation, Eight Annual International Meeting of International Society for Pharmacoeconomic and Outcome Research (ISPOR), May 18-21, 2003, Arlington, Virginia.

Hassan M, Amonkar M. "Resource Utilization for Inpatient Asthma Care in Children and Adults: An analysis of HCUP data." Poster Presentation, Seventh Annual International Meeting of International Society for Pharmacoeconomic and Outcome Research (ISPOR), May 19-22, 2002, Arlington, Virginia.

Wierman T, Slain D, Hassan M, Miller K, Amonkar M. “A Benchmarking Survey Study on Lipid-Based Amphotericin B Usage at Various U.S Institutions." Poster Presentation, $36^{\text {th }}$ Annual American Society of Health-System Pharmacists (ASHP) Midyear Clinical Meeting, December 2-6, 2001, New Orleans, Louisiana. 
Hassan M, Kamal K, Mody R, Amonkar M. "Breast and Cervical Cancer Screening among Obese Women in the United States." Poster Presentation, $129^{\text {th }}$ Annual Meeting of American Public Health Association (APHA), October 21-25, 2001, Atlanta, Georgia.

Hassan M, Joshi A, Madhavan S, Amonkar M. "Impact of Obesity on HealthRelated Quality of Life: An Analysis of BRFSS Data." Poster presentation, Sixth Annual International Meeting of International Society for Pharmacoeconomic and Outcome Research (ISPOR), May 20-23, 2001, Arlington, Virginia.

Hassan M, Amonkar M. "Aspirin Use for Primary and Secondary Prevention of Cardiovascular Disease: An Analysis of BRFSS Data." Poster presentation, 148th Annual Meeting of the American Pharmaceutical Association (APhA), March 1620, 2001, San Francisco, California.

\section{RELEVANT COURSE WORK}

Pharmacoeconomics, Econometrics, SAS, Quality of Life Assessment, Epidemiology, Data Management and Analyses, Decision Modeling, Survey Research, Qualitative Methods, Health Services Marketing, and Health Behavior Theories

\section{COMPUTER SKILLS}

Statistical and Econometric Software- SAS ${ }^{\circledR}$, SPSS $^{\circledR}$, STATA $^{\circledR}$, Ethnograph $^{\circledR}$, LIMDEP $^{\circledR}$, Decision Analysis by TreeAge ${ }^{\circledR}$

\section{HONORS and PROFESSIONAL AFFILIATIONS}

WVU Graduate Research Award ISPOR Poster Finalist Award: Seventh Annual European Congress and Eight Annual International Meeting Rho Chi National Pharmacy Honor Society American Pharmaceutical Association (APhA) (2000 - current) International Society for Pharmacoeconomics and Outcomes Research (ISPOR) (2000 - current) Institute of Occupational and Environmental Health (IOEH) Research Process Council Treasurer- WVU ISPOR Chapter Judge- Podium Presentations (ISPOR 2002) 